Variedades Inerciais Aproximadas

e Métodos de Galerkin Não Linear para as Ėquações de Água Rasa

José Waiter Cárdenas Sotil

\author{
TESE APRESENTADA \\ AO \\ INSTITUTO DE MATEMÁTICA E ESTATÍSTICA \\ DA \\ UNIVERSIDADE DE SÃO PAULO \\ PARA A OBTENCÃO DO GRAU DE DOUTOR \\ EM
}

MATEMÁTICA APLICADA

Área de Concentração: Análise Numérica

Orientador: Prof. Dr. Saulo Rabello Maciel de Barros

Durante a elaboração desta tese, o autor recebeu apoio financeiro da CAPES.

-São Paulo, Maio de 1999- 


\title{
Variedades Inerciais Aproximadas e Métodos de Galerkin Não Linear para as Equações de Águas Rasa
}

\author{
Este exemplar corresponde à redação final \\ da tese devidamente corrigida e defendida \\ por José Walter Cárdenas Sotil, \\ e aprovada pela comissão julgadora.
}

São Paulo, 22 de junho de 1999.

Banca examinadora:

- Prof. Dr. Saulo Rabello Maciel de Barros (Presidente) -IME-USP

- Prof. Dr. Sérgio Muniz Oliva Filho-IME-USP

- Prof. Dr. José Alberto Cuminato-ICMC-USP

- Prof. Dr. Marco Antonio Raupp -LNCC

- Prof. Dr. Júlio Cesar Ruiz Claeyssen -UFRGS 
Aos meus pais,

Pedro (em memória) e Yolanda: por seus ensinamentos e dedicação.

À Anneli, esposa e amiga, e aos meus filhos André e Anneli, por partilharem a minha vida.

Aos meus familiares em Perú. 


\section{Agradecimentos}

Agradeço as pessoas que participaram, direta ou indiretamente, deste trabalho.

Ao meu orientador Prof. Saulo M.R. Barros: a sua experiência na área de análise numérica foi o ponto de apoio para o sucesso deste trabalho. Meus agradecimentos e admiração por sua extrema competência e capacidade, assim como a sua amizade em todos estes anos.

Ao Prof. Marco Antonio Raupp, que prôpos a pesquisa sobre os métodos de Galerkin Não Linear, e me orientou no início de meu doutorado.

Ao Prof. Mark Thompson, pela inúmeras sugestões e críticas construtivas no desenvolvimento das estimativas para as Variedades Inerciais Aproximadas.

Ao Prof. Jorge Sotomayor, pelo respaldo inicial como orientador de programa.

Ao Prof. Sérgio Muniz Oliva, pelas suas observações e sugestões na versão final deste trabalho.

Ao pessoal do IME, professores e funcionários, especialmente ao pessoal da Pós-Graduação e da Matemática Aplicada por seu excelente trato e amabilidade.

Aos Professores e colegas da área de Análise Numérica, assim como a meus colegas do Mercosul e a meus conterrâneos do Perú, com os quais compartilhei muitos momentos agradáveis.

Aos professores do IME-UFRGS no meu mestrado, especialmente ao Prof. Júlio Ruiz Claeyssen, e aos professores da Facultad de Ciencias da Universidad Nacional de Ingeniería em Lima-Perú na minha graduação, especialmente aos Profs. Eugen Blum (em memória), Mauro Chumpitaz e Felix Escalante, pela sua contribuição na minha formação profissional. 


\begin{abstract}
In this thesis we study Approximate Inertial Manifolds for a model based on the shallowwater equations on the f-plane, which includes viscous terms and a forcing term in the vertical and employs periodic boundary conditions. We prove that the solutions of the system of equations related to the approximated inertial manifolds converge to the solutions of the original system, establishing error estimates as well. For numerical approximations we consider Galerkin type methods and propose a Non Linear Galerkin Method. We compare this scheme with classic Galerkin Methods under the aspects of computational efficiency, stability and accuracy. Our numerical scheme is based on a pseudospectral method, with a second order three time-level temporal discretization. A stability analysis, based on different linearizations, is also carried out.
\end{abstract}




\section{Resumo}

Nesta tese estudamos as Variedades Inerciais Aproximadas para um modelo derivado das equações de água rasa no chamado plano-f, considerando a inclusão de termos viscosos, uma forçante na vertical e condições de contorno periódicas. Demonstramos que as soluções do sistema associado às variedades inerciais aproximadas convergem para as soluções do sistema original, bem como estabelecemos estimativas de erro. Sob o ponto de vista numérico estudamos aproximações por métodos tipo Galerkin, propondo um método de Galerkin não linear para as equações de água rasa e comparando-o com o método de Galerkin linear quanto a eficiência computacional, estabilidade e precisão. O esquema proposto faz uso de um método pseudo-espectral com discretização temporal de segunda ordem, com três níveis no tempo. Estabelecemos ainda a estabilidade dos métodos sob dois tipos de linearização do campo das velocidades. 


\section{Sumário}

$\begin{array}{ll}\text { Introdução } & 1\end{array}$

1 Variedades Inerciais Aproximadas $\quad 6$

1.1 Formulação do modelo e preliminares . . . . . . . . . . . . . . 7

1.1.1 Espaços Funcionais . . . . . . . . . . . . . . . . . . . . 9

$1.1 .2 \quad \mathrm{O}$ operador $A_{0} \ldots \ldots \ldots \ldots 11$

1.1.3 Desigualdades de Poincaré e Sobolev . . . . . . . . . . . . . . 12

1.1.4 Conceitos de dinâmica de sistemas com dimensão infinita . . . . . . 13

1.1.5 Lemas de Gronwall . . . . . . . . . . . . . . . . . . . . . 15

1.2 Limitação das soluções . . . . . . . . . . . . . . . . . . . . . 16

1.3 As equações adimensionalizadas e estimativas a priori . . . . . . . . . . . 19

1.3.1 Existência de conjuntos absorventes em $\mathbb{H} \times H$ para a dinâmica do sistema (1.24)-(1.25). . . . . . . . . . . . . . . 20

1.3.2 Existência de conjuntos absorventes em $\mathbb{V} \times V$ e atratores em $\mathbb{H} \times H$ para a dinâmica do sistema (1.24)-(1.25) . . . . . . . . . . . . 23

1.4 Estimativas de maior ordem f. . . . . . . . . . . . . 26

1.4.1 Estimativas para $\xi$ em $\mathbb{H}_{p}^{2} \times H_{p}^{2}$, e para $\xi_{t}$ em $\mathbb{H} \times H \ldots \ldots . . . .26$

1.4.2 Estimativa para $(-\Delta)^{3 / 2} \xi$ em $\mathbb{H} \times H \ldots \ldots . \ldots . \ldots 34$

1.4.3 Estimativa para $\xi_{t}$ em $\mathbb{V} \times V \ldots \ldots \ldots \ldots$

1.5 Construção da Variedade Inercial Aproximada . . . . . . . . . . . . . . . . 40

1.5.1 Estimativas de erro para a aproximação por VIA das equações de água rasa . . . . . . . . . . . . . . . . . 46 46 
1.5.1.1 Estimativa de erro para os modos baixos . . . . . . . 46

1.5.1.2 Estimativa de erro para os modos altos . . . . . . . . . 51

1.5.2 Estimativa de erro para a aproximação por Galerkin linear . . . . . 52

2 Método numérico de Galerkin Não Linear $\quad 55$

2.1 Métodos de Galerkin para as equações de Água Rasa . . . . . . . . . . . . 71

2.1 .1 Descrição do método linear . . . . . . . . . . . . . . 72

2.1.1.1 Implementação numérica . . . . . . . . . . . . 78

2.1.1.2 Resultados numéricos . . . . . . . . . . . . 80

2.1.2 Descrição do método de Galerkin Não Linear . . . . . . . . . . . . . 85

2.1.2.1 Implementação numérica . . . . . . . . . . . . . . . 90

2.1.2.2 Resultados numéricos . . . . . . . . . . . . 92

2.2 Análise da Estabilidade . . . . . . . . . . . . . . . . . 107

2.2.1 Estabilidade: Linearização em torno de estado básico constante (três níveis no tempo) . . . . . . . . . . . . . . 109

2.2.2 Estabilidade: Linearização em torno de estado básico com dependência espacial (2 níveis no tempo) . . . . . . . . . . . . . . 112 


\section{Introdução}

Neste trabalho estudamos Variedades Inerciais Aproximadas para um modelo derivado das equações de água rasa no chamado plano-f, considerando a inclusão de termos viscosos, uma forçante na vertical e condições de contorno periódicas. Além disso, propomos e analisamos esquemas numéricos baseados no método de Galerkin não linear para estas equações, e os comparamos ao método de Galerkin clássico quanto a eficiência computacional, estabilidade e precisão.

O modelo que aqui consideramos foi empregado nos estudos de Lorenz [46], [47], que utilizou decomposições espectrais (aplicando a decomposição de Helmholtz ao campo da velocidade, e considerando só 3 modos para cada variável), obtendo um sistema de equações diferenciais ordinárias de dimensão finita. Experimentos numéricos (ver Curry e Winsand [13], Legras e Vautard [44], Kopell [41]) demonstraram uma dinâmica altamente complexa para estes modelos. Nestes trabalhos não foi estabelecido o vínculo com as equações originais através de estimativas de erro das aproximações utilizadas.

Nesta tese, estabelecemos estimativas de maior ordem (nos espaços de Sobolev $H^{2}$ e $H^{1}$ ) para a limitação das soluções e suas derivadas temporais. Para tanto temos que assumir por hipótese a limitação ( e a existência) das soluções das equações de água rasa em $L_{2}$ e empregamos a metodologia introduzida por Heywood e Rannacher [31], [32]. Com base em nossas estimativas de limitação, demonstramos a convergência das soluções do sistema associado às variedades inerciais aproximadas para as soluções do sistema original, bem como estabelecemos estimativas de erro. Também são estabelecidas estimativas de erro 
para a aproximação de soluções das equações de água rasa pelo método de Galerkin. Investigações nesta linha foram feitas para as equações incompressíveis bi-dimensonais de Navier-Stokes [16], [18], [23], [24], [25], [26], [37], [39], [45], [60], [67], [70] equação de reação-difusão [50], e [9], [28], [29], [36], [38], [49], [58], [59], [68], [71] para outras equações. Em [6], [7] iniciamos o estudo das variedades inerciais aproximadas para o modelo de Lorenz. A principal dificuldade na extensão da teoria das variedades inerciais aproximadas das equações de Navier-Stokes para o modelo de água-rasa reside no fato de que neste último o divergente do campo de velocidades não se anula e portanto as soluções não podem ser calculadas no espaço vetorial dos campos com divergente nulo. Temos que carregar a equação de conservação de massa explicitamente e estabelecer limitações para termos não lineares essencialmente diferentes dos presentes na equação de Navier-Stokes.

Sob o ponto de vista numérico estudamos aproximações por métodos tipo Galerkin, propondo um método de Galerkin não linear para as equações de água rasa e comparando-o com o método de Galerkin linear. O método proposto é baseado na decomposição das soluções em modos baixos e altos. A equação para os modos baixos é uma equação prognóstica, na qual a interação não linear entre modos altos e baixos é levada em conta, desprezando-se no entanto interações apenas entre modos altos. Já a equação dos modos altos é escrita como uma equação diagnóstica (pode também ser posta na forma prognóstica), em que apenas a interação não linear entre modos baixos é considerada. Desta forma, os modos altos são determinados diagnosticamente a partir dos modos baixos, de maneira similar ao que é feito no estudo das variedades inerciais aproximadas.

A implementação proposta faz uso de um método pseudo-espectral com discretização temporal de segunda ordem, com três níveis no tempo. As malhas utilizadas no tratamento pseudo-espectral são escolhidas de maneira a garantir que os produtos (termos quadráticos) entre funções de malha sejam calculados livres de 'aliasing', ou seja, as projeções destes produtos nos subespaços de dimensão finita empregados nos métodos de Galerkin são avaliadas sem erros devidos à coincidência de diferentes modos restritos às malhas. Para um mesmo truncamento $\mathbf{N}$ o método de Galerkin Linear requer malhas mais finas que o não linear. No total, o método de Galerkin não linear é cerca de $12 \%$ menos 
custoso que o de Galerkin linear. Consideramos ainda o congelamento dos modos altos por um número fixo de iterações, reduzindo o custo computacional do método não linear a menos da metade do custo de Galerkin linear. Experimentos numéricos mostram que estes congelamentos dos modos altos podem ser feitos sem grande impacto na precisão (o que está de acordo com a teoria das Variedades Inerciais Aproximadas para um N suficientemente grande). Em geral, a precisão do método não linear com um determinado número de modos é quase tão boa quanto a do correspondente método de Galerkin linear, porém a um custo computacional menor. Este comportamento dos métodos de Galerkin linear e não-linear foi primeiramente investigado através da equação de Burgers unidimensional, com termo viscoso e sem forçante, caso em que soluções analíticas conhecidas foram usadas para se aferir a precisão.

Apresentamos ainda uma análise da estabilidade dos métodos de Galerkin. Primeiramente, usando estimativas de energia e uma linearização em torno de um estado básico constante, derivamos condições suficientes para a estabilidade dos esquemas. As condições para o método não-linear coincidem com as condições para Galerkin linear com metade dos modos (devendo-se este fato à equação diagnóstica dos modos altos e à linearidade das equações resultantes). Analisamos ainda a estabilidade empregando uma linearização em torno de um estado básico com variação espacial. Neste caso há interação entre os modos altos e baixos e com uma hipótese extra, de que o divergente do campo de velocidades do estado base seja suficientemente pequeno, estabelecemos a estabilidade dos esquemas. Novamente, as condições de estabilidade do método não linear são menos restritivas que as do método linear com mesmo número de modos.

Comparações entre métodos de Galerkin linear e não linear para outras equações, referentes ao regime permanente, foram consideradas por diversos autores. Quando as soluções das equações envolvidas são incompatíveis com as funções de base no contorno, o método de Galerkin não linear tem a vantagem de diminuir a intensidade do fenômeno de Gibbs (Heywood [32]). Quando as soluções apresentam menos regularidade é mais interessante seu uso do que no caso de soluções mais suaves, de acordo com Jones, Margolin e Titi 
[40] (que fizeram simulações numéricas da equação de Burgers-1D com condições de contorno de Dirichlet, e da equação de Kuramoto-Shivashinsky com condições de contorno periódicas). Quanto à eficiência computacional Jabertau, Rosier e Temam [33], [34] aplicam o método de Galerkin não linear para as equações incompressíveis de Navier-Stokes em duas dimensões. Seus experimentos indicam um ganho de $30 \%$ a $40 \%$ na eficiência deste método (com os modos altos congelados um número determinado de iterações), em relação ao método de Galerkin linear.

Neste trabalho atingimos um ganho superior a $50 \%$ em eficiência no caso das equações de água rasa, quando o congelamento dos modos é empregado. Quanto à precisão relativa dos métodos de Galerkin linear e não-linear preferimos a visão de que o método de Galerkin não linear (em sua visão prática, em que os modos altos são truncados em $2 N$, e não vistos como o espaço de dimensão infinita complementar aos modos baixos) é capaz de práticamente oferecer a mesma precisão que o método de Galerkin linear de mesma resolução a um custo significativamente menor. Foi o que observamos, mesmo utilizando soluções bastante regulares nas equações de água rasa.

Apresentamos a seguir a estrutura da tese.

As variedades inerciais aproximadas para o modelo de água rasas são estudadas no capítulo 1. Na seção 1.1 o modelo em estudo é formulado e são definidos os espaços funcionais, operadores e desigualdades necessárias para o estudo das variedades inerciais aproximadas. Na seção 1.2 é estabelecida a limitação das soluções das equações de água rasa sob uma hipótese restritiva tomada de Agoshkov [1] (ver Hipótese 1.1 e a Estimativa 1.1). No caso geral assumimos a existência e a limitação das soluções num intervalo $[0, T]$ (as quais são ainda questões em aberto). Na seção (1.3), as equações são adimensionalizadas, sendo estabelecidas estimativas para as soluções a prazo longo na norma $L_{2}$ e $H^{1}$ (ver Estimativas 1.2 e 1.4). Seguindo o método de Heywood e Rannacher [32], na seção 1.4 são estabelecidas estimativas de maior ordem para as soluções e suas derivadas no tempo (ver Lemas 1.1, 1.2 e 1.3). Na seção 1.5 são definidas as variedades inerciais para as equações de água rasa (ver o sistema 1.60 e a equação 1.61), e são estabelecidas estimativas de erro para os modos baixos e os modos altos no método de Galerkin não linear, as quais são 
da ordem $\lambda_{N}^{-3 / 2}$, onde $\lambda_{N}$ é o $N$-ésimo autovalor do operador $-\Delta$ (ver Proposição (1.2), o Lema 1.6 e o Teorema 1.2). Também são estabelecidas estimativas de erro para o método de Galerkin linear, as quais são da ordem $\lambda_{N}^{-1}$ (ver Proposição 1.3).

No capítulo 2 é feita a implementação dos métodos de Galerkin linear e não linear para as equações de água rasa. Previamente, para testar o comportamento destes métodos, usamos as equações de Burgers com condições de contorno periódicas em uma dimensão, as quais tem solução conhecida (ver equações 2.5 e 2.6). Os resultados numéricos da aplicação do método de Galerkin linear (ver a equação 2.7) e do método de Galerkin não linear (ver as equações 2.8 e 2.9), são apresentados nas figuras 2.1-2.15 (aspectos referentes à precisão) e nas tabelas 2.1-2.3 (resultados sobre a estabilidade).

Na seção 2.1 são estudados os métodos de Galerkin linear e não linear para as equações de água rasa. Nossa proposta para a implementação do método de Galerkin linear e sua solução é apresentada em detalhe na seção 2.1.1, e o resultado desta discretização é o sistema 2.28-2.30. Para analisar o custo computacional deste método, a implementação numérica é resumida na seção 2.1.1.1. As condições iniciais para uma simulação numérica são geradas na seção 2.1.1.2 e seus resultados são apresentados na figuras 2.16-2.18.

Na seção 2.1.2 apresentamos duas propostas para a implementação do método de Galerkin não linear, uma diagnóstica e outra prognóstica para as equações dos modos altos, esta discretização é resumida no sistema 2.69-2.74. A implementação dos termos não lineares é discutida e sua implementação é resumida na seção 2.1.2.1, sendo comparados o custo computacional entre estes métodos de Galerkin. Na seção 2.1.2.2 são apresentadas várias simulações comparando a eficiência entre estes métodos (ver figuras 2.21-2.32). Na figura 2.19 são mostrados os tempos de CPU deste métodos.

A estabilidade dos métodos de Galerkin para as equações de água rasa é estudada na seção 2.2. Na proposição 2.1 é demonstrada a estabilidade de Galerkin linear quando o sistema é linearizado ao redor de um campo de velocidades constante. Para um sistema com dois níveis no tempo, linearizados ao redor de um campo de velocidades independente do tempo e divergente pequeno (ver Hipótese 2.1) a estabilidade é estabelecida nas Proposições 2.2 e 2.3 para o método de Galerkin linear e não linear, respectivamente. 


\section{Capítulo 1}

\section{Variedades Inerciais Aproximadas}

No estudo das equações de Navier-Stokes incompressíveis, Foias, Manley e Temam [24] propuseram o conceito de variedade inercial aproximada, como sendo uma variedade diferencial não linear de dimensão finita que atrai exponencialmente todas as órbitas do sistema em uma sua vizinhança, que em particular contém o atrator. A existência destas variedades tém motivado o desenvolvimento de novos métodos numéricos, chamados de métodos de Galerkin não linear, para as equações de Navier-Stokes e outras equações de evolução dissipativas, tais como a equação de Kuramoto-Shivashinsky, Burgers em uma dimensão, e equações de reação-difusão. Aqui estudamos estas variedades para um modelo derivado das equações de água rasa. Na prática o método de construção das variedades baseia-se na decomposição do espaço de Hilbert das soluções. As funções base (autofunções de um operador) são divididas em dois conjuntos (modos baixos e altos) e as equações projetadas sobre estes conjuntos, separando-se as equações para os modos baixos e altos. A equação para os modos altos é considerada diagnóstica, com interação somente entre os modos baixos nos termos não lineares, assim os modos altos podem se expressar como função de um número finito de modos (a variedade inercial aproximada). Colocando esta variedade na equação dos modos baixos, temos um sistema em dimensão finita que pode-se resolver para os modos baixos. A seguir apresentamos o modelo de água rasa e algumas definições e resultados básicos sobre os espaços funcionais necessários. $\mathrm{Na}$ seção 1.5 esclarecemos a teoria das variedades inerciais aproximadas, construindo estas para nosso modelo de águas rasas. Nosso objetivo principal neste capítulo é estabelecer 
estimativas de erro para os métodos de Galerkin linear e não linear para nosso modelo.

\subsection{Formulação do modelo e preliminares}

Em um artigo publicado em 1980 [46], Lorenz introduziu um modelo derivado das equações de água rasa sobre o chamado plano $f$, incluindo um termo forçante de massa $F(r)$ independente do tempo. Observamos que toda a massa adicionada ao sistema é suposta movimentando-se a uma velocidade localmente apropriada. O fluido (incompressível) se movimenta sobre uma superfície com topografia variável $\tilde{h}(r)$. A velocidade horizontal (denotada por $\vec{V}(r, t))$ é suposta constante sobre a altura do fluido. A superfície superior do fluido é dado por $z(r, t)+h$, onde $z$ representa a variação da altura do fluido com respeito a seu valor médio $h$. As velocidades verticais do fluido são determinadas pela continuidade da massa. Os movimentos verticais e horizontais são amortecidos difusivamente por coeficientes $\nu$ e $\kappa$, onde $\nu \sim 2.25 \times 10^{6} \mathrm{~m}^{2} / \mathrm{s}$ é tomado por Lorenz como sendo a viscosidade turbulenta (Ilyin usou um valor de $\nu \sim 1.4 \times 10^{6} \mathrm{~m}^{2} / \mathrm{s}$ num modelo global). Na Figura 1.1 se representa o modelo descrito acima.

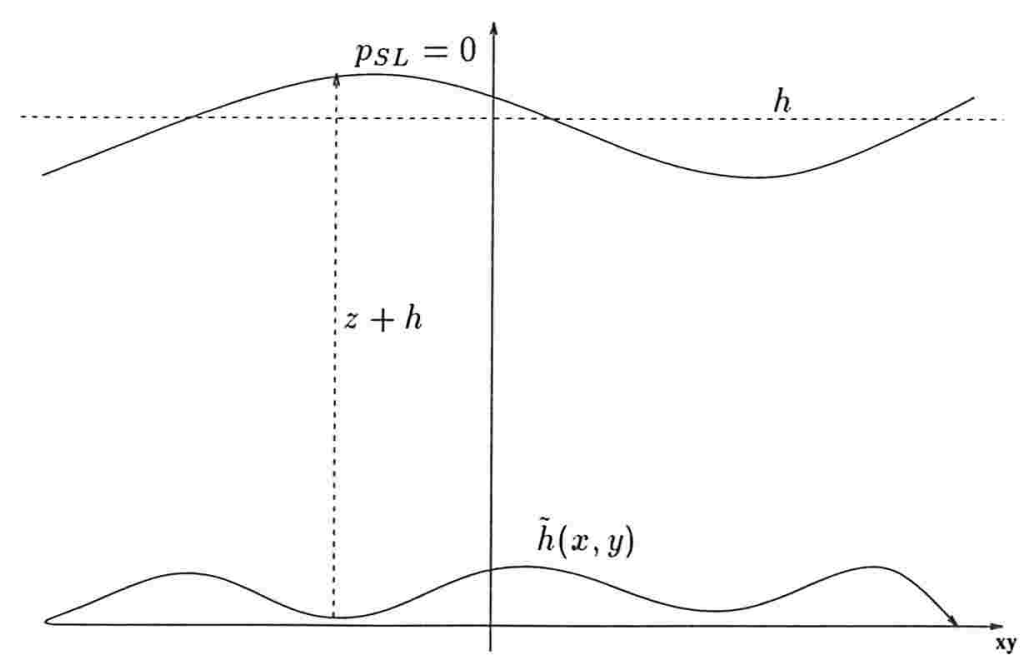

Figura 1.1: Aproximação de Água Rasa

onde: $h=$ altura média da superfície livre, $z(x, y, t)=$ desvio do fluido com respeito a $h$, $\tilde{h}(x, y)=$ topografia da terra, $p_{S L}=$ pressão na superfície livre. 
As equações de água rasa sobre o plano $f$ em coordenadas locais, serão derivadas a seguir, a partir das equações de Euler (incluindo a força de Coriolis) incompressíveis :

$$
\begin{aligned}
\frac{\partial}{\partial t} \vec{V}+(\vec{V} \cdot \nabla) \vec{V} & =-\frac{1}{\rho} \nabla p-f \vec{k} \times \vec{V} \\
\nabla \cdot \vec{V}+w_{z} & =0
\end{aligned}
$$

onde $\vec{k}=(0,0,1)$ é o vetor unitario na vertical, $\vec{V}$ é a velocidade horizontal, $w$ é a velocidade vertical, $p$ é a pressão, $\rho$ é a densidade do fluido e $f$ é o parâmetro de Coriolis, suposto constante. A equação (1.1) representa a equação de movimento, e a equação (1.2) representa a equação de continuidade.

Com a hipótese de termos um fluido homogêneo incompressível, a atmosfera se desenvolve até uma altura média $h \sim 8 k m$, e uma superfície livre é desenvolvida. Considerando uma escala horizontal da ordem de $10^{3} \mathrm{~km}$, a hipótese de equilíbrio hidrostático é satisfeita:

$$
\frac{d p}{d z}=-\rho g
$$

Integrando: $\int_{p_{S L}=0}^{p} d \tilde{p}=-\int_{z+h}^{z_{1}} \rho g d \tilde{z}$ (onde $z_{1}$ é constante), resulta $p=\rho g\left(z+h-z_{1}\right)$, e portanto:

$$
\frac{1}{\rho} \nabla p=g \nabla z
$$

Por outro lado, integrando a equação de continuidade (1.2) temos:

$w_{S L}-w_{\tilde{h}}=\int_{w_{\tilde{h}}}^{w_{S L}} d w=-\int_{\tilde{h}}^{z+h}(\nabla \cdot \vec{V}) d \tilde{z}=-(\nabla \cdot \vec{V})(z+h-\tilde{h}) \quad($ pois $\vec{V}$ independe de $z)$

Sendo $h+z$ e $\tilde{h}$ superfícies materiais, obtemos:

$$
w_{S L}-w_{\tilde{h}}=\frac{d}{d t}(h+z)-\frac{d \tilde{h}}{d t}=-(\nabla \cdot \vec{V})(z+h-\tilde{h})
$$

Substituindo (1.4) em (1.1) e considerando (1.5) temos as Equações de Água-Rasa :

$$
\begin{aligned}
\frac{d \vec{V}}{d t} & =-g \nabla z-f \vec{k} \times \vec{V} \\
\frac{d}{d t}(z-\tilde{h}) & =-(h+z-\tilde{h})(\nabla \cdot \vec{V})
\end{aligned}
$$


Agora, como em [46], [47] incluímos a hipótese de que os movimentos horizontais e verticais do fluido são difusivamente amortecidos com coeficientes de amortecimento $\nu$ e $\kappa$. O sistema ainda pode incluir um termo forçante $F$ que independe do tempo, com a introdução ou retirada de massa do fluido. Esta situação é similar a forçar térmicamente o fluido [13]. Toda a massa adicionada ao sistema é suposta movimentando-se a uma velocidade localmente apropriada. Com estas considerações, as equações (1.6) nas variáveis dependentes $\vec{V}$ e $z$ tomam a forma:

$$
\begin{aligned}
\frac{\partial \vec{V}}{\partial t}+\vec{V} \cdot \nabla \vec{V}+f \vec{k} \times \vec{V}+g \nabla z & =\nu \Delta \vec{V} \\
\frac{\partial z}{\partial t}+\nabla \cdot(h+z-\tilde{h}) \vec{V} & =\kappa \Delta z+F
\end{aligned}
$$

onde $f, g, \nu, \kappa, h, \tilde{h}$, e $F$ são assumidos conhecidos.

No que segue, a topografia inferior não é incluída, isto é $\tilde{h}=0$, e consideramos as equações (1.7) e (1.8) definidas numa região plana $\mathcal{Q}=[0,2 \pi L] \times[0,2 \pi L]$ com condições de contorno periódicas. Definimos $\vec{V}, z, F$, e os operadores $\nabla, \Delta$ nas variáveis independentes $(x, y) \in \mathcal{Q}$. Em [6], [46] e [47], encontra-se uma dedução mais detalhada destas equações .

\subsubsection{Espaços Funcionais}

Denotamos por $L_{2}(\Omega)$ o espaço das funções com valores reais sobre $\Omega$, as quais são quadrado integráveis para a medida de Lebesgue $d \bar{x}=d x d y$ com o produto interno e a norma:

$$
(u, v)=\int_{\Omega} u(x, y) v(x, y) d x d y, \quad|u|=\{(u, u)\}^{1 / 2},
$$

e por $H^{m}, m \in \mathbb{N}$, o espaço de Sobolev das funções em $L_{2}(\Omega)$, junto com suas derivadas de ordem $\leq m$. Este é um espaço de Hilbert para o produto interno e a norma:

$$
(u, v)_{m}=\sum_{[\alpha] \leq m}\left(D^{\alpha} u, D^{\alpha} v\right), \quad|u|_{m}=\left\{(u, u)_{m}\right\}^{1 / 2},
$$

onde, $\alpha=\left(\alpha_{1}, \alpha_{2}\right), \alpha \in \mathbb{N}^{2} ;[\alpha]=\alpha_{1}+\alpha_{2}$, e $D^{\alpha}=\partial^{\alpha_{1}+\alpha_{2}} /\left(\partial x_{1}^{\alpha_{1}} \partial x_{2}^{\alpha_{2}}\right)$. 
Seja $H_{p}^{m}(\mathcal{Q}), m \in \mathbb{N}$. o espaço de funções em $H_{l o c}^{m}\left(\mathbb{R}^{2}\right.$ ) (isto é: $u / \mathcal{O} \in H^{m}(\mathcal{O})$ para cada conjunto aberto limitado $\mathcal{O}$ ) as quais são periódicas com domínio $\mathcal{Q}=[0,2 \pi L] \times[0,2 \pi L]$ :

$$
u(x+2 \pi L, y)=u(x, y), \quad u(x, y+2 \pi L)=u(x, y) .
$$

Se $m=0, H_{p}^{0}(\mathcal{Q})$ coincide com $L_{2}(\mathcal{Q})$. Para $m \in \mathbb{N}, H_{p}^{m}(\mathcal{Q})$ é um espaço de Hilbert com o produto interno e a norma, por ele induzida:

$$
(u, v)_{m}=\sum_{0 \leq[\alpha] \leq m} \int_{\mathcal{Q}} D^{\alpha} u(x) D^{\alpha} v(x) d x, \quad|u|_{m}=\{(u, u)\}^{1 / 2}
$$

As funções em $H_{p}^{m}(\mathcal{Q})$ são facilmente caracterizadas por suas expansões em séries de Fourier,

$$
H_{p}^{m}(\mathcal{Q})=\left\{u, u(x)=\sum_{\mathbf{k} \in \mathbb{Z}^{2}} c_{\mathrm{k}} e^{i \mathrm{k} \cdot x / L}, \quad \bar{c}_{\mathrm{k}}=c_{-\mathrm{k}}, \quad \sum_{\mathbf{k} \in \mathbb{Z}^{2}}|\mathrm{k}|^{2 m}\left|c_{\mathbf{k}}\right|^{2}<\infty\right\}
$$

Também denotamos,

$$
\dot{H}_{p}^{m}(\mathcal{Q})=\left\{u \in H_{p}^{m}(\mathcal{Q}): c_{0}=\frac{1}{4 \pi^{2} L^{2}} \int_{\mathcal{Q}} u=0\right\}
$$

Estendemos a definição de $H_{p}^{m}(\mathcal{Q})$ para $m \in \mathbb{R}, m \geq 0$ por (1.9), o qual é um espaço de Hilbert para a norma $\left\{\sum_{\mathrm{k} \in \mathbb{Z}^{2}}\left(\left(1+|\mathrm{k}|^{2 m}\right)\left|c_{\mathrm{k}}\right|^{2}\right\}^{1 / 2}\right.$, equivalente à norma $|u|_{m}$. Para $m \in \mathbb{R}$, definimos $\dot{H}_{p}^{m}(\mathcal{Q})$ por (1.10), o qual é um espaço de Hilbert para a norma $\left\{\sum_{\mathbf{k} \in Z^{2}}|\mathrm{k}|^{2 m}\left|c_{\mathbf{k}}\right|^{2}\right\}^{1 / 2}$.

Os espaços funcionais para $\vec{V}$ e $z$.-

A velocidade horizontal do fluido $\vec{V}=\left(\begin{array}{c}v_{1} \\ v_{2}\end{array}\right)$ é definida no espaço produto $\dot{\mathbb{H}}_{p}^{1}(\mathcal{Q})=$ $\dot{H}_{p}^{1}(\mathcal{Q}) \times \dot{H}_{p}^{1}(\mathcal{Q})$, e a altura $z$ é definida em $\dot{H}_{p}^{1}(\mathcal{Q})$.

Freqüentemente usaremos as normas $|$.$| em L_{2}(\mathcal{Q}):|\vec{V}|=\left\{\left|v_{1}\right|^{2}+\left|v_{2}\right|^{2}\right\}^{1 / 2},|z|$; e as normas $\|\cdot\|=\{(., .)\}^{1 / 2}$ em $\dot{H}_{p}^{1}(\mathcal{Q}):\|\vec{V}\|=\left\{\left\|v_{1}\right\|^{2}+\left\|v_{2}\right\|^{2}\right\}^{1 / 2},\|z\|$. 


\subsubsection{O operador $A_{0}$}

Com os espaços: $H=\dot{H}_{p}^{0}(\mathcal{Q})$ e $V=\dot{H}_{p}^{1}(\mathcal{Q})$, consideramos o problema:

$$
\text { Dado } f \in H \text {, achar } u \in V \text { tal que }-\Delta u=f \text {. }
$$

Tomando as séries de Fourier: $u=\sum_{\mathrm{k} \in \mathbb{Z}^{2}} \hat{u}_{k} e^{i \mathrm{k} \cdot x / L}, \quad f=\sum_{\mathrm{k} \in \mathbb{Z}^{2}} \hat{f}_{k} e^{i \mathrm{k} \cdot x / L}$, e substituindo em (P) obtemos:

$$
\hat{u}_{\mathrm{k}}=\frac{\hat{f}_{\mathrm{k}} L^{2}}{|\mathrm{k}|^{2}} ; \quad \mathrm{k} \in \mathbb{Z}^{2}, \quad \mathrm{k} \neq 0 .
$$

Fica assim definida uma aplicação $f \longrightarrow u$ de $H$ sobre $D\left(A_{0}\right)=\{u \in H, \quad-\Delta u \in H\}=$ $\dot{H}_{p}^{2}(\mathcal{Q}) \cap H=\dot{H}_{p}^{2}(\mathcal{Q})$, e a aplicação inversa :

$A_{0}: \dot{H}_{p}^{2}(\mathcal{Q}) \longrightarrow H ; \quad A_{0} u=-\Delta u, \quad \forall u \in D\left(A_{0}\right)$ é bem definida.

O operador $A_{0}$ é um operador linear, positivo, não-limitado e auto-adjunto sobre $H$, e pode-se definir as potências de $A_{0}^{\alpha}, \quad \alpha \in \mathbb{R}$, com domínio $D\left(A_{0}^{\alpha}\right)$ em $H$ (ver [66]).

\section{Autofunções de $A_{0 .-}$}

O operador $A_{0}^{-1}$ é linear, contínuo, de $H$ em $D\left(A_{0}\right)$, e posto que a injeção de $D\left(A_{0}\right)$ em $H$ é compacta, $A_{0}^{-1}$ pode-se considerar como um operador compacto em $\mathrm{H}$ (ver [65], [66]). Sendo $A_{0}$ auto-adjunto, este possui uma seqüência de autofunções $v_{j}, j \in \mathbb{N}$, formando uma base ortonormal de $H$,

$$
\begin{aligned}
& A_{0} \mathrm{v}_{j}=\lambda_{j} \mathrm{v}_{j}, \quad \mathrm{v}_{j} \in D\left(A_{0}\right), \\
& 0<\lambda_{1} \leq \lambda_{2} \cdots, \quad \lambda_{j} \rightarrow \infty \text { para } j \rightarrow \infty
\end{aligned}
$$

Explicitamente os autovalores e autofuncões de $A_{0}$ são dados por:

$$
\begin{array}{ll}
\lambda_{\mathrm{k}}=\frac{1}{L^{2}}|\mathrm{k}|^{2}, \\
\mathrm{v}_{\mathrm{k}}=\frac{1}{\sqrt{\pi L}} \cos \left(\frac{\mathrm{k} \cdot x}{L}\right), \quad \tilde{\mathrm{v}}_{\mathrm{k}}=\frac{1}{\sqrt{\pi L}} \sin \left(\frac{\mathrm{k} \cdot x}{L}\right) .
\end{array}
$$

O operador $A_{0}$ pode ser definido no espaço produto por:

$$
A_{0} \vec{V}=-\Delta \vec{V}=-\Delta\left(\begin{array}{l}
v_{1} \\
v_{2}
\end{array}\right)=\left(\begin{array}{c}
-\Delta v_{1} \\
-\Delta v_{2}
\end{array}\right), \quad \forall \vec{V} \in \dot{H}_{p}^{2}(\mathcal{Q})=\dot{H}_{p}^{2}(\mathcal{Q}) \times \dot{H}_{p}^{2}(\mathcal{Q})
$$

Todas as propriedades estudadas de $A_{0}$ são estendidas para o espaço produto. 


\subsubsection{Desigualdades de Poincaré e Sobolev}

As seguintes desigualdades serão freqüentemente usadas:

D1.- Desigualdade de Poincaré (ver por exemplo [66]):

$$
\begin{aligned}
& \left\{\begin{array}{c}
|\vec{V}| \\
|z|
\end{array} \leq c_{0}\left\{\begin{array}{c}
\|\vec{V}\| \\
\|z\|
\end{array} \quad \forall \vec{V} \in \mathbb{V}=V \times V, \quad \forall z \in V\right.\right. \\
& \text { onde } c_{0}=\lambda_{1}^{-1}=L^{2} / 2
\end{aligned}
$$

D2. $\left\{\begin{array}{l}|\vec{V}|_{L_{4}(\mathcal{Q})} \\ |z|_{L_{4}(\mathcal{Q})}\end{array} \leq 2\left\{\begin{array}{c}|\vec{V}|^{1 / 2}\|\vec{V}\|^{1 / 2} \\ |z|^{1 / 2}\|z\|^{1 / 2}\end{array} \quad \forall \vec{V} \in \mathbb{V}, \quad \forall z \in V\right.\right.$.

D3.- $|\vec{V}|_{L_{\infty}(\mathcal{Q})} \leq d_{1}\left\{\begin{array}{l}|\vec{V}|^{1 / 2}|\Delta \vec{V}|^{1 / 2} \\ \|\vec{V}\|^{3 / 4}|\Delta \vec{V}|^{1 / 4}\end{array}, \quad \forall \vec{V} \in \dot{\mathbb{H}}_{p}^{2}\right.$,

$$
|z|_{L_{\infty}(\mathcal{Q})} \leq d_{1}\left\{\begin{array}{c}
|z|^{1 / 2}|\Delta z|^{1 / 2} \\
\|z\|^{3 / 4}|\Delta z|^{1 / 4}
\end{array}, \quad \forall z \in \dot{H}_{p}^{2}\right.
$$

As seguintes formas bilineares são definidas:

$$
B(\vec{U}, \vec{V})=(\vec{U} \cdot \nabla) \vec{V}, \quad B_{1}(z, \vec{V})=z \nabla \cdot \vec{V} \quad B_{2}(\vec{V}, z),=(\vec{V} \cdot \nabla) z .
$$

Para $\vec{U}, \vec{V}, \vec{W} \in \mathbb{L}_{1}(\mathcal{Q}), \quad z_{i} \in L_{1}(\mathcal{Q})$, definimos as formas trilineares:

$$
\begin{aligned}
b(\vec{U}, \vec{V}, \vec{W}) & =(B(\vec{U}, \vec{V}), \vec{W})=((\vec{U} \cdot \nabla) \vec{V}, \vec{W})=\sum_{i, j=1}^{2} \int_{\mathcal{Q}} u_{i}\left(D_{i} v_{j}\right) w_{j} d x \\
b_{1}\left(z_{1}, \vec{V}, z_{2}\right) & =\left(B_{1}\left(z_{1}, \vec{V}\right), z_{2}\right)=\left(z_{1} \nabla \cdot \vec{V}, z_{2}\right)=\sum_{i=1}^{2} \int_{\mathcal{Q}} z_{1}\left(D_{i} v_{i}\right) z_{2} d x \\
b_{2}\left(\vec{V}, z_{1}, z_{2}\right) & =\left(B_{2}\left(\vec{V}, z_{1}\right), z_{2}\right)=\left(\vec{V} \cdot \nabla z_{1}, z_{2}\right)=\sum_{i=1}^{2} \int_{\mathcal{Q}} v_{i}\left(D_{i} z_{1}\right) z_{2} d x,
\end{aligned}
$$

as quais são continuas em $\mathbb{H} \times \mathbb{V} \times \mathbb{H}, \quad H \times \mathbb{V} \times H, \quad \mathbb{H} \times V \times H$ respectivamente.

As seguintes estimativas são verificadas:

E1.- $|b(\vec{U}, \vec{V}, \vec{W})| \leq c_{1}|\vec{U}|^{1 / 2}\|\vec{U}\|^{1 / 2}\|\vec{V}\||\vec{W}|^{1 / 2}\|\vec{W}\|^{1 / 2}, \quad \forall \vec{U}, \vec{V}, \vec{W} \in \mathbb{V}$

E2.- $|b(\vec{U}, \vec{V}, \vec{W})| \leq c_{2}|\vec{U}|^{1 / 2}|\Delta \vec{U}|^{1 / 2}\|\vec{V}\||\vec{W}|, \quad \forall \vec{U} \in \dot{\mathbb{H}}_{p}^{2}, \quad \vec{V} \in \mathbb{V}, \quad \vec{W} \in \mathbb{H}$ 
E3.- $|b(\vec{U}, \vec{V}, \vec{W})| \leq c_{3}|\vec{U}|^{1 / 2}\|\vec{U}\|^{1 / 2}\|\vec{V}\|^{1 / 2}|\Delta \vec{V}|^{1 / 2}|\vec{W}|, \quad \forall \vec{U} \in \mathbb{V}, \quad \vec{V} \in \dot{\mathbb{H}}_{p}^{2}, \quad \vec{W} \in \mathbb{H}$ E4.- $\left|A_{0}^{1 / 2} B(\vec{U}, \vec{V})\right| \leq \frac{c_{4}}{\sqrt{\lambda_{1}}}\left|A_{0} \vec{U}\right|\left|A_{0} \vec{V}\right|, \quad \forall \vec{U}, \vec{V} \in D\left(A_{0}\right)$.

E5.- $\left|A_{0}^{s / 2} B(\vec{U}, \vec{V})\right| \leq \frac{c_{5}}{\lambda_{1}^{s / 2-1 / 2}}\left|A_{0}^{s / 2} \vec{U}\right|\left|A_{0}^{s+1 / 2} \vec{V}\right|, \quad s>1$

\section{Prova.-}

A prova destas desigualdades é baseada nas desigualdades D1-D3:

$|b(\vec{U}, \vec{V}, \vec{W})| \leq \sum_{i, j=1}^{2}\left|u_{i}\right|_{L_{4}}\left|D_{i} v_{j}\right|_{L_{2}}\left|w_{j}\right|_{L_{4}} \leq 4|\vec{U}|_{L_{4}}\|\vec{V}\||\vec{W}|_{L_{4}} \leq E_{1} \quad$ (por D2) $|b(\vec{U}, \vec{V}, \vec{W})| \leq \sum_{i, j=1}^{2}\left|u_{i}\right|_{L_{\infty}}\left|D_{i} v_{j}\right|_{L_{2}}\left|w_{j}\right|_{L_{2}} \leq 4|\vec{U}|_{L_{\infty}}\|\vec{V}\||\vec{W}| \leq E_{2} \quad$ (por D3)

$|b(\vec{U}, \vec{V}, \vec{W})| \leq \sum_{i, j=1}^{2}\left|u_{i}\right|_{L_{4}}\left|D_{i} v_{j}\right|_{L_{4}}\left|w_{j}\right|_{L_{2}} \leq 4|\vec{U}|_{L_{4}}\|\vec{V}\|_{L_{4}}|\vec{W}| \leq E_{3} \quad$ (por D2)

As desigualdades E1-E3 também são válidas se substituimos $b(\vec{U}, \vec{V}, \vec{W})$ por $b_{1}\left(z_{1}, \vec{V} . z_{2}\right)$ ou por $b\left(\vec{V}, z_{1}, z_{2}\right)$. A prova de E4 e E5 é feita em [11], e também são válidas para $B_{1}(z, \vec{V})$ e $B_{2}(\vec{V}, z)$.

\subsubsection{Conceitos de dinâmica de sistemas com dimensão infinita}

Seguindo [60] e [69] definamos os operadores: $S(t): u_{0} \rightarrow u(t), \quad t>0$, de $H$ em $H$, que gozam das propriedades de semigrupo:

$$
\begin{aligned}
S(t+s) & =S(t) \circ S(s), \quad s, t \geq 0 \\
S(0) & =I
\end{aligned}
$$

contínuos de $H$ em $H$. S $(t)$ descreve a dinâmica do fluido, já que liga o estado inicial $u_{0}$ com os estados futuros possíveis, $u(t), t>0$. Aos conjuntos

$$
\bigcup_{t \geq 0} S(t) u_{0} \quad \text { e } \bigcup_{t \leq 0} S(t) u_{0}
$$

chamaremos de órbitas começando e terminando em $u_{0}$.

Um estado de equilíbrio (ou estacionário) do sistema é um $u_{0} \in H$ tal que $S(t) u_{0}=u_{0}$, $\forall t>0$. $X \subset H$ é positivamente invariante para a dinâmica $S(t)$ se $S(t) X \subset X, \forall t \geq 0$, e negativamente invariante se $S(t) X \supset X, \forall t>0$. E $X$ é invariante para $S(t)$ quando 
for positivamente e negativamente invariante, isto é, $S(t) X=X, \forall t \geq 0$.

Um atrator para a dinâmica $S(t)$ é um conjunto $\mathcal{A} \subset H$ tal que

(i) $\mathcal{A}$ é invariante para $S(t)$;

(ii) $\mathcal{A}$ possui uma vizinhança aberta $\mathcal{U}$ tal que para qualquer $u_{0} \in \mathcal{U}, S(t) u_{0}$ converge para $\mathcal{A}$ quando $t \uparrow \infty$, isto é, $\lim _{t \uparrow \infty} \operatorname{dist}\left(S(t) u_{0}, \mathcal{A}\right)=0$.

O maior aberto que satisfaz (ii) é a bacia de atração de $\mathcal{A}$.

Dizemos que $\mathcal{A} \subset H$ é um atrator universal ou global para a dinâmica $S(t), t \geq 0$, se $\mathcal{A}$ é um atrator compacto que atrai os conjuntos limitados de $H$ (ver [66]).

Se $\mathcal{L}$ é um subconjunto de $H$ e $\mathcal{U}$ é um aberto contendo $\mathcal{L}$. dizemos que $\mathcal{L}$ é absorvente em $\mathcal{U}$ se as órbitas de qualquer subconjunto limitado $\mathcal{L}_{0}$ de $\mathcal{U}$ entram em $\mathcal{L}$ após um certo tempo, isto é: para qualquer $\mathcal{L}_{0} \subset \mathcal{U}, \mathcal{L}_{0}$ limitado, existe $t_{1}\left(\mathcal{L}_{0}\right)$ tal que $S(t) \mathcal{L}_{0} \subset \mathcal{L}$ para $t \geq t_{1}\left(\mathcal{L}_{0}\right)$

Dados $u_{0} \in H$ ou $\mathcal{A} \subset H$, definimos os conjuntos $w$-limite

$$
\begin{aligned}
& w\left(u_{0}\right)=\bigcap_{s \geq 0} \overline{\bigcup_{t \geq s} S(t) u_{0}}, \\
& w(\mathcal{A})=\bigcup_{s \geq 0} \overline{\bigcap_{t \geq s} S(t) \mathcal{A}},
\end{aligned}
$$

onde os fechos são tomados em $H$. É fácil ver que $\varphi \in w(\mathcal{A})$ se e somente se existirem seqüências de elementos $\varphi_{n} \in \mathcal{A}$ e $t_{n} \rightarrow \infty$ tais que $S\left(t_{n}\right) \varphi_{n} \rightarrow \varphi$ quando $n \rightarrow \infty$.

É fácil ver que a existência de um atrator universal $\mathcal{A}$ para uma dinâmica $S(t)$ implica a existência de um conjunto absorvente. Inversamente em, [64], [66] demonstra-se que uma dinâmica $S(t)$ que possua conjunto absorvente e goze de algumas outras propriedades possui também um atrator universal. Tal informação é sintetizada no seguinte

Teorema 1.1 Na hipótese mais geral de $H$ ser um espaço métrico, supomos dada uma família de operadores $S(t), t>0$, satisfazendo (1.11) e (1.12), contínuos de $H$ em $H$, e uniformemente compactos para $t$ grande, isto é,

$$
\left\{\begin{array}{c}
\forall \text { limitado } \mathcal{L}, \text { existe } t=t_{0}(\mathcal{L}) \text { tal que } \\
\bigcup_{t \geq t_{0}} S(t) \mathcal{L} \text { é relativamente compacto em } H .
\end{array}\right.
$$


Supomos também que existe um aberto $\mathcal{U}$ e um subconjunto limitado $\mathcal{L}$ de $\mathcal{U}$ tal que $\mathcal{L}$ é absorvente em $\mathcal{U}$.

Então o conjunto w-limite de $\mathcal{L}, \mathcal{A}=w(\mathcal{L})$, é um atrator compacto que atrai os conjuntos limitados de $\mathcal{U}$. Ele é maximal para a relação de inclusão.

\subsubsection{Lemas de Gronwall}

Dois Lemas de Gronwall que serão freqüentemente usados, são enunciados a seguir:

Lema clássico de Gronwall.- Sejam $g, h, y$ e $d y / d t$ funções localmente integráveis em $\left[t_{0}, \infty\right)$ satisfazendo

$$
\frac{d y}{d t}=g y+h, \quad \forall t \geq t_{0}
$$

Então para todo $t \geq t_{0}$ :

$$
y(t) \leq y\left(t_{0}\right) \exp \left[\int_{t_{0}}^{t} g(z) d z\right]+\int_{t_{0}}^{t} h(s) \exp \left[-\int_{t}^{s} g(z) d z\right] d s,
$$

A desigualdade (1.15) é a desigualdade clássica de Gronwall. Para nossos propósitos ela é útil para valores limitados de $t$ e não para $t \uparrow \infty$, já que propicia um crescimento exponencial para $y(t)$. Por exemplo com $y \geq 0, h \sim 0, g \sim 1$, ela dá $y(t) \leq y\left(t_{0}\right) e^{\left(t-t_{0}\right)}$.

Uma forma alternativa desta desigualdade, mediante hipóteses um pouco mais fortes, propicia uma estimativa uniforme para $y(t)$ em $t \geq t_{0}$ :

Lema uniforme de Gronwall.- Sejam $g, h$ e $y$ funções localmente integráveis em $\left(t_{0}, \infty\right)$, sendo também $y^{\prime}$ localmente integrável e satisfazendo (1.14) e

$$
\int_{t}^{t+r} g(s) d s \leq a_{1}, \quad \int_{t}^{t+r} h(s) d s \leq a_{2}, \quad \int_{t}^{t+r} y(s) d s \leq a_{3}, \quad t \geq t_{0}
$$

onde $r, a_{1}, a_{2}$ e $a_{3}$ são constantes positivas. Então,

$$
y(t+r) \leq\left(\frac{a_{3}}{r}+a_{2}\right) e^{a_{1}}, \quad \forall t \geq t_{0}
$$


Uma prova destes dois Lemas de Gronwall é dada em [66].

Também será muito usada é a desigualdade de Young:

Desigualdade de Young.- Seja $a, b, \epsilon>0, \quad 1<p<\infty, q=p /(p-1)$, então se verifica:

$$
a b \leq \frac{\epsilon}{p} a^{p}+\frac{1}{q \epsilon^{\frac{q}{p}}} b^{q} .
$$

\subsection{Limitação das soluções}

A existência e limitação das soluções das equações de água rasa (ainda no caso hiperbólico destas equações) são questões em aberto. Agoshkov et. al. [1] obtém a limitação de soluções para as equações de água rasa semi-parabólicas assumindo a existência, e a hipótese:

Hipótese 1.1 Se $\psi=h+z>0$, assuma-se que:

$$
\left|\nu \frac{\nabla \psi}{\psi} \cdot \nabla \vec{V}\right|<<|(\vec{V} \cdot \nabla) \vec{V}| .
$$

onde, $\vec{V}$ é a velocidade horizontal.

A hipótese $\psi=h+z>0$ é compativel com a física do problema e deve resultar de uma prova da existência de soluções (o que não é feito em [1]), enquanto que (1.19) é compatível com a seguinte análise de escala:

$$
\frac{\left[\nu \frac{\nabla \psi}{\psi} \cdot \nabla \vec{V}\right]}{[(\vec{V} \cdot \nabla) \vec{V}]} \sim \frac{\left.[\nu] \frac{[\psi]}{L[\psi]}\right] \frac{[\vec{V}]}{L}}{[\vec{V}] \frac{1}{L}[\vec{V}]} \sim \frac{[\nu]}{L[\vec{V}]} \sim f^{-1} L^{-2}[\nu] \sim \nu_{0}
$$

onde, $L$ é a escala horizontal, $f^{-1}$ é a escala temporal e $\nu_{0}$ é a viscosidade adimensionalizada (ver seção (1.3) para a descrição das escalas). A hipótese 1.1 é válida se $\nu_{0}$ é suficientemente pequena. 
Assumindo a Hipótese 1.1 pode-se obter a limitação das soluções do sistema (1.7) e (1.8) ( com $h=0)$. Com efeito, sob a hipótese 1.1 e considerando $\kappa$ da mesma ordem que $\nu$ (por exemplo $\kappa \leq \nu$ ) este sistema pode-se escrever como:

$$
\begin{aligned}
\frac{\partial \vec{V}}{\partial t}+\vec{V} \cdot \nabla \vec{V}+f \vec{k} \times \vec{V}+g \nabla \tilde{z}-\nu \Delta \vec{V}-(\nu+\kappa) \frac{\nabla \psi}{\psi} \cdot \nabla \vec{V} & =0 \\
\frac{\partial z}{\partial t}+\nabla \cdot(\psi \vec{V})-\kappa \Delta z & =F
\end{aligned}
$$

Fazendo o seguinte produto interno no espaço $L_{2}:((1.20), \psi \vec{V})+((1.21), g z)$, obtemos

$$
\begin{array}{r}
\left(\frac{\partial \vec{V}}{\partial t}, \psi \vec{V}\right)+(\vec{V} \cdot \nabla \vec{V}, \psi \vec{V})+f(\vec{k} \times \vec{V}, \psi \vec{V})+g(\nabla \tilde{z}, \psi \vec{V})-\nu(\Delta \vec{V}, \psi \vec{V})- \\
-(\nu+\kappa)\left(\frac{\nabla \psi}{\psi} \cdot \nabla \vec{V}, \psi \vec{V}\right)+g\left(\frac{\partial z}{\partial t}, z\right)+g(\nabla \cdot(\psi \vec{V}), z)-g \kappa(\Delta z, \psi z)=g(F, z)
\end{array}
$$

denotando $V^{2}=\vec{V} \cdot \vec{V}$, e usando as condições de contorno periódicas, os termos de (1.22) são avaliados como segue:

a1.- $f(\vec{k} \times \vec{V}, \psi \vec{V})=\int_{\Omega} f \psi(\vec{k} \times \vec{V}) \cdot \vec{V} d x=0$

a2.- $g(\nabla z, \psi \vec{V})+g(\nabla \cdot(\psi \vec{V}), z)=g(\nabla z, \psi \vec{V})-g(\psi \vec{V}, \nabla z)=0$

a3.- $g\left(\frac{\partial z}{\partial t}, z\right)=\frac{g}{2} \frac{\partial}{\partial t}|z|^{2}$

a4.-

$$
\begin{aligned}
(\vec{V} \cdot \nabla \vec{V}, \psi \vec{V}) & =\int_{\mathcal{Q}} \sum_{j, k=1}^{2} v_{j} \frac{\partial v_{k}}{\partial x_{j}} \psi v_{k} d x=\frac{1}{2} \int_{\mathcal{Q}} \sum_{j, k=1}^{2} \psi v_{j} \frac{\partial\left(v_{k}^{2}\right)}{\partial x_{j}} d x \\
& =-\frac{1}{2} \int_{\mathcal{Q}} \sum_{j, k=1}^{2} v_{k}^{2} \frac{\partial\left(\psi v_{j}\right)}{\partial x_{j}} d x=-\frac{1}{2} \int_{\mathcal{Q}} \sum_{j=1}^{2} \frac{\partial\left(\psi v_{j}\right)}{\partial x_{j}} \sum_{k=1}^{2} v_{k}^{2} d x \\
& =-\frac{1}{2} \int_{\mathcal{Q}}(\vec{V} \cdot \vec{V})(\nabla \cdot(\psi \vec{V})) d x
\end{aligned}
$$

a5.-

$$
\begin{aligned}
& -\nu(\Delta \vec{V}, \psi \vec{V})=-\nu \sum_{j=1}^{2} \int_{\mathcal{Q}}\left(\Delta v_{j}\right) \psi v_{j} d x=\nu \int_{\mathcal{Q}} \sum_{j=1}^{2} \nabla v_{j} \cdot \nabla\left(\psi v_{j}\right) d x \\
& \quad=\nu \int_{\mathcal{Q}} \sum_{j=1,2}\left(\psi \nabla v_{j} \cdot \nabla v_{j}+v_{j} \nabla v_{j} \cdot \nabla \psi\right) d x=\nu(\psi \nabla \vec{V}, \nabla \vec{V})+\nu(\nabla \psi \cdot \nabla \vec{V}, \vec{V})
\end{aligned}
$$


a6.-

$$
\begin{aligned}
\left(\frac{\partial \vec{V}}{\partial t}, \psi \vec{V}\right) & =\int_{\mathcal{Q}} \psi \vec{V} \cdot \frac{\partial \vec{V}}{\partial t} d x=\frac{1}{2} \int_{\mathcal{Q}} \psi \frac{\partial}{\partial t}(\vec{V} \cdot \vec{V}) d x \\
& =\frac{1}{2} \int_{\mathcal{Q}} \frac{\partial}{\partial t}(\psi \vec{V} \cdot \vec{V}) d x-\frac{1}{2} \int_{\mathcal{Q}}(\vec{V} \cdot \vec{V}) \frac{\partial z}{\partial t} d x \\
& =\frac{1}{2} \int_{\mathcal{Q}} \frac{\partial}{\partial t}(\psi \vec{V} \cdot \vec{V}) d x-\frac{1}{2} \int_{\mathcal{Q}} V^{2}(-\nabla \cdot(\psi \vec{V})+\kappa \Delta \tilde{z}+F) d x(\text { por }(1.21)) \\
& =\frac{1}{2} \frac{\partial}{\partial t}(\psi \vec{V}, \vec{V})+\frac{1}{2} \int_{\mathcal{Q}} V^{2}(\nabla \cdot(\psi \vec{V})) d x-\frac{\kappa}{2} \int_{\mathcal{Q}} V^{2} \Delta \tilde{z} d x-\frac{1}{2}\left(F, V^{2}\right) \\
& =\frac{1}{2} \frac{\partial}{\partial t}(\psi \vec{V}, \vec{V})+\frac{1}{2} \int_{\mathcal{Q}} V^{2}(\nabla \cdot(\psi \vec{V})) d x+\kappa(\nabla \psi \cdot \nabla \vec{V}, \vec{V})-\frac{1}{2}\left(F, V^{2}\right)
\end{aligned}
$$

na ultima igualdade temos usado:

$$
\begin{aligned}
\kappa(\nabla \psi \cdot \nabla \vec{V}, \vec{V}) & =\kappa \int_{\mathcal{Q}} \sum_{j=1}^{2} v_{j}\left(\nabla \psi \cdot \nabla v_{j}\right) d x=\frac{\kappa}{2} \int_{\mathcal{Q}} \sum_{j=1}^{2} \nabla \psi \cdot \nabla\left(v_{j}^{2}\right) d x \\
& =-\frac{\kappa}{2} \int_{\mathcal{Q}} \sum_{j=1}^{2} \Delta \psi\left(v_{j}^{2}\right) d x=-\frac{\kappa}{2} \int_{\mathcal{Q}} V^{2} \Delta z d x
\end{aligned}
$$

Substituindo (a1)-(a6) na equação (1.22) resulta:

$$
\begin{aligned}
\frac{1}{2} \int_{\mathcal{Q}} \frac{\partial}{\partial t}\left(\psi V^{2}\right) d x+\frac{g}{2} \frac{\partial}{\partial t}|z|^{2}+\nu(\psi \nabla \vec{V} & , \nabla \vec{V})+g \kappa|\nabla z|^{2}=g(F, z)+\left(F, V^{2}\right) \\
& \leq g|F||z|+|F|_{\infty}|\vec{V}|^{2} \\
& \leq \frac{g \kappa}{2}|z|^{2}+\frac{g}{2 \kappa}|F|^{2}+|F|_{\infty}|\vec{V}|^{2} \\
& \leq \frac{g \kappa}{2}|z|^{2}+\frac{g}{2 \kappa}|F|^{2}+c_{1}|F|_{\infty}|\nabla \vec{V}|^{2}
\end{aligned}
$$

(Pela desigualdade de Poincaré )

Para estimar o termo $c_{1}|F|_{\infty}|\nabla \vec{V}|^{2}$ a seguinte hipótese é formulada:

Hipótese 1.2 Assuma-se que:

$$
|F|_{\infty} \leq \frac{\nu \psi_{0}}{2 c_{1}}
$$

onde, $\psi_{0}$ é uma constante, e $\psi \geq \psi_{0}>0$.

Considerando a Hipótese (1.2) na desigualdade anterior obtemos:

$$
\frac{1}{2} \frac{\partial}{\partial t} \int_{\mathcal{Q}}\left(\psi V^{2}\right) d x+\frac{g}{2} \frac{\partial}{\partial t}|z|^{2}+\frac{\nu}{2} \psi_{0}|\nabla \vec{V}|^{2}+\frac{1}{2} g \kappa|\nabla z|^{2} \leq \frac{g}{2 \kappa}|F|^{2}
$$


Integrando de $\tau=0$ até $\tau=t$, para $0 \leq t \leq T$, temos a seguinte estimativa:

\section{Estimativa 1.1}

$$
\begin{aligned}
\int_{\mathcal{Q}} \psi V^{2} d \Omega+g|z|^{2} & +\nu \int_{\tau=0}^{t} \psi_{0}|\nabla \vec{V}|^{2} d \tau+g \kappa \int_{\tau=0}^{t}|\nabla \tilde{z}|^{2} d \tau \\
& \leq \int_{\Omega} \psi(0) V^{2}(0) d \Omega+g|z(0)|^{2}+\frac{g}{\kappa} T|F|^{2}
\end{aligned}
$$

A Estimativa 1.1 fornece uma limitação para as soluções de (1.20)-(1.21), e usando a Hipótese (1.1) também fornece uma limitação para as soluções de (1.7)-(1.8). Observe-se que a Hipótese 1.2 não é necessária se a forçante $F$ não for considerada em (1.21), enquanto a hipótese $\psi=H+z>0$ é fundamental na obtenção desta estimativa, mas como foi observado acima, a prova da positividade de $\psi$ deveria possivelmente resultar de uma prova da existência das soluções.

Esta estimativa foi apresentada seguindo as hipóteses para a limitação das equações de água rasa semi-parabólicas obtidas por Agoshkov [1]. No caso geral assumimos a limitação das equações de água rasa (1.7) e (1.8), a qual é necessária para obter estimativas de erro das variedades inerciais para estas equações.

\subsection{As equações adimensionalizadas e estimativas a priori}

As equações (1.7) e (1.8) são adimensionalizadas como em Lorenz [46]. Através das transformações:

$$
t \rightarrow f^{-1} t ; \quad(x, y) \rightarrow(L x, L y), \quad z \rightarrow f^{2} L^{2} g^{-1} z .
$$

resulta a seguinte forma adimensional em $D=[0,2 \pi] \times[0,2 \pi]$ :

$$
\begin{aligned}
& \frac{\partial \vec{V}}{\partial t}=-(\vec{V} \cdot \nabla) \vec{V}-\vec{k} \wedge \vec{V}-\nabla z+\nu_{0} \Delta \vec{V} \\
& \frac{\partial z}{\partial t}=-\nabla \cdot\left(\left(g_{0}+z\right) \vec{V}\right)+\kappa_{0} \Delta z+F
\end{aligned}
$$


onde em (1.24), (1.25) temos: $\nu_{0}=f^{-1} L^{-2} \nu, \quad \kappa_{0}=f^{-1} L^{-2} \kappa, \quad h_{0}=g_{0}=f^{-2} L^{-2} g h$, $\vec{V} \rightarrow f L \vec{V}, \quad F^{\rightarrow} f^{3} L^{2} g^{-1} F, \quad z \rightarrow f^{2} L^{2} g^{-1} z$.

Os espaçõs $H$ e $V$ são agora definidos por $H=H_{p}^{0}(D)$ e $V=H_{p}^{1}(D)$.

Aqui será conveniente utilizar uma notação mais compacta. Definimos: $\xi=\left(\begin{array}{c}\vec{V} \\ z\end{array}\right)$, $|\xi|=\left\{|\vec{V}|^{2}+|z|^{2}\right\}^{1 / 2},\|\xi\|=\left\{\|\vec{V}\|^{2}+\|z\|^{2}\right\}^{1 / 2}$.

Na literatura ([47], [46], [13], [7]) é usual tomar $\kappa_{0}=\nu_{0}$. Aqui admitiremos o caso mais geral: com a hipótese (1.1), teremos

$$
0 \leq \kappa_{0} \leq \nu_{0}
$$

Com base na discussão da seção 1.2 sobre a limitação das soluções do sistema (1.7)(1.8), assumimos no que segue a seguinte limitação no tempo para a solução das equações adimencionalizadas (1.24), (1.25):

$$
\sup _{0 \leq t \leq T}|\xi(t)| \leq \delta=\delta(T)<\infty
$$

No que segue desta seção, consideramos os dados iniciais $\xi(0)$ em $\mathbb{H} \times H$, e o termo forçante $F$ em $H$. Nas próximas seções as desigualdades de Poincaré $(\mathbf{D} 1-\mathbf{D} 3)$ e de Sobolev (E1 - E4) serão aplicadas no domínio D.

\subsubsection{Existência de conjuntos absorventes em $\mathbb{H} \times H$ para a dinâmica do sis- tema (1.24)-(1.25).}

Será útil ainda observar que as equações (1.24) e (1.25), através de uma mudança de escala por uma constante positiva $\lambda(\vec{V} \rightarrow \lambda \vec{V}, z \rightarrow \lambda z)$, tomam a forma :

$$
\begin{aligned}
& \frac{\partial \vec{V}}{\partial t}=-\lambda \vec{V} \cdot \nabla \vec{V}-k \wedge \vec{V}-\nabla z+\nu_{0} \Delta \overrightarrow{\vec{V}} \\
& \frac{\partial z}{\partial t}=-\nabla \cdot\left(\left(g_{0}+\lambda z\right) \vec{V}\right)+\kappa_{0} \Delta z+\frac{F}{\lambda}
\end{aligned}
$$

Fazendo o produto escalar de (1.24') e (1.25') por $g_{0} \vec{V}$ e $z$ respectivamente e somando, 
vemos que os termos $-\left(\nabla z, g_{0} \vec{V}\right)$ e $-\left(\nabla \cdot g_{0} \vec{V}, z\right)$ se cancelam devido às condições de contorno periódicas, levando à seguinte equação:

$$
\begin{aligned}
\frac{1}{2} \frac{d}{d t}\left(g_{0}|\vec{V}|^{2}+|z|^{2}\right)+\nu_{0} g_{0}|\nabla \vec{V}|^{2}+\kappa_{0}|\nabla z|^{2}= & -\lambda\left(\vec{V} \cdot \nabla \vec{V}, g_{0} \vec{V}\right)-\lambda(\nabla \cdot(z \vec{V}), z)+ \\
& +\lambda^{-1}(F, z)
\end{aligned}
$$

Usando (1.26) temos:

$$
\begin{aligned}
\frac{1}{2} \frac{d}{d t}\left(g_{0}|\vec{V}|^{2}+|z|^{2}\right)+\kappa_{0}\left(g_{0}\|\vec{V}\|^{2}+\|z\|^{2}\right) \leq & \lambda g_{0}|(\vec{V} \cdot \nabla \vec{V}, \vec{V})|+\lambda|(\nabla \cdot(z \vec{V}), z)|+ \\
& +\lambda^{-1}|(F, z)|
\end{aligned}
$$

Usando a estimativa E1, a desigualdade de Young e se tomarmos

$$
\lambda=\min \left\{\frac{\kappa_{0}}{4 c_{1} \Delta+1} ; \frac{g_{0}^{1 / 4} \kappa_{0}}{\left(27 c_{1}^{4} \Delta^{4}+1\right)}\right\}
$$

os termos da direita de (1.27) são estimados como segue:

a.- $\lambda g_{0}|((\vec{V} \cdot \nabla) \vec{V}, \vec{V})| \leq \lambda g_{0} c_{1}|\vec{V}|\|\vec{V}\|^{2} \leq \lambda g_{0} c_{1}|\xi|\|\vec{V}\|^{2} \leq \frac{1}{4} g_{0} \kappa_{0}\|\vec{V}\|^{2}$

b.- $\lambda|(\nabla \cdot(z \vec{V}), z)|=\lambda|(z \nabla z, \vec{V})| \leq c_{1} \lambda|\vec{V}|^{1 / 2}\|\vec{V}\|^{1 / 2}|z|^{1 / 2}\|z\|^{3 / 2} \leq c_{1} \lambda|\xi|\|\vec{V}\|^{1 / 2}\|z\|^{3 / 2}$

$$
\leq \frac{1}{4} \kappa_{0}\|z\|^{2}+\frac{27}{4} \kappa_{0}^{-3} c_{1}^{4} \lambda^{4}|\xi|^{4}\|v\|^{4} \leq \frac{1}{4} \kappa_{0}\|z\|^{2}+\frac{1}{4} g_{0} \kappa_{0}\|\vec{V}\|^{2}
$$

(onde foi usada a desigualdade de Young $\operatorname{com} p=4 / 3, q=4, \epsilon=\kappa_{0} / 3$ )

c. $-\lambda^{-1}|F||z| \leq \lambda^{-1} \lambda_{1}^{-1 / 2}|F|\|z\| \leq \frac{1}{4} \kappa_{0}\|z\|^{2}+\kappa_{0}^{-1} \lambda_{1}^{-1} \lambda^{-2}|F|^{2}$

Substituindo (a)-(c) em (1.27) e simplificando, obtemos

$$
\frac{d}{d t}\left(g_{0}|\vec{V}|^{2}+|z|^{2}\right)+\kappa_{0}\left(g_{0}\|\vec{V}\|^{2}+\|z\|^{2}\right) \leq \frac{2|F|^{2}}{\kappa_{0} \lambda_{1} \lambda^{2}}
$$

Por reescalonamento $(\lambda \vec{V} \rightarrow \vec{V}, \lambda z \rightarrow z)$,

$$
\frac{d}{d t}\left(g_{0}|\vec{V}|^{2}+|z|^{2}\right)+\kappa_{0}\left(g_{0}\|\vec{V}\|^{2}+\|z\|^{2}\right) \leq \frac{2|F|^{2}}{\kappa_{0} \lambda_{1}}
$$

e portanto

$$
\frac{d}{d t}\left(g_{0}|\vec{V}|^{2}+|z|^{2}\right)+\lambda_{1} \kappa_{0}\left(g_{0}|\vec{V}|^{2}+|z|^{2}\right) \leq \frac{2|F|^{2}}{\kappa_{0} \lambda_{1}}
$$


Segue então do lema clássico de Gronwall, para $t \in[0, T]$ :

$$
g_{0}|\vec{V}(t)|^{2}+|z(t)|^{2} \leq\left(g_{0}|\vec{V}(0)|^{2}+|z(0)|^{2}\right) e^{-\kappa_{0} \lambda_{1} t}+\frac{2|F|^{2}}{\kappa_{0}^{2} \lambda_{1}^{2}}\left(1-e^{-\kappa_{0} \lambda_{1} t}\right),
$$

e definindo, $\theta_{1}=\left\{\begin{array}{ll}g_{0}^{-1}, & \text { se } 0<g_{0}<1 \\ g_{0}, & \text { se } g_{0} \geq 1\end{array}, \quad\right.$ e $\quad \theta_{2}=\left\{\begin{array}{ll}g_{0}^{-1}, & \text { se } 0<g_{0}<1 \\ 1, & \text { se } g_{0} \geq 1\end{array}\right.$, obtemos,

$$
|\xi(t)|^{2} \leq \theta_{1}|\xi(0)|^{2} e^{-\kappa_{0} \lambda_{1} t}+\frac{2 \theta_{2}|F|^{2}}{\kappa_{0}^{2} \lambda_{1}^{2}}\left(1-e^{-\kappa_{0} \lambda_{1} t}\right)
$$

e, portanto

\section{Estimativa 1.2}

$$
\varlimsup_{t \rightarrow \infty}|\xi(t)| \leq \rho_{0}, \quad \rho_{0}=\frac{\sqrt{2 \theta_{2}}|F|}{\kappa_{0} \lambda_{1}}
$$

Inferimos da Estimativa (1.2): as bolas $B(0, \rho)$ de $\mathbb{H} \times H$, com $\rho \geq \rho_{0}$, são positivamente invariantes para a dinâmica $S(t)$ das equações (1.24)-(1.25), e essas bolas são absorventes em $\mathbb{H} \times H$ para qualquer $\rho>\rho_{0}$. Pois tomando $\rho_{0}^{\prime} \geq \rho_{0}$, qualquer limitado $\mathcal{L}$ de $H$ está contido em alguma bola $B(0, R)$, e da desigualdade (1.29) sai que $S(t) \mathcal{L} \subset B\left(0, \rho_{0}^{\prime}\right)$ para $t \geq t_{0}\left(\mathcal{L}, \rho_{0}^{\prime}\right)$, onde $t_{0}=\frac{1}{\kappa_{0} \lambda_{1}} \log \frac{R^{2}}{\rho_{0}^{\prime 2}-\rho_{0}^{2}}$, confirmando que órbitas começando em $\mathcal{L}$ entram necessariamente em $B\left(0, \rho_{0}^{\prime}\right)$ após $t_{0}$.

Agora de (1.28) deduzimos, após integração em $t$ que

$$
\kappa_{0} \int_{t}^{t+r}\|\xi\|^{2} d s \leq \theta_{1}|\xi(t)|^{2}+\frac{2 \theta_{2} r}{\kappa_{0} \lambda_{1}}|F|^{2}, \quad \forall r>0 .
$$

Temos ainda:

\section{Estimativa 1.3}

$$
\varlimsup_{t \rightarrow \infty} \int_{t}^{t+r}\|\xi\|^{2} d s \leq \frac{\theta_{1}}{\kappa_{0}} \varlimsup_{t \rightarrow \infty}|\xi(t)|^{2}+\frac{2 r \theta_{2}}{\kappa_{0}^{2} \lambda_{1}}|F|^{2} \leq \frac{2 \theta_{1} \theta_{2}}{\kappa_{0}^{3} \lambda_{1}^{2}}|F|^{2}+\frac{2 r \theta_{2}}{\kappa_{0}^{2} \lambda_{1}}|F|^{2}
$$


Mais ainda, se $\xi_{0} \in \mathcal{L} \subset B(0, R)$ e $t \geq t_{0}\left(\mathcal{L}, \rho_{0}^{\prime}\right)$, então:

$$
\int_{t}^{t+r}\|\xi\|^{2} d s \leq \frac{2 \theta_{2} r}{\kappa_{0}^{2} \lambda_{1}}|F|^{2}+\frac{\theta_{1}}{\kappa_{0}} \rho_{0}^{\prime 2}
$$

e em particular para $t=0, r=T$, de (1.30) obtemos

$$
\kappa_{0} \int_{0}^{T}\|\xi\|^{2} \leq \theta_{1}|\xi(0)|^{2}+\frac{2 \theta_{2} T}{\kappa_{0} \lambda_{1}}|F|^{2} .
$$

1.3.2 Existência de conjuntos absorventes em $\mathbb{V} \times V$ e atratores em $\mathbb{H} \times H$ para a dinâmica do sistema (1.24)-(1.25)

Observando que $A_{0} \operatorname{div} \vec{V}=\operatorname{div}\left(A_{0} \vec{V}\right)$ e tomando o produto escalar de (1.24) e (1.25) por $g_{0} A_{0} \vec{V}$ e $A_{0} z$ respectivamente, vemos que:

$$
\begin{aligned}
\frac{1}{2} \frac{d}{d t}\left(g_{0}\|\vec{V}\|^{2}+\|z\|^{2}\right)+g_{0} \nu_{0}\left|A_{0} \vec{V}\right|^{2}+\kappa_{0}\left|A_{0} z\right|^{2}= & -g_{0}\left(\vec{V} \cdot \nabla \vec{V}, A_{0} \vec{V}\right)-\left(\nabla \cdot(z \vec{V}), A_{0} z\right) \\
& +\left(F, A_{0} z\right) .
\end{aligned}
$$

Considerando (1.26), temos:

$$
\begin{array}{r}
\frac{1}{2} \frac{d}{d t}\left(g_{0}\|\vec{V}\|^{2}+\|z\|^{2}\right)+\kappa_{0}\left(g_{0}\left|A_{0} \vec{V}\right|^{2}+\left|A_{0} z\right|^{2}\right) \leq g_{0}\left|\left(\vec{V} \cdot \nabla \vec{V}, A_{0} \vec{V}\right)\right|+ \\
+\left|\left(\nabla \cdot(z \vec{V}), A_{0} z\right)\right|+\left|\left(F, A_{0} z\right)\right| .
\end{array}
$$

Estimamos os termos do lado direito de (1.32) como segue:

a.- usando (E2) e a desigualdade de Young com $p=4 / 3, q=4, \epsilon=2 \kappa_{0} / 3$ temos,

$$
\begin{aligned}
g_{0}\left|\left((\vec{V} \cdot \nabla) \vec{V}, A_{0} \vec{V}\right)\right| & \leq g_{0} c_{2}|\vec{V}|^{1 / 2}\|\vec{V}\|\left|A_{0} \vec{V}\right|^{3 / 2} \\
\leq & \frac{1}{2} g_{0} \kappa_{0}\left|A_{0} \vec{V}\right|^{2}+\frac{27}{32} g_{0} c_{2}^{4} \kappa_{0}^{-3}|\vec{V}|^{2}\|\vec{V}\|^{4} \\
\leq & \frac{1}{2} g_{0} \kappa_{0}\left|A_{0} \vec{V}\right|^{2}+\frac{27}{32} c_{2}^{4} \kappa_{0}^{-3}|\xi|^{2}\|\xi\|^{2}\left(g_{0}\|\vec{V}\|^{2}+\|z\|^{2}\right)
\end{aligned}
$$

b.- usando (E2) e a desigualdade de Young com $p=4 / 3, q=4, \epsilon=4 \kappa_{0} / 9$ temos,

$$
\begin{aligned}
\left|\left(z \nabla \cdot \vec{V}, A_{0} z\right)\right| & \leq c_{2}|z|^{1 / 2}\|\vec{V}\|\left|A_{0} z\right|^{3 / 2} \leq \frac{1}{3} \kappa_{0}\left|A_{0} z\right|^{2}+\frac{729}{256} c_{2}^{4} \kappa_{0}^{-3}|z|^{2}\|\vec{V}\|^{4} \\
& \leq \frac{1}{3} \kappa_{0}\left|A_{0} z\right|^{2}+\frac{729}{256} c_{2}^{4} \kappa_{0}^{-3} g_{0}^{-1}|\xi|^{2}\|\xi\|^{2}\left(g_{0}\|\vec{V}\|^{2}+\|z\|^{2}\right)
\end{aligned}
$$


c.- usando (E2) e desigualdade de Young com $p=q=2, \epsilon=2 \kappa_{0} / 3$ e $\epsilon=g_{0} \kappa_{0}$ temos,

$$
\begin{aligned}
\left|\left(\vec{V} \cdot \nabla z, A_{0} z\right)\right| & \leq c_{2}|\vec{V}|^{1 / 2}\|z\|\left|A_{0} \vec{V}\right|^{1 / 2}\left|A_{0} z\right| \\
\leq & \frac{1}{3} \kappa_{0}\left|A_{0} z\right|^{2}+\frac{3}{4} c_{2}^{2} \kappa_{0}^{-1}|\vec{V}|\|z\|^{2}\left|A_{0} \vec{V}\right| \\
\leq & \frac{1}{3} \kappa_{0}\left|A_{0} z\right|^{2}+\frac{1}{2} g_{0} \kappa_{0}\left|A_{0} \vec{V}\right|^{2}+\frac{9}{32} c_{2}^{4} \kappa_{0}^{-3} g_{0}^{-1}|\vec{V}|^{2}\|z\|^{4} \\
\leq & \frac{1}{3} \kappa_{0}\left|A_{0} z\right|^{2}+\frac{1}{2} g_{0} \kappa_{0}\left|A_{0} \vec{V}\right|^{2}+\frac{9}{32} c_{2}^{4} \kappa_{0}^{-3} g_{0}^{-1}|\xi|^{2}\|\xi\|^{2}\left(g_{0}\|\vec{V}\|^{2}+\|z\|^{2}\right)
\end{aligned}
$$

d.- $\mid\left(F,\left.A_{0} z|\leq| F|| A_{0} z\left|\leq \frac{1}{3} \kappa_{0}\right| A_{0} z\right|^{2}+\frac{3}{4} \kappa_{0}^{-1}|F|^{2}\right.$

Substituindo (a)-(d) em (1.32), obtemos após simplificações,

$$
\frac{d}{d t}\left(g_{0}\|\vec{V}\|^{2}+\|z\|^{2}\right) \leq G_{1}\left(g_{0}\|\vec{V}\|^{2}+\|z\|^{2}\right)+G_{2}
$$

onde

$$
\begin{aligned}
G_{1} & =\theta_{3}|\xi|^{2}\|\xi\|^{2}, \quad \text { com } \theta_{3}=\frac{\left(216+801 g_{0}^{-1}\right)}{128} c_{2}^{4} \kappa_{0}^{-3} \\
\text { e } G_{2} & =\frac{3}{2 \kappa_{0}}|F|^{2} .
\end{aligned}
$$

Para T suficientemente grande e $t \geq T(\epsilon)$, avaliamos $G_{1}$ e $G_{2}$. Considerando a Estimativa (1.2) e a desigualdade (1.30) temos

$$
\begin{aligned}
\int_{t}^{t+r} G_{1} d \tau & \leq \theta_{3} \int_{t}^{t+r}|\xi|\|\xi\|^{2} d \tau \leq \frac{2 \theta_{2} \theta_{3}}{\kappa_{0}^{2} \lambda_{1}^{2}}(1+\epsilon)|F|^{2} \int_{t}^{t+r}\|\xi\|^{2} d \tau \\
& \leq \frac{2 \theta_{2} \theta_{3}}{\kappa_{0}^{2} \lambda_{1}^{2}}(1+\epsilon)\left(\frac{2 \theta_{1} \theta_{2}}{\kappa_{0}^{3} \lambda_{1}^{2}}(1+\epsilon)+\frac{2 \theta_{2} r}{\kappa_{0}^{2} \lambda_{1}}\right)|F|^{4}=a_{1}(r, \epsilon), \\
\int_{t}^{t+r} G_{2} d \tau & \leq \frac{3 r}{2 \kappa_{0}}|F|^{2}=a_{2}(r, \epsilon) \mathrm{e} \\
\int_{t}^{t+r}\|\xi(\tau)\|^{2} d \tau & \leq\left(\frac{2 \theta_{1} \theta_{2}}{\kappa_{0}^{3} \lambda_{1}^{2}}(1+\epsilon)+\frac{2 \theta_{2} r}{\kappa_{0}^{2} \lambda_{1}}\right)|F|^{2}=a_{3}(r, \epsilon) .
\end{aligned}
$$

Aplicando o lema uniforme de Gronwall obtemos

$$
g_{0}\|\vec{V}\|^{2}+\|z\|^{2} \leq\left(\frac{a_{3}}{r}+a_{2}\right) e^{a_{1}} \quad \text { para } t \geq T(\epsilon)+r
$$

o que implica

$$
\|\xi(t)\|^{2} \leq \theta_{2}\left(\frac{a_{3}}{r}+a_{2}\right) e^{a_{1}}=\rho_{1}^{2}(r, \epsilon), \quad \text { para } t \geq T(\epsilon)+r .
$$


Escolhendo $\epsilon=1 / 2$ e $r=1 / 4$ temos :

Estimativa 1.4 Para $t \geq T\left(\frac{1}{2}\right)+\frac{1}{4}$ se verifica

$$
\|\xi(t)\| \leq \hat{M}_{1}\left(\kappa_{0}, F\right)
$$

onde $\hat{M}_{1}=\rho_{1}(1 / 4,1 / 2)$.

Na prova pode-se considerar $u_{0} \in \mathcal{L}, \mathcal{L}$ algum limitado de $\mathbb{H} \times H$, e $t \geq t_{0}\left(\mathcal{L}, \rho_{0}^{\prime}\right)$, obtendo novamente (1.34) para $t>t_{0}\left(\mathcal{L}, \rho_{0}^{\prime}\right)+r$. Agora fixando $r>0,(1.34)$ permite-nos concluir que a bola $B\left(0, \rho_{1}\right)$ é um conjunto absorvente em $\mathbb{V} \times V$ para a dinâmica $S(t)$. E mais, para qualquer limitado $\mathcal{L}$ de $\mathbb{H} \times H, \bigcup_{t \geq t_{0}\left(\mathcal{L}, \rho_{0}^{\prime}\right)+r} S(t) \mathcal{L}$ esta contido em $B\left(0, \rho_{1}\right)$ de $\mathbb{V} \times V$, sendo portanto relativamente compacto em $\mathbb{H} \times H$. Ou seja a propriedade (1.13) está satisfeita para a dinâmica $S(t)$. Então, pelo Teorema (1.1), o sistema dinâmico associado ao sistema (1.24)-(1.25) (isto é $S(t)$ ) possui um atrator universal $\mathcal{A}$ em $H$. Este atrator contém todos os conjuntos $w$-limite correspondente a todos os estados iniciais $u_{0} \in \mathbb{H} \times H$, e portanto constitui-se numa descrição universal do escoamento 'a longo prazo'. 


\subsection{Estimativas de maior ordem}

Nossa análise para a construção da Variedade Inercial Aproximada (VIA) para (1.24), (1.25) será baseada no método de Heywood e Rannacher dado em [31], [32]. Isto nos levará às estimativas necessárias, exceto para tempos pequenos. Para obter estimativas no último caso impomos também as hipóteses $|-\Delta F|<\infty$, e $\left|(-\Delta)^{3 / 2} \xi(0)\right|<\infty$, permitindo obter também estimativas de erro para a parte transiente das equações de água rasa. Em trabalhos prévios, referentes às equações de Navier-Stokes, obtém-se estimativas de erro para o comportamento a longo prazo (ver por exemplo [23], [24], [25], [20], [39], [40], [37], [26], [61], [62], [63], [69], [60], [70]), enquanto que em [32] tem-se estimativas de erro para as equações de Navier-Stokes para $t>0$.

Como no modelo de água-rasa o termo de pressão é eliminado e por estarmos impondo condições de contorno periódicas, não se apresenta nenhuma dificuldade referente a condições de compatibilidade envolvendo o termo de pressão e dados iniciais, como no caso das equações de Navier-Stokes, sob condições de contorno de não-deslizamento.

A teoria de VIA foi bem estudada por Liu em [45], assumindo hipóteses adicionais de regularidade sobre $F$, para o caso das equações de Navier-Stokes.

A presente seção é dedicada ao estabelecimento de estimativas técnicas essenciais para a teoria das variedades inerciais aproximadas. De fato assumindo que o modelo de Lorenz ( (1.24), (1.25)) tem soluções globais satisfazendo a condição

$$
\|\xi(t)\| \leq M_{1}, \quad t \in[0, \infty)
$$

para alguma constante $M_{1}$, as seguintes estimativas serão estabelecidas:

$$
|-\Delta \xi(t)| \leq M_{2}, \quad\left|\xi_{t}(t)\right| \leq M_{3}, \quad\left\|\xi_{t}(t)\right\| \leq M_{5}, \quad \forall t>0
$$

\subsubsection{Estimativas para $\xi$ em $\mathbb{H}_{p}^{2} \times H_{p}^{2}$, e para $\xi_{t}$ em $\mathbb{H} \times H$}

Estas estimativas são resumidas no seguinte Lema:

Lema $1.1 \mathrm{Se}|-\Delta \xi(0)|<\infty$, e $\|\xi(t)\|$ é limitada no tempo, então $|-\Delta \xi(t)|$ e $\left|\xi_{t}(t)\right|$ também o são. 


\section{Prova.-}

Vamos separar a demonstração por etapas, iniciando com:

Lema 1.1.i: Para todo $\alpha, t, \in(0, \infty)$, existem constantes $K_{1}, K_{2}$ tais que:

$$
\kappa_{0} e^{-\alpha t} \int_{0}^{t} e^{\alpha \tau}|-\Delta \xi(\tau)|^{2} d \tau \leq K_{1} \alpha^{-1}+M_{1}^{2}=K_{2}
$$

Prova.-

Tomando o produto escalar de (1.24), (1.25) com respeito a $-\Delta \xi$ obtemos:

$$
\begin{aligned}
\frac{1}{2} \frac{d}{d t}\|\xi\|^{2}+\kappa_{0}|-\Delta \xi|^{2} \leq & |(\vec{V} \cdot \nabla \vec{V},-\Delta \vec{V})+|(\nabla z,-\Delta \vec{V}) \mid+ \\
& \left.+\mid \nabla \cdot\left(\left(g_{0}+z\right) \vec{V}\right),-\Delta z\right)|+|(F,-\Delta z) \mid .
\end{aligned}
$$

Com o uso das estimativas (E2), (D1) e (1.35), além da desigualdade de Young obtemos:

$$
\begin{gathered}
\text { a.- }|(\vec{V} \cdot \nabla \vec{V},-\Delta \vec{V})| \leq c_{2}|\vec{V}|^{1 / 2}\|\vec{V}\||-\Delta \vec{V}|^{3 / 2} \leq c_{2} \lambda_{1}^{-1 / 4} M_{1}^{3 / 2}|-\Delta \vec{V}|^{3 / 2} \\
\leq \frac{\kappa_{0}}{8}|-\Delta \vec{V}|^{2}+54 \kappa_{0}^{-3} c_{2}^{4} \lambda_{1}^{-1} M_{1}^{6}
\end{gathered}
$$

(aplicando a desigualdade de Young com: $p=\frac{4}{3}, q=4, \epsilon=\frac{\kappa_{0}}{6}$ ).

b.- $|(\nabla z,-\Delta \vec{V})| \leq|| z|||-\Delta \vec{V}| \leq M_{1}|-\Delta \vec{V}| \leq \frac{\kappa_{0}}{8}|-\Delta \vec{V}|^{2}+2 \kappa_{0}^{-1} M_{1}^{2}$

(aplicando a desigualdade de Young com: $p=2, q=2, \epsilon=\frac{\kappa_{0}}{4}$ ).

c. $|(\vec{V} \cdot \nabla z,-\Delta z)| \leq c_{2}|\vec{V}|^{1 / 2}|-\Delta \vec{V}|^{1 / 2}|| z \||-\Delta z| \leq c_{2} \lambda_{1}^{-1 / 4} M_{1}^{3 / 2}|-\Delta \xi|^{3 / 2}$

$$
\leq \frac{\kappa_{0}}{4}|-\Delta \xi|^{2}+\frac{27}{4} \kappa_{0}^{-3} c_{2}^{4} \lambda_{1}^{-1} M_{1}^{6}
$$

(aplicando a desigualdade de Young com: $p=\frac{4}{3}, q=4, \epsilon=\frac{\kappa_{0}}{3}$ ).

d.- $|(z \nabla \cdot \vec{V},-\Delta z)| \leq c_{2}|z|^{1 / 2}|-\Delta z|^{1 / 2}|| \vec{V}||-\Delta z\left|\leq c_{2} \lambda_{1}^{-1 / 4} M_{1}^{3 / 2}\right|-\left.\Delta z\right|^{3 / 2}$

$$
\leq \frac{\kappa_{0}}{12}|-\Delta z|^{2}+\frac{9^{3}}{4} \kappa_{0}^{-3} \lambda_{1}^{-1} c_{2}^{4} M_{1}^{6}
$$

(aplicando a desigualdade de Young com: $p=\frac{4}{3}, q=4, \epsilon=\frac{\kappa_{0}}{9}$ ).

$$
\text { e.- } \begin{aligned}
g_{0}|(\nabla \cdot \vec{V},-\Delta z)| & \leq g_{0}|\nabla \cdot \vec{V}||-\Delta z| \leq \sqrt{2} g_{0}|| \vec{V}|||-\Delta z| \leq \sqrt{2} g_{0} M_{1}|-\Delta z| \\
\leq & \frac{\kappa_{0}}{12}|-\Delta z|^{2}+6 \kappa_{0}^{-1} g_{0}^{2} M_{1}^{2}
\end{aligned}
$$


(aplicando a desigualdade de Young com: $p=2, q=2, \epsilon=\frac{\kappa_{0}}{6}$ ).

f.- $|(F,-\Delta z)| \leq|F||-\Delta z| \leq \frac{\kappa_{0}}{12}|-\Delta z|^{2}+3 \kappa_{0}^{-1}|F|^{2}$

(aplicando a desigualdade de Young com: $p=2, q=2, \epsilon=\frac{\kappa_{0}}{6}$ ).

Substituindo (a)-(f) em (1.37) resulta:

$$
\begin{aligned}
\frac{1}{2} \frac{d}{d t}|| \xi \|^{2}+\kappa_{0}|-\Delta \xi|^{2} & \leq \frac{\kappa_{0}}{4}\left(|-\Delta \vec{V}|^{2}+|-\Delta z|^{2}\right)+\frac{\kappa_{0}}{4}|-\Delta \xi|^{2}+\frac{1}{2} K_{1} \\
& \leq \frac{\kappa_{0}}{2}|-\Delta \xi|^{2}+\frac{1}{2} K_{1}
\end{aligned}
$$

Logo,

$$
\frac{d}{d t}\|\xi\|^{2}+\kappa_{0}|-\Delta \xi|^{2} \leq K_{1}
$$

onde

$$
K_{1}=486 \kappa_{0}^{-3} c_{2}^{4} \lambda_{1}^{-1} M_{1}^{6}+4 \kappa_{0}^{-1} M_{1}^{2}\left(1+3 g_{0}^{2}\right)+6 \kappa_{0}^{-1}|F|^{2}
$$

Agora multiplicando (1.38) por $e^{\alpha t}$, para algum $\alpha>0$,

$$
\frac{d}{d t}\left(e^{\alpha t}\|\xi\|^{2}\right)+\kappa_{0} e^{\alpha t}|-\Delta \xi|^{2} \leq K_{1} e^{\alpha t}+\alpha e^{\alpha t}\|\xi\|^{2} \leq K_{1} e^{\alpha t}+\alpha M_{1}^{2} e^{\alpha t}
$$

Integrando com respeito a $t$ obtemos:

$$
\begin{aligned}
e^{\alpha t}\|\xi\|^{2}+\kappa_{0} \int_{0}^{t} e^{\alpha \tau}|-\Delta \xi(\tau)|^{2} d \tau & \leq\|\xi(0)\|^{2}+K_{1} \alpha^{-1}\left(e^{\alpha t}-1\right)+M_{1}^{2}\left(e^{\alpha t}-1\right) \\
& \leq K_{1} \alpha^{-1} e^{\alpha t}+M_{1}^{2} e^{\alpha t}
\end{aligned}
$$

e multiplicando por $e^{-\alpha t}$ :

$$
\kappa_{0} e^{-\alpha t} \int_{0}^{t} e^{\alpha \tau}|-\Delta \xi(\tau)|^{2} d \tau \leq K_{2}=\alpha^{-1} K_{1}+M_{1}^{2} .
$$

o que conclui a prova de (1.36).

Lema 1.1.ii: Se $|-\Delta \xi(0)|<\infty$, existem constantes $K_{3}, K_{4}$ tal que $\forall \alpha>0$,:

$$
\begin{aligned}
\liminf _{\epsilon \rightarrow 0^{+}}\left|\xi_{t}(\epsilon)\right| & \leq K_{3}|-\Delta \xi(0)|+|F|, \\
e^{-\alpha t} \int_{0}^{t} e^{\alpha \tau}\left|\xi_{t}\right|^{2} d \tau & \leq K_{4}, \quad t>0 .
\end{aligned}
$$




\section{Prova.-}

Tomando o produto escalar de (1.24), (1.25) com respeito a $\xi_{t}$, obtemos a desigualdade:

$$
\begin{aligned}
\left|\xi_{t}\right|^{2} \leq & \mid\left(\vec{V} \cdot \nabla \vec{V}, \vec{V}_{t}|+|\left(k \wedge \vec{V}, \vec{V}_{t}\right)\left|+\nu_{0}\right|\left(-\Delta \vec{V}, \vec{V}_{t}\right)\left|+\kappa_{0}\right|\left(-\Delta z, z_{t}\right) \mid+\right. \\
& +\left|\left(\nabla z, \vec{V}_{t}\right)\right|+\left|\left(\nabla \cdot\left(\left(g_{0}+z\right) \vec{V}\right), \xi_{t}\right)\right|+\left|\left(F, z_{t}\right)\right| .
\end{aligned}
$$

Agora por E2 e a estimativa $|\vec{V}| \leq \lambda_{1}^{-1}|-\Delta \vec{V}|$ temos,

a.- $\left|\left(\vec{V} \cdot \nabla \vec{V}, \vec{V}_{t}\right)\right| \leq c_{2}|\vec{V}|^{1 / 2}\|\vec{V}\||-\Delta \vec{V}|^{1 / 2}\left|\xi_{t}\right| \leq c_{2} \lambda_{1}^{-1 / 2} M_{1}|-\Delta \vec{V}|\left|\xi_{t}\right|$,

b.- $\left|\left(k \wedge \vec{V}, \vec{V}_{t}\right)\right| \leq|\vec{V}|\left|\xi_{t}\right| \leq \lambda_{1}^{-1}|-\Delta \vec{V}|\left|\xi_{t}\right| \leq \lambda_{1}^{-1}|-\Delta \xi|\left|\xi_{t}\right|$,

c. $\left|\left(\nabla z, \vec{V}_{t}\right)\right| \leq\|z\|\left|\xi_{t}\right| \leq \lambda_{1}^{-1 / 2}|-\Delta z|\left|\xi_{t}\right| \leq \lambda_{1}^{-1 / 2}|-\Delta \xi|\left|\xi_{t}\right|$,

d.- $g_{0}\left|\left(\nabla \cdot \vec{V}, z_{t}\right)\right| \leq \sqrt{2} g_{0}|| \vec{V} \|\left|\xi_{t}\right| \leq \sqrt{2} g_{0} \lambda_{1}^{-1 / 2}|-\Delta \xi|\left|\xi_{t}\right|$,

e.- $\left.\mid z \nabla \cdot \vec{V}, z_{t}\right)\left.\left|\leq c_{2}\right| z\right|^{1 / 2}|-\Delta z|^{1 / 2}\|\vec{V}\|\left|\xi_{t}\right| \leq c_{2} \lambda_{1}^{-1 / 2} M_{1}|-\Delta \xi|\left|\xi_{t}\right|$

f.- $\left|\left(\vec{V} \cdot \nabla z, z_{t}\right)\right| \leq c_{2}|\vec{V}|^{1 / 2}|\Delta \vec{V}|^{1 / 2}|| z \|\left|\xi_{t}\right| \leq c_{2} M_{1} \lambda_{1}^{-1 / 2}|-\Delta \vec{V}|\left|\xi_{t}\right|$,

g.- $\nu_{0}\left|\left(-\Delta \vec{V}, \vec{V}_{t}\right)\right| \leq \nu_{0}|-\Delta \vec{V}|\left|\xi_{t}\right| \leq \nu_{0}|-\Delta \xi|\left|\xi_{t}\right|$

h.- $\kappa_{0}\left|\left(-\Delta z, z_{t}\right)\right| \leq \kappa_{0}|-\Delta z|\left|\xi_{t}\right| \leq \kappa_{0}|-\Delta \xi|\left|\xi_{t}\right|$,

i.- $\left|\left(F, z_{t}\right)\right| \leq|F|\left|z_{t}\right| \leq|F|\left|\xi_{t}\right|$

Substituindo (a)-(i) em (1.42), obtemos

$$
\left|\xi_{t}\right|^{2} \leq K_{3}|\Delta \xi|\left|\xi_{t}\right|+|F|\left|\xi_{t}\right|
$$


onde:

$$
K_{3}=\lambda_{1}^{-1 / 2}\left(1+3 c_{2} M_{1}+\sqrt{2} g_{0}\right)+\lambda_{1}^{-1}+\nu_{0}+\kappa_{0}
$$

simplificando:

$$
\left|\xi_{t}(t)\right| \leq K_{3}|-\Delta \xi(t)|+|F|, \quad t>0
$$

da qual segue-se a estimativa (1.40):

$$
\liminf _{\epsilon \rightarrow 0^{+}}\left|\xi_{t}(\epsilon)\right| \leq K_{3}|-\Delta \xi(0)|+|F| .
$$

Elevando (1.43) ao quadrado obtemos:

$$
\left|\xi_{t}\right|^{2} \leq 2\left(K_{3}^{2}|-\Delta \xi|^{2}+|F|^{2}\right)
$$

multiplicando por $e^{\alpha t}$ para $\alpha>0$, integrando em $t$ e multiplicando a seguir por $e^{-\alpha t}$, obtemos:

$$
e^{-\alpha t} \int_{0}^{t} e^{\alpha \tau}\left|\xi_{\tau}\right|^{2} d \tau \leq 2 K_{3}^{2} \kappa_{0}^{-1} e^{-\alpha t} \int_{0}^{t} e^{\alpha \tau} \kappa_{0}|-\Delta \xi|^{2} d \tau+2 e^{-\alpha t} \int_{0}^{t} e^{\alpha \tau}|F|^{2} d \tau
$$

aplicando o Lema (1.1.i) temos:

$$
e^{-\alpha t} \int_{0}^{t} e^{\alpha \tau}\left|\xi_{\tau}\right|^{2} d \tau \leq 2 \kappa_{0}^{-1} K_{3}^{2} K_{2}+2 \alpha^{-1}|F|^{2}=K_{4}
$$

provando (1.41).

Lema 1.1.iii: Se $|-\Delta \xi(0)|<\infty$, existe uma constante $M_{3}$ tal que:

$$
\left|\xi_{t}(t)\right| \leq M_{3}, \quad t>0
$$

Prova.-

Diferenciando $(1.24),(1.25)$ com respeito a $t$ e tomando o produto escalar com $\xi_{t}$ obtemos:

$$
\begin{aligned}
\frac{1}{2} \frac{d}{d t}\left|\xi_{t}\right|^{2}+\kappa_{0}\left\|\xi_{t}\right\|^{2} & \leq\left|\left(\vec{V}_{t} \cdot \nabla \vec{V}+\vec{V} \cdot \nabla \vec{V}_{t}, \vec{V}_{t}\right)\right|+\left|\left(\nabla z_{t}, \vec{V}_{t}\right)\right|+ \\
+ & +\left(\nabla \cdot\left(z_{t} \vec{V}+z \vec{V}_{t}\right), z_{t}\right)\left|+g_{0}\right|\left(\nabla \cdot \vec{V}_{t}, z_{t}\right) \mid .
\end{aligned}
$$

Aplicando E1, a limitação dos termos resulta:

a. $\left|\left(\vec{V} \cdot \nabla \vec{V}_{t}, \vec{V}_{t}\right)\right| \leq c_{1}|\vec{V}|^{1 / 2}\|\vec{V}\|^{1 / 2}\left\|\vec{V}_{t}\right\|\left|\vec{V}_{t}\right|^{1 / 2}\left\|\vec{V}_{t}\right\|^{1 / 2} \leq c_{1} \lambda_{1}^{-1 / 4} M_{1}\left|\vec{V}_{t}\right|^{1 / 2}\left\|\vec{V}_{t}\right\|^{3 / 2}$ 


$$
\begin{aligned}
\leq & \frac{\kappa_{0}}{12}\left\|\vec{V}_{t}\right\|^{2}+\frac{9^{3}}{4} \kappa_{0}^{-3} c_{1}^{4} \lambda_{1}^{-1} M_{1}^{4}\left|\vec{V}_{t}\right|^{2} \\
& \text { (pela desigualdade de Young com } p=\frac{4}{3}, q=4, \epsilon=\frac{\kappa_{0}}{9} \text { ) }
\end{aligned}
$$

b. $\left|\left(\vec{V}_{t} \cdot \nabla \vec{V}, \vec{V}_{t}\right)\right| \leq c_{1}\left|\vec{V}_{t}\right|\left\|\vec{V}_{t}\right\|\|\vec{V}\| \leq c_{1} M_{1}\left|\vec{V}_{t}\right|\left\|\vec{V}_{t}\right\| \leq \frac{\kappa_{0}}{12}\left\|\vec{V}_{t}\right\|^{2}+3 \kappa_{0}^{-1} c_{1}^{2} M_{1}^{2}\left|\vec{V}_{t}\right|^{2}$, (pela desigualdade de Young com $p=q=2, \epsilon=\kappa_{0} / 6$ )

c. $\quad\left|\left(\nabla z_{t}, \vec{V}_{t}\right)\right| \leq\left\|z_{t}\right\|\left|\vec{V}_{t}\right| \leq\left\|z_{t}\right\|\left|\xi_{t}\right| \leq \frac{\kappa_{0}}{12}\left\|z_{t}\right\|^{2}+3 \kappa_{0}^{-1}\left|\xi_{t}\right|^{2}$ (pela desigualdade de Young com $p=q=2, \epsilon=\kappa_{0} / 6$ )

d. $\left|\left(z_{t} \nabla \cdot \vec{V}, z_{t}\right)\right| \leq c_{1}\left|z_{t}\right|\|\vec{V}\|\left\|z_{t}\right\| \leq c_{1} M_{1}\left|z_{t}\right|\left\|z_{t}\right\| \leq \frac{\kappa_{0}}{12}\left\|z_{t}\right\|^{2}+3 \kappa_{0}^{-1} c_{1}^{2} M_{1}^{2}\left|z_{t}\right|^{2}$, (pela desigualdade de Young com $p=q=2, \epsilon=\kappa_{0} / 6$ )

e. $\left|\left(\vec{V} \cdot \nabla z_{t}, z_{t}\right)\right| \leq c_{1}|\vec{V}|^{1 / 2}\|\vec{V}\|^{1 / 2}\left\|z_{t}\right\|^{3 / 2}\left|z_{t}\right|^{1 / 2} \leq c_{1} \lambda_{1}^{-1 / 4} M_{1}\left|z_{t}\right|\left\|z_{t}\right\|^{3 / 2}$

$$
\leq \frac{\kappa_{0}}{12}\left\|z_{t}\right\|^{2}+\frac{9^{3}}{4} \kappa_{0}^{-3} c_{1}^{4} \lambda_{1}^{-1} M_{1}^{4}\left|z_{t}\right|^{2}
$$

(pela desigualdade de Young com $p=4 / 3, q=4, \quad \epsilon=\kappa_{0} / 9$ )

f. $\left|\left(z \nabla \cdot \vec{V}_{t}, z_{t}\right)\right| \leq c_{1}|z|^{1 / 2}\|z\|^{1 / 2}\left\|\vec{V}_{t}\right\|\left\|z_{t}\right\|^{1 / 2}\left|z_{t}\right|^{1 / 2} \leq c_{1} \lambda_{1}^{-1 / 4} M_{1}\left|\xi_{t}\right|^{1 / 2}\left\|\xi_{t}\right\|^{3 / 2}$

$$
\leq \frac{\kappa_{0}}{8}\left\|\xi_{t}\right\|^{2}+54 \kappa_{0}^{-3} c_{1}^{4} \lambda_{1}^{-1} M_{1}^{4}\left|\xi_{t}\right|^{2}
$$

(pela desigualdade de Young $\operatorname{com} p=4 / 3, q=4, \epsilon=\kappa_{0} / 6$ )

g. $\left|\left(\vec{V}_{t} \cdot \nabla z, z_{t}\right)\right| \leq c_{1}\left|\vec{V}_{t}\right|^{1 / 2}\left\|\vec{V}_{t}\right\|^{1 / 2}\|z\|\left|z_{t}\right|^{1 / 2}\left\|z_{t}\right\|^{1 / 2} \leq c_{1} M_{1}\left|\xi_{t}\right|\left\|\xi_{t}\right\|$

$$
\leq \frac{\kappa_{0}}{8}\left\|\xi_{t}\right\|^{2}+4 \kappa_{0}^{-1} c_{1}^{2} M_{1}^{2}\left|\xi_{t}\right|^{2}
$$

(pela desigualdade de Young com $p=q=2, \quad \epsilon=\kappa_{0} / 4$ )

h. $g_{0}\left|\left(\nabla \cdot \vec{V}_{t}, z_{t}\right)\right| \leq \sqrt{2} g_{0}\left\|\vec{V}_{t}\right\|\left|\xi_{t}\right| \leq \frac{\kappa_{0}}{12}\left\|\vec{V}_{t}\right\|^{2}+6 \kappa_{0}^{-1} g_{0}^{2}\left|\xi_{t}\right|^{2}$

$$
\text { (pela desigualdade de Young com } p=q=2, \epsilon=\kappa_{0} / 6 \text { ) }
$$


Substituindo (a)-(h) em (1.45) obtém-se:

$$
\frac{1}{2} \frac{d}{d t}\left|\xi_{t}\right|^{2}+\kappa_{0}\left\|\xi_{t}\right\|^{2} \leq \frac{\kappa_{0}}{4}\left(\left\|\vec{V}_{t}\right\|^{2}+\left\|z_{t}\right\|^{2}\right)+\frac{\kappa_{0}}{4}\left\|\xi_{t}\right\|^{2}+\frac{1}{2} K_{5}\left|\xi_{t}\right|^{2} \quad \text { e }
$$

simplificando obtemos

$$
\frac{d}{d t}\left|\xi_{t}\right|^{2}+\kappa_{0}\left\|\xi_{t}\right\|^{2} \leq K_{5}\left|\xi_{t}\right|^{2}
$$

onde

$$
K_{5}=\frac{945}{2} \kappa_{0}^{-3} c_{1}^{4} \lambda_{1}^{-1} M_{1}^{4}+14 \kappa_{0}^{-1} c_{1}^{2} M_{1}^{2}+6 \kappa_{0}^{-1}+12 \kappa_{0}^{-1} g_{0}^{2} .
$$

Multiplicando (1.46) por $e^{\alpha t}$ para algum $\alpha>0$ :

$$
\frac{d}{d t}\left(e^{\alpha t}\left|\xi_{t}\right|^{2}\right)+\kappa_{0} e^{\alpha t}\left\|\xi_{t}\right\|^{2} \leq K_{5} e^{\alpha t}\left|\xi_{t}\right|^{2}+\alpha e^{\alpha t}\left|\xi_{t}\right|^{2}
$$

Integrando com respeito a $t$, e multiplicando a seguir por $e^{-\alpha t}$ resulta:

$$
\begin{aligned}
\left|\xi_{t}\right|^{2}+\kappa_{0} e^{-\alpha t} \int_{\epsilon}^{t} e^{\alpha \tau}\left\|\xi_{\tau}\right\|^{2} d \tau \leq & e^{-\alpha t}\left|\xi_{t}(\epsilon)\right|^{2}+e^{-\alpha t}\left(K_{5}+\alpha\right) \int_{\epsilon}^{t} e^{\alpha \tau}\left|\xi_{\tau}\right|^{2} d \tau \\
& \text { Tomando } \limsup _{\epsilon \rightarrow 0^{+}}: \\
\leq & e^{-\alpha t} \limsup _{\epsilon \rightarrow 0^{+}}\left|\xi_{t}(\epsilon)\right|^{2}+\left(K_{5}+\alpha\right) K_{4}, \quad(\text { por }(1.41)) \\
\leq & 2 K_{3}^{2}|-\Delta \xi(0)|^{2}+\left(K_{5}+\alpha\right) K_{4}+2|F|^{2}=M_{3}^{2}, \quad(1.47
\end{aligned}
$$

onde na ultima desigualdade temos usado (1.40), o que conclui a prova de (1.44)

Lema 1.1.iv: Se $|-\Delta \xi(0)|<\infty$, existe constante $M_{2}$ tal que:

$$
|-\Delta \xi(t)| \leq M_{2}, \quad t>0
$$

Prova.-

Tomando o produto escalar de $((1.24),-\Delta \vec{V})+((1.25),-\Delta z)$ vemos que

$$
\begin{aligned}
\kappa_{0}|-\Delta \xi|^{2} \leq & |(\vec{V} \cdot \nabla \vec{V},-\Delta \vec{V})|+|(\nabla z,-\Delta \vec{V})|+\left|\left(\nabla \cdot\left(\left(g_{0}+z\right) \vec{V}\right),-\Delta z\right)\right|+ \\
& +\left|\left(\vec{V}_{t},-\Delta \vec{V}\right)\right|+|(F,-\Delta z)|
\end{aligned}
$$

Os termos serão estimados usando E2: 


$$
\text { a.- } \begin{aligned}
|(\vec{V} \cdot \nabla \vec{V},-\Delta \vec{V})| & \leq c_{2}|\vec{V}|^{1 / 2}|| \vec{V}||-\left.\Delta \vec{V}\right|^{3 / 2} \leq c_{2} \lambda_{1}^{-1 / 4} M_{1}^{3 / 2}|-\Delta \xi|^{1 / 2}|-\Delta \vec{V}| \\
\leq & \frac{\kappa_{0}}{2}|-\Delta \vec{V}|^{2}+\frac{1}{2} c_{2}^{4} \kappa_{0}^{-1} \lambda_{1}^{-1 / 2} M_{1}^{3}|-\Delta \xi|
\end{aligned}
$$

(pela desigualdade de Young, com $p=q=2, \epsilon=\kappa_{0}$ )

b.- $|(\nabla z,-\Delta \vec{V})| \leq\|z\||-\Delta \vec{V}| \leq M_{1}|-\Delta \xi|$

c. $-g_{0}|\nabla \cdot \vec{V},-\Delta z| \leq \sqrt{2} g_{0}|| \vec{V} \||-\Delta z| \leq \sqrt{2} g_{0} M_{1}|-\Delta \xi|$

d.- $|(\vec{V} \cdot \nabla z,-\Delta z)| \leq c_{2}|\vec{V}|^{1 / 2}|-\Delta \vec{V}|^{1 / 2}|| z|||-\Delta z| \leq c_{2} \lambda_{1}^{-1 / 4} M_{1}^{3 / 2}|-\Delta \xi|^{1 / 2}|-\Delta z|$

$$
\leq \frac{\kappa_{0}}{4}|-\Delta z|^{2}+\kappa_{0}^{-1} c_{2}^{2} \lambda_{1}^{-1 / 2} M_{1}^{3}|-\Delta \xi|
$$

(pela desigualdade de Young, com: $p=q=2, \epsilon=\kappa_{0} / 2$ )

$$
\text { e.- } \begin{aligned}
|(z \nabla \cdot \vec{V} .-\Delta z)| & \leq c_{2}|z|^{1 / 2}|\vec{V} \||-\left.\Delta z\right|^{3 / 2} \leq c_{2} \lambda_{1}^{-1 / 4} M_{1}^{3 / 2}|-\Delta \xi||-\Delta z| \\
& \leq \frac{\kappa_{0}}{4}|-\Delta z|^{2}+2 \kappa_{0}^{-1} c_{2}^{2} \lambda_{1}^{-1 / 2} M_{1}^{3}|-\Delta \xi|
\end{aligned}
$$

(pela desigualdade de Young, com: $p=q=2, \epsilon=\kappa_{0} / 2$ )

f.- $|(F,-\Delta z)| \leq|F||-\Delta z| \leq|F||-\Delta \xi|$

g.- $\left|\left(\vec{V}_{t},-\Delta \vec{V}\right)\right| \leq\left|\vec{V}_{t}\right||-\Delta \vec{V}| \leq\left|\xi_{t}\right||-\Delta \xi| \leq M_{3}|-\Delta \xi|$, (pelo Lema 1.1.iii)

Substituindo (a)-(g) em (1.49) obtemos:

$$
\kappa_{0}|-\Delta \xi|^{2} \leq \frac{\kappa_{0}}{2}\left(|-\Delta \vec{V}|^{2}+|-\Delta z|^{2}\right)+\frac{1}{2} M_{2}|-\Delta \xi|
$$

Simplificando tem-se: $\quad \frac{1}{2} \kappa_{0}|-\Delta \xi|^{2} \leq \frac{1}{2} M_{2}\left|\xi_{t}\right|, \quad$ onde:

$$
M_{2}=5 \kappa_{0}^{-1} c_{2}^{4} \lambda_{1}^{-1 / 2} M_{1}^{3}+2\left(1+\sqrt{2} g_{0}\right) M_{1}+2|F|+2 M_{3}
$$

e portanto $|-\Delta \xi| \leq M_{2}$, o que completa a prova de (1.48) e também do Lema (1.1). 


\subsubsection{Estimativa para $(-\Delta)^{3 / 2} \xi$ em $\mathbb{H} \times H$}

A seguinte estimativa técnica preliminar será necessária para mostrar que $\left\|\xi_{t}\right\| \leq M_{5}, \quad \forall 0<t \leq \tau_{0}$, (isto é, para a parte transiente do sistema).

Lema 1.2 Suponha que as condições $\left|(-\Delta)^{3 / 2} \xi(0)\right|<\infty,|-\Delta F|<\infty$, e (1.35) sejam satisfeitas. Sejam

$$
\begin{aligned}
\tau_{0} & =\left[2\left(1+\kappa_{0}^{-1}+3 \kappa_{0}^{-1} c_{5}^{2} \lambda_{1}^{-1} M_{2}^{2}\right)\right]^{-1} e \\
M_{4} & =\left|(-\Delta)^{3 / 2} \xi(0)\right|^{2}+\kappa_{0}^{-1}|-\Delta F|^{2} \tau_{0} .
\end{aligned}
$$

Então

$$
\left|(-\Delta)^{3 / 2} \xi(t)\right|^{2} \leq e M_{4}, \quad \text { para } 0<t \leq \tau_{0} .
$$

onde e é a base do logaritmo natural.

\section{$\underline{\text { Prova }}$}

Tomando o produto escalar de $(1.24),(1.25) \operatorname{com}(-\Delta)^{3} \xi$ vemos que

$$
\begin{aligned}
\frac{1}{2} \frac{d}{d t}\left|(-\Delta)^{3 / 2} \xi\right|^{2} & +\kappa_{0}\left|(-\Delta)^{2} \xi\right|^{2} \leq\left|(-\Delta)^{3 / 2} \vec{V}\right|^{2}+\left|(-\Delta)^{3 / 2} z\right|\left|(-\Delta)^{2} \vec{V}\right|+|\Delta F|\left|(-\Delta)^{2} z\right| \\
& +\left|\left(-\Delta(\vec{V} \cdot \nabla \vec{V}),(-\Delta)^{2} \vec{V}\right)\right|+\left|\left(-\Delta \nabla \cdot\left(\left(g_{0}+z\right) \vec{V}\right),(-\Delta)^{2} z\right)\right|
\end{aligned}
$$

Usando E5, o Lema (1.1), e a desigualdade de Young com $p=q=2, \epsilon=\kappa_{0} / 2$, os termos são estimados como segue:

$$
\begin{aligned}
& \text { a.- }\left|(-\Delta)^{3 / 2} z\right|\left|(-\Delta)^{2} \vec{V}\right| \leq\left|(-\Delta)^{3 / 2} \xi\right|\left|(-\Delta)^{2} \vec{V}\right| \leq \frac{\kappa_{0}}{4}\left|(-\Delta)^{2} \vec{V}\right|^{2}+\kappa_{0}^{-1}\left|(-\Delta)^{3 / 2} \xi\right|^{2} \\
& \text { b.- }|-\Delta F|\left|(-\Delta)^{2} z\right| \leq \frac{\kappa_{0}}{4}\left|(-\Delta)^{2} z\right|^{2}+\kappa_{0}^{-1}|-\Delta F|^{2} \\
& \text { c. }\left|\left(-\Delta(\vec{V} \cdot \nabla \vec{V}),(-\Delta)^{2} \vec{V}\right)\right| \leq \frac{c_{5}}{\lambda_{1}^{1 / 2}}|-\Delta \vec{V}|\left|(-\Delta)^{3 / 2} \vec{V}\right|\left|(-\Delta)^{2} \vec{V}\right| \\
& \leq c_{5} \lambda_{1}^{-1 / 2} M_{2}\left|(-\Delta)^{3 / 2} \xi\right|\left|(-\Delta)^{2} \vec{V}\right| \\
& \leq \frac{\kappa_{0}}{4}\left|(-\Delta)^{2} \vec{V}\right|^{2}+\kappa_{0}^{-1} c_{5}^{2} \lambda_{1}^{-1} M_{2}^{2}|-\Delta \xi|^{2}
\end{aligned}
$$




$$
\begin{aligned}
\text { d.- }\left|\left(-\Delta \nabla \cdot\left(\left(g_{0}+z\right) \vec{V}\right),(-\Delta)^{2} z\right)\right| \leq & \left|-\Delta\left(\left(g_{0}+z\right) \nabla \cdot \vec{V}\right)\right|\left|(-\Delta)^{2} z\right|+ \\
& +\left|-\Delta\left(\vec{V} \cdot \nabla\left(g_{0}+z\right)\right)\right|\left|(-\Delta)^{2} z\right| \\
\leq & c_{5} \lambda_{1}^{-1 / 2}|-\Delta z|\left|(-\Delta)^{3 / 2} \vec{V}\right|\left|\Delta^{2} z\right|+ \\
& +c_{5} \lambda_{1}^{-1 / 2}|-\Delta \vec{V}|\left|(-\Delta)^{3 / 2} z\right|\left|(-\Delta)^{2} z\right| \\
\leq & 2 c_{5} \lambda_{1}^{-1 / 2} M_{2}\left|(-\Delta)^{3 / 2} \xi\right|\left|\Delta^{2} z\right| \\
\leq & \frac{\kappa_{0}}{4}\left|(-\Delta)^{2} z\right|^{2}+4 \kappa_{0}^{-1} c_{5}^{2} \lambda_{1}^{-1} M_{2}^{2}\left|(-\Delta)^{3 / 2} \xi\right|^{2}
\end{aligned}
$$

Substituindo (a)-(d) em (1.51) obtém-se:

$$
\begin{aligned}
\frac{1}{2} \frac{d}{d t}\left|(-\Delta)^{3 / 2} \xi\right|^{2}+\kappa_{0}\left|(-\Delta)^{2} \xi\right|^{2} & \leq \frac{\kappa_{0}}{2}\left(\left|(-\Delta)^{2} \vec{V}\right|^{2}+\left|(-\Delta)^{2} z\right|^{2}\right)+ \\
+ & \left(1+\kappa_{0}^{-1}+3 \kappa_{0}^{-1} c_{5}^{2} \lambda_{1}^{-1} M_{2}^{2}\right)\left|(-\Delta)^{3 / 2} \xi\right|^{2}+\kappa_{0}^{-1}|-\Delta F|^{2}
\end{aligned}
$$

Simplificando os termos, resulta:

$$
\left|(-\Delta)^{3 / 2} \xi\right|^{2}+\kappa_{0}\left|(-\Delta)^{2} \xi\right|^{2} \leq 2\left(1+\kappa_{0}^{-1}+3 \kappa_{0}^{-1} c_{5}^{2} \lambda_{1}^{-1} M_{2}^{2}\right)\left|(-\Delta)^{3 / 2} \xi\right|^{2}+2 \kappa_{0}^{-1}|-\Delta F|^{2}
$$

Fazendo,

$$
\begin{aligned}
X & =\left|(-\Delta)^{3 / 2} \xi(t)\right|^{2} \\
a & =2\left(1+\kappa_{0}^{-1}+3 \kappa_{0}^{-1} c_{5}^{2} \lambda_{1}^{-1} M_{2}^{2}\right) \\
b & =2 \kappa_{0}^{-1}|-\Delta F|^{2}
\end{aligned}
$$

temos:

$$
\frac{d X}{d t} \leq a X+b
$$

Aplicando o Lema clássico de Gronwall com, $g=a, h=b, \quad$ constantes, obtém-se após simplificações:

$$
X \leq\left(X_{0}+b a^{-1}\right) e^{a t}
$$

ou

$$
\left|(-\Delta)^{3 / 2} \xi(t)\right|^{2} \leq e M_{4}, \quad \text { para } \quad 0<t \leq a^{-1}=\tau_{0} .
$$

onde, $\quad M_{4}=\left|(-\Delta)^{3 / 2} \xi(0)\right|^{2}+b a^{-1}$, o que conclui a prova do Lema (1.2). 


\subsubsection{Estimativa para $\xi_{t}$ em $\mathbb{V} \times V$}

A terceira de nossas estimativas básicas afirma o seguinte:

Lema 1.3 Se $\left|(-\Delta)^{3 / 2} \xi(0)\right|<\infty, \quad|-\Delta F|<\infty$ e $\|\xi(t)\| \leq M_{1}, \quad \forall t \in[0, \infty)$, então existe uma constante $M_{5}$, tal que

$$
\left\|\xi_{t}(t)\right\| \leq M_{5}, \quad t>0
$$

No que segue, como em [31], seja $\tau(t)=\min \left(t, \tau_{0}\right) \quad\left(\tau_{0}\right.$ como no Lema (1.2)) e $\sigma(t)=$ $\tau(t) e^{\alpha t}, \alpha>0$.

Prova.- A prova será feita em duas etapas:

i) Existe uma constante $K_{8}$ tal que

$$
\left\|\xi_{t}(t)\right\| \leq K_{8}, \quad \forall t \geq \tau_{0}
$$

onde $\tau_{0}$ é dado como no Lema (1.2).

\section{Prova.-}

Fazendo $\left((1.24)_{t},-\Delta \vec{V}_{t}\right)+\left((1.25)_{t},-\Delta z_{t}\right)$, temos

$$
\begin{aligned}
\frac{1}{2} \frac{d}{d t}\left\|\xi_{t}\right\|^{2}+\kappa_{0}\left|-\Delta \xi_{t}\right|^{2} & \leq\left|\left(\vec{V}_{t} \cdot \nabla \vec{V}+\vec{V} \cdot \nabla \vec{V}_{t},-\Delta \vec{V}_{t}\right)\right|+\left|\left(\nabla z_{t},-\Delta \vec{V}_{t}\right)\right|+ \\
+ & \left.\mid \nabla \cdot\left(z_{t} \vec{V}+z \vec{V}_{t}\right),-\Delta z_{t}\right)\left|+g_{0}\right|\left(\nabla \cdot \vec{V}_{t},-\Delta z_{t}\right) \mid .
\end{aligned}
$$

Os termos em ( 1.53 ) são estimados usando $E_{2}$ :

$$
\begin{aligned}
\left|\left(\vec{V}_{t} \cdot \nabla \vec{V},-\Delta \vec{V}_{t}\right)\right| & \leq c_{2}\left|\vec{V}_{t}\right|^{1 / 2}\left|-\Delta \vec{V}_{t}\right|^{1 / 2}\|\vec{V}\|\left|-\Delta \vec{V}_{t}\right| \leq c_{2} M_{1} \lambda_{1}^{-1 / 4}\left\|\xi_{t}\right\|^{1 / 2}\left|-\Delta \vec{V}_{t}\right|^{3 / 2} \\
& \leq \frac{\kappa_{0}}{12}\left|-\Delta \vec{V}_{t}\right|^{2}+\frac{9^{3}}{4} \kappa_{0}^{-3} c_{2}^{4} M_{1}^{4} \lambda_{1}^{-1}\left\|\xi_{t}\right\|^{2} \\
& \text { ( pela desigualdade de Young com: } \left.p=4 / 3, q=4 \epsilon=\kappa_{0} / 9\right) \\
\left|\left(\vec{V} \cdot \nabla \vec{V}_{t},-\Delta \vec{V}_{t}\right)\right| & \leq c_{2}|\vec{V}|^{1 / 2}|-\Delta \vec{V}|^{1 / 2}|| \vec{V}_{t}\left\|\left|-\Delta \vec{V}_{t}\right| \leq c_{2} M_{1}^{1 / 2} \lambda_{1}^{-1 / 4} M_{2}^{1 / 2}\right\| \xi_{t} \|\left|-\Delta \vec{V}_{t}\right| \\
& \leq \frac{\kappa_{0}}{12}\left|-\Delta \vec{V}_{t}\right|^{2}+3 \kappa_{0}^{-1} c_{2}^{2} M_{1} M_{2} \lambda_{1}^{-1 / 2}\left\|\xi_{t}\right\|^{2} \\
& \left(\text { pela desigualdade de Young com: } p=q=2 \epsilon=\kappa_{0} / 6\right)
\end{aligned}
$$




$$
\left|\left(\nabla z_{t},-\Delta \vec{V}_{t}\right)\right| \leq|| z_{t}\left\|\left|-\Delta \vec{V}_{t}\right| \leq\right\| \xi_{t}\left\|\left|-\Delta \vec{V}_{t}\right| \leq \frac{\kappa_{0}}{12}\left|-\Delta \vec{V}_{t}\right|^{2}+3 \kappa_{0}^{-1}\right\| \xi_{t} \|^{2}
$$

( pela desigualdade de Young com: $p=q=2 \epsilon=\kappa_{0} / 6$ )

$$
\begin{aligned}
\left|\left(z_{t} \cdot \nabla \vec{V},-\Delta z_{t}\right)\right| & \leq c_{2}\left|z_{t}\right|^{1 / 2}\left|-\Delta z_{t}\right|^{1 / 2}\|\vec{V}\|\left|-\Delta z_{t}\right| \leq c_{2} M_{1} \lambda_{1}^{-1 / 4}\left\|\xi_{t}\right\|^{1 / 2}\left|-\Delta z_{t}\right|^{3 / 2} \\
& \leq \frac{\kappa_{0}}{16}\left|-\Delta z_{t}\right|^{2}+\frac{12^{3}}{4} \kappa_{0}^{-3} c_{2}^{4} M_{1}^{4} \lambda_{1}^{-1}\left\|\xi_{t}\right\|^{2}
\end{aligned}
$$

( pela desigualdade de Young com: $p=4 / 3, q=4 \epsilon=\kappa_{0} / 12$ )

$$
\begin{aligned}
\left|\left(\vec{V} \cdot \nabla z_{t},-\Delta z_{t}\right)\right| & \leq c_{2}|\vec{V}|^{1 / 2}|-\Delta \vec{V}|^{1 / 2}\left\|z_{t}\right\|\left|-\Delta z_{t}\right| \leq c_{2} M_{1}^{1 / 2} \lambda_{1}^{-1 / 4} M_{2}^{1 / 2}\left\|\xi_{t}\right\|\left|-\Delta z_{t}\right| \\
& \leq \frac{\kappa_{0}}{16}\left|-\Delta z_{t}\right|^{2}+4 \kappa_{0}^{-1} c_{2}^{2} M_{1} M_{2} \lambda_{1}^{-1 / 2}\left\|\xi_{t}\right\|^{2}
\end{aligned}
$$

( pela desigualdade de Young com: $p=q=2 \epsilon=\kappa_{0} / 8$ )

$$
\begin{aligned}
\left|\left(z \cdot \nabla \vec{V}_{t},-\Delta z_{t}\right)\right| & \leq c_{2}|z|^{1 / 2}|-\Delta z|^{1 / 2}|| \vec{V}_{t}\left\|\left|-\Delta z_{t}\right| \leq c_{2} M_{1}^{1 / 2} \lambda_{1}^{-1 / 4} M_{2}^{1 / 2}\right\| \xi_{t} \|\left|-\Delta z_{t}\right| \\
& \leq \frac{\kappa_{0}}{16}\left|-\Delta z_{t}\right|^{2}+4 \kappa_{0}^{-1} c_{2}^{2} M_{1} M_{2} \lambda_{1}^{-1 / 2}\left\|\xi_{t}\right\|^{2}
\end{aligned}
$$

( pela desigualdade de Young com: $p=q=2 \epsilon=\kappa_{0} / 8$ )

$$
\begin{aligned}
\left|\left(\vec{V}_{t} \cdot \nabla z,-\Delta z_{t}\right)\right| & \leq c_{2}\left|\vec{V}_{t}\right|^{1 / 2}\left|-\Delta \vec{V}_{t}\right|^{1 / 2}\|z\|\left|-\Delta z_{t}\right| \leq c_{2} M_{1} \lambda_{1}^{-1 / 4}\left\|\xi_{t}\right\|^{1 / 2}\left|-\Delta \xi_{t}\right|^{3 / 2} \\
& \leq \frac{\kappa_{0}}{4}\left|-\Delta \xi_{t}\right|^{2}+\frac{27}{4} \kappa_{0}^{-3} c_{2}^{4} M_{1}^{4} \lambda_{1}^{-1}\left\|\xi_{t}\right\|^{2}
\end{aligned}
$$

( pela desigualdade de Young com: $p=4 / 3, q=4 \epsilon=\kappa_{0} / 3$ )

$$
\begin{aligned}
g_{0}\left|\left(\nabla \cdot \vec{V}_{t},-\Delta \vec{V}_{t}\right)\right| & \leq \frac{3}{2} g_{0}\left\|\vec{V}_{t}\right\|\left|-\Delta z_{t}\right| \leq \frac{3}{2} g_{0}\left\|\xi_{t}\right\|\left|-\Delta z_{t}\right| \\
& \leq \frac{\kappa_{0}}{16}\left|-\Delta z_{t}\right|^{2}+18 \kappa_{0}^{-1} g_{0}^{2}\left\|\xi_{t}\right\|^{2}
\end{aligned}
$$

( pela desigualdade de Young com: $p=q=2 \epsilon=\kappa_{0} / 8$ )

Substituindo estas estimativas em ( 1.53) tem-se:

$$
\frac{1}{2} \frac{d}{d t}\left\|\xi_{t}\right\|^{2}+\kappa_{0}\left|-\Delta \xi_{t}\right|^{2} \leq \frac{\kappa_{0}}{4}\left(\left|-\Delta \vec{V}_{t}\right|^{2}+\left|-\Delta z_{t}\right|^{2}+\left|\Delta \xi_{t}\right|^{2}\right)+\frac{1}{2} K_{8}\left\|\xi_{t}\right\|^{2}
$$


onde:

$$
K_{8}=1242 \kappa_{0}^{-3} c_{2}^{4} \lambda_{1}^{-1} M_{1}^{4}+22 \kappa_{0}^{-1} c_{2}^{2} \lambda_{1}^{-1 / 2} M_{1} M_{2}+8 \kappa_{0}^{-1}+36 \kappa_{0}^{-1} g_{0}^{2}
$$

Simplificando, resulta

$$
\frac{d}{d t}\left\|\xi_{t}\right\|^{2}+\kappa_{0}\left|-\Delta \xi_{t}\right|^{2} \leq K_{8}\left\|\xi_{t}\right\|^{2}
$$

A estimativa (1.47) implica na existência de uma seqüência $\epsilon_{n} \rightarrow 0^{+}$tal que

$$
\epsilon_{n}\left\|\xi_{t}\left(\epsilon_{n}\right)\right\|^{2} \rightarrow 0
$$

Multiplicando ( 1.54$)$ por $\sigma(t)$ :

$$
\frac{d}{d t}\left(\sigma(t)\left\|\xi_{t}\right\|^{2}\right)+\kappa_{0} \sigma(t)\left|-\Delta \xi_{t}\right|^{2} \leq K_{8} \sigma(t)\left\|\xi_{t}\right\|^{2}+\left(1+\alpha \tau_{0}\right) e^{\alpha t}\left\|\xi_{t}\right\|^{2}
$$

e integrando de $\epsilon_{n}$ a $t$, vemos que

$$
\begin{aligned}
\sigma(t)\left\|\xi_{t}\right\|^{2}+\kappa_{0} \int_{\epsilon_{n}}^{t} \sigma(\tau)\left|-\Delta \xi_{\tau}\right|^{2} d \tau \leq & \left(K_{8} \tau_{0}+1+\alpha \tau_{0}\right) \int_{\epsilon}^{t} e^{\alpha \tau}\left\|\xi_{\tau}\right\|^{2} d \tau \\
& +\sigma\left(\epsilon_{n}\right)\left\|\xi_{t}\left(\epsilon_{n}\right)\right\|^{2}
\end{aligned}
$$

Tomando $\epsilon_{n} \rightarrow 0^{+}$, e multiplicando por $e^{-\alpha t}$ para $\alpha>0$ :

$$
\begin{aligned}
e^{-\alpha t} \sigma(t)\left\|\xi_{t}\right\|^{2} & \leq\left(K_{8} \tau_{0}+1+\alpha \tau_{0}\right) e^{-\alpha t} \int_{0}^{t} e^{\alpha \tau}\left\|\xi_{\tau}\right\|^{2} d \tau \\
& \leq \kappa_{0}^{-1}\left(K_{8} \tau_{0}+1+\alpha \tau_{0}\right) M_{3}^{2} \quad(\text { por }(1.47))
\end{aligned}
$$

Alem disso, $\quad e^{-\alpha t} \sigma(t)=\tau(t)=\min \left\{t, \tau_{0}\right\}=\tau_{0}, \quad$ para $t \geq \tau_{0}$. Substituindo este resultado na ultima estimativa obtemos:

$$
\left\|\xi_{t}\right\|^{2} \leq \kappa_{0}^{-1}\left(K_{8}+\tau_{0}^{-1}+\alpha\right) M_{3}^{2}=K_{9}, \quad \text { para } t \geq \tau_{0}
$$

provando (i).

ii)

$$
\left\|\xi_{t}(t)\right\| \leq K_{11}, \quad 0<t \leq \tau_{0}
$$

onde $K_{11}$ é uma constante definida ao longo da prova. 


\section{Prova.-}

Multiplicando (1.24) e (1.25) por $(-\Delta)^{1 / 2}$ pode-se estimar (1.55) diretamente, usando $E_{4}$, os Lemas $(1.1),(1.2)$ e considerando $0<t \leq \tau_{0}$ :

$$
\begin{aligned}
\left\|\vec{V}_{t}\right\| \leq & \left|(-\Delta)^{1 / 2} \vec{V} \cdot \nabla \vec{V}\right|+\nu_{0}\left\|(-\Delta)^{3 / 2} \vec{V}\right\|+\left|(-\Delta)^{1 / 2}(k \wedge \vec{V})\right|+|-\Delta z| \\
\leq & c_{4} \lambda_{1}^{-1} M_{2}^{2}+\nu_{0} \sqrt{e} M_{4}^{1 / 2}+M_{1}+M_{2}=K_{10} \\
\left\|z_{t}\right\| \leq & \kappa_{0}\left|(-\Delta)^{3 / 2} z\right|+\left|(-\Delta)^{1 / 2} \nabla \cdot\left(g_{0}+z\right) \vec{V}\right|+\left|(-\Delta)^{1 / 2} F\right| \\
\leq & \kappa_{0}\left|(-\Delta)^{3 / 2} z\right|+\left|(-\Delta)^{1 / 2} \nabla\left(g_{0}+z\right) \nabla \cdot \vec{V}\right|+\left|(-\Delta)^{1 / 2} \vec{V} \cdot \nabla\left(g_{0}+z\right)\right|+ \\
& +\left|(-\Delta)^{1 / 2} F\right| \\
\leq & \kappa_{0} \sqrt{e} M_{4}^{1 / 2}+2 c_{4} \lambda_{1}^{-1 / 2}|-\Delta z||\Delta \vec{V}|+\left|(-\Delta)^{1 / 2} F\right| \\
\leq & \kappa_{0} \sqrt{e} M_{4}^{1 / 2}+c_{4} \lambda_{1}^{-1 / 2} M_{2}^{2}+\left|(-\Delta)^{1 / 2} F\right|=K_{10}^{\prime}
\end{aligned}
$$

Estas duas ultimas estimativas levam a

$$
\left\|\xi_{t}\right\|=\left(\left\|\vec{V}_{t}\right\|^{2}+\left\|z_{t}\right\|^{2}\right) \leq\left(K_{10}^{2}+K_{10^{\prime}}^{2}\right)=K_{11}, \quad 0<t \leq \tau_{0}
$$

concluindo a demonstração do Lema (1.3).

Observamos que outra demonstração destes resultados pode ser derivada de aproximações usuais de Faedo - Galerkin, juntamente com argumentos de limites. É importante observar que as estimativas usuais de Galerkin uniforme se cumprem geralmente com limitações exponenciais com respeito ao número de Reynolds envolvidos e os dados iniciais. As estimativas que aqui obtivemos são polinômios em $\kappa_{0}^{-1}$ e portanto mais precisas. 


\subsection{Construção da Variedade Inercial Aproximada}

Lembremos brevemente algumas notações da teoria das Variedades Inerciais Aproximadas (VIA). Sejam $\xi=\left(\begin{array}{c}\vec{V} \\ z\end{array}\right), \quad \mathbb{M}_{0}=\left(\begin{array}{cc}\nu_{0} & 0 \\ 0 & \kappa_{0}\end{array}\right), \quad f=\left(\begin{array}{c}0 \\ F\end{array}\right), \quad$ então as equações (1.24), (1.25) podem se escrever como:

$$
\begin{aligned}
\frac{d \xi}{d t} & =-\mathbb{M}_{0} A_{0} \xi+G(\xi)+f, \quad t>0, \\
\xi(0) & =\xi_{0} .
\end{aligned}
$$

onde $\quad A_{0}=-\Delta, \quad$ e $\quad G(\xi)=\left(\begin{array}{c}-(\vec{V} \cdot \nabla) \vec{V}-k \wedge \vec{V}-\nabla z \\ -\nabla \cdot\left(\left(g_{0}+z\right) \vec{V}\right)\end{array}\right)$.

Seja $P$ a projeção de $\mathbb{H} \times H$ sobre o espaço gerado pelas primeiras $\mathrm{m}$ - autofunções de $A_{0}$, e denotemos

$$
p=P \xi, \quad q=(I-P) \xi=Q \xi
$$

Temos a seguinte estimativa da relação entre as várias normas para as projeções nos modos baixos e modos altos respectivamente (onde $\lambda_{j}$ é o autovalor associado à j-ésima auto-função de $A_{0}$ )

E6.- $\left\{\begin{array}{l}|\Delta p| \leq \lambda_{m}^{1 / 2}\|p\| \leq \lambda_{m}|p| \\ |q| \leq \lambda_{m}^{-1 / 2}\|q\| \leq \lambda_{m}^{-1}|\Delta q|\end{array}\right.$

Prova.-

Se $p$ e $q$ são decompostas nas componentes de Fourier de $\xi=p+q$ :

$$
p=\sum_{i=1}^{m} \xi_{i} \mathrm{v}_{i}, \quad q=\sum_{i=m+1}^{\infty} \xi_{i} \mathrm{v}_{i}, \quad \text { onde } \quad \xi_{i}=\left(\xi, \mathrm{v}_{i}\right)
$$

isto permite avaliar as normas como:

$$
\begin{aligned}
|p| & =\sqrt{\sum_{i=1}^{m} \xi_{i}^{2}}, \quad\|p\|=\sqrt{\sum_{i=1}^{m} \lambda_{i} \xi_{i}^{2}}, \quad|\Delta p|=\sqrt{\sum_{i=1}^{m} \lambda_{i}^{2} \xi_{i}^{2}} \\
|q| & =\sqrt{\sum_{i=m+1}^{\infty} \xi_{i}^{2}}, \quad\|q\|=\sqrt{\sum_{i=m+1}^{\infty} \lambda_{i} \xi_{i}^{2}}, \quad|\Delta q|=\sqrt{\sum_{i=m+1}^{\infty} \lambda_{i}^{2} \xi_{i}^{2}}
\end{aligned}
$$


Destas caracterizações das normas E6 segue trivialmente.

Usando as projeções $P$ e $Q$ em ( 1.56 ) obtém-se o sistema equivalente de equações

$$
\begin{array}{ll}
\frac{d p}{d t}=-\mathbb{M}_{0} A_{0} p+P G(p+q)+P f, & p(0)=P \xi_{0}, \\
\frac{d q}{d t}=-\mathbb{M}_{0} A_{0} q+Q G(p+q)+Q f, & q(0)=Q \xi_{0} .
\end{array}
$$

Se $q=\Phi(p)=0$, a dinâmica é aproximada pela equação

$$
\frac{d \tilde{p}}{d t}=-\mathbb{M}_{0} A_{0} \tilde{p}+P G(\tilde{p})+P f, \quad \tilde{p}(0)=P \xi_{0},
$$

isto é, um sistema de dimensão finita. Neste caso estamos eliminando a interação entre os modos mais baixos com os modos mais altos, desprezando a sua contribuição para a dinâmica do sistema. Métodos numéricos baseados no truncamento (1.59) são chamados classicamente de métodos de Galerkin.

A aproximação espectral mais simples, usada no chamado método de Galerkin Não Linear para (1.57) - (1.58), é definida através da solução do sistema

$$
\begin{aligned}
\frac{d \bar{p}}{d t} & =-\mathbb{M}_{0} A_{0} \bar{p}+P G(\bar{p}+\Phi(\bar{p}))+P f, \\
\bar{p}(0) & =P \xi_{0}
\end{aligned}
$$

e a VIA é o gráfico da equação

$$
q=\Phi(p)=\mathbb{M}_{0}^{-1} A_{0}^{-1}(Q G(p)+Q f),
$$

definida para $p \in P(\mathbb{H} \times H)$.

Supondo que $\xi=p+q$ é uma solução de (1.56), e $\bar{\xi}=\bar{p}+\bar{q}$ é uma solução de (1.60), definimos:

$$
\begin{aligned}
& \hat{q}=\Phi(p)=\mathbb{M}_{0}^{-1} A_{0}^{-1}(Q G(p)+Q f), \\
& \bar{q}=\Phi(\bar{p})=\mathbb{M}_{0}^{-1} A_{0}^{-1}(Q G(\bar{p})+Q f),
\end{aligned}
$$


Demonstramos a seguinte estimativa:

Lema 1.4 Satisfeitas as hipóteses do Lema (1.3), temos

$$
|-\Delta(q-\hat{q})| \leq \kappa_{0}^{-1} M_{6}\|q\|+\kappa_{0}^{-1}\left|q_{t}\right| .
$$

\section{Prova}

Subtraindo (1.63) de (1.58) obtemos

$$
\mathbb{M}_{0} A_{0}(q-\hat{q})-Q(G(p+q)-G(p))+q_{t}=0 .
$$

Então segue-se que

$$
\begin{aligned}
|-\Delta(q-\hat{q})| \leq & \kappa_{0}^{-1}|Q G(p+q)-Q G(p)|+\kappa_{0}^{-1}\left|q_{t}\right| \\
\leq & \kappa_{0}^{-1}\left|q_{t}\right|+\kappa_{0}^{-1}|k \wedge Q \vec{V}|+\kappa_{0}^{-1}|| Q z||+g_{0} \kappa_{0}^{-1}|\nabla \cdot Q \vec{V}|+ \\
& +\kappa_{0}^{-1}(|(P \vec{V} \cdot \nabla) Q \vec{V}|+|(Q \vec{V} \cdot \nabla) P \vec{V}|+|(Q \vec{V} \cdot \nabla) Q \vec{V}|)+ \\
& +\kappa_{0}^{-1}(|\nabla \cdot(P z Q \vec{V})|+|\nabla \cdot(Q z P \vec{V})|+|\nabla \cdot(Q z Q \vec{V})|),
\end{aligned}
$$

onde, na desigualdade anterior temos $p=\left(\begin{array}{c}P \vec{V} \\ P z\end{array}\right), \quad q=\left(\begin{array}{c}Q \vec{V} \\ Q z\end{array}\right)$.

Os termos são estimados usando E2, E3, E6 como segue,

$$
\begin{aligned}
|k \wedge Q \vec{V}| & \leq|Q \vec{V}| \leq \lambda_{m}^{-1 / 2}\|Q \vec{V}\| \leq \lambda_{m}^{-1 / 2}\|q\| \\
g_{0}|\nabla \cdot Q \vec{V}| & \leq \frac{3}{2} g_{0}\|Q \vec{V}\| \leq \frac{3}{2} g_{0}\|q\| \\
|(P \vec{V} \cdot \nabla) Q \vec{V}| & \leq c_{2}|P \vec{V}|^{1 / 2}|-\Delta P \vec{V}|^{1 / 2}\|Q \vec{V}\| \leq c_{2} \lambda_{1}^{-1 / 4}\|P \vec{V}\|^{1 / 2}|-\Delta P \vec{V}|^{1 / 2}\|Q \vec{V}\| \\
& \leq c_{2} \lambda_{1}^{-1 / 4} M_{1}^{1 / 2} M_{2}^{1 / 2}\|q\| \\
|(Q \vec{V} \cdot \nabla) P \vec{V}| & \leq c_{3}|Q \vec{V}|^{1 / 2}\|Q \vec{V}\|^{1 / 2}\|P \vec{V}\|^{1 / 2}|-\Delta P \vec{V}|^{1 / 2} \\
& \leq c_{3} \lambda_{m}^{-1 / 4}\|Q \vec{V}\|\|P \vec{V}\|^{1 / 2} \lambda_{m}^{1 / 4}\|P \vec{V}\|^{1 / 2} \\
& \leq c_{3}\|P \vec{V}\|\|Q \vec{V}\| \leq c_{3} M_{1}\|q\| \\
|(Q \vec{V} \cdot \nabla) Q \vec{V}| & \leq c_{2}|Q \vec{V}|^{1 / 2}|-\Delta Q \vec{V}|^{1 / 2}\|Q \vec{V}\| \leq c_{2} \lambda_{m}^{-1 / 4}\|Q \vec{V}\|^{1 / 2}|-\Delta Q \vec{V}|^{1 / 2}\|Q \vec{V}\| \\
& \leq c_{2} \lambda_{m}^{-1 / 4} M_{1}^{1 / 2} M_{2}^{1 / 2}\|q\|
\end{aligned}
$$


Os outros termos não lineares têm o mesmo tipo de tratamento:

$$
\begin{aligned}
& |\nabla \cdot(P z Q \vec{V})| \leq|P z \nabla \cdot Q \vec{V}|+|Q \vec{V} \cdot \nabla P z| \leq\left(c_{2} \lambda_{1}^{-1 / 4} M_{1}^{1 / 2} M_{2}^{1 / 2}+c_{3} M_{1}\right)\|q\| \\
& |\nabla \cdot(Q z P \vec{V})| \leq|Q z \nabla \cdot P \vec{V}|+|P \vec{V} \cdot \nabla Q z| \leq\left(c_{2} \lambda_{1}^{-1 / 4} M_{1}^{1 / 2} M_{2}^{1 / 2}+c_{3} M_{1}\right)\|q\| \\
& |\nabla \cdot(Q z Q \vec{V})| \leq|Q z \nabla \cdot Q \vec{V}|+|Q \vec{V} \cdot \nabla Q z| \leq 2 c_{2} \lambda_{m}^{-1 / 4} M_{1}^{1 / 2} M_{2}^{1 / 2}\|q\|
\end{aligned}
$$

Das estimativas previas obtemos $|-\Delta(q-\hat{q})| \leq \kappa_{0}^{-1} M_{6}\|q\|+\kappa_{0}^{-1}\left|q_{t}\right|$, onde

$$
M_{6}=3 c_{2}\left(\lambda_{1}^{-1 / 4}+\lambda_{m}^{-1 / 4}\right) M_{1}^{1 / 2} M_{2}^{1 / 2}+3 c_{3} M_{1}+\lambda_{m}^{-1 / 2}+\frac{3}{2} g_{0}
$$

o que conclui a prova do Lema (1.4).

Agora podemos estabelecer um dos resultados básicos da teoria de VIA,

Proposição 1.1 Satisfeitas as hipóteses dos Lemas (1.1)-(1.4), verifica-se a estimativa :

$$
|\xi-(p+\hat{q})|=|q-\hat{q}| \leq \lambda_{m}^{-3 / 2} M_{7}, \quad t \in[0, \infty)
$$

onde $M_{\tau}=\kappa_{0}^{-1}\left(M_{6} M_{2}+M_{5}\right)$ é uma constante calculada explicitamente em função de $f, \nu_{0}$ e dos dados iniciais $\xi_{0}$.

\section{Prova.}

Do Lema (1.4) temos,

$$
|\xi-(p+\hat{q})|=|q-\hat{q}| \leq \lambda_{m}^{-1}|-\Delta(q-\hat{q})| \leq \lambda_{m}^{-1} \kappa_{0}^{-1}\left(M_{6}\|q\|+\left|q_{t}\right|\right),
$$

e dos Lemas (1.1) e (1.2)

$$
\begin{aligned}
&\|q\| \leq \lambda_{m}^{-1 / 2}|-\Delta q| \leq \lambda_{m}^{-1 / 2} M_{2} \\
& \text { e } \quad\left|q_{t}\right| \leq \lambda_{m}^{-1 / 2}\left\|q_{t}\right\| \leq \lambda_{m}^{-1 / 2} M_{5}, \quad t \in[0, \infty) .
\end{aligned}
$$

concluímos que

$$
|\xi-(p+\hat{q})|=|q-\hat{q}| \leq \lambda_{m}^{-3 / 2} \kappa_{0}^{-1}\left(M_{6} M_{2}+M_{5}\right) \leq \lambda_{m}^{-3 / 2} M_{7}, \quad t \in[0, \infty) .
$$


O seguinte Lema limita a distância na norma do gradiente entre dois valores quaisquer do gráfico de $\Phi$,

Lema 1.5 Sejam $\left(p_{i}, q_{i}\right), i=1,2$, pertencentes ao gráfico de $\Phi$. Então

$$
\left\|q_{2}-q_{1}\right\| \leq \kappa_{0}^{-1}\left(M_{8}\left\|p_{1}\right\|+3 c_{1}\left|p_{2}-p_{1}\right|\right)\left\|p_{2}-p_{1}\right\|
$$

com $M_{8}$ constante dependendo de $\kappa_{0}$.

\section{$\underline{\text { Prova }}$}

Da definição de $\Phi$ temos: $\left\{\begin{array}{l}\mathbb{M}_{0} A_{0} q_{2}=Q G\left(p_{2}\right)+Q f \\ \mathbb{M}_{0} A_{0} q_{1}=Q G\left(p_{1}\right)+Q f\end{array}\right.$.

Em forma explícita, obtemos o sistema associado a $\left(p_{2}, q_{2}\right)$ :

$$
\begin{aligned}
& \nu_{0}(-\Delta) Q \vec{V}_{2}=Q\left(-\left(P \vec{V}_{2} \cdot \nabla\right) P \vec{V}_{2}-\vec{k} \wedge P \vec{V}_{2}-\nabla P z_{2}\right) \\
& \kappa_{0}(-\Delta) Q z_{2}=Q\left(-g_{0} \nabla \cdot\left(P \vec{V}_{2}\right)-\nabla \cdot\left(P z_{2} P \vec{V}_{2}\right)\right)+Q F .
\end{aligned}
$$

Observando que os termos $Q\left(-\vec{k} \wedge P \vec{V}_{2}\right), Q\left(\nabla P z_{2}\right)$ e $Q\left(\nabla \cdot P \vec{V}_{2}\right)$ se anulam, o sistema anterior é simplificado para:

$$
\begin{aligned}
& \nu_{0}(-\Delta) Q \vec{V}_{2}=Q\left(-\left(P \vec{V}_{2} \cdot \nabla\right) P \vec{V}_{2}\right) \\
& \kappa_{0}(-\Delta) Q z_{2}=Q\left(-\nabla \cdot\left(P z_{2} P \vec{V}_{2}\right)\right)+Q F
\end{aligned}
$$

Analogamente, obtemos:

$$
\begin{aligned}
& \nu_{0}(-\Delta) Q \vec{V}_{1}=Q\left(-\left(P \vec{V}_{1} \cdot \nabla\right) P \vec{V}_{1}\right) \\
& \kappa_{0}(-\Delta) Q z_{1}=Q\left(-\nabla \cdot\left(P z_{1} P \vec{V}_{1}\right)\right)+Q F .
\end{aligned}
$$

A diferença entre os dois leva a:

$$
\begin{aligned}
& \nu_{0}(-\Delta)\left(Q \vec{V}_{2}-Q \vec{V}_{1}\right)=-Q\left(-\left(P \vec{V}_{2} \cdot \nabla\right) P \vec{V}_{2}\right)+Q\left(-P \vec{V}_{1} \cdot \nabla P \vec{V}_{1}\right) \\
& \kappa_{0}(-\Delta)\left(Q z_{2}-Q z_{1}\right)=Q\left(-\nabla \cdot\left(P z_{2} P \vec{V}_{2}\right)\right)+Q\left(-\nabla \cdot\left(P z_{1} P \vec{V}_{1}\right)\right) .
\end{aligned}
$$

Tomando o produto escalar entre $\left(\begin{array}{c}Q \vec{V}_{2}-Q \vec{V}_{1} \\ Q z_{2}-Q z_{1}\end{array}\right)$ e o sistema anterior obtemos: 


$$
\begin{aligned}
\kappa_{0}\left\|q_{2}-q_{1}\right\|^{2} \leq & \left|\left(-\left(P \vec{V}_{2} \cdot \nabla\right) P \vec{V}_{2}+\left(P \vec{V}_{1} \cdot \nabla\right) P \vec{V}_{1}, Q \vec{V}_{2}-Q \vec{V}_{1}\right)\right|+ \\
& +\left|\left(\nabla \cdot\left(P z_{2} P \vec{V}_{2}-P z_{1} P \vec{V}_{1}\right), Q z_{2}-Q z_{1}\right)\right| \\
\leq \quad & \left|\left(\left(P \vec{V}_{1} \cdot \nabla\right)\left(P \vec{V}_{2}-P \vec{V}_{1}\right), Q \vec{V}_{2}-Q \vec{V}_{1}\right)\right|+ \\
& \left.+\mid\left(\left(P \overrightarrow{V_{2}}-P \vec{V}_{1}\right) \cdot \nabla\right) P \vec{V}_{1}, Q \vec{V}_{2}-Q \vec{V}_{1}\right) \mid+ \\
& +\left|\left(\left(\left(P \vec{V}_{2}-P \vec{V}_{1}\right) \cdot \nabla\right)\left(P \vec{V}_{2}-P \vec{V}_{1}\right), Q \vec{V}_{2}-Q \vec{V}_{1}\right)\right|+ \\
& +\left|\left(P z_{1} \nabla \cdot\left(P \vec{V}_{2}-P \vec{V}_{1}\right), Q z_{2}-Q z_{1}\right)\right|+ \\
& +\left|\left(\left(P z_{2}-P z_{1}\right) \nabla \cdot\left(P \vec{V}_{2}-P \vec{V}_{1}\right), Q z_{2}-Q z_{1}\right)\right|+ \\
& +\left|\left(P \vec{V}_{1} \cdot \nabla\left(P z_{2}-P z_{1}\right), Q z_{2}-Q z_{1}\right)\right|+ \\
& +\left|\left(\left(P \vec{V}_{2}-P \vec{V}_{1}\right) \cdot \nabla\left(P z_{2}-P z_{1}\right), Q z_{2}-Q z_{1}\right)\right|+ \\
& +\left|\left(\left(P \overrightarrow{V_{2}}-P \vec{V}_{1}\right) \cdot \nabla P z_{1}, Q z_{2}-Q z_{1}\right)\right|+ \\
& +\left|\left(\left(P z_{2}-P z_{1}\right) \nabla \cdot P \vec{V}_{1}, Q z_{2}-Q z_{1}\right)\right| \\
\leq\left(2 c_{1}(\right. & \left.\left.\lambda_{1} \lambda_{m}\right)^{-1 / 4}\left\|P \vec{V}_{1}\right\|+c_{1}\left|P \vec{V}_{2}-P \vec{V}_{1}\right|\right)\left\|P \vec{V}_{2}-P \vec{V}_{1}\right\|\left\|Q \vec{V}_{2}-Q \vec{V}_{1}\right\|+ \\
+\left(2 c_{1}(\right. & \left.\left.\lambda_{1} \lambda_{m}\right)^{-1 / 4}\left\|P z_{1}\right\|+c_{1}\left|P z_{2}-P z_{1}\right|\right)\left\|P \vec{V}_{2}-P \vec{V}_{1}\right\|\left\|Q z_{2}-Q z_{1}\right\|+ \\
+\left(2 c_{1}(\right. & \left.\left.\lambda_{1} \lambda_{m}\right)^{-1 / 4}\left\|P \vec{V}_{1}\right\|+c_{1}\left|P z_{2}-P z_{1}\right|\right)\left\|P z_{2}-P z_{1}\right\|\left\|Q z_{2}-Q z_{1}\right\|
\end{aligned}
$$

onde, na ultima desigualdade os termos foram estimados usando $E_{1}$. A simplificação da ultima expressão resulta em:

$$
\begin{aligned}
\left\|q_{2}-q_{1}\right\| & \leq \kappa_{0}^{-1}\left(6 c_{1}\left(\lambda_{1} \lambda_{m}\right)^{-1 / 4}\left\|p_{1}\right\|+3 c_{1}\left|p_{2}-p_{1}\right|\right)\left\|p_{2}-p_{1}\right\| \\
& \leq \kappa_{0}^{-1}\left(M_{8}\left\|p_{1}\right\|+3 c_{1}\left|p_{2}-p_{1}\right|\right)\left\|p_{2}-p_{1}\right\|
\end{aligned}
$$

onde $\quad M_{8}=6 c_{1}\left(\lambda_{1} \lambda_{m}\right)^{-1 / 4}$, completando a demonstração.

Observação 1.1 Se no Lema (1.5) tomarmos $q_{1}=\hat{q}$ e $q_{2}=\bar{q}$ (definidos em (1.62) $e$ (1.63) respectivamente), tem-se

$$
\|\hat{q}-\bar{q}\| \leq \kappa_{0}^{-1}\left(M_{8} M_{1}+3 c_{1}\left|p_{2}-p_{1}\right|\right)\left\|p_{2}-p_{1}\right\|
$$

onde usamos que $\left\|p_{1}\right\|=\|p\| \leq\|\xi\| \leq M_{1}$. 
1.5.1 Estimativas de erro para a aproximação por VIA das equações de água rasa

Serão analisadas as estimativas de erro para os modos baixos $|p-\bar{p}|$ e para os modos altos $|q-\bar{q}|$.

\subsubsection{Estimativa de erro para os modos baixos}

A estimativa de erro para $|p-\bar{p}|$ é sintetizada na seguinte proposição:

Proposição 1.2 Sob as hipóteses da Proposição (1.1), para um $T>0$, existem $m_{*}, M_{9}$ dependendo de $T, f, \nu_{0}, g_{0}, F$ e os dados iniciais, tais que para todo $m \geq m_{*}$, temos

$$
|\bar{p}-p| \leq M_{9} \lambda_{m}^{-3 / 2}, \quad t \in[0, T] .
$$

\section{$\underline{\text { Prova }}$}

Primeiro observe que dos Lemas (1.4), Lema (1.3) e Lema (1.2) resulta que:

$$
\begin{aligned}
\left|A_{0}(q-\hat{q})\right| & \leq \kappa_{0}^{-1} M_{6}\left|q_{t}\right|+\kappa_{0}^{-1}\|q\| \\
& \leq \kappa_{0}^{-1} M_{6} \lambda_{m}^{-1 / 2}\left\|q_{t}\right\|+\kappa_{0}^{-1} \lambda_{m}^{-1 / 2}\left|A_{0} q\right| \\
& \leq\left(\kappa_{0}^{-1} M_{6} M_{5}+\kappa_{0}^{-1} M_{2}\right) \lambda_{m}^{-1 / 2}=M_{6}^{\prime} \lambda_{m}^{-1 / 2}
\end{aligned}
$$

Sendo $w=\left(\begin{array}{c}w_{1} \\ w_{2}\end{array}\right)=\left(\begin{array}{c}P \vec{V}-P \vec{V} \\ P z-P \bar{z}\end{array}\right)$, da observação (1.1) vemos que

$$
\|\bar{q}-\hat{q}\| \leq \kappa_{0}^{-1}\left(M_{8} M_{1}+3 c_{1}|w|\right)\|w\| .
$$

Subtraindo ( 1.60$)$ de (1.57) temos

$$
\frac{d w}{d t}+M_{0} A_{0} w=P G(p+q)-P G(\bar{p}+\bar{q}) .
$$

Tomando o produto interno com respeito a $w$ resulta,

$$
\left.\frac{1}{2} \frac{d}{d t}|w|^{2}+\kappa_{0}\|w\|^{2} \leq \mid(P G(p+q)-P G(\bar{p}+\bar{q})), w\right) \mid .
$$


O termo $S=P G(p+q)-P G(\bar{p}+\bar{q})$ pode ser escrito como,

$$
S=P\left(\begin{array}{l}
-(\vec{V} \cdot \nabla) \vec{V}+(\overrightarrow{\vec{V}} \cdot \nabla) \overrightarrow{\vec{V}}-\nabla(z-\bar{z}) \\
-g_{0} \nabla \cdot(\vec{V}-\overrightarrow{\vec{V}})-\nabla \cdot(z \vec{V})+\nabla \cdot(\vec{z} \vec{V})
\end{array}\right)
$$

Os termos $\nabla(Q z-Q \bar{z}), \quad K \wedge(Q \vec{V}-Q \overrightarrow{\vec{V}}), \quad g_{0} \nabla \cdot(Q \vec{V}-Q \overrightarrow{\vec{V}})$ são ortogonais a $w$, e por isso não são considerados na expressão anterior.

Os termos não lineares são agrupados e decompostos como segue,

$$
\begin{aligned}
-(\vec{V} \cdot \nabla) \vec{V}+(\vec{V} \cdot \nabla) \vec{V}= & -(\vec{V} \cdot \nabla)(\vec{V}-\vec{V})-((\vec{V}-\vec{V}) \cdot \nabla) \vec{V}+((\vec{V}-\vec{V}) \cdot \nabla)(\vec{V}-\vec{V}) \\
= & -(\vec{V} \cdot \nabla) w_{1}-(\vec{V} \cdot \nabla)(Q \vec{V}-Q \overrightarrow{\vec{V}})-\left(w_{1} \cdot \nabla\right) \vec{V}+w_{1} \cdot \nabla w_{1}- \\
& -((Q \vec{V}-Q \overrightarrow{\vec{V}}) \cdot \nabla) \vec{V}+\left(w_{1} \cdot \nabla\right)(Q \vec{V}-Q \overrightarrow{\vec{V}})+ \\
& +((Q \vec{V}-Q \overrightarrow{\vec{V}}) \cdot \nabla) w_{1}+((Q \vec{V}-Q \overrightarrow{\vec{V}}) \cdot \nabla)(Q \vec{V}-Q \overrightarrow{\vec{V}}) \\
-\nabla \cdot(z \vec{V})+\nabla \cdot(\bar{z} \vec{V})= & (z-\bar{z}) \nabla \cdot(\vec{V}-\overrightarrow{\vec{V}})+(\vec{V}-\vec{V}) \cdot \nabla(z-\bar{z})-z \nabla \cdot(\vec{V}-\overrightarrow{\vec{V}})- \\
& -\vec{V} \cdot \nabla(z-\bar{z})-(\vec{V}-\overrightarrow{\vec{V}}) \cdot \nabla z-(z-\bar{z}) \nabla \cdot \vec{V} \\
= & w_{2} \nabla \cdot w_{1}+w_{2} \nabla \cdot(Q \vec{V}-Q \overrightarrow{\vec{V}})+(Q z-Q \bar{z}) \nabla \cdot w_{1}- \\
& -z \nabla \cdot w_{1}+(Q z-Q \bar{z}) \nabla \cdot(Q \vec{V}-Q \overrightarrow{\vec{V}})+w_{1} \cdot \nabla w_{2}+ \\
& +w_{1} \cdot \nabla(Q z-Q \bar{z})+(Q \vec{V}-Q \overrightarrow{\vec{V}}) \cdot \nabla w_{2}-z \nabla \cdot(Q \vec{V}-Q \overrightarrow{\vec{V}})+ \\
& +(Q \vec{V}-Q \overrightarrow{\vec{V}}) \cdot \nabla(Q z-Q \bar{z})-\vec{V} \cdot \nabla w_{2}-\vec{V} \cdot \nabla(Q z-Q \bar{z})- \\
& -w_{1} \cdot \nabla z-(Q \vec{V}-Q \overrightarrow{\vec{V}}) \cdot \nabla z-w_{2} \nabla \cdot \vec{V}-(Q z-Q \bar{z}) \nabla \cdot \vec{V}
\end{aligned}
$$

Faltam ainda, para obter o erro da ordem $\lambda_{m}^{-3 / 2}$, as estimativas seguintes:

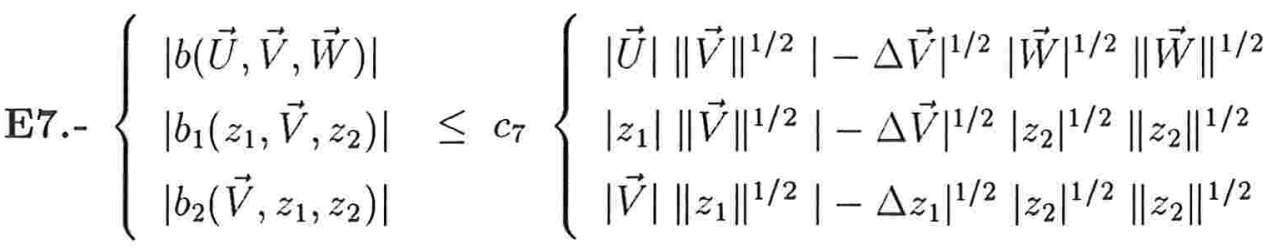

\section{Prova.-}

O resultado segue de $\mathbf{E} 2$, observando-se que

$|b(\vec{U}, \vec{V}, \vec{W})| \leq \sum_{i, j=1}^{2}\left|u_{i}\right|_{L_{2}}\left|D_{i} v_{j}\right|_{L_{4}}\left|w_{j}\right|_{L_{4}} \leq 4|\vec{U}|\|\vec{V}\|_{L_{4}}|\vec{W}|_{L_{4}}$

A prova para $b_{1}$ e $b_{2}$ é similar à prova de $b$. 
E8.- $\left\{\begin{array}{l}b(\vec{U}, \vec{V}, \vec{W})=-b(\vec{U}, \vec{W}, \vec{V})-\int_{D}(\nabla \cdot \vec{U})(\vec{V} \cdot \vec{W}) d \Omega \\ b_{1}\left(z_{1}, \vec{V}, z_{2}\right)+b_{2}\left(\vec{V}, z_{1}, z_{2}\right)+b_{2}\left(\vec{V}, z_{2}, z_{1}\right)=0 \\ \left|\int_{D}(\nabla \cdot \vec{U})(\vec{V} \cdot \vec{W}) d \Omega\right| \leq c_{8}\|\vec{U}\|^{1 / 2}|-\Delta \vec{U}|^{1 / 2}|\vec{V}||\vec{W}|^{1 / 2}\|\vec{W}\|^{1 / 2}\end{array}\right.$

Prova.-

As duas primeiras decorrem da seguinte identidade:

$$
D_{i}\left(u_{i} v_{j} w_{j}\right)=\left(D_{i} u_{i}\right) v_{j} w_{j}+u_{i}\left(D_{i} v_{j}\right) w_{j}+u_{i} v_{j}\left(D_{i} w_{j}\right)
$$

e a ultima decorre de estimar $\vec{U}, \vec{V}$ e $\vec{W}$ nas normas $L_{4}, L_{2}$ e $L_{4}$ respectivamente.

Os termos são estimados usando as estimativas de Sobolev E1-E8, os Lemas anteriores, as estimativas (1.64), (1.65), o Lema de Gronwall, e decompondo $q-\bar{q}=(q-\hat{q})+(\hat{q}-\bar{q})$ :

b1.- $\left|\left((\vec{V} \cdot \nabla) w_{i}, w_{i}\right)\right| \leq c_{2}|\vec{V}|^{1 / 2}|-\Delta \vec{V}|^{1 / 2}\left|w_{i}\right|\left\|w_{i}\right\| \leq c_{2} M_{1}^{1 / 2} M_{2}^{1 / 2}|w|\|w\|$

$$
\leq \frac{\epsilon}{2}\|w\|^{2}+\frac{1}{2 \epsilon} c_{1}^{2} M_{1} M_{2}|w|^{2}
$$

b2.- $\left|\left((\vec{V} \cdot \nabla)(Q \vec{V}-Q \overrightarrow{\hat{V}}), w_{1}\right)\right|=\left|b\left(\vec{V}, w_{1}, Q \vec{V}-Q \overrightarrow{\hat{V}}\right)+\right| \int_{D}(\nabla \cdot \vec{V}) w_{1} \cdot(Q \vec{V}-Q \overrightarrow{\hat{V}}) d \Omega \mid$ $\leq c_{2}|\vec{V}|^{1 / 2}|-\Delta \vec{V}|^{1 / 2}\|w\||q-\hat{q}|+c_{8}\|\vec{V}\|^{1 / 2}|-\Delta \vec{V}|^{1 / 2}|q-\hat{q}||w|^{1 / 2}\|w\|^{1 / 2}$ $\leq\left(c_{2}+c_{8}\right) \lambda_{1}^{-1 / 4} M_{1}^{1 / 2} M_{2}^{1 / 2} M_{7} \lambda_{m}^{-3 / 2}\|w\|$ $\leq \frac{\epsilon}{2}\|w\|^{2}+\frac{1}{2 \epsilon}\left(c_{2}+c_{8}\right)^{2} \lambda_{1}^{-1 / 2} M_{1} M_{2} M_{7}^{2} \lambda_{m}^{-3}$

b3.- $\left|\left((\vec{V} \cdot \nabla)(Q \vec{V}-Q \overrightarrow{\hat{V}}), w_{1}\right)\right|=\left|b\left(\vec{V}, w_{1}, Q \vec{V}-Q \overrightarrow{\hat{V}}\right)+\right| \int_{D}(\nabla \cdot \vec{V})(Q \vec{V}-Q \overrightarrow{\hat{V}}) \cdot w_{1} d \Omega \mid$ $\leq c_{2}|\vec{V}|^{1 / 2}|-\Delta \vec{V}|^{1 / 2}|| w\left\||\vec{q}-\hat{q}|+c_{8}\right\| \vec{V}\left\|^{1 / 2}|-\Delta \vec{V}|^{1 / 2}|\bar{q}-\hat{q}||w|^{1 / 2}\right\| w \|^{1 / 2}$ $\leq\left(c_{2}+c_{8}\right) \lambda_{1}^{-1 / 4} M_{1}^{1 / 2} M_{2}^{1 / 2} \lambda_{m}^{-1 / 2}\|\bar{q}-\hat{q}\|\|w\|$ $\leq\left(c_{2}+c_{8}\right) \lambda_{1}^{-1 / 4} M_{1}^{1 / 2} M_{2}^{1 / 2} \kappa_{0}^{-1} \lambda_{m}^{-1 / 2}\left(M_{8} M_{1}+3 c_{1}|w|\right)\|w\|^{2}$

b4.- $\left|\left(\left(w_{i} \cdot \nabla\right) \vec{V}, w_{j}\right)\right| \leq c_{1}\left|w_{i}\right|^{1 / 2}\left\|w_{i}\right\|^{1 / 2}\|\vec{V}\|\left|w_{j}\right|^{1 / 2}\left\|w_{j}\right\|^{1 / 2} \leq c_{1} M_{1}|w|\|w\|$

$$
\leq \frac{\epsilon}{2}\|w\|^{2}+\frac{1}{2 \epsilon} c_{1}^{2} M_{1}^{2}\left|w_{1}\right|^{2}
$$

b5.- $\left|\left(((Q \vec{V}-Q \overrightarrow{\hat{V}}) \cdot \nabla) \vec{V}, w_{1}\right)\right| \leq c_{7}|q-\hat{q}|\|\vec{V}\|^{1 / 2}|-\Delta \vec{V}|^{1 / 2}|w|^{1 / 2}\|w\|^{1 / 2}$

$$
\begin{aligned}
& \leq c_{7} M_{1}^{1 / 2} M_{2}^{1 / 2} M_{7} \lambda_{1}^{-1 / 4} \lambda_{m}^{-3 / 2}\|w\| \\
& \leq \frac{\epsilon}{2}\|w\|^{2}+\frac{1}{2 \epsilon} c_{7}^{2} M_{1} M_{2} M_{7}^{2} \lambda_{1}^{-1 / 2} \lambda_{m}^{-3}
\end{aligned}
$$

b6. $\left|\left(((Q \overrightarrow{\vec{V}}-Q \overrightarrow{\hat{V}}) \cdot \nabla) \vec{V}, w_{1}\right)\right| \leq c_{7} \lambda_{m}^{-1 / 2}\|\vec{q}-\hat{q}\|\|\vec{V}\|^{1 / 2}|-\Delta \vec{V}|^{1 / 2}|w|^{1 / 2}\|w\|^{1 / 2}$

$$
\leq c_{7} M_{1}^{1 / 2} M_{2}^{1 / 2} \lambda_{1}^{-1 / 4} \lambda_{m}^{-1 / 2} \kappa_{0}^{-1}\left(M_{8} M_{1}+3 c_{1}|w|\right)\|w\|^{2}
$$


b7.- $\left|\left(w_{1} \cdot \nabla w_{i}, w_{i}\right)\right| \leq c_{1}\left|w_{1}\right|^{1 / 2}\left\|w_{1}\right\|^{1 / 2}\left\|w_{i}\right\|\left|w_{i}\right|^{1 / 2}\left\|w_{i}\right\|^{1 / 2} \leq c_{1}|w|\|w\|^{2}$

b8.- $\left|\left(\left(w_{1} \cdot \nabla\right)(Q \vec{V}-Q \overrightarrow{\hat{V}}), w_{1}\right)\right| \leq c_{1}\|q-\hat{q}\||w|\|w\| \leq c_{1} \lambda_{m}^{-1 / 2}|-\Delta(q-\hat{q})||w|\|w\|$ $\leq c_{1} M_{6}^{\prime} \lambda_{1}^{-1 / 2} \lambda_{m}^{-1}\|w\|^{2}$

b9.- $\left|\left(\left(w_{1} \cdot \nabla\right)(Q \overrightarrow{\vec{V}}-Q \overrightarrow{\hat{V}}), w_{1}\right)\right| \leq c_{1}\|\bar{q}-\hat{q}\||w|\|w\|$

$$
\leq c_{1} \kappa_{0}^{-1}\left(M_{8} M_{1}|w|+3 c_{1}|w|^{2}\right)\|w\|^{2}
$$

b10.- $\left|\left(((Q \vec{V}-Q \overrightarrow{\hat{V}}) \cdot \nabla) w_{i}, w_{i}\right)\right| \leq c_{2}|q-\hat{q}|^{1 / 2}|-\Delta(q-\hat{q})|^{1 / 2}|| w|||w|$

$$
\leq c_{2} \lambda_{1}^{-1 / 2} M_{7}^{1 / 2}\left(M_{6}^{\prime}\right)^{1 / 2} \lambda_{m}^{-1}\|w\|^{2}
$$

b11.- $\left|\left(((Q \overrightarrow{\vec{V}}-Q \overrightarrow{\hat{V}}) \cdot \nabla) w_{i}, w_{i}\right)\right| \leq c_{1} \lambda_{m}^{-1 / 4}\|\bar{q}-\hat{q}\|\|w\||w|^{1 / 2}\|w\|^{1 / 2}$

$$
\begin{aligned}
& \leq c_{1} \lambda_{m}^{-1 / 4} \kappa_{0}^{-1}\left(M_{8} M_{1}+3 c_{1}|w|\right) \lambda_{m}^{1 / 4}|w|\|w\|^{2} \\
& \leq c_{1} \kappa_{0}^{-1}\left(M_{8} M_{1}|w|+3 c_{1}|w|^{2}\right)\|w\|^{2}
\end{aligned}
$$

b12.- $\left.\mid((Q \vec{V}-Q \overrightarrow{\hat{V}}) \cdot \nabla)(Q \vec{V}-Q \overrightarrow{\hat{V}}), w_{1}\right)\left|\leq c_{1}\right| q-\left.\hat{q}\right|^{1 / 2}\|q-\hat{q}\|^{3 / 2}|w|^{1 / 2}\|w\|^{1 / 2}$

$$
\begin{aligned}
& \leq c_{1} \lambda_{1}^{-1 / 4} M_{7}^{1 / 2}\left(M_{6}^{\prime}\right)^{3 / 2} \lambda_{m}^{-9 / 4}\|w\| \\
& \leq \frac{\epsilon}{2}\|w\|^{2}+\frac{1}{2 \epsilon} c_{1}^{2} \lambda_{1}^{-1 / 2} M_{7}\left(M_{6}^{\prime}\right)^{3} \lambda_{m}^{-9 / 2}
\end{aligned}
$$

b13.- $\left|\left(((Q \vec{V}-Q \overrightarrow{\hat{V}}) \cdot \nabla)(Q \vec{V}-Q \overrightarrow{\hat{V}}), w_{1}\right)\right| \leq c_{1} \lambda_{1}^{-1 / 4} \lambda_{m}^{-3 / 4}|-\Delta(q-\hat{q})|\|\bar{q}-\hat{q}\|\|w\|$

$$
\leq c_{1} M_{6}^{\prime} \kappa_{0}^{-1} \lambda_{1}^{-1 / 4} \lambda_{m}^{-5 / 4}\left(M_{8} M_{1}+3 c_{1}|w|\right)\|w\|^{2}
$$

b14.- $\left|\left(((Q \overrightarrow{\vec{V}}-Q \overrightarrow{\hat{V}}) \cdot \nabla)(Q \overrightarrow{\vec{V}}-Q \overrightarrow{\hat{V}}), w_{1}\right)\right| \leq c_{1} \lambda_{m}^{-1 / 4}\|\bar{q}-\hat{q}\|^{2} \lambda_{m}^{1 / 4}|w|$

$$
\leq 2 c_{1} \kappa_{0}^{-2}\left(M_{8}^{2} M_{1}^{2}|w|+9 c_{1}^{2}|w|^{3}\right)\|w\|^{2}
$$

onde $i, j=1$, ou 2 . Os outros termos verificam algumas das estimativas anteriores (b2, b3, b5, b6, b12 e b15 tem cada uma outros dois termos com estimativas similares, b13 com cinco, e b8, b9 com um termo similar respectivamente).

Tomando $\epsilon=\kappa_{0} / 14$, obtém-se um termo da forma $\kappa_{0}\|w\|^{2} / 2$, o qual é absorvido no lado direito. A estimativa final é do tipo:

$$
\frac{d}{d t}|w|^{2}+\kappa_{0}\|w\|^{2} \leq \alpha|w|^{2}+\left(r_{1} \lambda_{m}^{-1 / 2}+r_{2}|w|+r_{3}|w|^{2}+r_{4}|w|^{3}\right)\|w\|^{2}+s \lambda_{m}^{-3}
$$


Usamos a seguir um argumento de linearidade. Para $T>0$ fixo temos:

$$
\begin{gathered}
\frac{d}{d t}|w|^{2} \leq \alpha|w|^{2}+s \lambda_{m}^{-3}, \\
|w|^{2} \leq \frac{s \lambda_{m}^{-3}}{\alpha}\left(e^{\alpha t}-1\right)=K(T) \lambda_{m}^{-3}
\end{gathered}
$$

Escolhendo $m^{*}$ tal que:

$$
|w| \leq 1 ; \quad\left(\text { ou } K(T) \lambda_{m}^{-3} \leq 1\right)
$$

e,

$$
r_{1} \lambda_{m}^{-1 / 2} \leq \frac{\kappa_{0}}{8}, \quad \max \left\{r_{2}, r_{3}, r_{4}\right\}|w| \leq \frac{\kappa_{0}}{8},
$$

os termos não lineares são absorvidos no lado direito, levando a:

$$
\frac{d}{d t}|w|^{2}+\frac{1}{2} \kappa_{0}\|w\|^{2} \leq \alpha|w|^{2}+s \lambda_{m}^{-3}
$$

Integrando, temos para $T>0$ :

$$
|w|^{2} \leq K(T) \lambda_{m}^{-3} \Rightarrow|w| \leq K^{1 / 2}(T) \lambda_{m}^{-3 / 2}
$$

Com $M_{9}=K^{-1 / 2}(T)$ é completada a prova da Proposição. 


\subsubsection{Estimativa de erro para os modos altos}

A estimativa de erro para os modos altos é uma conseqüência da Proposição (1.2):

Lema 1.6 Sob as hipóteses da Proposição (1.2) se verifica a estimativa:

$$
|q-\bar{q}| \leq M_{10} \lambda_{m}^{-3 / 2}
$$

Prova.-

$$
\begin{array}{rlr}
|q-\hat{q}| & \leq \lambda_{m}^{-1}\|q-\hat{q}\| \leq M_{6}^{\prime} \lambda_{m}^{-3 / 2}, & \text { por }(1.64) \\
|\bar{q}-\hat{q}| & \leq \lambda_{m}^{-1 / 2} \kappa_{0}^{-1}\left(M_{8} M_{1}+3 c_{1}|w|\right)\|w\| & \text { por }(1.65) \\
& \leq \lambda_{m}^{-1 / 2} \kappa_{0}^{-1}\left(M_{8} M_{1}+3 c_{1} M_{9} \lambda_{m}^{-3 / 2}\right) \lambda_{m}^{1 / 2} M_{9} \lambda_{m}^{-3 / 2} \\
& \leq \kappa_{0}^{-1}\left(M_{8} M_{1}+3 c_{1} \lambda_{m}^{-3 / 2} M_{9}\right) M_{9} \lambda_{m}^{-3 / 2}
\end{array}
$$

Logo,

$$
\begin{aligned}
|q-\bar{q}| & \leq|q-\hat{q}|+|\bar{q}-\hat{q}| \\
& \leq M_{10} \lambda_{m}^{-3 / 2} .
\end{aligned}
$$

onde $M_{10}=M_{6}^{\prime}+\kappa_{0}^{-1}\left(M_{8} M_{1}+3 c_{1} \lambda_{m}^{-3 / 2} M_{9}\right) M_{9}$, completando a demonstração.

Teorema 1.2 Da Proposição (1.2) e do Lema (1.6), temos a estimativa de erro entre a solução $\xi$ do sistema original, e a solução $\bar{\xi}$ do sistema aproximado por Galerkin não linear:

$$
|\xi-\bar{\xi}|=|(p+q)-(\bar{p}+\bar{q})| \leq|p-\bar{p}|+|q-\bar{q}| \leq\left(M_{9}+M_{10}\right) \lambda_{m}^{-3 / 2}
$$




\subsubsection{Estimativa de erro para a aproximação por Galerkin linear}

Analisamos aqui a estimativa de erro entre as soluções $p+q$ do sistema original (1.57)(1.58) e a aproximação $\tilde{p}$ pelo método de Galerkin linear (1.59). Inicialmenteé estabelecida a seguinte estimativa para a componente dos modos altos $q$ do sistema original:

Lema 1.7 Para $t>0$ são satisfeitas as seguintes estimativas:

$$
|q| \leq M_{2} \lambda_{m}^{-1}, \quad\|q\| \leq M_{2} \lambda_{m}^{-1 / 2}, \quad\left|q_{t}\right| \leq M_{5} \lambda_{m}^{-1 / 2}
$$

$\underline{\text { Prova }}$

Dos Lemas (1.1) e (1.3) tem-se $|-\Delta \xi| \leq M_{2}$ e $\left\|\xi_{t}\right\| \leq M_{5}$, o que junto com a estimativa E6 implica:

$$
\begin{aligned}
|q| & \leq|\xi| \leq \lambda_{m}^{-1}|-\Delta \xi| \leq M_{2} \lambda_{m}^{-1} \\
\|q\| & \leq\|\xi\| \leq \lambda_{m}^{-1 / 2}|-\Delta \xi| \leq M_{2} \lambda_{m}^{-1 / 2} \\
\left|q_{t}\right| & \leq\left|\xi_{t}\right| \leq \lambda_{m}^{-1 / 2}\left\|\xi_{t}\right\| \leq M_{5} \lambda_{m}^{-1 / 2}
\end{aligned}
$$

o que completa a prova do Lema.

Com base no Lema anterior estabelecemos a seguinte estimativa de erro:

Proposição 1.3 Para $T>0$, existem $m_{*}, M_{11}$ dependendo de $T, f, \nu_{0}, g_{0}, F$ e os dados iniciais, tais que para todo $m \geq m_{*}$, temos

$$
|\tilde{p}-p| \leq M_{11} \lambda_{m}^{-1}, \quad t \in[0, T]
$$

$\underline{\text { Prova }}$

$$
\begin{aligned}
\text { Sendo } w=\left(\begin{array}{c}
w_{1} \\
w_{2}
\end{array}\right)= & \left(\begin{array}{c}
P \vec{V}-P \overrightarrow{\tilde{V}} \\
P z-P \tilde{z}
\end{array}\right), \text { e subtraindo (1.59) de (1.57) temos } \\
& \frac{d w}{d t}+M_{0} A_{0} w=P G(p+q)-P G(\tilde{p}) .
\end{aligned}
$$

Tomando o produto interno com respeito a $w$ resulta,

$$
\left.\frac{1}{2} \frac{d}{d t}|w|^{2}+\kappa_{0}\|w\|^{2} \leq \mid(P G(p+q)-P G(\tilde{p})), w\right) \mid .
$$


O termo $S=P G(p+q)-P G(\tilde{p})$ pode ser escrito como,

$$
S=P\left(\begin{array}{l}
-(\vec{V} \cdot \nabla) \vec{V}+(\overrightarrow{\tilde{V}} \cdot \nabla) \overrightarrow{\tilde{V}}-\nabla(z-\tilde{z}) \\
-g_{0} \nabla \cdot(\vec{V}-\overrightarrow{\tilde{V}})-\nabla \cdot(z \vec{V})+\nabla \cdot(\tilde{z} \overrightarrow{\tilde{V}})
\end{array}\right)
$$

Os termos $\nabla(Q z), \quad K \wedge(Q \vec{V}), \quad g_{0} \nabla \cdot(Q \vec{V})$ são ortogonais a $w$, e por isso não são considerados na expressão anterior.

Os termos não lineares são agrupados e decompostos como segue,

$$
\begin{aligned}
-(\vec{V} \cdot \nabla) \vec{V}+(\overrightarrow{\tilde{V}} \cdot \nabla) \overrightarrow{\tilde{V}}= & -(\vec{V} \cdot \nabla)(\vec{V}-\overrightarrow{\tilde{V}})-((\vec{V}-\overrightarrow{\tilde{V}}) \cdot \nabla) \vec{V}+((\vec{V}-\overrightarrow{\tilde{V}}) \cdot \nabla)(\vec{V}-\overrightarrow{\tilde{V}}) \\
= & -(P \vec{V} \cdot \nabla) w_{1}-(P \vec{V} \cdot \nabla) Q \vec{V}-\left(w_{1} \cdot \nabla\right) P \vec{V}+w_{1} \cdot \nabla w_{1}- \\
& -(Q \vec{V} \cdot \nabla) \vec{V} \\
-\nabla \cdot(z \vec{V})+\nabla \cdot(\tilde{z} \vec{V})= & (z-\tilde{z}) \nabla \cdot(\vec{V}-\overrightarrow{\tilde{V}})+(\vec{V}-\overrightarrow{\tilde{V}}) \cdot \nabla(z-\tilde{z})-z \nabla \cdot(\vec{V}-\overrightarrow{\tilde{V}})- \\
& -\vec{V} \cdot \nabla(z-\tilde{z})-(\vec{V}-\overrightarrow{\tilde{V}}) \cdot \nabla z-(z-\tilde{z}) \nabla \cdot \vec{V} \\
= & w_{2} \nabla \cdot w_{1}-w_{2} \nabla \cdot P \vec{V}-P z \nabla \cdot w_{1}-Q z \nabla \cdot P \vec{V}+w_{1} \cdot \nabla w_{2}- \\
& -w_{1} \cdot \nabla P z-P \vec{V} \cdot \nabla w_{2}-z \nabla \cdot Q \vec{V}-Q \vec{V} \cdot \nabla P z-\vec{V} \cdot \nabla Q z
\end{aligned}
$$

Os termos são estimados usando as estimativas de Sobolev E1-E8, os Lemas anteriores e o Lema de Gronwall:

b1.- $\left|\left((P \vec{V} \cdot \nabla) w_{1}, w_{1}\right)\right| \leq c_{2}|\vec{V}|^{1 / 2}|-\Delta \vec{V}|^{1 / 2}\left|w_{1}\right|\left\|w_{1}\right\| \leq c_{2} M_{1}^{1 / 2} M_{2}^{1 / 2}|w|\|w\|$

$$
\leq \frac{\epsilon}{2}\|w\|^{2}+\frac{1}{2 \epsilon} c_{1}^{2} M_{1} M_{2}|w|^{2}
$$

b2.- $\left|\left((P \vec{V} \cdot \nabla) Q \vec{V}, w_{1}\right)\right|=\left|b\left(P \vec{V}, w_{1}, Q \vec{V}\right)+\right| \int_{D}(\nabla \cdot P \vec{V}) w_{1} \cdot Q \vec{V} d \Omega \mid$

$$
\begin{aligned}
& \leq c_{2}|\vec{V}|^{1 / 2}|-\Delta \vec{V}|^{1 / 2}\|w\||q|+c_{8}\|\vec{V}\|^{1 / 2}|-\Delta \vec{V}|^{1 / 2}|q||w|^{1 / 2}\|w\|^{1 / 2} \\
& \leq\left(c_{2}+c_{8}\right) \lambda_{1}^{-1 / 4} M_{1}^{1 / 2} M_{2}^{3 / 2} \lambda_{m}^{-1}\|w\| \\
& \leq \frac{\epsilon}{2}\|w\|^{2}+\frac{1}{2 \epsilon}\left(c_{2}+c_{8}\right)^{2} \lambda_{1}^{-1 / 2} M_{1} M_{2}^{3} \lambda_{m}^{-2}
\end{aligned}
$$

b3.- $\left|\left(\left(w_{1} \cdot \nabla\right) P \vec{V}, w_{1}\right)\right| \leq c_{1}\left|w_{1}\right|^{1 / 2}\left\|w_{1}\right\|^{1 / 2}\|\vec{V}\|\left|w_{1}\right|^{1 / 2}\left\|w_{1}\right\|^{1 / 2} \leq c_{1} M_{1}|w|\|w\|$

$$
\leq \frac{\epsilon}{2}\|w\|^{2}+\frac{1}{2 \epsilon} c_{1}^{2} M_{1}^{2}\left|w_{1}\right|^{2}
$$

b4.- $\left|\left(w_{1} \cdot \nabla w_{1}, w_{1}\right)\right| \leq c_{1}\left|w_{1}\right|^{1 / 2}\left\|w_{1}\right\|^{1 / 2}\left\|w_{1}\right\|\left|w_{1}\right|^{1 / 2}\left\|w_{1}\right\|^{1 / 2} \leq c_{1}|w|\|w\|^{2}$ 


$$
\begin{aligned}
\text { b5.- }\left|\left((Q \vec{V} \cdot \nabla) \vec{V}, w_{1}\right)\right| & \leq c_{7}|q|\|\vec{V}\|^{1 / 2}|-\Delta \vec{V}|^{1 / 2}|w|^{1 / 2}\|w\|^{1 / 2} \\
& \leq c_{7} M_{1}^{1 / 2} M_{2}^{3 / 2} \lambda_{1}^{-1 / 4} \lambda_{m}^{-1}\|w\| \\
& \leq \frac{\epsilon}{2}\|w\|^{2}+\frac{1}{2 \epsilon} c_{7}^{2} M_{1} M_{2}^{3} \lambda_{1}^{-1 / 2} \lambda_{m}^{-2} .
\end{aligned}
$$

Cada um dos outros termos corresponde a alguma das estimativas anteriores, tomando $\epsilon=\kappa_{0} / 12$, obtém-se um termo da forma $\kappa_{0}\|w\|^{2} / 2$, o que é absorvido no lado direito. A estimativa final é do tipo:

$$
\frac{d}{d t}|w|^{2}+\kappa_{0}\|w\|^{2} \leq \alpha|w|^{2}+\alpha_{1}|w|\|w\|^{2}+\alpha_{2} \lambda_{m}^{-2}
$$

Usamos a seguir o mesmo argumento de linearidade da prova da Proposição (1.2). Para $T>0$ fixo temos:

$$
\begin{gathered}
\frac{d}{d t}|w|^{2} \leq \alpha|w|^{2}+\alpha_{2} \lambda_{m}^{-2} \\
|w|^{2} \leq \frac{\alpha_{2} \lambda_{m}^{-2}}{\alpha}\left(e^{\alpha t}-1\right)=K(T) \lambda_{m}^{-2}
\end{gathered}
$$

Escolhendo $m^{*}$ tal que:

$$
|w| \leq 1 ; \quad\left(\text { ou } K(T) \lambda_{m}^{-2} \leq 1\right)
$$

e, $\quad \alpha_{1}|w| \leq \frac{\kappa_{0}}{2}$, os termos não lineares são absorvidos no lado direito, levando a:

$$
\frac{d}{d t}|w|^{2}+\frac{1}{2} \kappa_{0}\|w\|^{2} \leq \alpha|w|^{2}+\alpha_{2} \lambda_{m}^{-2}
$$

Integrando, temos para $T>0$ :

$$
|w|^{2} \leq K(T) \lambda_{m}^{-2} \Rightarrow|w| \leq K^{1 / 2}(T) \lambda_{m}^{-1}
$$

e com a escolha de $M_{11}=K^{1 / 2}(T)$ é completada demonstração.

Da Proposição (1.3) e o Lema (1.7) temos:

Teorema 1.3 O erro entre a solução $\xi$ do sistema original e a solução $\tilde{p}$ do sistema aproximado por Galerkin Linear satisfaz a seguinte estimativa:

$$
|\xi-\tilde{p}|=|(p+q)-\tilde{p}| \leq|p-\tilde{p}|+|q| \leq\left(M_{2}+M_{11}\right) \lambda_{m}^{-1} .
$$




\section{Capítulo 2}

\section{Método numérico de Galerkin Não}

\section{Linear}

No artigo de Foias, Manley e Teman [23] foram apresentados novos conceitos: variedades inerciais aproximadas no contexto dos sistemas dinâmicos e suas aplicações a fenômenos de turbulência, e no artigo de Marion e Temam [48] foram propostos uma nova família de algorítmos numéricos chamados métodos de Galerkin não linear. Estes conceitos e métodos são baseados na decomposição das variáveis (solução procurada de uma equação diferencial), tal como o campo de velocidade, em suas componentes de pequena escala (modos altos) e de grande escala (ou modos baixos). Essencialmente o método de Galerkin não linear é constituído por um tratamento diferenciado das pequenas e grandes escalas (ou equivalentemente dos modos altos e baixos). Na literatura existente geralmente aplica-se o método a equações de evolução do tipo:

$$
\frac{\partial u}{\partial t}+A u+B(u, u)=f
$$

com condição inicial $u(0)=u_{0}$. O operador $A$ é um operador positivo autoadjunto com inversa compacta. Nestas condições existe um conjunto ortonormal completo de autofunções $\left\{\mathrm{v}_{j}\right\}$ de $A$ tal que $A v_{j}=\lambda_{j} \mathrm{v}_{j}$ e $0<\lambda_{1} \leq \lambda_{2} \leq \ldots$, com $\lambda_{j} \rightarrow \infty$ quando $j \rightarrow \infty$, e a solução encontra-se no espaço de Hilbert $H$ gerado por estas funções.

Por exemplo,as equações de Navier-Stokes em duas dimensões podem ser expressas na forma do sistema (2.1), projetando-se as equações sobre o espaço de funções com divergência 
nula, onde $A u=-\nu \Delta u$ e $B(u, u)$ corresponde ao termo não linear $(u \cdot \nabla) u$. Outros exemplos de aplicações do método de Galerkin não Linear a equações que podem ser expressas nesta forma referem-se às equações de Burgers em uma dimensão [19] e [27], às equações de Kuramoto-Sivashinsky [36], às equações de Canh-Hillard [49], equações de Reação-Difusão [50], equação generalizada de Benjamin-Bona-Mahony [71], equação de Gizburg-Landau [58] e a equação da convecção de Benard em meio poroso [29]. Variações de implementações do método para as equações de Navier-Stokes foram sugeridas por Jabertau, Rosier and Temam ([33] e [34]) e Dubois, Jabertau e Temam [21]. Os resultados apresentados nestes artigos foram principalmente de testes envolvendo soluções exatas (analíticas), onde os autores recuperam os campos de pressão e velocidade. Estes testes indicam boa performance e aplicabilidade, sem considerar no entanto aplicações práticas. Descrevemos a seguir a idéia do método de Galerkin na forma (2.1). O método consiste na projeção das equações nas primeiras $N$ auto-funções (representada por $P_{N}$ ):

$$
\frac{\partial u_{N}}{\partial t}+P_{N} B\left(u_{N}, u_{N}\right)+A u_{N}=P_{N} f
$$

onde $u_{N}=P_{N} u$. O método de Galerkin não linear separa as equações em projeções $P_{N}$ (nos modos baixos) e em uma projeção complementar (nos modos altos) $Q_{d N}=P_{d N}-P_{N}$, onde $P_{d N}$ idealmente seria a identidade, mas na prática refere-se à projeção em um espaço de maior dimensão, geralmente $2 N$.

$$
\begin{aligned}
\frac{\partial y_{N}}{\partial t}+P_{N} B\left(y_{N}, y_{N}\right)+P_{N} B\left(y_{N}, z_{N}\right)+P_{N} B\left(z_{N}, y_{N}\right)+A y_{N} & =P_{N} f \\
\alpha \frac{\partial z_{N}}{\partial t}+Q_{d N} B\left(y_{N}, y_{N}\right)+A z_{N} & =Q_{d N} f
\end{aligned}
$$

onde $y_{N}=P_{N}(u)$ e $z_{N}=Q_{d N}(u)$ e $\alpha=0$ ou 1 .

O sistema é separado na equação dos modos baixos (2.3) e na equação dos modos altos (2.4). Na equação (2.3) leva-se em conta a interação não-linear dos modos altos com os baixos, enquanto que estes são desprezados na equação dos modos altos. Tomando $\alpha=0,(2.4)$ corresponde a uma equação diagnóstica que coincide com a variedade inercial aproximada estudada no Capítulo 1. A aplicabilidade de uma equação diagnóstica ou prognóstica é discutida em Debussche, Dubois e Temam [17]. Nesse artigo é implementado um método adaptativo multinível espacial e temporal para as equações de Navier-Stokes 
em duas dimensões, isto é, o nível de refinamento que define a separação das freqüências em altas ou baixas evolui no tempo, seguindo as escalas grandes e pequenas do movimento. Uma parte do espectro é congelada, assim como os termos de interação durante períodos curtos de tempo. Experimentos mostram que para $N$ suficientemente grande o espectro de energia decai exponencialmente e a equação (2.4) $\operatorname{com} \alpha=0$ é aplicável, enquanto que para $N$ muito pequeno o espectro de energia decai como uma potência das freqüências e $\alpha=1$ é usado na equação (2.4). Nesse artigo se fazem simulações com fluxos fisicamente mais relevantes, como o fluxo de Kolmogorov e fluxos com número de Reynolds grandes. Nos artigos citados são mencionadas as vantagens do método de Galerkin não linear sobre o método de Galerkin linear, no que concerne a eficiência, precisão e restrições de estabilidade.

A seguir, tratamos de esclarecer o porque desta superioridade, para isto trabalhamos com a equação de Burgers em uma dimensão com condições de contorno periódicas e forçante nula. Utilizaremos como teste para o esquema numérico um caso em que uma solução exata e completa desta equação é conhecida em função dos valores iniciais (ver [5] e [10]). A equação de Burgers é expressa por:

$$
\left\{\begin{array}{rl}
\frac{\partial u}{\partial t}+\frac{1}{2} \frac{\partial u^{2}}{\partial x}-\nu u_{x x} & =0, \quad x \in R, \quad t>0 \\
u(x, t) & =u(x+2 \pi, t)
\end{array} .\right.
$$

A solução exata que usaremos é definida pela função:

$$
u(x, t)=-2 \nu \frac{\phi_{x}(x-c t, t+1)}{\phi(x-c t, t+1)}
$$

onde

$$
\phi(x, t)=\sum_{n=-\infty}^{\infty} e^{\left.-(x-(2 n+1) \pi)^{2}\right) / 4 \nu t}
$$

A figura (2.1) mostra as condições iniciais para $c=4$ e vários valores da viscosidade $\nu(t=0$ em (2.6)). Para valores altos da viscosidade a função é bastante suave, e um gradiente forte acentua-se em $x=\pi / 2$ para a viscosidade decrescendo. A figura (2.2) apresenta a evolução da função para $\nu_{0}=0.2$, ela se desplaza com uma velocidade $c=4$ e gradualmente se difunde para um estado uniforme $(u=4)$. As simulações $\operatorname{com} \nu=0,2$ 


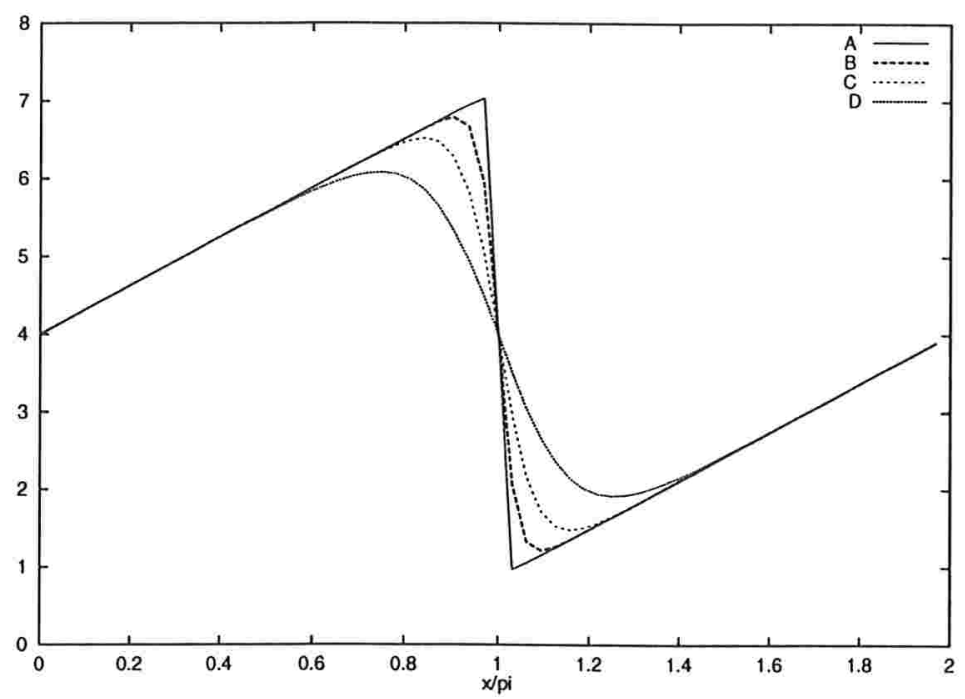

Figura 2.1: Eq. de Burgers: condição inicial $u(x, 0)$ A) $\nu=0,1, B) \nu=0,2, \quad C) \nu=0,4, \quad D) \nu=0,8$.

por exemplo, permitem distinguir a diferença de comportamento entre os métodos de Galerkin linear e não linear, pois um $N$ relativamente grande é necessário para uma boa aproximação da solução.

Discretizando a derivada temporal por um esquema de dois níveis no tempo $\left(t_{n}=n \Delta t\right)$

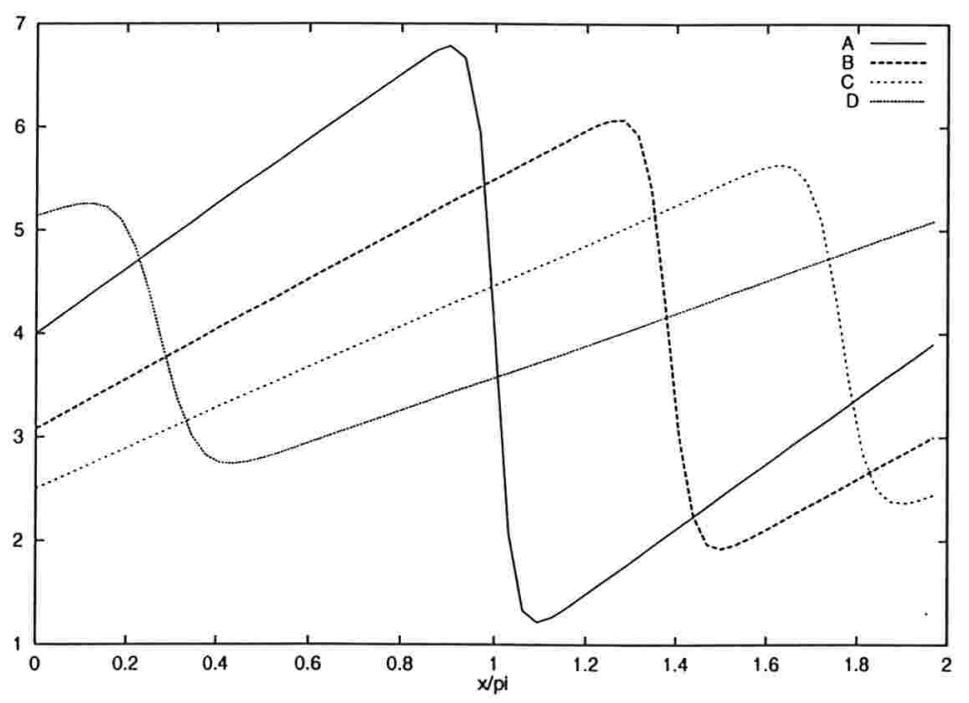

Figura 2.2: Eq. de Burgers: Perfil da velocidade $\left(\nu_{0}=0.2\right)$ A) $t=0, B) t=0,3$, C) $t=0,6, \quad$ D) $t=1$. 
e $\left.t_{n+1}=(n+1) \Delta t\right)$ com o termo linear avaliado no tempo $t_{n+1}$ e o termo não linear no tempo $t_{n}$, obtemos o método de Galerkin Linear com $N$ modos:

$$
\frac{u_{N}^{n+1}-u_{N}^{n}}{\Delta t}+\frac{1}{2} \frac{\partial}{\partial x} P_{N}\left(u_{N}^{n}\right)^{2}-\nu \frac{\partial^{2} u_{N}^{n+1}}{\partial x^{2}}=0
$$

enquanto que o método de Galerkin não linear com um total de $2 N$ modos é expresso pelo sistema:

$$
\begin{aligned}
\frac{y_{N}^{n+1}-y_{N}^{n}}{\Delta t}-\nu \frac{\partial^{2} y_{N}^{n+1}}{\partial x^{2}}+\frac{1}{2} \frac{\partial}{\partial x} P_{N}\left(\left(y_{N}^{n}\right)^{2}+2 y_{N}^{n} z_{N}^{n+1}\right) & =0 \\
-\nu \frac{\partial^{2} z_{N}^{n+1}}{\partial x^{2}}+\frac{1}{2} \frac{\partial}{\partial x} Q_{2 N}\left(y_{N}^{n}\right)^{2} & =0
\end{aligned}
$$

onde escolhemos $d=2$ e $\alpha=0$ na equação dos modos altos (2.4), e $y_{N}^{n}=P_{N} u\left(t_{n}\right)$, $z_{N}^{n}=Q_{2 N} u\left(t_{n}\right)$. A equação (2.9) representa a versão finita da variedade inercial para a equação de Burgers (tal variedade é idêntica a (2.9) -desde que $N$ seja suficientemente grande- exceto que é usada a projeção complementar de dimensão infinita $Q=I-P_{N}$ em vez da projeção finita $\left.Q_{2 N}\right)$.

Ainda que redundante, escrevemos a equação pelo método de Galerkin linear com $2 N$ modos:

$$
\frac{u_{2 N}^{n+1}-u_{2 N}^{n}}{\Delta t}+\frac{1}{2} \frac{\partial}{\partial x} P_{2 N}\left(u_{2 N}^{n}\right)^{2}-\nu \frac{\partial^{2} u_{2 N}^{n+1}}{\partial x^{2}}=0
$$

isto porque desejamos comparar o método de Galerkin não linear dado pelo sistema (2.8)(2.9) com o método de Galerkin linear (2.7) e com o método de Galerkin linear (2.10). Intuitivamente espera-se que a solução de (2.10) por conter mais informação seja mais precisa que a solução do sistema (2.8)-(2.9), e esta per sua vez seja mais precisa que a solução de (2.7). Quão próxima está a solução do método de Galerkin não linear das soluções dos métodos de Galerkin linear apresentadas e qual é a relação da eficiência destes métodos são questões simultâneas a estudar.

Temos as seguintes representações:

$$
u_{2 N}(x, t)=\sum_{k=-N}^{N} \hat{u}_{k}(t) e^{i k x}, y_{N}(x, t)=\sum_{k=-N / 2}^{N / 2} \hat{y}_{k}(t) e^{i k x}, z_{N}(x, t)=\sum_{|k|=N / 2+1}^{2 N} \hat{z}_{k}(t) e^{i k x},
$$


onde os coeficientes $\hat{u}_{k}(t), \quad \hat{y}_{k}(t)$ e $\hat{z}_{k}(t)$ são chamados de coeficientes espectrais. A substituição de (2.11) na equação (2.10) resulta em:

$$
\frac{\hat{u}_{k}^{n+1}-\hat{u}_{k}^{n}}{\Delta t}+\nu k^{2} \hat{u}_{k}^{n+1}+r_{k}=0, \quad k=-N, \ldots, N
$$

onde $r_{k}$ são os coeficientes espectrais de $\frac{1}{2} \frac{\partial}{\partial x} P_{2 N}\left(u_{2 N}^{n}\right)^{2}$, e a substituição no sistema (2.8)(2.9) leva ao seguinte sistema:

$$
\begin{aligned}
\frac{\hat{y}_{k}^{n+1}-\hat{y}_{k}^{n}}{\Delta t}+\nu k^{2} \hat{y}_{k}^{n+1}+s_{1, k} & =0, \quad k=-N / 2, \ldots, N / 2 \\
\nu k^{2} \hat{z}_{k}^{n+1}+s_{2, k} & =0, \quad|k|=N / 2+1, \ldots, N
\end{aligned}
$$

onde, $s_{1, k}$ e $s_{2, k}$ são os coeficientes espectrais de $\frac{1}{2} \frac{\partial}{\partial x} P_{N}\left(\left(y_{N}^{n}\right)^{2}+2 y_{N}^{n} z_{N}^{n+1}\right)$ e $\frac{1}{2} \frac{\partial}{\partial x} Q_{2 N}\left(y_{N}^{n}\right)^{2}$ respectivamente. A solução destes sistemas é feita usando o chamado método pseudoespectral, ou método das Transformadas (Orzag, Machenhauer). Os termos não lineares são avaliados em pontos de uma malha discreta no domínio (o espaço das funções na malha será chamado espaço físico), seus coeficientes espectrais podem ser obtidos através da transformação da função no espaço físico à sua expansão em coeficientes espectrais (denotemos este espaço de funções por espaço espectral). Um modo eficiente de fazer esta transformação de um espaço ao outro é usar a Transformada Rapida de Fourier (FFT: Fast Fourier Transform) definida por Cooley e Tukey [12] em 1965. Para isto as funções em (2.11) são avaliadas nos pontos $x_{j}^{M}=\frac{2 \pi(j-1)}{M}, \quad 1 \leq j \leq M$, enquanto os coeficientes espectrais são avaliados usando a transformada inversa:

$\hat{u}_{k}=\frac{1}{2 N} \sum_{j=1}^{2 N} u_{2 N}\left(x_{j}^{2 N}\right) e^{-i k x_{j}^{2 N}}, \quad \hat{y}_{k}=\frac{1}{N} \sum_{j=1}^{N} y_{N}\left(x_{j}^{N}\right) e^{-i k x_{j}^{N}}, \quad \hat{z}_{k}=\frac{1}{2 N} \sum_{j=1}^{2 N} z_{2 N}\left(x_{j}^{2 N}\right) e^{-i k x_{j}^{2 N}}$.

Há uma correspondência biunívoca entre os coeficientes espectrais e os valores na malha de uma função $u_{N}$, desde que a malha contenha tantos pontos quanto os coeficientes. No entanto, um termo quadrático como $\left(u_{N}\right)^{2}$ não pertence mais ao mesmo espaço (na expansão espectral possui o dobro de termos). Como os modos adicionais $\mathrm{v}_{k+N}=e^{i(k+N) x_{j}^{2 N}}, \quad k=1, \cdots, N$ coincidem com os modos $\overline{\mathrm{v}}_{N-k}$ em uma malha de $2 N$ pontos $\left(x_{j}^{2 N}=2 \pi \frac{(j-1)}{2 N}\right)$, se esta for usada na transformada de Fourier, os coeficientes espectrais não serão corretamente avaliados. Esta reflexão de modos altos em modos baixos 
é conhecida como 'aliasing'na literatura e se constitui em uma fonte de instabilidade para os métodos espectrais (ver [3], [35], [54], [55]. Para se evitar aliasing dos termos não lineares é necessário avaliar em malha com pelo menos $3 \mathrm{~N}$ pontos. Com efeito, o aliasing nos modos $\mathrm{v}_{k+3 N / 2}, k=1, \cdots, N / 2$ revertem sobre os modos $\overline{\mathrm{v}}_{-k+3 N / 2}, k=1, \cdots, N / 2$ (ou seja sobre eles mesmos), deixando os coeficientes espectrais $\hat{u}_{k}^{n}, k= \pm 1, \cdots, \pm N$ livres de aliasing (isto é, eles são avaliados corretamente). Logo para avaliar corretamente em cada passo no tempo o termo não linear em (2.12) precisamos de duas FFTs com tamanho $3 N$, uma (direta) para avaliar os valores da função nos pontos da malha (na qual é executado o produto) e outra (inversa) a seguir para avaliar corretamente os coeficientes espectrais de $r_{k}$.

No método de Galerkin não linear os termos não lineares do sistema (2.13)-(2.14) são $\left(y_{N}^{n}\right)^{2}, \quad y_{N}^{n} z_{N}^{n+1}$. Usando como antes FFTs de tamanho $3 N$ o termo $\left(y_{N}^{n}\right)^{2}$ tem modos $\mathrm{v}_{k}, \quad k=-N, \cdots N$, porem $P_{N}\left(y_{N}^{n}\right)^{2}$ em (2.13) e $Q_{2 N}\left(y_{N}^{n}\right)^{2}$ em (2.14) são avaliados corretamente, enquanto que o termo $y_{N}^{n} z_{N}^{n+1}$ tem modos $\mathrm{v}_{k}, \quad k=-3 N / 2, \cdots 3 N / 2$, logo $P_{N}\left(y_{N}^{n} z_{N}^{n+1}\right)$ em (2.13) também é avaliado corretamente. No total são necessárias 4 FFTs de tamanho $3 N$ para avaliar em cada iteração no tempo os termos não lineares em (2.13)(2.14): duas para avaliar $\left(y_{N}^{n}\right)$ no espaço fisico e duas para avaliar $\left(z_{N}^{n}\right)$. Com esta escolha o método de Galerkin não linear precisaria do dobro de trabalho para avaliar os termos não lineares que o método de Galerkin linear, o que resultaria num método pouco competitivo. A chave para tornar competitivo este método quando comparado com o método de Galerkin linear está na implementação dos termos não lineares. Observando as contas anteriores vemos que avaliar os termos não lineares com FFTs de tamanho $2 N$ é suficiente para evitar os efeitos de aliasing; pois $\left(y_{N}^{n}\right)^{2}$ segue sendo avaliado corretamente, enquanto o aliasing nos modos $\mathrm{v}_{k}, \quad k= \pm(N+1), \cdots \pm 3 N / 2$ do termo $y_{N}^{n} z_{N}^{n+1}$ é revertido nos modos $\mathrm{v}_{k}, \quad k= \pm(N / 2+1), \cdots \pm N$, não afetando a projeção $P_{N}\left(y_{N}^{n} z_{N}^{n+1}\right)$, que é avaliada corretamente. Logo o custo computacional por iteração no tempo dos termos não lineares de (2.13)-(2.14) é de 4 FFTs de tamanho $2 N$.

Considerando que o custo computacional se concentra na avaliação das FFTs e conhecendo que este custo é da ordem de $N \log N$ para FFTs de tamanho $N$, temos que por iteração no tempo o método de Galerkin linear (2.12) é da ordem de $6 N \log (3 N)$, enquan- 


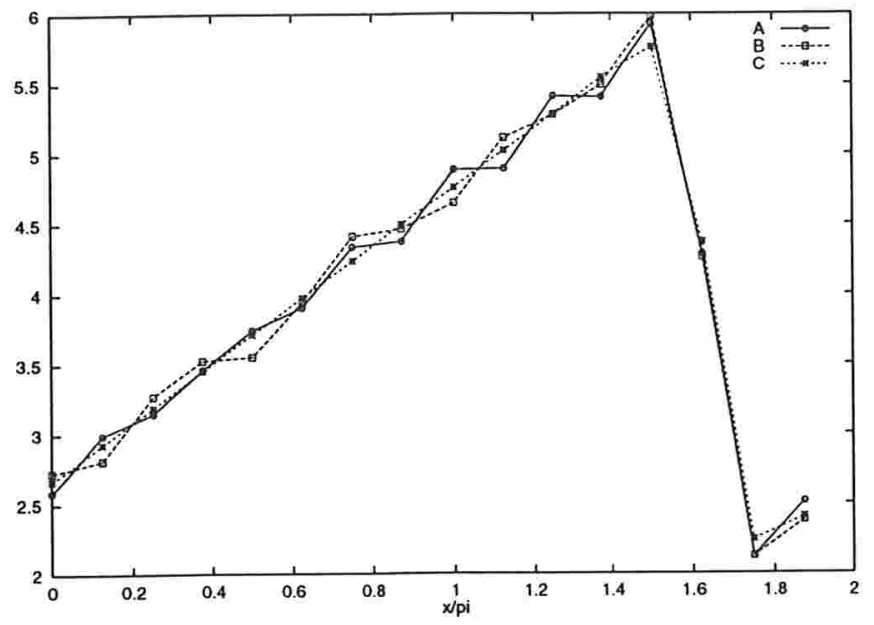

Figura 2.3: Eq. de Burgers: GL e GNL com 16 modos
A) GL: $P_{16}$
B) GNL: $P_{8}+Q_{16}$
C) Exata

to que o método de Galerkin não linear (2.13)-(2.14) é da ordem $8 N \log (2 N)$; isto é ainda aproximadamente $33 \%$ mais custoso!. Parece contraditório apostar neste método para a equação de Burgers, mas os experimentos numéricos feitos aqui mostram que o método de Galerkin não linear pode ser competitivo.

Nas Figuras (2.3), (2.4)e (2.5) são comparados os métodos de Galerkin não linear com

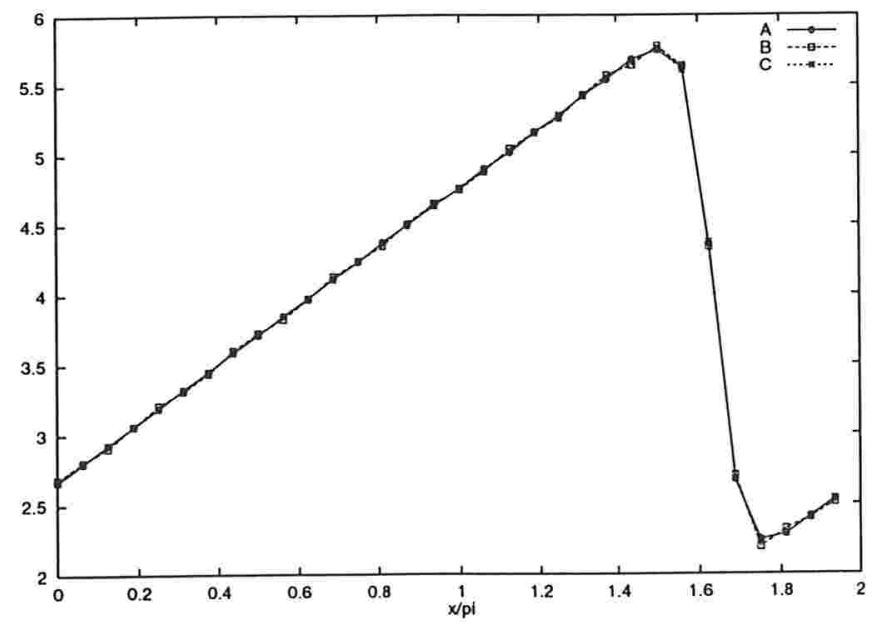

Figura 2.4: Eq. de Burgers: GL e GNL com 32 modos
A) GL: $P_{32}$
B) GNL: $P_{16}+Q_{32}$
C) Exata 


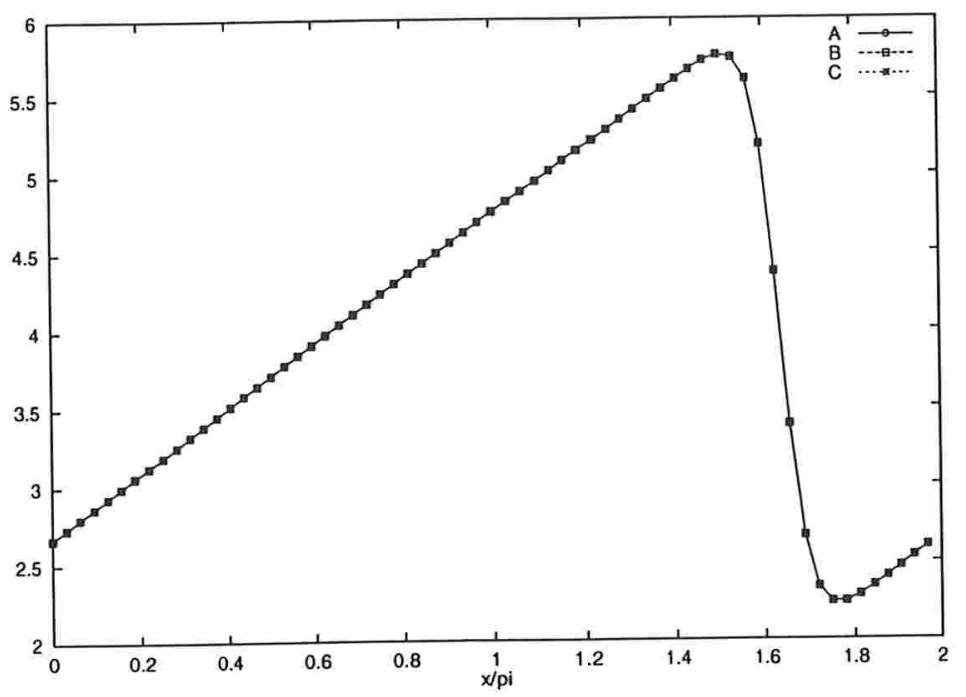

Figura 2.5: Eq. de Burgers: GL e GNL com 64 modos

A) GL: $P_{64}$; B) GNL: $P_{32}+Q_{64}$ C) Exata

Galerkin linear e a solução exata para um número total de 16, 32 e 64 modos usando uma viscosidade $\nu=0,2$, um passo de tempo $\Delta t=10^{-4}$ no instante $t=0,5$ equivalente a 5000 iterações no tempo. Os resultados mostram que o método de Galerkin linear obtido pela projeção $P_{2 N}$ é mais preciso que o método de Galerkin não linear obtido pela projeção $P_{N}+Q_{2 N}$.

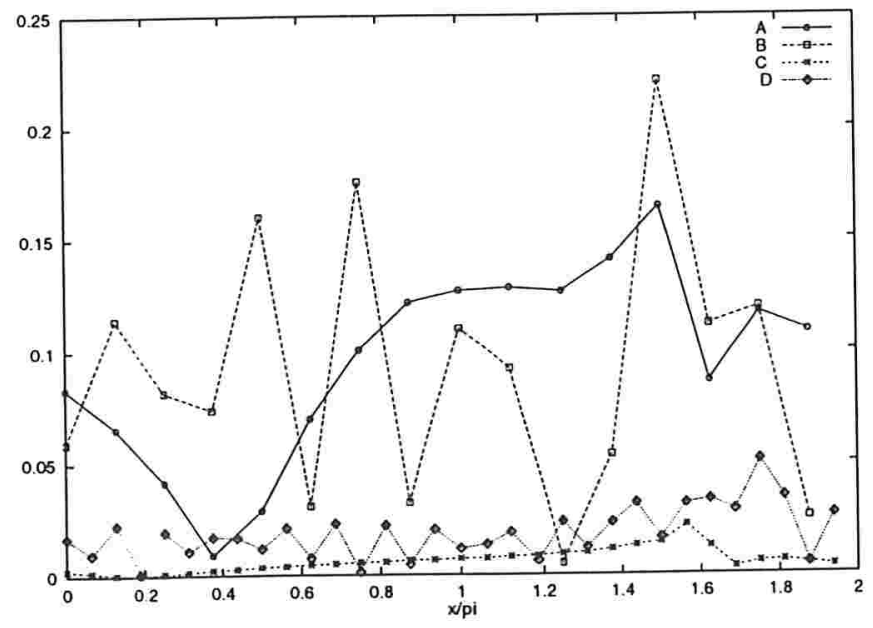

Figura 2.6: Eq. de Burgers: Erro de GL e GNL com 16 e 32 modos Erro $=\mid f\left(x_{j}\right)-$ exata $\left(x_{j}\right) \mid:$ A) GL: $P_{16}$; B) GNL: $P_{8}+Q_{16}$ C) GL: $P_{32}$; D) GNL: $P_{16}+Q_{32}$ 


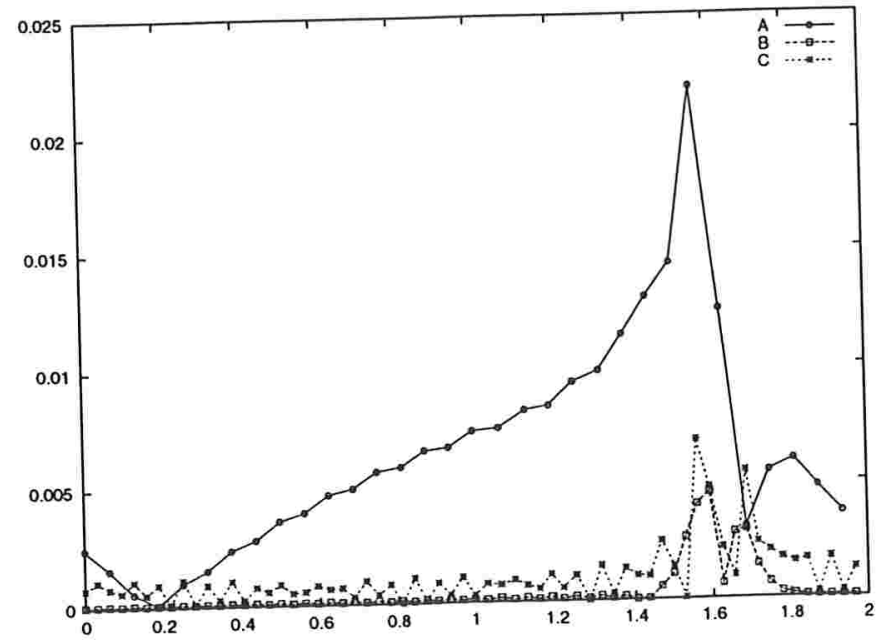

Figura 2.7: Eq. de Burgers: Erro de GL e GNL com 32 e 64 modos Erro $=\left|f\left(x_{j}\right)-\operatorname{exata}\left(x_{j}\right)\right|:$ A) GL: $P_{32}$; B) GL: $P_{64}$; C) GNL: $P_{32}+Q_{64}$

Para ver quão perto são as soluções de um e outro método comparamos o erro pontual obtido pelo método de Galerkin linear pela projeção $P_{N}$ e $P_{2 N}$ com o método de Galerkin não linear obtido pela projeção $P_{N}+Q_{2 N}$, os resultados são apresentados nas Figuras (2.6) e (2.7). Pode-se apreciar a característica que consideramos fundamental do método de Galerkin não linear obtido pela projeção $P_{N}+Q_{2 N}$ : o erro neste caso se situa entre os erros do método de Galerkin linear obtido pelas projeções $P_{N}$ e $P_{2 N}$, estando porém

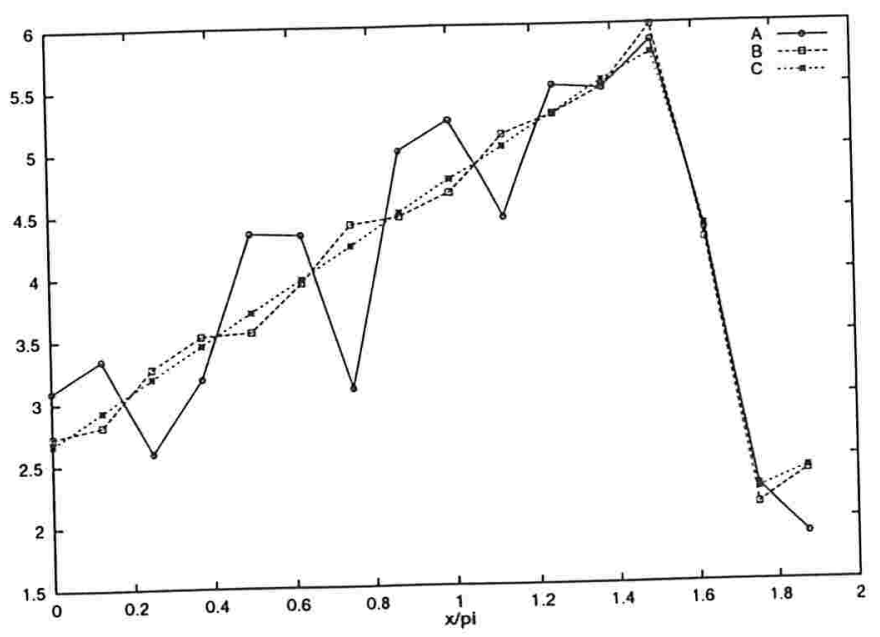

Figura 2.8: Eq. de Burgers (modos altos desligados): GL e GNL com 16 modos A) GNL: $P_{8}+Q_{16}$ (Eq. (2.14) desligada); B) GNL: $P_{8}+Q_{16}$; C) Exata. 


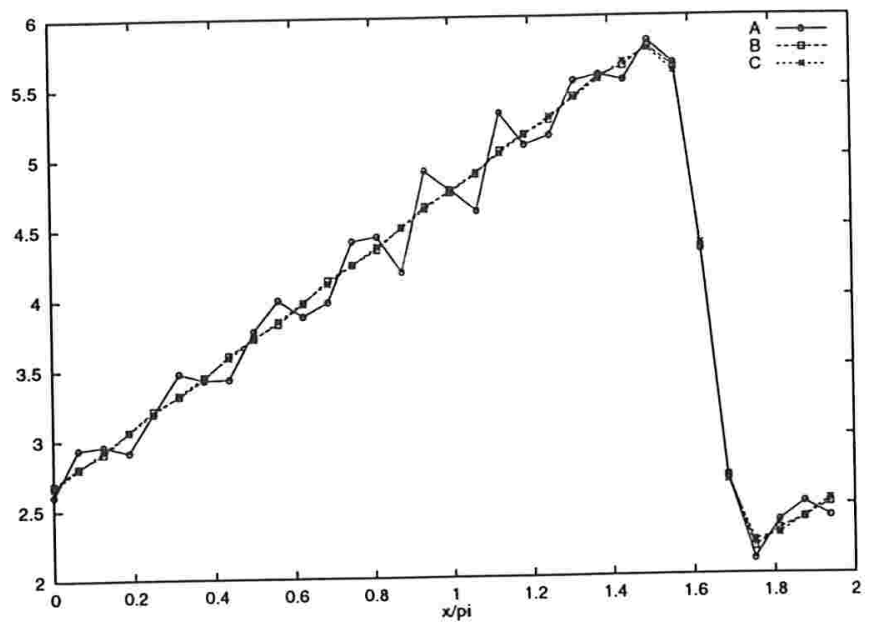

Figura 2.9: Eq. de Burgers (modos altos desligados): GL e GNL com 32 modos A) GNL: $P_{16}+Q_{32}$ (Eq.(2.14) desligada); B) GNL: $P_{16}+Q_{32}$; C) Exata.

bem mais próximo ao de $P_{2 N}$. Resultados similares (não apresentados aqui) são observados para outros valores dos parâmetros. Para perceber a importância da equação dos modos altos (2.14) esta é desligada, sendo o método de Galerkin não linear avaliado só com a equação dos modos baixos (2.13) e os dados inicias $y_{N}^{0}$ e $z_{N}^{0}$. Os resultados são apresentados nas Figuras (2.8) e (2.9). Observa-se que $z_{N}^{n+1}$ atua como uma correção de $y_{N}^{n+1}$ (tal correção é da ordem da diferença entre $u_{2 N}^{n+1}$ e $u_{N}^{n+1}$ ). Seguindo os experimentos

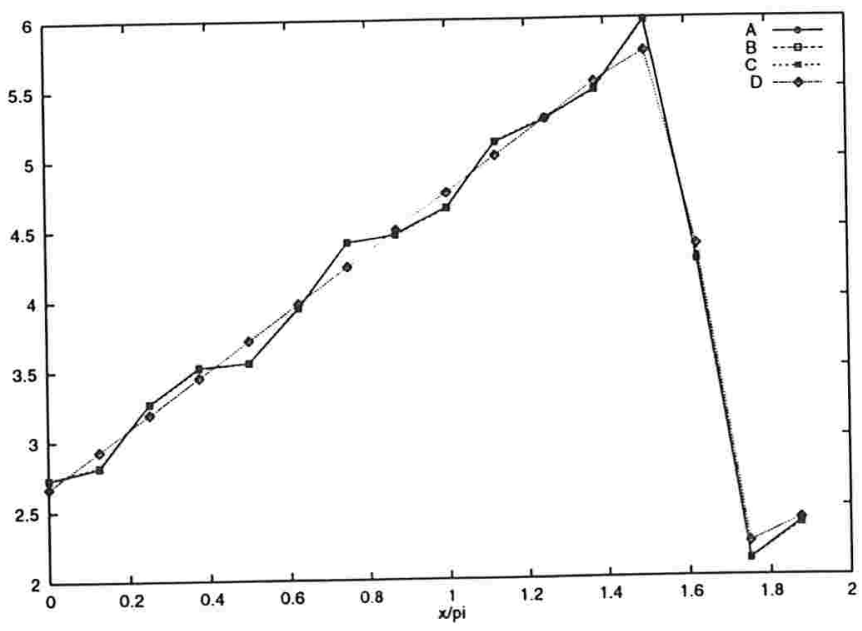

Figura 2.10: Eq. de Burgers (modos altos congelados): GNL com 16 modos Eq. (2.14) avaliada: A) sempre; B) cada 10 iterações; C) cada 50 iterações; D) Exata. 


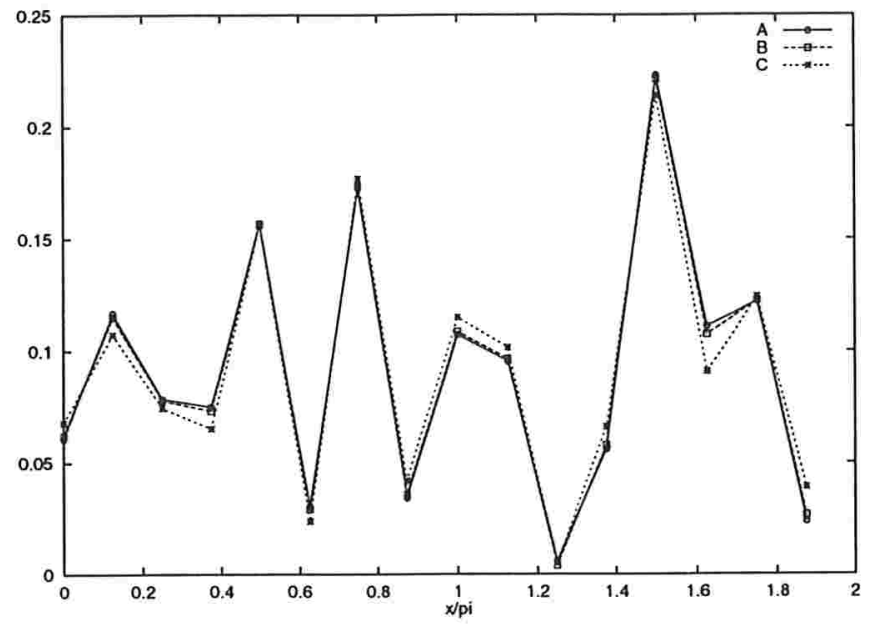

Figura 2.11: Eq. de Burgers (modos altos congelados): Erro de GNL com 16 modos Erro $=\mid G N L\left(P_{8}+Q_{16}\right)-$ exata $\mid$ com a Eq. (2.14) avaliada: A) sempre; B) c/ iter.; C) c/ 50 iter.

mencionados em [19], [27], [33] e [34] acionamos a equação dos modos altos somente a cada $x$ iterações (onde $x$ é um dado número), mantendo $z_{N}$ congelado nos passos intermediários. Nas Figuras (2.10), (2.12) e (2.14) são comparados o método de Galerkin não linear (com $\mathrm{N}=8,16$ e 32 modos para cada equação dos modos altos e baixos) com os modos altos congelados por 10 e 50 iterações, enquanto nas Figuras (2.11), (2.13) e (2.15) se apresentam os erros com respeito à solução exata. Os resultados mostram que com 10

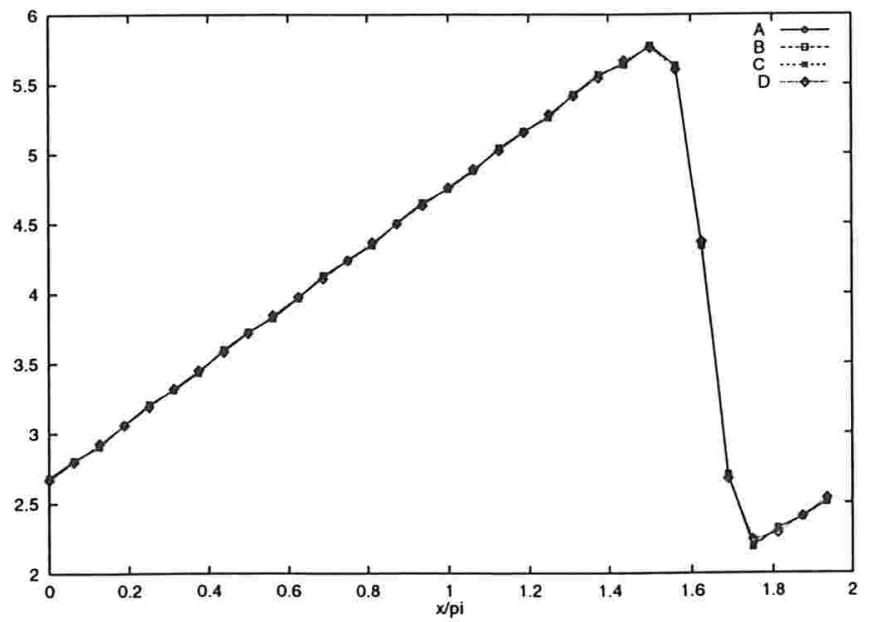

Figura 2.12: Eq. de Burgers (modos altos congelados): GNL com 32 modos Eq. (2.14) avaliada : A)sempre; B) cada 10 iter. ; C) cada 50 iter. ; D) Exata. 


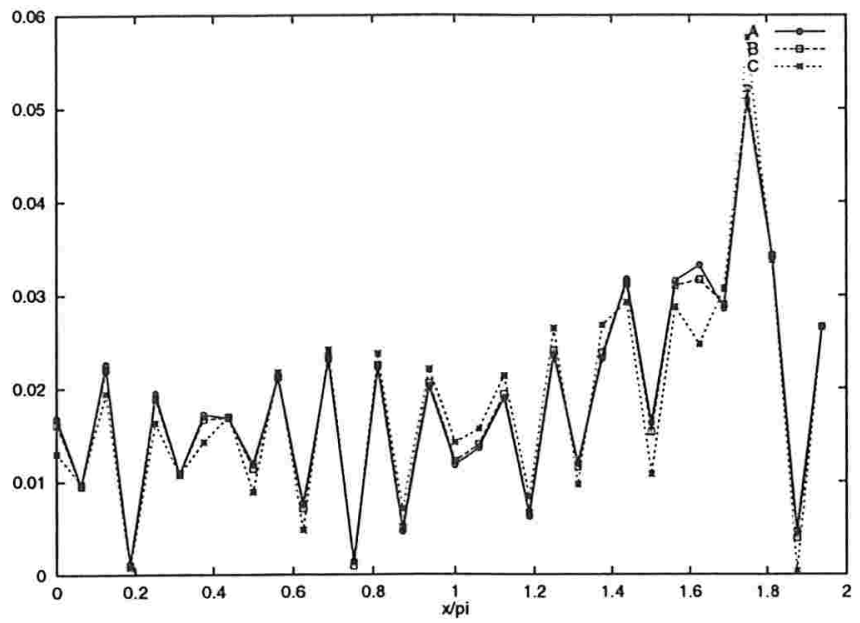

Figura 2.13: Eq. de Burgers (modos altos congelados): Erro de GNL com 32 modos Erro $=\mid G N L\left(P_{16}+Q_{32}\right)-$ exata $\mid$ com a Eq. (2.14) avaliada: A) sempre: B) c/ 10 iter.; C) c/ 50 iter.

ou 50 iterações tem-se pouca perda de precisão com respeito ao método de Galerkin não linear sem desligar os modos altos. Em conclusão, pode-se usar o método de Galerkin não linear com a equação dos modos altos ativada só de quando em quando (a cada $l$ passos) sem haver perda significativa de precisão. Desta forma, o custo computacional se reduz aproximadamente pela metade, tornando o método de Galerkin não linear aproximadamente $33 \%$ mais barato que o método de Galerkin linear obtido pela projeção $P_{2 N}$.

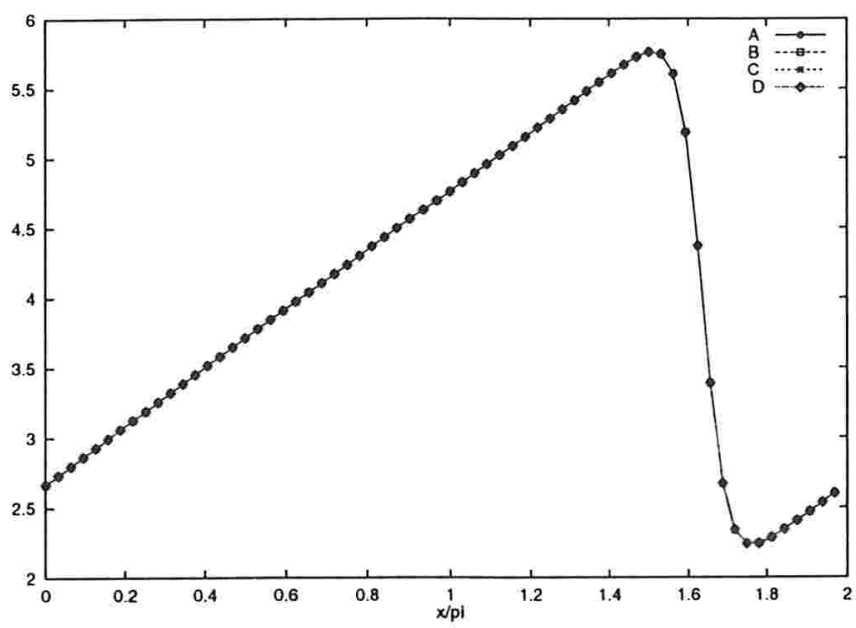

Figura 2.14: Eq. de Burgers (modos altos congelados): GNL com 64 modos Eq. (2.14) avaliada : A)sempre; B)cada 10 iter. ; C)cada 50 iter. ; D) Exata. 


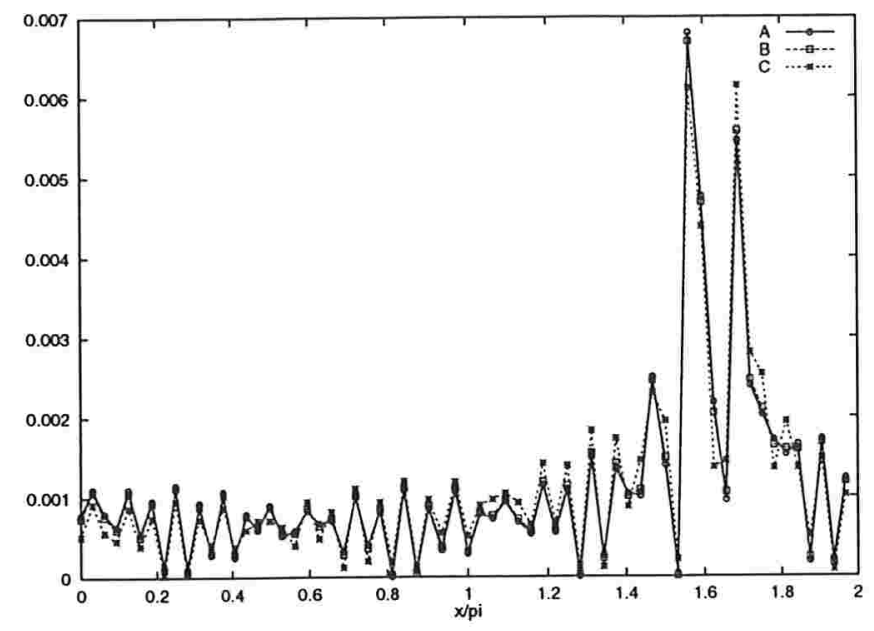

Figura 2.15: Eq. de Burgers (modos altos congelados): Erro de GNL com 64 modos Erro $=\mid G N L\left(P_{32}+Q_{64}\right)-$ exata $\mid$ com a Eq. (2.14) avaliada: A) sempre; B) c/ 10 iter.; C) c/ 50 iter.

Isto torna o método de Galerkin não linear competitivo com o método de Galerkin linear para a equação de Burgers em uma dimensão, em situações em que a variação temporal dos modos altos ocorre mais lentamente que a dos modos baixos, permitindo seu congelamento, como neste exemplo.

Um outro ponto a analisar nos experimentos numéricos refere-se à estabilidade dos esquemas. Nas Tabelas (2.1)-(2.3) apresentamos os valores máximos (obtidos numericamente) do passo do tempo para que os esquemas sejam estáveis. Observa-se que o método de Galerkin não linear obtido com a projeção $P_{N}+Q_{2 N}$ admite maiores valores do passo

\begin{tabular}{|c|c|c|c|}
\hline $2 \mathrm{~N}$ & $\Delta t$ (GNL: $\mathrm{N}+\mathrm{N}$ modos) & $\Delta t$ (GL : 2N modos) & $\begin{array}{c}\Delta t \text { (GNL }: \mathrm{N}+\mathrm{N} \text { modos) } \\
\text { cada } 100 \text { iterações }\end{array}$ \\
\hline 16 & 0,017 & 0,0055 & 0,0055 \\
\hline 32 & 0,019 & 0,0047 & 0,0058 \\
\hline 64 & 0,013 & 0,0041 & 0,0047 \\
\hline 128 & 0,0053 & 0,0039 & 0,0040 \\
\hline 256 & 0,0043 & 0,0037 & 0,0040 \\
\hline 512 & 0,0037 & 0,0037 & 0,0037 \\
\hline
\end{tabular}

Tabela 2.1: Valores limites $\Delta t$ para a estabilidade $(\nu=0,06)$ 


\begin{tabular}{|c|c|c|c|}
\hline $2 \mathrm{~N}$ & $\Delta t$ (GNL: N+N modos) & $\Delta t$ (GL : 2N modos) & $\begin{array}{c}\Delta t \text { (GNL }: \mathrm{N}+\mathrm{N} \text { modos) } \\
\text { cada } 100 \text { iterações }\end{array}$ \\
\hline 16 & 0,0255 & 0,0088 & 0,0107 \\
\hline 32 & 0,029 & 0,0077 & 0,0088 \\
\hline 64 & 0,011 & 0,00706 & 0,0077 \\
\hline 128 & 0,0080 & 0,00644 & 0,00702 \\
\hline 256 & 0,0065 & 0,00633 & 0,00643 \\
\hline 512 & 0,00633 & 0,00633 & 0,00633 \\
\hline
\end{tabular}

Tabela 2.2: Valores limites $\Delta t$ para a estabilidade $(\nu=0,1)$

de tempo $\Delta t$ que os método de Galerkin linear obtidos com as projeções $P_{2 N}$ e $P_{N}$, e no caso de congelar os modos altos a cada 100 iterações o método de Galerkin não linear tem aproximadamente a mesma restrição de estabilidade que o método de Galerkin linear obtido com a projeção $P_{N}$. Uma análise detalhada da estabilidade, convergência e resultados numéricos com outros métodos de integração no tempo para a equação de Burgers está em preparação [8].

Em [19], [27] uma outra implementação para a equação de Burgers 1D é estudada, usando um método espectral de colocação, onde os termos $y_{N}$ e $z_{N}$ são avaliados com FFts de tamanho $\mathrm{N}$ ( em nossa implementação usamos FFts de tamanho $2 \mathrm{~N}$ ). O custo computacional para o método de Galerkin não linear obtido pela projeção $P_{N}+Q_{2 N}$ é de aproximadamente $8 N \log N$, uma ligeira melhora com relação ao método apresentado aqui $(8 N \log (2 N))$. Esta outra implementação é mais elaborada e os autores não apre-

\begin{tabular}{|c|c|c|c|}
\hline $2 \mathrm{~N}$ & $\Delta t$ (GNL: N+N modos) & $\Delta t$ (GL : 2N modos) & $\begin{array}{c}\Delta t \text { (GNL : N+N modos) } \\
\text { cada } 100 \text { iterações }\end{array}$ \\
\hline 16 & 0,063 & 0,017 & 0,018 \\
\hline 32 & 0,025 & 0,015 & 0,016 \\
\hline 64 & 0,016 & 0,0134 & 0,015 \\
\hline 128 & 0,0135 & 0,01336 & 0,0134 \\
\hline 256 & 0,01336 & 0,01336 & 0,01336 \\
\hline 512 & 0,01336 & 0,01336 & 0,01336 \\
\hline
\end{tabular}

Tabela 2.3: Valores limites $\Delta t$ para a estabilidade $(\nu=0,2)$ 
sentam evidências numéricas dos efeitos no custo computacional desta abordagem. A implementação que escolhemos não apenas é simples mas admite uma extensão eficiente para as equações de Água Rasa com condições de contorno periódicas em duas dimensões (estudada na próxima seção). Mostraremos que o método de Galerkin não linear para estas equações tem menor custo computacional que o método de Galerkin linear, mesmo sem congelar a equação dos modos altos!. 


\subsection{Métodos de Galerkin para as equações de Água Rasa}

No capítulo 1, estudou-se a aplicabilidade da teoria das variedades inerciais aproximadas às equações de água rasa (1.7) e (1.8). Aqui aplicamos os métodos de Galerkin linear e não linear para estas equações. No método de Galerkin linear aplica-se um truncamento direto sobre as primeiras $N \times N$ autofunções, e no caso do Galerkin não linear usa-se um truncamento mais elaborado, baseado numa partição em modos baixos e altos (a ser explicada mais adiante).

Métodos de Galerkin não linear foram propostos para as equações incompressíveis e viscosas de Navier Stokes com condições de contorno periódicas (ver [21], [34], [17], [48]). As funções base (autofunções do operador de Stokes) são divididas em dois conjuntos (os modos baixos e altos) e as equações são projetadas sobre estes conjuntos, separando-se as equações para os modos baixos e altos. A interação entre os modos altos nos termos nãolineares é desconsiderada na equação dos modos baixos, e no caso da equação dos modos altos só é considerada a interação não linear entre modos baixos; levando a um sistema que pode ser resolvido para os modos altos, e a seguir para os modos baixos. Debussche [17] usa um método multinível no tempo, estudando escalas (espaciais e temporais) nas quais os modos altos podem ser congelados. O método de Runge-Kutta de ordem 4 é usado na discretização temporal, e os resultados apresentados mostram um ganho em eficiência computacional de 30 a 45 por cento do método de Galerkin não linear (com os modos altos congelados) com respeito ao método de Galerkin linear.

As equações de água rasa (1.7) e (1.8) diferem das equações de Navier Stokes incompressíveis por não apresentarem divergente nulo, levando a uma equação de massa com dependencia temporal explícita. Além disso, as equações de momento, incluem o termo de Coriolis devido aos efeitos de rotação da terra. A seguir apresentamos em detalhe nossa proposta de um método Galerkin não linear para as equações de água rasa. Comparamos o método de Galerkin não-linear proposto ao método de Galerkin linear, em relação a aspectos de precisão, exatidão e estabilidade. O aspecto crucial do método está na forma de implementação dos termos não lineares (usando transformadas rápidas de Fourier) e suas projeções nos modos altos e baixos. Nosso método utiliza uma discreti- 
zação semi-implícita, tratando implicitamente os termos do gradiente do geopotencial e a parte linear do divergente de massa, além dos termos viscosos. Desta forma evita-se fortes restrições de estabilidade (tipo CFL) que seriam causadas pelas ondas de gravidade. O tratamento explícito dos termos não lineares ainda garante a obtenção de um sistema que pode ser resolvido eficientemente (Métodos espectrais foram originalmente propostos por Robert para modelos atmosféricos). Investigamos duas formulações para o método de Galerkin não linear, uma avaliando os modos altos de forma diagnóstica e outra de forma prognóstica. Nosso método de Galerkin não linear pode em pricipio ser aplicado para as equações de água rasa na esfera, dependendo no entanto de uma decomposição eficiente dos polinomios de Legendre em modos altos e baixos (primeiros estudos sobre estes polinômios e métodos de Galerkin aparecem em [62] e [63]).

As equações normalmente utilizadas para modelos atmosféricos são de natureza hiperbólica, não incluindo explicítamente os termos viscosos. Aqui estudamos a implementação numérica das equações na sua forma introduzida por Lorenz [47], com a adicão de termos viscosos e uma forçante na vertical, além de condições de contorno periódicas nos campos da velocidade e no geopotencial.

\subsubsection{Descrição do método linear}

As equações de água rasa na forma adimensionalizada (incluindo termos viscosos e forçante são formuladas como:

$$
\begin{aligned}
\left.\frac{\partial u}{\partial t}+u u_{x}+v u_{y}-v+z_{x}-\nu_{0}\right\lrcorner u & =0 \\
\frac{\partial v}{\partial t}+u v_{x}+v v_{y}+u+z_{y}-\nu_{0} \Delta v & =0 \\
\frac{\partial z}{\partial t}+g_{0}\left(u_{x}+v_{y}\right)+z\left(u_{x}+v_{y}\right)+u z_{x}+v z_{y}-\kappa_{0} \Delta z & =F
\end{aligned}
$$

onde, $u$ e $v$ são as componentes da velocidade, $z$ é o geopotencial, $F$ é a forçante, $\nu_{0}$ e $g_{0}$ são constantes adimensionalizadas correspondentes as constantes $\nu$ e $g$ respectivamente (ver página 19 para uma descripção destas constantes). 
Usamos um método semi-implícito com 3 níveis no tempo para o sistema (2.16)-(2.18) com os termos dissipativos e os termos correspondentes às ondas de gravidade (Veja Pedlosky [57]) tratados implicitamente como uma média temporal num intervalo de dois passos no tempos (evitando sérias restrições de estabilidade), enquanto os termos não lineares (ligados às ondas de Rossby) são mantidos explícitos para que os sistemas discretos correspondentes sejam eficientemente inversíveis. O termo de Coriolis (sendo constante), também é mantido implícito (pois ainda é simples inverter o sistema discreto resultante). Esta discretização temporal de segunda ordem é resumida no esquema seguinte:

\begin{tabular}{|c|c|c|c|c|}
\hline Termo & $\frac{\partial \theta}{\partial t}$ & Linear (L) & Não linear (NL) & Forçante (F) \\
\hline Discretização & $\frac{\theta^{n+1}-\theta^{n-1}}{2 \Delta t}$ & $\frac{1}{2}\left(L^{n+1}+L^{n-1}\right)$ & $(N L)^{n}$ & $F$ \\
\hline
\end{tabular}

isto é, as derivadas temporais são aproximadas usando diferença central, toma-se a média dos termos lineares nos tempos $t_{n+1}=(n+1) \Delta t$ e $t_{n-1}=(n-1) \Delta t$, os termos não lineares são avaliados no tempo $t_{n}=n \Delta t$, e a forçante $F$ independe do tempo. Estas aproximações levam ao sistema:

$$
\begin{aligned}
& u^{n+1}-\Delta t v^{n+1}+\Delta t z_{x}^{n+1}-\nu_{0} \Delta t \Delta u^{n+1}=r_{1 a}^{n-1}+r_{1 b}^{n} \\
& v^{n+1}+\Delta t u^{n+1}+\Delta t z_{y}^{n+1}-\nu_{0} \Delta t \Delta v^{n+1}=r_{2 a}^{n-1}+r_{2 b}^{n} \\
& z^{n+1}+g_{0} \Delta t\left(u_{x}^{n+1}+v_{y}^{n+1}\right)-\kappa_{0} \Delta t \Delta z^{n+1}=r_{3 a}^{n-1}+r_{3 b}^{n}
\end{aligned}
$$

onde $r_{i a}^{n-1}$ são os termos lineares no tempo $t_{n-1}$ e $r_{i b}^{n}$ são os termos não lineares em $t_{n}$, para $i=1,2,3$. Estes termos são definidos por:

$$
\begin{aligned}
r_{1 a}^{n-1} & =u^{n-1}+\Delta t v^{n-1}-\Delta t z_{x}^{n-1}+\nu_{0} \Delta t \Delta u^{n-1} \\
r_{2 a}^{n-1} & =v^{n-1}-\Delta t u^{n-1}-\Delta t z_{y}^{n-1}+\nu_{0} \Delta t \Delta v^{n-1} \\
r_{3 a}^{n-1} & =z^{n-1}-\Delta t g_{0}\left(u_{x}^{n-1}+v_{y}^{n-1}\right)+\kappa_{0} \Delta t \Delta z^{n-1} \\
r_{1 b}^{n} & =-2 \Delta t\left(u^{n} u_{x}^{n}+v^{n} u_{y}^{n}\right) \\
r_{2 b}^{n} & =-2 \Delta t\left(u^{n} v_{x}^{n}+v^{n} v_{y}^{n}\right) \\
r_{3 b}^{n} & =-2 \Delta t\left(\left(z^{n} u^{n}\right)_{x}+\left(z_{n} v_{n}\right)_{y}+F\right)
\end{aligned}
$$


Agora associamos ao sistema (2.19)-(2.21) o método espectral para a discretização no espaço. Devido às condições de contorno periódicas em ambas direções, aproximamos os campos da velocidade e o geopotencial pela sua representação em série de Fourier.

Se $u, v$ e $z$ são funções de $\mathbf{H}_{\text {per }}$, elas podem ser aproximadas pela sua representação em série de Fourier, onde as funções base adequadas são as auto-funções $\mathrm{V}_{l k}=e^{i(l x+k y)}$ do operador $-\Delta$. Os autovalores do operador $-\Delta$ associado a $\mathrm{v}_{l k}$ são da forma $\lambda_{l k}=l^{2}+k^{2}$. A aproximação em série dos campos da velocidade e o geopotencial em termos das funções base são dadas pela seguinte expressão:

$$
\left[\begin{array}{l}
u(x, y, t) \\
v(x, y, t) \\
z(x, y, t)
\end{array}\right]=\sum_{l=-\infty}^{\infty} \sum_{k=-\infty}^{\infty}\left[\begin{array}{c}
\hat{u}_{l k}(t) \\
\hat{v}_{l k}(t) \\
\hat{z}_{l k}(t)
\end{array}\right] e^{i(l x+k y)}
$$

onde, $\hat{u}_{l k}(t), \hat{u}_{l k}(t)$ e $\hat{z}_{l k}(t)$ são os chamados coeficientes espectrais, e devem satisfazer as condições $\hat{u}_{l k}(t)=\hat{u}_{-l,-k}^{*}(t), \hat{v}_{l k}(t)=\hat{v}_{-l,-k}^{*}(t)$ e $\hat{z}_{l k}(t)=\hat{z}_{-l,-k}^{*}(t)$ para que $u, v$ e $z$ sejam reais ( $*$ denota o complexo conjugado).

(2.24) é uma aproximação com infinitos termos, e a discretização é baseada no truncamento da série para um número finito das funções base. Para isto definimos

$$
I_{N}=\left\{(l, k):-\frac{N}{2} \leq l, k \leq \frac{N}{2}\right\},
$$

como sendo o conjunto que define o nível de truncamento, enquanto que

$$
U_{N}=\operatorname{span}\left\{\mathrm{v}_{l k}=e^{i l x+i k y}:(l, k) \in I_{N}\right\}
$$

define as funções base a serem usadas para aproximar os campos da velocidade e geopotencial com base no truncamento definido por $I_{N}$. Também definimos o operador projeção $P_{N}: H_{p e r} \rightarrow U_{N}$ que leva uma função periódica do tipo (2.24) a uma função em $U_{N}$ do tipo:

$$
\left[\begin{array}{c}
u_{N}(x, y, t) \\
v_{N}(x, y, t) \\
z_{N}(x, y, t)
\end{array}\right]=\sum_{l, k \in I_{N}}\left[\begin{array}{c}
\hat{u}_{l k}(t) \\
\hat{v}_{l k}(t) \\
\hat{z}_{l k}(t)
\end{array}\right] e^{i(l x+k y)}
$$


O método de Galerkin Linear consiste na aplicação do operador $P_{N}$ ao sistema (2.19)(2.21), obtendo:

$$
\begin{aligned}
u_{N}^{n+1}-\Delta t v_{N}^{n+1}+\Delta t z_{N, x}^{n+1}-\nu_{0} \Delta t \Delta u_{N}^{n+1} & =P_{N}\left(r_{1 a}^{n-1}+r_{1 b}^{n}\right) \\
v_{N}^{n+1}+\Delta t u_{N}^{n+1}+\Delta t z_{N, y}^{n+1}-\nu_{0} \Delta t \Delta v_{N}^{n+1} & =P_{N}\left(r_{2 a}^{n-1}+r_{2 b}^{n}\right) \\
z_{N}^{n+1}+g_{0} \Delta t\left(u_{N, x}^{n+1}+v_{N, y}^{n+1}\right)-\kappa_{0} \Delta t \Delta z_{N}^{n+1} & =P_{N}\left(r_{3 a}^{n-1}+r_{3 b}^{n}\right)
\end{aligned}
$$

Substituindo (2.27) no sistema anterior e tendo em conta a ortogonalidade das funções base o seguinte sistema é obtido nos coeficientes espectrais para $(l, k) \in I_{N}$ :

$$
\left[\begin{array}{ccc}
\left(1+\nu_{0} \Delta t \lambda_{l k}\right) & -\Delta t & \mathrm{i} l \Delta t \\
\Delta t & \left(1+\nu_{0} \Delta t \lambda_{l k}\right) & \mathrm{i} k \Delta t \\
\mathrm{i} l g_{0} \Delta t & \mathrm{i} k g_{0} \Delta t & \left(1+\kappa_{0} \Delta t \lambda_{l k}\right)
\end{array}\right] \cdot\left[\begin{array}{c}
\hat{u}_{l k}^{n+1} \\
\hat{v}_{l k}^{n+1} \\
\hat{z}_{l k}^{n+1}
\end{array}\right]=\left[\begin{array}{c}
\hat{r}_{1 a, l k}^{n-1}+\hat{r}_{1 b, l k}^{n} \\
\hat{r}_{2 a, l k}^{n-1}+\hat{r}_{2 b, l k}^{n} \\
\hat{r}_{3 a, l k}^{n-1}+\hat{r}_{3 b, l k}^{n}
\end{array}\right]
$$

Para desacoplar o sistema forma-se o sistema nas variáveis $\hat{u}_{l k}^{n+1}$ e $\hat{v}_{l k}^{n+1}$ obtido das duas primeiras equações de $(2.31)$ :

$$
\left[\begin{array}{cc}
\left(1+\nu_{0} \Delta t \lambda_{l k}\right) & -\Delta t \\
\Delta t & \left(1+\nu_{0} \Delta t \lambda_{l k}\right)
\end{array}\right] \cdot\left[\begin{array}{c}
\hat{u}_{l k}^{n+1} \\
\hat{v}_{l k}^{n+1}
\end{array}\right]=\left[\begin{array}{c}
\hat{r}_{1 a, l k}^{n-1}+\hat{r}_{1 b, l k}^{n}-\mathrm{i} l \Delta t \hat{z}_{l k}^{n+1} \\
\hat{r}_{2 a, l k}^{n-1}+\hat{r}_{2 b, l k}^{n}-\mathrm{i} k \Delta t \hat{z}_{l k}^{n+1}
\end{array}\right]
$$

cuja solução é:

$$
\left[\begin{array}{c}
\hat{u}_{l k}^{n+1} \\
\hat{v}_{l k}^{n+1}
\end{array}\right]=\frac{1}{\theta_{l k}}\left[\begin{array}{c}
\hat{s}_{1, l k}-\mathrm{i}\left(l \Delta t\left(1+\nu_{0} \Delta t \lambda_{l k}\right)+k \Delta^{2} t\right) \hat{z}_{l k}^{n+1} \\
\hat{s}_{2, l k}+\mathrm{i}\left(l \Delta^{2} t-k \Delta t\left(1+\nu_{0} \Delta t \lambda_{l k}\right)\right) \hat{z}_{l k}^{n+1}
\end{array}\right]
$$

onde:

$$
\begin{aligned}
\mathrm{i} & =\sqrt{-1} \\
\theta_{l k} & =\left(1+\nu_{0} \Delta t \lambda_{l k}\right)^{2}+\Delta^{2} t \\
\hat{s}_{1, l k} & =\left(1+\nu_{0} \Delta t \lambda_{l k}\right)\left(\hat{r}_{1 a, l k}^{n-1}+\hat{r}_{1 b, l k}^{n}\right)+\Delta t\left(\hat{r}_{2 a, l k}^{n-1}+\hat{r}_{2 b, l k}^{n}\right) \\
\hat{s}_{2, l k} & =-\Delta t\left(\hat{r}_{1 a, l k}^{n-1}+\hat{r}_{1 b, l k}^{n}\right)+\left(1+\nu_{0} \Delta t \lambda_{l k}\right)\left(\hat{r}_{2 a, l k}^{n-1}+\hat{r}_{2 b, l k}^{n}\right)
\end{aligned}
$$


Multiplicando a primeira equação de (2.32) por i $l$ e a segunda por i $k$ ( o que equivale a construir o divergente no espaço físico) obtém-se:

$$
\mathrm{i}\left(l \hat{u}_{l k}^{n+1}+k \hat{v}_{l k}^{n+1}\right)=\frac{1}{\theta_{l k}}\left(\mathbf{i}\left(l \hat{s}_{1, l k}+k \hat{s}_{2, l k}\right)+\Delta t\left(1+\nu_{0} \Delta t \lambda_{l k}\right) \lambda_{l k} \hat{z}_{l k}^{n+1}\right)
$$

A terceira equação de (2.31) pode-se expressar como:

$$
\hat{z}_{l k}^{n+1}=\frac{1}{1+\kappa_{0} \Delta t \lambda_{l k}}\left(\hat{r}_{3 a, l k}^{n-1}+\hat{r}_{3 b, l k}^{n}-\mathbf{i} g_{0} \Delta t\left(l \hat{u}_{l k}^{n+1}+k \hat{v}_{l k}^{n+1}\right)\right)
$$

A substituição de (2.34) em (2.35) produz então a solução $\hat{z}_{l k}^{n+1}$ :

$$
\hat{z}_{l k}^{n+1}=\frac{1}{\alpha_{l k}}\left(\hat{r}_{3 a, l k}^{n-1}+\hat{r}_{3 b, l k}^{n}-\mathrm{i} \frac{g_{0} \Delta t}{\theta_{l k}}\left(l s_{1, l k}+k s_{2, l k}\right)\right)
$$

onde, $\theta_{l k}, s_{1, l k}$ e $s_{2, l k}$ são definidas em (2.33) e $\alpha_{l k}$ é dada pela relação:

$$
\alpha_{l k}=1+\frac{g_{0} \Delta^{2} t \lambda_{l k}}{\theta_{l k}}\left(1+\nu_{0} \Delta t \lambda_{l k}\right)+\kappa_{0} \Delta t \lambda_{l k}
$$

Concluindo, a solução do sistema (2.31) é obtida avaliando primeiro $\hat{z}_{l k}^{n+1}$ de (2.36), seguindo o cálculo de $\hat{u}_{l k}^{n+1}$ e $\hat{u}_{l k}^{n+1}$ de (2.32) (com $\hat{z}_{l k}^{n+1}$ já conhecida).

Todos os termos em (2.32) e (2.36) podem ser avaliados exatamente, exceto os termos não lineares em (2.23). A implementação destes tem que ser feita de maneira eficiente pois disto depende a eficiência total do método. Além disso, é necessário calcular os termos não lineares corretamente, evitando o 'aliasing' que poderia gerar instabilidade devido a transferência espuria de energia de modos altos para modos baixos.

Para a inversão de (2.27) os campos são avaliados nos pontos da malha:

$$
\mathbb{J}_{N}=\left\{\left(x_{r}, y_{s}\right): \quad x_{r}=\frac{2 \pi(r-1)}{N}, y_{r}=\frac{2 \pi(s-1)}{N}, \quad r, s=1, \cdots, N\right\} .
$$

Multiplicando por $e^{-i\left(\tilde{l}_{r}+\tilde{k} y_{s}\right)}$ e efetuando a soma para $r, s=1, \cdots, N$ obtemos:

$$
\sum_{r=1}^{N} \sum_{s=1}^{N}\left[\begin{array}{c}
u_{N}\left(x_{r}, y_{s}, t\right) \\
v_{N}\left(x_{r}, y_{s}, t\right) \\
z_{N}\left(x_{r}, y_{s}, t\right)
\end{array}\right] e^{-i\left(\tilde{l} x_{r}+\tilde{k} y_{s}\right)}=\sum_{l, k \in I_{N}}\left[\begin{array}{c}
\hat{u}_{l k}(t) \\
\hat{v}_{l k}(t) \\
\hat{z}_{l k}(t)
\end{array}\right] \sum_{r=1}^{N} \sum_{s=1}^{N} e^{i\left((l-\tilde{l}) x_{r}+(k-\tilde{k}) y_{s}\right)}
$$


e tendo em conta a identidade,

$$
\sum_{r=1}^{N} \sum_{s=1}^{N} e^{i\left((l-\tilde{l}) x_{r}+(k-\tilde{k}) y_{s}\right)}=\left\{\begin{array}{cccc}
0, & l \neq \tilde{l} & \text { ou } & k \neq \tilde{k} \\
N^{2}, & l=\tilde{l} & \mathrm{e} & k=\tilde{k}
\end{array},\right.
$$

a seguinte relação para os coeficientes espectrais em função dos valores na malha é obtida:

$$
\left[\begin{array}{c}
\hat{u}_{l k}(t) \\
\hat{v}_{l k}(t) \\
\hat{z}_{l k}(t)
\end{array}\right]=\frac{1}{N^{2}} \sum_{x_{r}, y_{s} \in J_{N}}\left[\begin{array}{c}
u_{N}\left(x_{r}, y_{s}, t\right) \\
v_{N}\left(x_{r}, y_{s}, t\right) \\
z_{N}\left(x_{r}, y_{s}, t\right)
\end{array}\right] e^{-i\left(l x_{r}+k y_{s}\right)}
$$

Sendo os coeficientes espectrais complexos seria necessária uma matriz real de dimensão $2 N \times 2 N$ para armazenar todos eles, mas sendo os campos reais tém-se propriedades de simetria (como por exemplo $\hat{u}_{l k}=\hat{u}_{-l,-k}^{*}, \quad \hat{u}_{l, 0}=\hat{u}_{-l, 0}^{*}$ ) que permitem reduzir a alocação para uma matriz de tamanho $N \times N$, equivalente à dimensão da matriz necessária para armazenar os campos na malha $\mathbb{J}_{n}$. Estas propriedades são utilizadas para construir uma Transformada Rápida de Fourier em duas dimensões (FFT-2D) a partir da Transformada Rápida de Fourier em uma dimensão (FFT-1D): aplica-se $N$ FFTs-1D numa direção seguidas de $N$ FFTs-1D na outra direção ( em (2.39) se soma primeiro em $s$ e $\operatorname{logo}$ em $r$ ). Sendo o custo computacional para avaliar uma FFT-1D da ordem $\vartheta\left(N \log _{2} N\right)$, o custo computacional para avaliar uma FFT-2D é da ordem $\vartheta\left(N^{2} \log _{2}\left(N^{2}\right)\right) \quad\left(\sim \vartheta\left(N\left(N \log _{2} N\right)\right)+\vartheta\left(N\left(N \log _{2} N\right)\right)\right)$, o que constitui um modo eficiente de avaliar todos os termos em (2.27) ou em (2.38) se comparado com a avaliação direta, a qual tem um custo computacional da ordem de $\vartheta\left(N^{4}\right)$ operações. Na literatura a avaliação de (2.39) por FFT-2D é chamada de Transformada Direta e a avaliação de (2.27) é chamada de Transformada Inversa. Estas transformadas são eficientes computacionalmente quando $N$ é fatorável em primos pequenos (geralmente 2, 3, 5 e 7).

Para avaliar os termos não lineares usamos o método das Transformadas, gerando os valores das funções envolvidas nos pontos da malha (espaço físico), construindo os produtos dos termos nos pontos da malha e transformando de volta ao espaço espectral (coeficientes espectrais) usando FFT-2D. Como explicado para o caso de uma dimensão o produto de duas funções em $U_{N}$ não pertence mais a $U_{N}$ (na realidade pertence a $U_{2 N}$ ). Na malha $\mathbb{J}_{N} U_{N}$ os modos $\mathrm{v}_{l+\frac{N}{2}, k+\frac{N}{2}},(l, k) \in I_{N}$ coincidem com os modos $\overline{\mathrm{v}}_{\frac{N}{2}-l, \frac{N}{2}-k},(l, k) \in I_{N}$. 
Esta retroalimentação da energia nos modos em $U_{N}$, conhecida como 'aliasing' é evitada avaliando os valores no espaço físico numa malha maior, tal como foi feito numa dimensão $\left(\mathbb{J}_{3 N / 2}\right.$ ao invés de $\left.\mathbb{J}_{N}\right)$.

A seguir são mostrados os detalhes para a implementação de $\left(\widehat{u^{2}}\right)_{l k}, \quad(l, k) \in I_{N}$ :

a1) Conhecidos os coeficientes espectrais dos campos para $(l, k) \in I_{N}$, definimos:

$$
\tilde{u}_{l k}=\left\{\begin{array}{c}
\hat{u}_{l, k}, \quad \text { se } \quad(l, k) \in I_{N} \\
0, \quad \text { se } \quad(l, k) \in I_{\frac{3}{2} N}-I_{N}
\end{array}\right.
$$

a2) Avaliar os valores de $u\left(x_{r}, y_{s}\right)$ para $x_{r}, y_{s} \in J_{3 N / 2}$ por meio de uma FFT-2D inversa de tamanho $\frac{3}{2} N \times \frac{3}{2} N$.

a3) Avaliar $u^{2}\left(x_{r}, y_{s}\right)$ na malha $J_{\frac{3}{2} N}$.

a4) Conhecidos os valores de $u^{2}\left(x_{r}, y_{s}\right)$ para $x_{r}, y_{s} \in J_{3 / 2 N}$ avaliar os coeficientes espectrais usando uma FFT-2D de tamanho $\frac{3}{2} N \times \frac{3}{2} N$. A chave é observar que com esta escolha os coeficientes espectrais do produto $\left(\widehat{u^{2}}\right)_{l k}$ para $(l, k) \in I_{N}$ ficam livres de aliasing. Com efeito, $\widehat{u^{2}}$ pertence a $U_{2 N}$, porem os coeficientes dos modos $\mathrm{v}_{l, k}$ com $(l, k) \in\left(I_{2 N}-I_{\frac{3}{2} N}\right)$ devem ser absorvidos pêlos coeficientes espectrais de $\mathrm{v}_{l, k}$ com $(l, k) \in\left(I_{\frac{3}{2} N}-I_{N}\right)$, pelo qual os coeficientes espectrais de $\mathrm{v}_{l, k} \operatorname{com}(l, k) \in I_{N}$ ficam livres de aliasing, o que é nosso objetivo.

Os coeficientes espectrais dos outros termos não lineares em (2.23) são avaliados da mesma forma. Enquanto avaliamos os coeficientes espectrais dos termos lineares em (2.22) por:

$$
\begin{aligned}
& \hat{r}_{1 a, l k}^{n-1}=\left(1-\nu_{0} \Delta t \lambda_{l k}\right) \hat{u}_{l k}^{n-1}+\Delta t \hat{v}_{l k}^{n-1}-\mathrm{i} l \Delta t \hat{z}_{l k}^{n-1} \\
& \hat{r}_{2 a, l k}^{n-1}=-\Delta t \hat{u}_{l k}^{n-1}+\left(1-\nu_{0} \Delta t \lambda_{l k}\right) \hat{v}_{l k}^{n-1}-\mathrm{i} k \Delta t \hat{z}_{l k}^{n-1} \\
& \hat{r}_{3 a, l k}^{n-1}=-\mathbf{i} \Delta t g_{0}\left(l \hat{u}_{l k}^{n-1}+k \hat{v}_{l k}^{n-1}\right)+\left(1-\nu_{0} \Delta t \lambda_{l k}\right) \hat{z}_{l k}^{n-1}
\end{aligned}
$$

\subsubsection{Implementação numérica}

Com base na discussão anterior, descrevemos a implementação do sistema (2.28)-(2.30) e analisamos o custo computacional deste método:

b1) Assuma-se conhecido no espaço espectral $\hat{u}_{l k}^{n-1}, \hat{v}_{l k}^{n-1}, \hat{z}_{l k}^{n-1}$ para $(l, k) \in I_{N}$ (ver 
(2.25)), e no espaço físico assuma-se conhecido $u^{n}, u_{x}^{n}, u_{y}^{n}, v^{n}, v_{x}^{n}, v_{y}^{n}, z^{n}, z_{x}^{n}, z_{y}^{n}$ na malha $J_{\frac{3}{2} N}(\operatorname{ver}(2.38))$

b2) Avaliar na malha $J_{\frac{3}{2} N}$ os produtos:

$r_{1 b}^{n}=u^{n} u_{x}^{n}+v^{n} u_{y}^{n}, \quad r_{2 b}^{n}=u^{n} v_{x}^{n}+v^{n} v_{y}^{n}$, e $r_{3 b}^{n}=\left(z^{n} u^{n}\right)_{x}+\left(z^{n} v^{n}\right)_{y}+F$.

b3) Usar FFT-2D para avaliar os coeficientes espectrais das três expressões em b2):

$\hat{r}_{1 b, l k}^{n} \hat{r}_{1 b, l k}^{n}$ e $\hat{r}_{1 b, l k}^{n}$ (os coeficientes espectrais para $(l, k) \in I_{N}$ estão livres de aliasing (ver a4)). Nesta avaliação dos coeficientes espectrais são usadas 3 FFT-2D de tamanho $\frac{3}{2} N \times \frac{3}{2} N$.

b4) Avaliar $\hat{r}_{1 a, l k}^{n-1}, \hat{r}_{2 a, l k}^{n-1}$ e $\hat{r}_{3 a, l k}^{n-1}$, em (2.40).

b5) Obter $\hat{z}_{l k}^{n+1}$ de (2.36) e a seguir obter $\hat{u}_{l k}^{n+1}$ e $\hat{v}_{l k}^{n+1}$ de $(2.32)$ para $(l, k) \in I_{N}$.

Os próximos passos se seguem para atualizar os valores para o avanço no tempo.

b6) Atualizar no espaço espectral: $\left(\hat{u}_{l k}^{n-1} \leftarrow \hat{u}_{l k}^{n}, \hat{v}_{l k}^{n-1} \leftarrow \hat{v}_{l k}^{n}, \hat{z}_{l k}^{n-1} \leftarrow \hat{z}_{l k}^{n}\right) \mathrm{e}$ $\left(\hat{u}_{l k}^{n} \leftarrow \hat{u}_{l k}^{n+1}, \hat{v}_{l k}^{n} \leftarrow \hat{v}_{l k}^{n+1}, \hat{z}_{l k}^{n} \leftarrow \hat{z}_{l k}^{n+1}\right)$ para $(l, k) \in I_{N}$.

b7) Usando a1 e a2 avaliar $u^{n}, u_{x}^{n}, u_{y}^{n}, v^{n}, v_{x}^{n}, v_{y}^{n}, z^{n}, z_{x}^{n}$ e $z_{y}^{n}$ na malha $J_{\frac{3}{2} N}$. Nesta avaliação das funções no espaço físico são usadas 9 FFT-2D de tamanho $\frac{3}{2} N \times \frac{3}{2} N$.

b8) Voltar ao passo (b2).

De b3 e b7 temos que em cada iteração no tempo são avaliadas 12 FFT-2D de tamanho $\frac{3}{2} N \times \frac{3}{2} N$. Tendo em conta que as FFT's dominam o custo computacional, estima-se um custo por iteração no tempo da ordem de $\vartheta\left(27 N^{2} \log _{2}\left(\frac{3 N}{2}\right)^{2}\right)$.

Como utilizamos um esquema de 3 níveis no tempo (que possui modos computacionais com um período $2 \Delta t$ ), aplica-se a seguinte filtragem (suave) no tempo (ver [30]):

$\hat{u}^{n}=\hat{u}^{n}+\gamma\left(\hat{u}^{n+1}-2 \hat{u}^{n}+\hat{u}^{n-1}\right)$

$\hat{v}^{n}=\hat{v}^{n}+\gamma\left(\hat{v}^{n+1}-2 \hat{v}^{n}+\hat{v}^{n-1}\right)$

$\hat{z}^{n}=\hat{z}^{n}+\gamma\left(\hat{z}^{n+1}-2 \hat{z}^{n}+\hat{z}^{n-1}\right)$

onde escolhemos $\gamma=0.025$. 


\subsubsection{Resultados numéricos}

A seguir apresentamos resultados da simulação numérica das equações de água rasa pelo método de Galerkin linear. O seguinte sub-sistema diagnóstico de (2.16)-(2.18) é obtido ao manter só a coordenada latitudinal $y$ e deixando fora os termos não lineares:

$$
\begin{aligned}
-v & =\nu_{0} \frac{\partial^{2} u}{\partial y^{2}} \\
u+\frac{\partial z}{\partial y} & =\nu_{0} \frac{\partial^{2} v}{\partial y^{2}} \\
g_{0} \frac{\partial v}{\partial y} & =\nu_{0} \frac{\partial^{2} z}{\partial y^{2}}+F(y)
\end{aligned}
$$

No sistema (2.41) temos equilíbrio da circulação secundária $v$ e a difusão do fluxo zonal $u$ na primeira equação; equilíbrio geostrófico do vento zonal $u$ para $\nu_{0}$ pequeno na segunda equação e o equilíbrio entre o termo que força o fluido e a conveção meridional, a qual força o fluxo zonal do geopotencial na terceira equação. Deixando só os termos dominantes no balanço anterior, a equação (2.41) se reduz a:

$$
\begin{aligned}
-v & =\nu_{0} \frac{\partial^{2} u}{\partial y^{2}} \\
u+\frac{\partial z}{\partial y} & =0 \\
g_{0} \frac{\partial v}{\partial y} & =F(y)
\end{aligned}
$$

Tomando $F=\frac{1}{100} \cos (y)$, as funções seguintes:

$$
\begin{aligned}
u & =\frac{1}{100 \nu_{0} g_{0}} \operatorname{sen}(y) \\
v & =\frac{1}{100 g_{0}} \operatorname{sen}(y) \\
z & =\frac{1}{100 \nu_{0} g_{0}} \cos (y)
\end{aligned}
$$

constituem uma solução exata do sistema (2.42).

A solução (2.43) do sistema (2.42) é usada como condição inicial para a implementação das equações de água rasa (2.28)-(2.30), e o sistema é perturbado através da forçante $F=\frac{L^{2}}{g f^{3}} \cos (x) e^{-x y / 4}$. 


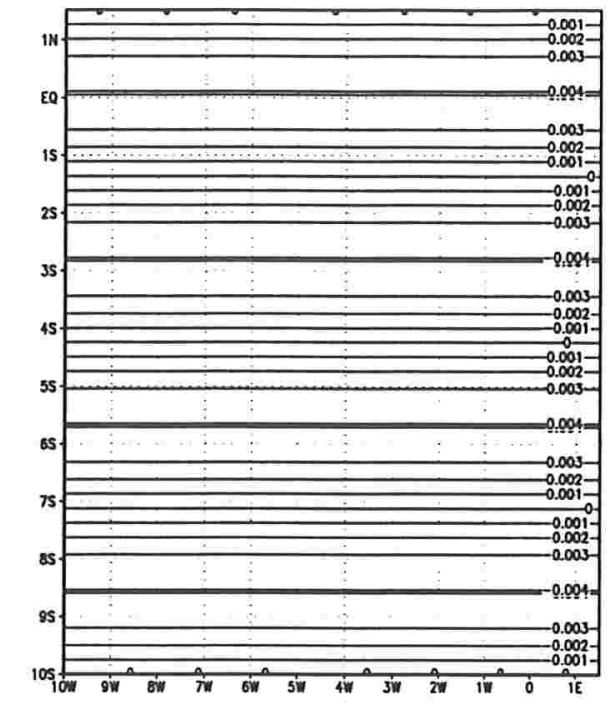

(a)

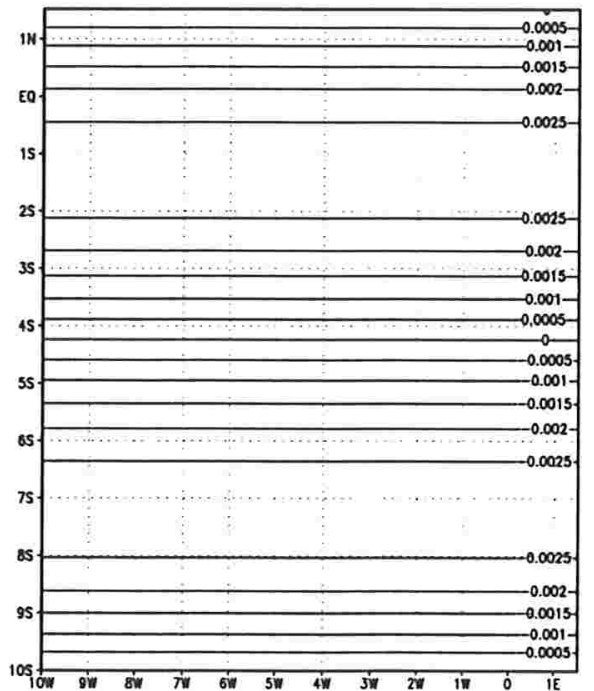

(c)

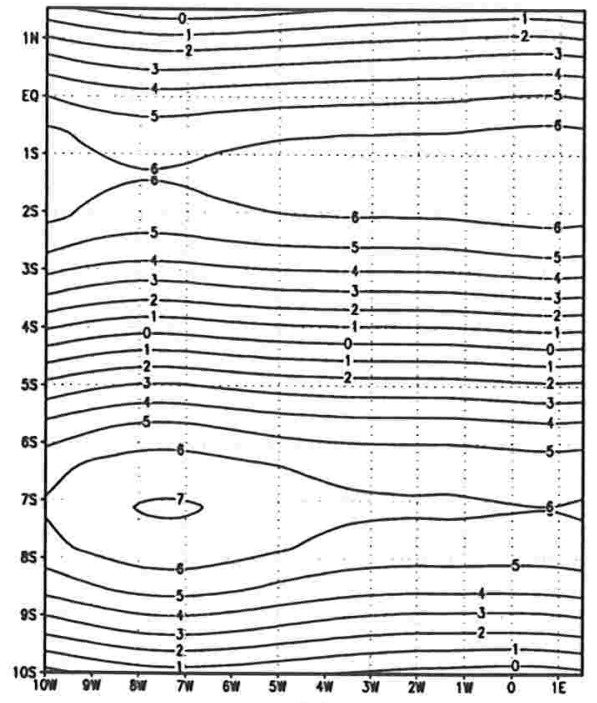

(b)

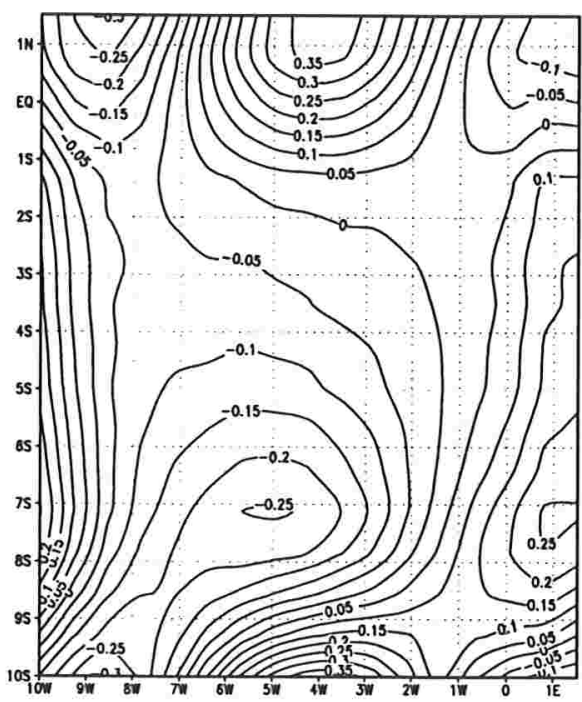

(d)

Figura 2.16: Eq. de água rasa: campos da velocidade

a) $\mathrm{u}: t=9 \min$. b) u: $t=90 \min$. c) $\mathrm{v}: t=9 \min$. d) $\mathrm{v}: t=90 \min$. 

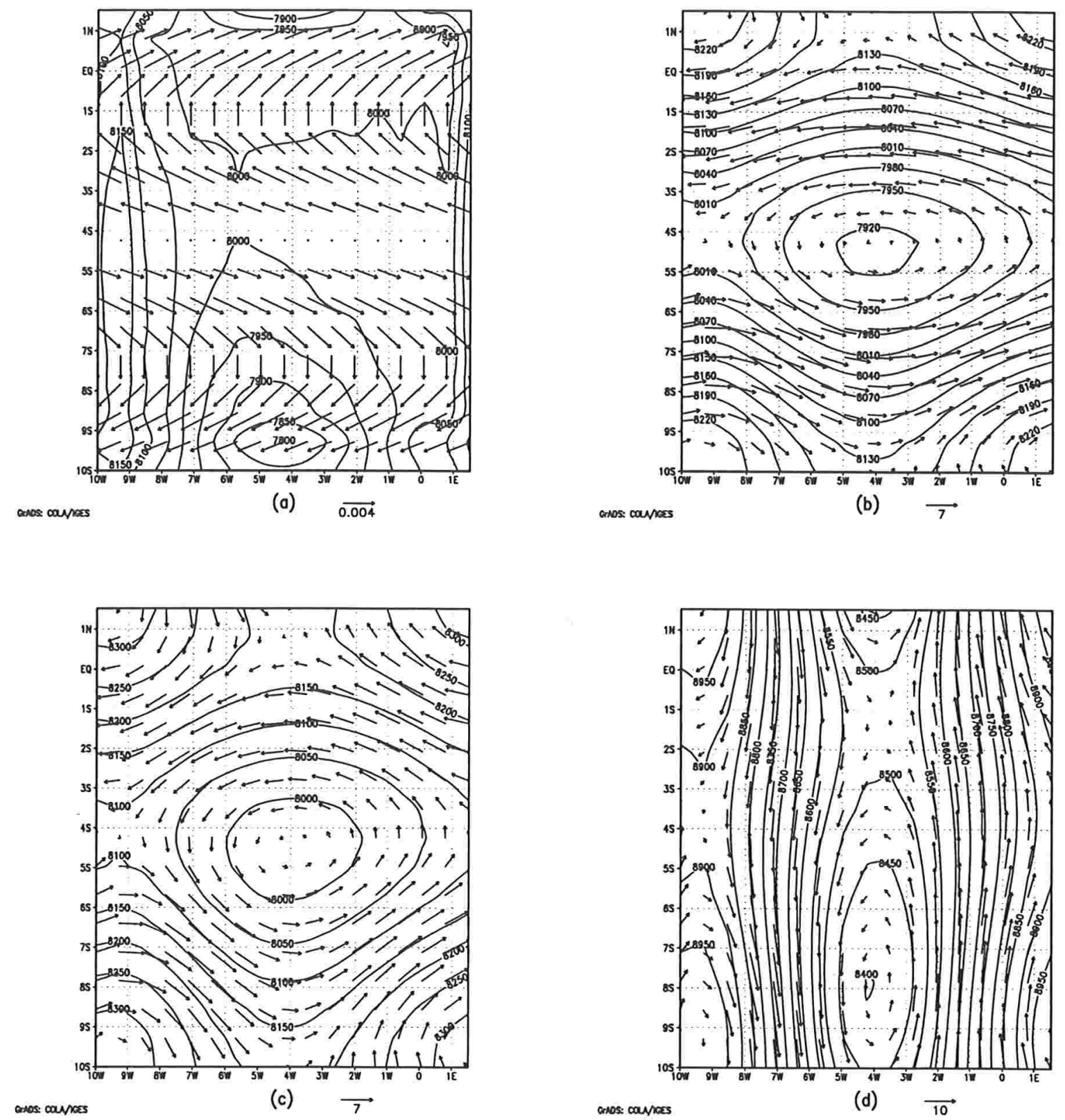

Figura 2.17: Eq. de água rasa: Linhas de corrente e geopotencial $(z+h)$

a) $t=9$ min. b) $t=15 \mathrm{~h}$. c) $t=30 \mathrm{~h}$. d) $t=150 \mathrm{~h}$. 


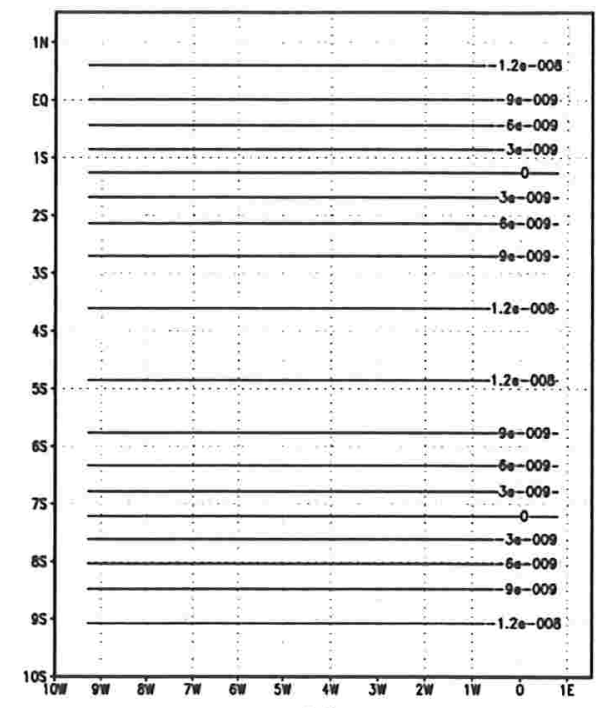

(a)

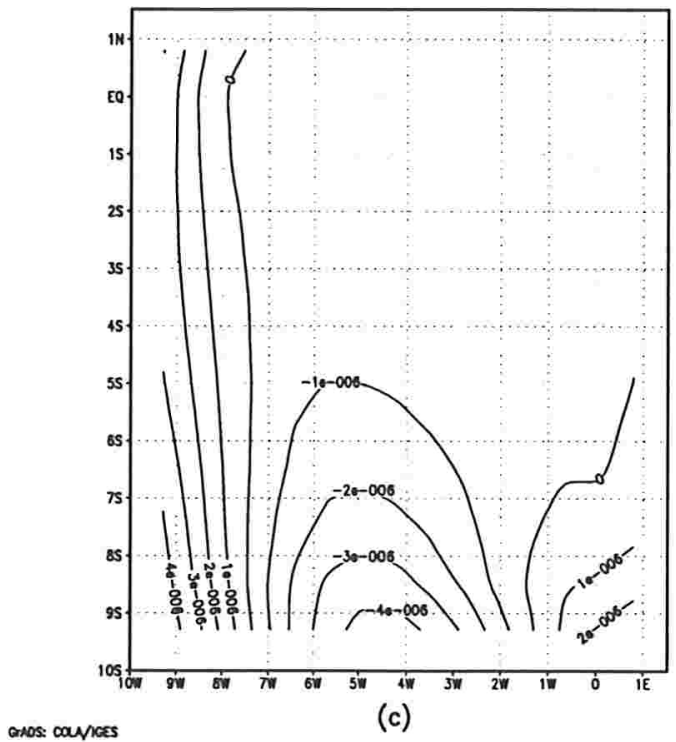

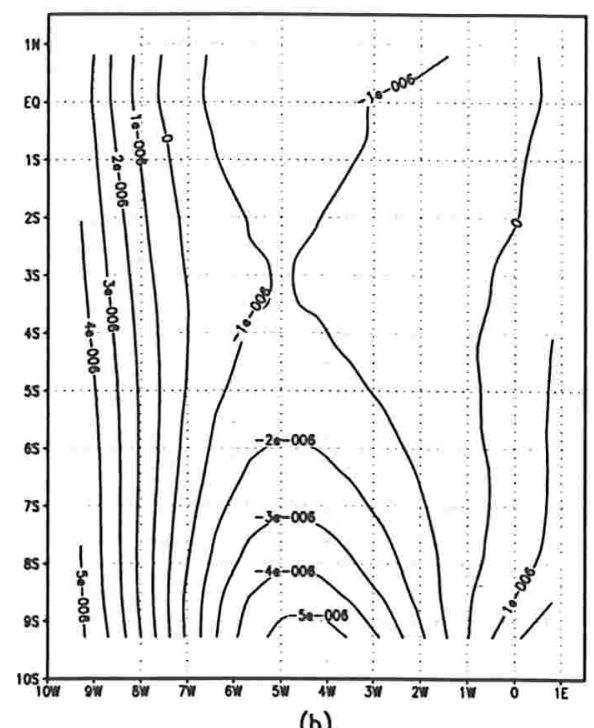

aros can wors

(b)

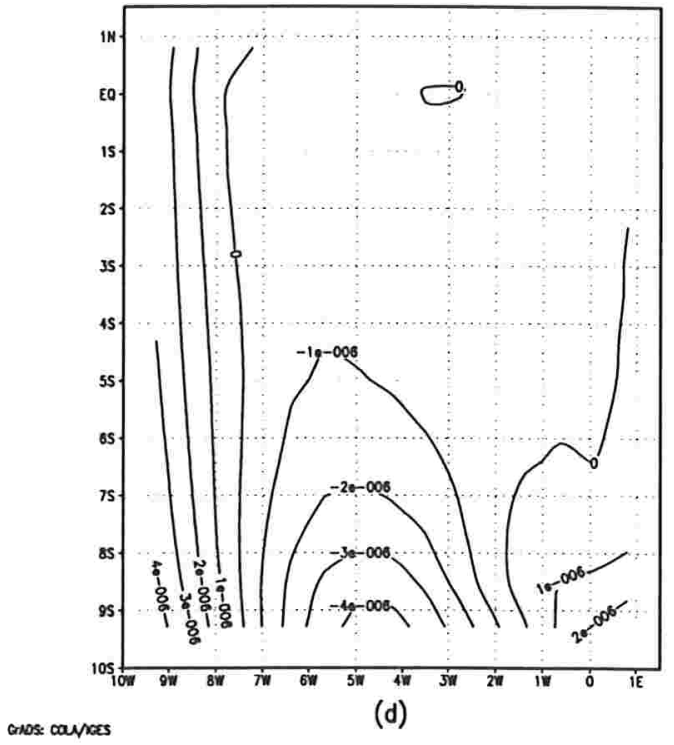

Figura 2.18: Eq. de água rasa: divergência
a) $t=9 \mathrm{~min}$.
b) $t=15 \mathrm{~h}$.
(c) $t=30 \mathrm{~h}$.
(d) $t=150 \mathrm{~h}$. 
Nas próximas simulações usamos os parâmetros: $L=1080 \mathrm{~km}, \mathrm{f}^{-1}=3$ horas, $h=8$ $\mathrm{km}, g=10 \mathrm{~ms}^{-1}, \nu=\kappa=2.25 \times 10^{6} \mathrm{~ms}^{-2}$. Com esta escolha temos os valores para as equações adimensionalizadas: $g_{0}=8$, e $\nu_{0}=\kappa_{0}=\frac{1}{48}$.

Os campos iniciais da velocidade em $t_{0}=0$ são constantes $u_{0}=v_{0}=0$ e $z_{0}+h=7800 \mathrm{~km}$, na figura (2.16) (a) e (c) são mostradas os campos da velocidade em $t_{1}=9$ minutos obtida usando um esquema explícito, enquanto os campos da velocidade logo de 90 minutos de simulação ( $\Delta t=9$ minutos) são mostradas na figura (2.16) (b) e (d).

A evolução do geopotencial e das linhas de corrente é mostrada na figura (2.17) nos instantes $t=0,15,30$ e 150 horas. Pode observar-se a formação de um centro de baixa pressão ao-redor do qual as linhas de corrente circulam em sentido antihorário e a formação de um centro de alta pressão com circulação anti-horária (esta direção das linhas de corrente concordam com o fato do parâmetro de Coriolis ser positivo.

Na figura (2.18) se mostra o divergente (em m/seg ) para os mesmos valores dos parâmetros e tempos anteriores. Observa-se um comportamento quase não-divergente do campo de velocidades ainda que este campo não seja pequeno. 


\subsubsection{Descrição do método de Galerkin Não Linear}

Começamos a descrição do método de Galerkin não linear com a decomposição do espaço das auto-funções $\mathbb{U}_{N}$ (definido em $(2.27)$ ) em dois conjuntos: $\mathbb{U}_{N / 2}$ (modos baixos) e $\mathbb{W}_{N}=\mathbb{U}_{N}-\mathbb{U}_{N / 2}$ (modos altos). Observando que a projeção $P_{N}$ verifica: $P_{N}=P_{N / 2}+Q_{N}$ $\left(P_{N / 2}: \mathbb{H}_{p e r} \rightarrow \mathbb{U}_{N / 2}\right.$ e $\left.Q_{N / 2}: \mathbb{H}_{p e r} \rightarrow \mathbb{W}_{N}\right)$ os campos da velocidade e geopotencial são decompostos em duas componentes: uma contendo só modos baixos (em $\mathbb{U}_{N / 2}$ ) e a outra componente contendo só modos altos $\left(\mathrm{em} \mathbb{W}_{N}\right)$,

$$
\begin{array}{lll}
u_{N}=p_{1}+q_{1}, & \left(p_{1}=P_{N / 2}(u),\right. & \left.q_{1}=Q_{N}(u)\right) \\
v_{N}=p_{2}+q_{2}, & \left(p_{2}=P_{N / 2}(v),\right. & \left.q_{2}=Q_{N}(v)\right) \\
z_{N}=p_{3}+q_{3}, & \left(p_{3}=P_{N / 2}(z),\right. & \left.q_{3}=Q_{N}(z)\right) \\
F_{N}=F_{1}+F_{2}, & \left(F_{1}=P_{N / 2} F,\right. & \left.F_{2}=Q_{N} F\right)
\end{array}
$$

Aplicando as projeções $P_{N / 2}$ e $Q_{N}$ às equações de água rasa (2.16)-(2.18) obtém-se o sistema:

$$
\begin{aligned}
\frac{\partial p_{1}}{\partial t}-p_{2}+\frac{\partial p_{3}}{\partial x}-\nu_{0} \Delta p_{1} & =s_{1} \\
\frac{\partial p_{2}}{\partial t}+p_{1}+\frac{\partial p_{3}}{\partial y}-\nu_{0} \Delta p_{2} & =s_{2} \\
\frac{\partial p_{3}}{\partial t}+g_{0}\left(\frac{\partial p_{1}}{\partial x}+\frac{\partial p_{2}}{\partial x}\right)-\kappa_{0} \Delta p_{3} & =s_{3} \\
\frac{\partial q_{1}}{\partial t}-q_{2}+\frac{\partial q_{3}}{\partial x}-\nu_{0} \Delta q_{1} & =s_{4} \\
\frac{\partial q_{2}}{\partial t}+q_{1}+\frac{\partial q_{3}}{\partial y}-\nu_{0} \Delta q_{2} & =s_{5} \\
\frac{\partial q_{3}}{\partial t}+g_{0}\left(\frac{\partial q_{1}}{\partial x}+\frac{\partial q_{2}}{\partial x}\right)-\kappa_{0} \Delta q_{3} & =s_{6}
\end{aligned}
$$

As equações (2.45)-(2.47) serão chamadas de equações dos modos baixos e (2.48)-(2.50) de equações dos modos altos. Os termos $s_{i},(i=1, \cdots, 6)$ são definidos por:

$$
\begin{aligned}
& s_{1}=P_{N / 2}\left(-\left(p_{1}+q_{1}\right)\left(p_{1}+q_{1}\right)_{x}-\left(p_{2}+q_{2}\right)\left(p_{1}+q_{1}\right)_{y}\right) \\
& s_{2}=P_{N / 2}\left(-\left(p_{1}+q_{1}\right)\left(p_{2}+q_{2}\right)_{x}-\left(p_{2}+q_{2}\right)\left(p_{2}+q_{2}\right)_{y}\right)
\end{aligned}
$$




$$
\begin{aligned}
& s_{3}=P_{N / 2}\left(F-\left[\left(p_{3}+q_{3}\right)\left(p_{1}+q_{1}\right)\right]_{x}-\left[\left(p_{3}+q_{3}\right)\left(p_{2}+q_{2}\right)\right]_{y}\right) \\
& s_{4}=Q_{N / 2}\left(-\left(p_{1}+q_{1}\right)\left(p_{1}+q_{1}\right)_{x}-\left(p_{2}+q_{2}\right)\left(p_{1}+q_{1}\right)_{y}\right) \\
& s_{5}=Q_{N / 2}\left(-\left(p_{1}+q_{1}\right)\left(p_{2}+q_{2}\right)_{x}-\left(p_{2}+q_{2}\right)\left(p_{2}+q_{2}\right)_{y}\right) \\
& s_{6}=Q_{N / 2}\left(F-\left[\left(p_{3}+q_{3}\right)\left(p_{1}+q_{1}\right)\right]_{x}-\left[\left(p_{3}+q_{3}\right)\left(p_{2}+q_{2}\right)\right]_{y}\right)
\end{aligned}
$$

Nas equações dos modos baixos (2.45)-(2.47) desprezamos a interação dos modos altos nos termos não lineares. A justificativa é que estes modos contém menor quantidade de energia e sua contribuição no produto total é pequena quando $N$ é suficientemente grande (A energia cinética para $u_{N}$ é dada por: $E_{c}\left(u_{N}\right)=\frac{1}{2}\left(u_{N}, u_{N}\right)=\frac{1}{2} \sum_{i, j \in \mathbb{U}_{N}}\left|\hat{u}_{l k}\right|^{2} \mathrm{e}$ verifica $E_{c}\left(u_{N}\right)=E_{c}\left(p_{1}\right)+E_{c}\left(q_{1}\right)$. Sendo que os coeficientes espectrais satisfazem $\hat{u}_{l k} \rightarrow 0$ quando $(l, k) \rightarrow \infty$, temos que a maior parte da energia deve concentrar-se nos modos baixos a partir de um certo $N_{0}$ ). Com esta simplificação pode-se avaliar estes termos sem aliasing usando FFT-2D em malha menor que a usada no método de Galerkin linear. Logo (2.51)-(2.53) são aproximados por:

$$
\begin{aligned}
& s_{1} \simeq P_{N / 2}\left(-p_{1}\left(p_{1}+q_{1}\right)_{x}-q_{1} p_{1, x}-p_{2}\left(p_{1}+q_{1}\right)_{y}-q_{2} p_{1, y}\right) \\
& s_{2} \simeq P_{N / 2}\left(-p_{1}\left(p_{2}+q_{2}\right)_{x}-q_{1} p_{2, x}-p_{2}\left(p_{2}+q_{2}\right)_{y}-q_{2} p_{2, y}\right) \\
& s_{3} \simeq P_{N / 2}\left(F-\left(p_{3}\left(p_{1}+q_{1}\right)\right)_{x}-\left(q_{3} p_{1}\right)_{x}-\left(p_{3}\left(p_{2}+q_{2}\right)\right)_{y}-\left(q_{3} p_{2}\right)_{y}\right)
\end{aligned}
$$

enquanto que na equação dos modos altos (2.48)-(2.50), além de desprezar a interação dos modos altos, também desprezamos a interação entre modos altos e baixos. Resulta então que os modos altos são função dos modos baixos, permitindo que o sistema seja facilmente inversível. Com estas simplificações (2.54)-(2.55) são aproximadas por:

$$
\begin{aligned}
& s_{4} \simeq Q_{N}\left(-p_{1} p_{1, x}-p_{2} p_{1, y}\right) \\
& s_{5} \simeq Q_{N}\left(-p_{1} p_{2, x}-p_{2} p_{2, y}\right) \\
& s_{6} \simeq Q_{N}\left(F-\left(p_{3} p_{1}\right)_{x}-\left(p_{3} p_{2}\right)_{y}\right)
\end{aligned}
$$

Na seção 1.5 obtivemos estimativas de erro do método de Galerkin não linear (1.60) para as equações de água rasa (1.57)-(1.58) usando a variedade inercial aproximada (1.61) como 
um sistema diagnóstico descrevendo a evolução dos modos altos em função dos modos baixos. Com a suposição que as soluções das equações de água rasa são limitadas na norma $L_{2}$ provou-se que o método de Galerkin não linear deve satisfazer estimativas de erro da ordem de $\lambda_{N / 2}^{-3 / 2}$ (ver Teorema 1.2), enquanto que o método de Galerkin linear satisfaz estimativas da ordem $\lambda_{N}^{-1}$ (ver Teorema 1.3). Com base nestes resultados aproximamos as equações dos modos altos (2.48)-(2.50) por um sistema diagnóstico obtido ao eliminar a parte temporal dos modos altos nestas equações. Neste caso o método de Galerkin não linear é expresso pelo sistema:

$$
\begin{aligned}
\frac{\partial p_{1}}{\partial t}-p_{2}+\frac{\partial p_{3}}{\partial x}-\nu_{0} \Delta p_{1} & =s_{1} \\
\frac{\partial p_{2}}{\partial t}+p_{1}+\frac{\partial p_{3}}{\partial y}-\nu_{0} \Delta p_{2} & =s_{2} \\
\frac{\partial p_{3}}{\partial t}+g_{0}\left(\frac{\partial p_{1}}{\partial x}+\frac{\partial p_{2}}{\partial x}\right)-\kappa_{0} \Delta p_{3} & =s_{3} \\
-q_{2}+\frac{\partial q_{3}}{\partial x}-\nu_{0} \Delta q_{1} & =s_{4} \\
q_{1}+\frac{\partial q_{3}}{\partial y}-\nu_{0} \Delta q_{2} & =s_{5} \\
g_{0}\left(\frac{\partial q_{1}}{\partial x}+\frac{\partial q_{2}}{\partial x}\right)-\kappa_{0} \Delta q_{3} & =s_{6}
\end{aligned}
$$

onde $s_{i},(i=1, \cdots, 6)$ são também aproximados por (2.57)-(2.62).

Ao aplicar o método de Galerkin não linear para as equações de água rasa duas possibilidades de solução foram obtidas: o sistema (2.63)-(2.68) onde os modos altos são avaliados a partir do sistema diagnóstico (2.66)-(2.68), e o sistema (2.45)-(2.50) onde os modos altos são avaliados pelo sistema prognóstico (2.48)-(2.50).

A discretização no tempo para a parte temporal em (2.45)-(2.50) e (2.63)-(2.65) é baseada no esquema semi-implícito descrito na seção 2.1.1:

\begin{tabular}{|c|c|c|c|c|}
\hline Termo & $\frac{\partial \theta}{\partial t}$ & Linear (L) & Não linear (NL) & Forçante (F) \\
\hline Discretização & $\frac{\theta^{n+1}-\theta^{n-1}}{2 \Delta t}$ & $\frac{1}{2}\left(L^{n+1}+L^{n-1}\right)$ & $(N L)^{n}$ & $F$ \\
\hline
\end{tabular}


isto é, as derivadas temporais são aproximadas usando diferença central, uma media dos termos lineares nos tempos $t_{n+1}$ e $t_{n-1}$, os termos não lineares são avaliados no tempo $t_{n}$, e a forçante $F$ independe do tempo. O sistema diagnóstico (2.66)-(2.68) é resolvido para os modos altos assumindo-se conhecidos os modos baixos. A discretização temporal destes sistemas fica:

$$
\begin{aligned}
p_{1}^{n+1}-\Delta t p_{2}^{n+1}+\Delta t p_{3, x}^{n+1}-\nu_{0} \Delta t \Delta p_{1}^{n+1} & =S_{1}^{n-1}+2 \Delta t s_{1}^{n, n+1} \\
p_{2}^{n+1}+\Delta t p_{1}^{n+1}+\Delta t p_{3, y}^{n+1}-\nu_{0} \Delta t \Delta p_{2}^{n+1} & =S_{2}^{n-1}+2 \Delta t s_{2}^{n, n+1} \\
p_{3}^{n+1}+g_{0} \Delta t\left(p_{1 x}^{n+1}+p_{2 y}^{n+1}\right)-\kappa_{0} \Delta t \Delta p_{3}^{n+1} & =S_{3}^{n-1}+2 \Delta t s_{3}^{n, n+1} \\
\frac{\delta}{\Delta t} q_{1}^{n+1}-q_{2}^{n+1}+q_{3, x}^{n+1}-\nu_{0} \Delta q_{1}^{n+1} & =\frac{\delta}{\Delta t} S_{4}^{n-1}+\frac{2}{\eta} s_{4}^{n} \\
\frac{\delta}{\Delta t} q_{2}^{n+1}+q_{1}^{n+1}+q_{3, y}^{n+1}-\nu_{0} \Delta q_{2}^{n+1} & =\frac{\delta}{\Delta t} S_{5}^{n-1}+\frac{2}{\eta} s_{5}^{n} \\
\frac{\delta}{\Delta t} q_{3}^{n+1}+g_{0}\left(q_{1 x}^{n+1}+q_{2 y}^{n+1}\right)-\kappa_{0} \Delta q_{3}^{n+1} & =\frac{\delta}{\Delta t} S_{6}^{n-1}+\frac{2}{\eta} s_{6}^{n}
\end{aligned}
$$

onde, os valores ( $\delta=0$ e $\eta=2$ ) são associados à discretização temporal de (2.63)-(2.68) (modos altos em forma diagnostica) e os valores $(\delta=1$ e $\eta=1$ ) à discretização temporal de (2.45)-(2.50) (modos altos em forma prognóstica).

Os termos lineares no tempo $t_{n-1}$ são definidos por:

$$
\begin{aligned}
& S_{1}^{n-1}=p_{1}^{n-1}+\Delta t p_{2}^{n-1}-\Delta t p_{3 x}^{n-1}+\nu_{0} \Delta t p_{1}^{n-1} \\
& S_{2}^{n-1}=p_{2}^{n-1}-\Delta t p_{1}^{n-1}-\Delta t p_{3 y}^{n-1}+\nu_{0} \Delta t p_{2}^{n-1} \\
& S_{3}^{n-1}=p_{3}^{n-1}-\Delta t g_{0}\left(p_{1 x}^{n-1}+p_{2 y}^{n-1}\right)+\kappa_{0} \Delta t p_{3}^{n-1} \\
& S_{4}^{n-1}=q_{1}^{n-1}+\Delta t q_{2}^{n-1}-\Delta t q_{3 x}^{n-1}+\nu_{0} \Delta t q_{1}^{n-1} \\
& S_{5}^{n-1}=q_{2}^{n-1}-\Delta t q_{1}^{n-1}-\Delta t q_{3 y}^{n-1}+\nu_{0} \Delta t q_{2}^{n-1} \\
& S_{6}^{n-1}=q_{3}^{n-1}-\Delta t g_{0}\left(q_{1 x}^{n-1}+q_{2 y}^{n-1}\right)+\kappa_{0} \Delta t q_{3}^{n-1}
\end{aligned}
$$

enquanto os termos não lineares $s_{i}^{n, n+1},(i=1,2,3)$ usam os modos baixos no tempo $t_{n}$ e os modos altos no tempo $t_{n+1}$ : 


$$
\begin{aligned}
s_{1}^{n, n+1} \simeq P_{N / 2}\left(-p_{1}^{n}\left(p_{1}^{n}+q_{1}^{n+1}\right)_{x}-q_{1}^{n+1} p_{1, x}^{n}-p_{2}^{n}\left(p_{1}^{n}+q_{1}^{n+1}\right)_{y}-q_{2}^{n+1} p_{1, y}^{n}\right)(2.8 \\
s_{2}^{n, n+1} \simeq P_{N / 2}\left(-p_{1}^{n}\left(p_{2}^{n}+q_{2}^{n+1}\right)_{x}-q_{1}^{n+1} p_{2, x}^{n}-p_{2}^{n}\left(p_{2}^{n}+q_{2}^{n+1}\right)_{y}-q_{2}^{n+1} p_{2, y}^{n}\right)(2.82 \\
s_{3}^{n, n+1} \simeq P_{N / 2}\left(F-\left(p_{3}^{n}\left(p_{1}^{n}+q_{1}^{n+1}\right)\right)_{x}-\left(q_{3}^{n+1} p_{1}^{n}\right)_{x}-\left(p_{3}^{n}\left(p_{2}^{n}+q_{2}^{n+1}\right)\right)_{y}-\right. \\
\left.\quad-\left(q_{3}^{n+1} p_{2}^{n}\right)_{y}\right)
\end{aligned}
$$

Os termos não lineares $s_{i}^{n},(i=4,5,6)$ avaliam os modos baixos no tempo $t_{n}$ :

$$
\begin{aligned}
& s_{4}^{n} \simeq Q_{N}\left(-p_{1}^{n} p_{1, x}^{n}-p_{2}^{n} p_{1, y}^{n}\right) \\
& s_{5}^{n} \simeq Q_{N}\left(-p_{1}^{n} p_{2, x}^{n}-p_{2}^{n} p_{2, y}^{n}\right) \\
& s_{6}^{n} \simeq Q_{N}\left(F-\left(p_{3}^{n} p_{1}^{n}\right)_{x}-\left(p_{3}^{n} p_{2}^{n}\right)_{y}\right)
\end{aligned}
$$

Para resolver o sistema (2.69)-(2.74) transforma-se suas equações ao espaço espectral, obtendo para $(l, k) \in \mathbb{U}_{N / 2}$ o sistema matricial para os modos baixos:

$$
\left[\begin{array}{ccc}
\left(1+\nu_{0} \Delta t \lambda_{l k}\right) & -\Delta t & \mathrm{i} l \Delta t \\
\Delta t & \left(1+\nu_{0} \Delta t \lambda_{l k}\right) & \mathrm{i} k \Delta t \\
\mathrm{i} l g_{0} \Delta t & \mathrm{i} k g_{0} \Delta t & \left(1+\kappa_{0} \Delta t \lambda_{l k}\right)
\end{array}\right] \cdot\left[\begin{array}{c}
\hat{p}_{1, l k}^{n+1} \\
\hat{p}_{2, l k}^{n+1} \\
\hat{p}_{3, l k}^{n+1}
\end{array}\right]=\left[\begin{array}{c}
\hat{S}_{1, l k}^{n-1}+2 \Delta t \hat{s}_{1, l k}^{n, n+1} \\
\hat{S}_{2, l k}^{n-1}+2 \Delta t \hat{s}_{2, l k}^{, n+1} \\
\hat{S}_{3, l k}^{n-1}+2 \Delta t \hat{s}_{2, l k}^{n+n+1}
\end{array}\right]
$$

e para $(l, k) \in \mathbb{W}_{N}$ obtém-se para os modos altos:

$$
\left[\begin{array}{ccc}
\frac{\delta}{\Delta t}+\nu_{0} \lambda_{l k} & -1 & \mathrm{i} l \\
1 & \frac{\delta}{\Delta t}+\nu_{0} \lambda_{l k} & \mathrm{i} k \\
\mathrm{i} l g_{0} & \mathrm{i} k g_{0} & \frac{\delta}{\Delta t}+\kappa_{0} \lambda_{l k}
\end{array}\right] \cdot\left[\begin{array}{c}
\hat{q}_{1, l k}^{n+1} \\
\hat{q}_{2, l k}^{n+1} \\
\hat{q}_{3, l k}^{n+1}
\end{array}\right]=\left[\begin{array}{c}
\frac{\delta}{\Delta t} \hat{S}_{4, l k}^{n-1}+\frac{2}{\eta} \hat{s}_{4, l k}^{n} \\
\frac{\delta}{\Delta t} \hat{S}_{5, l k}^{n-1}+\frac{2}{\eta} \hat{s}_{5, l k}^{n} \\
\frac{\delta}{\Delta t} \hat{S}_{6, l k}^{n-1}+\frac{2}{\eta} \hat{s}_{6, l k}^{n}
\end{array}\right]
$$

Os sistemas matriciais (2.87)-(2.88) podem ser desacoplados de maneira similar ao feito no método de Galerkin linear na seção 2.1 (ver (2.32)-(2.37)).

Assumindo conhecidos $\hat{S}_{i, l k}^{n-1}, \hat{s}_{i, l k}^{n},(i=4,5,6)$ em (2.78)-(2.80) e (2.84)-(2.86) os modos altos $\hat{q}_{i, l k}^{n+1},(i=1,2,3)$ são avaliados de (2.88), estes modos altos são inseridos em (2.75)(2.77) e (2.81)-(2.83) permitindo que $\hat{S}_{i, l k}^{n}, \hat{s}_{i, l k}^{n},(i=1,2,3)$ sejam conhecidos e portanto os modos baixos $\hat{p}_{i, l k}^{n+1},(i=1,2,3)$ possam ser avaliados de (2.88). 
Resta analisar a implementação dos termos não lineares, os quais tem dois tipos de interação: modos baixos vs modos baixos e modos baixos vs modos altos. Usaremos o método das transformadas para avaliar estes termos, os produtos são avaliados no espaço físico e a seguir os coeficientes espectrais são avaliados com FFT-2D de dimensão igual à malha usada no espaço físico. O ponto crucial é tomar a malha menor possível que garanta que todos os produtos sejam avaliados sem aliasing.

$\mathrm{Na}$ iteração dos modos baixos sejam $\phi, \theta$ funções em $\mathbb{U}_{N / 2}$, logo o produto $\phi \theta$ é uma função em $\mathbb{U}_{N}$. Se usamos FFT-2D de dimensão $N \times N$ para avaliar o produto, todos seus coeficientes espectrais são avaliados corretamente, logo as projeções $P_{N / 2}(\phi \theta)$ e $Q_{N}(\phi \theta)$ são avaliadas sem aliasing.

$\mathrm{Na}$ iteração dos modos baixos e altos o produto de $\phi \in \mathbb{U}_{N / 2}$ e $\theta \in \mathbb{W}_{N}$ é uma função em $\mathbb{U}_{3 N / 2}$. Logo, se usamos a FFT-2D de dimensão $N \times N$ para avaliar $\phi \theta$, os modos extras com índices em $\left(I_{3 N / 2}-I_{N}\right)$ são absorvidos pelos modos com índices em $\left(I_{N}-I_{N / 2}\right)$, porem os modos com coeficientes em $I_{N / 2}$ estão livres de aliasing. Isto significa que o termo $P_{N / 2}(\phi \theta)$ é avaliado corretamente. Pode-se concluir que todos os termos não lineares em (2.69)-(2.74) podem ser avaliados sem aliasing desde que o método das transformadas seja aplicado com FFT-2D de dimensão $N \times N$.

\subsubsection{Implementação numérica}

Para estimar o custo computacional do Sistema (2.69)-(2.74), analisa-se sua implementação numérica, esta consiste em avaliar primeiro os modos altos no sistema (2.88) e logo os modos baixos no sistema (2.87). Para avançar à próxima iteração no tempo atualizamos os modos baixos e altos (no caso prognóstico), a seguir se mostram os detalhes desta implementação:

c1) Assuma-se conhecido os coeficientes espectrais $\hat{p}_{1, l k}^{n-1}, \hat{p}_{2, l k}^{n-1}, \hat{p}_{3, l k}^{n-1}$ para $(l, k) \in \mathbb{I}_{N / 2}$ e $p_{1}^{n}, p_{1 x}^{n}, p_{1 y}^{n}, p_{2}^{n}, p_{2 x}^{n}, p_{2 y}^{n}, p_{3}^{n}, p_{3 x}^{n}, p_{3 y}^{n}$, numa malha de dimensão $N \times N$.

c2) Avaliação dos modos altos no tempo $t_{n+1}$ : 
c2.1) Avaliar no espaço físico $s_{4}^{n}, s_{5}^{n}, s_{6}^{n}$ (definidos em (2.84)-(2.86)) na malha de dimensão $N \times N$, logo são avaliados com FFT-2D de dimensão $N \times N$ os coeficientes espectrais $\hat{s}_{4, l k}^{n}, \hat{s}_{5, l k}^{n}, \hat{s}_{6, l k}^{n}$ para $(l, k) \in \mathbb{I}_{N}$. (Neste passo são usadas 3 FFT-2D de dimensão $N \times N)$.

c2.2) Obter $\hat{q}_{i}^{n+1}(i=1, \cdots 3)$ para $(l, k) \in\left(\mathbb{I}_{N}-\mathbb{I}_{N / 2}\right)$ do sistema (2.88).

c2.3) Avaliar com FFT-2D de dimensão $N \times N$ os valores no espaço físico de $q_{i}^{n+1}$, $D_{x} q_{i}^{n+1}, D_{y} q_{i}^{n+1},(i=1, \cdots 3)$ na malha de dimensão $N \times N$. Como está se avaliando os modos altos, na avaliação das FFT-2D os coeficientes espectrais com $(l, k) \in \mathbb{I}_{N / 2}$ são completados com zeros. Aqui são usados 9 FFT-2D de tamanho $N \times N$.

c3) Avaliação dos modos baixos no tempo $t_{n+1}$ :

c3.1) Avaliar no espaço físico $s_{i}^{n, n+1}, i=1,2,3$ definidos em (2.81)-(2.83) na malha de dimensão $N \times N$. A seguir são avaliados com FFT-2D de dimensão $N \times N$ os coeficientes espectrais $\hat{s}_{1, l k}^{n, n+1}, \hat{s}_{2, l k}^{n, n+1}, \hat{s}_{3, l k}^{n, n+1}$ para $(l, k) \in \mathbb{I}_{N}$. (Neste passo são usadas 3 FFT-2D de dimensão $N \times N)$.

c3.2 Avaliar $\hat{S}_{1, l k}^{n, n+1}, \hat{S}_{2, l k}^{n, n+1}, \hat{S}_{3, l k}^{n, n+1}$ para $(l, k) \in \mathbb{I}_{N}$. a partir de (2.75)-(2.77).

c3.3 Obter $\hat{p}_{i}^{n+1} i=1,2,3$ para $(l, k) \in \mathbb{I}_{N / 2}$, do sistema (2.87).

c4) Nos passos c2 e c3 foram avaliados os modos altos e baixos no tempo $t_{n+1}$. Para avançar à próxima iteração no tempo a seguinte atualização é feita:

c4.1) Atualizar no espaço espectral: $\hat{p}_{i}^{n-1} \leftarrow \hat{p}_{1}^{n}, i=1,2,3$.

c4.2) Atualizar $\hat{p}_{i}^{n} \leftarrow \hat{p}_{2}^{n+1}, i=1,2.3$.

c4.3) Avaliar com FFT-2D de dimensão $N \times N$ os valores no espaço físico de $p_{i}^{n+1}, D_{x} p_{i}^{n+1}, D_{y} p_{i}^{n+1},(i=1, \cdots 3)$ na malha de dimensão $N \times N$. Na avaliação das FFT-2D os coeficientes espectrais $\operatorname{com}(l, k) \in \mathbb{I}_{N}-\mathbb{I}_{N / 2}$ (modos altos) são completados com zeros. Aqui usa-se usado um total de 9 FFT-2D de tamanho $N \times N$.

c5) Voltar ao passo (c2). 
Na implementação do Sistema (2.69)-(2.74) usa-se 24 FFT-2D (12 FFT-2d para os modos altos e 12 FFT-2D para os modos baixos). Assumindo que o maior trabalho é sobre a avaliação das FFT-2D, tem-se que o custo computacional de implementar (2.87)-(2.88) é de aproximadamente 24 FFT-2D de dimensão $N \times N$, a qual é da ordem $\vartheta\left(24 N^{2} \log _{2}\left(N^{2}\right)\right)$. Sendo que o custo computacional obtido da implementação do método de Galerkin linear (com mesmo número total de modos) para as equações de água rasa foi da ordem de $\vartheta\left(27 N^{2} \log _{2}\left(\frac{3 N}{2}\right)^{2}\right)$, temos uma economia computacional de aproximadamente $12 \%$ na implementação do Sistema (2.69)-(2.74).

Se os modos altos são congelados por $L$ iterações, tal como foi feito para a equação de Burgers, o custo computacional do Sistema (2.69)-(2.74) em $n$ iterações é essencialmente o da avaliação de $12 n$ FFT-2D para os modos baixos e de $12 \frac{n}{L}$ FFT-2D para os modos altos, a qual é da ordem de $\vartheta\left(12 n\left(1+\frac{1}{L}\right) N^{2} \log _{2}\left(N^{2}\right)\right)$, enquanto que o custo computacional do método de Galerkin linear é da ordem de $\vartheta\left(27 n N^{2} \log _{2}\left(\frac{3 N}{2}\right)^{2}\right)$. A economia no tempo de CPU em $n$ iterações do Sistema (2.69)-(2.74) com a avaliação dos modos altos congelados $L$ iterações é de aproximadamente $\frac{15 L-12}{27 L} \times 100 \%$ com respeito ao método de Galerkin linear. Na figura (2.19) se mostra este ganho, quando $L=1$ corresponde ao Sistema (2.69)-(2.74) sem congelar os modos altos, e conforme cresce o valor de $L$ o ganho tende a ser de $55 \%$.

\subsubsection{Resultados numéricos}

Usando as condições iniciais (2.43), a forçante $F$ e os parâmetros usados na simulação do método de Galerkin linear (ver página 80) são medidos os tempos usados na implementação dos métodos de Galerkin linear e não linear (Sistema (2.69)-(2.74)). A Figura (2.19) mostra os tempos de CPU gastos pelos distintos métodos. Na comparação temos que o método de Galerkin não linear é $12 \%$ mais rapido que o método de Galerkin linear. enquanto no caso dos modos altos congelados por 90 iterações, o tempo de CPU cai para a metade, isto é aproximadamente $50 \%$ do tempo de CPU empregado por GL: Os resultados das simulações para medir o tempo de CPU concordam com as estimativas feitas em função do número total de FFT-2D, as quais se basearam na complexidade das FFT-2D. 


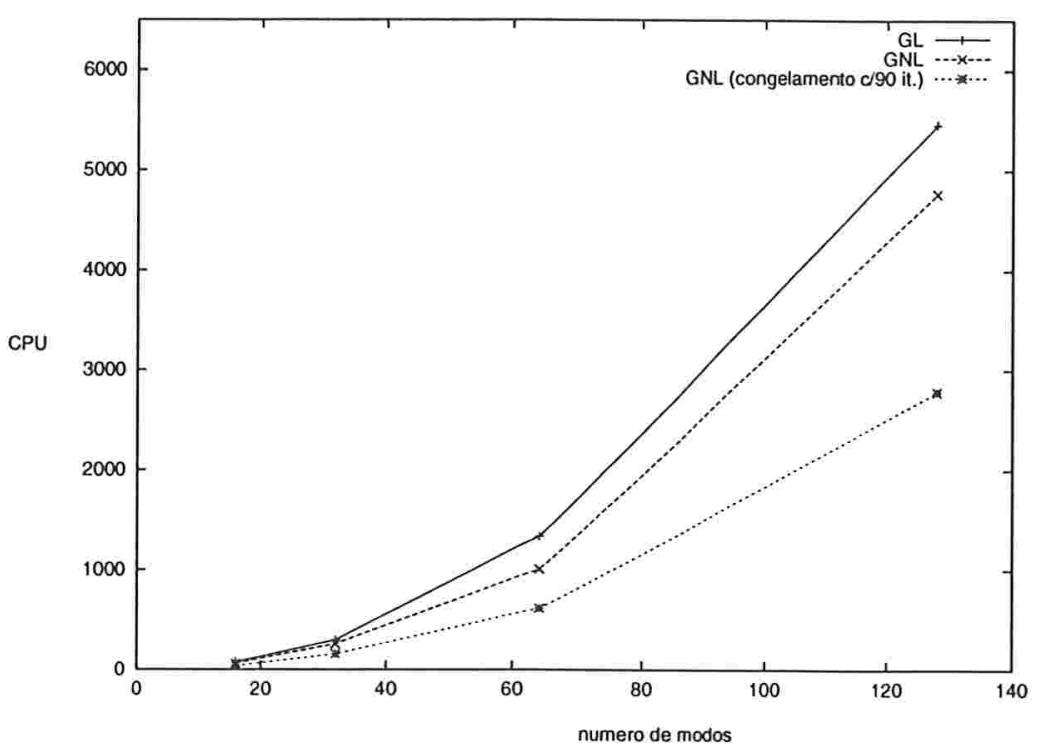

Figura 2.19: Eq. de água rasa: Tempo de CPU

Para comparar a precisão dos diferentes esquemas, estes são testados com as mesmas condições inicias (2.43). São feitas as comparações dos campos da velocidade e geopotencial para vários níveis de truncamento espacial do método de Galerkin linear e os métodos de Galerkin não linear: com e sem congelar os modos. Os gráficos foram obtidos com o uso do pacote GRADS (especial para este tipo de sistemas meteorológicos). Nas Figuras 2.21-2.26 se apresentam os resultados dos métodos de Galerkin para os campos $u, v$, e $z \operatorname{com} \Delta t=9$ min. e 200 iterações no tempo, o qual equivale a $t=30$ horas de prognóstico. Os campos são apresentados na sua forma dimensionalizada.

A parte (a) de cada figura compara o método de Galerkin linear para $P_{N}$ e $P_{2 N}$ com $N=8,16,32$. Nos partes (b), (c) e (d) compara-se o método de Galerkin Linear para $P_{N}$ e o método de Galerkin não linear para $P_{N / 2}+Q_{N} \operatorname{com} N=16$, 32. Na parte (b) os modos altos são avaliados de forma diagnóstica para o método de Galerkin não linear, na parte (c) também é usado o Sistema (2.69)-(2.74) com os modos altos congelados durante cada 90 iterações, enquanto na parte (d) os modos altos são avaliados em forma prognóstica). Os resultados mostram que a precisão do método de Galerkin não linear com a projeção $P_{N / 2}+Q_{N / 2}$ é intermediária entre a do método de GL com a projeção $P_{N / 2}$ e a do método de GL com a projeção $P_{N}$, ficando muito mais próxima desta última, 
mais precisa. Isto é, o método de Galerkin não linear com a projeção $P_{N / 2}+Q_{N / 2}$ tem precisão próxima ao do método de Galerkin linear com a projeção $P_{N}$, a um custo computacional menor (especialmente se o congelamento dos modos altos for ativado). Para nossa escolha de forçante e condição inicial não foram observadas diferenças significativas entre as partes (b) e (c), isto é, pode-se congelar os modos altos sem perda significativa da precisão. As diferenças entre o uso de GNL na forma prognóstica ou diagnóstica não chegam a ser significativas.

Um outro teste é feito, ao sistema anterior é adicionada uma forçante $F_{1}(x, y)=\delta_{r}(x) \delta_{r}(y)$, onde: $\quad \delta_{r}=\left\{\begin{array}{ll}\frac{r}{4}(1 .+\cos (r s+\pi)), & |s| \leq \pi(1+r) \\ 0, & \text { em outro caso }\end{array}\right.$.

Esta forçante localizada no centro da malha aproxima a função delta de Dirac quan-

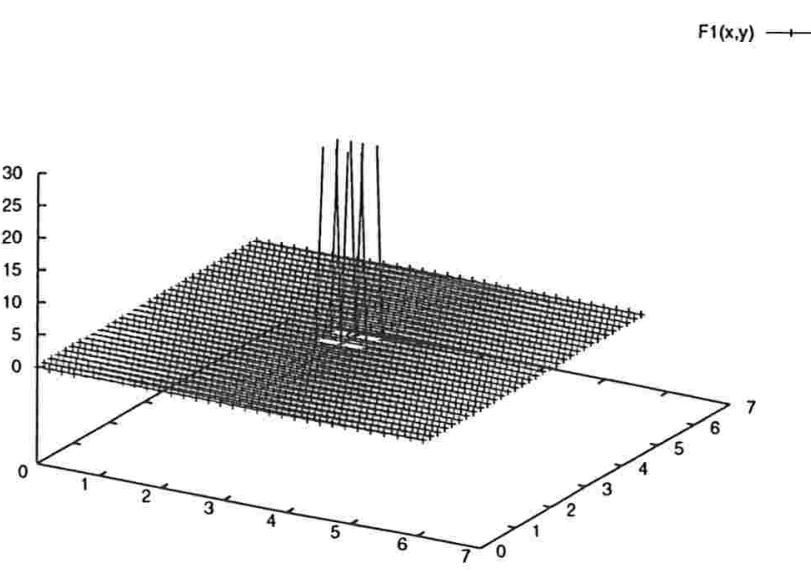

Figura 2.20: Eq. de água rasa: Forçante $F_{1}$

do $r$ cresce sem limite (na figura (2.20) é mostrada esta forçante quando $r=20$ ). $\mathrm{O}$ sistema anterior é assim perturbado com $F_{1}$ atuando no centro da malha. Gráficos da evolução dos campos da velocidade e do geopotencial para vários valores do tempo $t$ são apresentados nas figuras (2.27)-(2.29), onde também pode observar-se que o divergente do campo das velocidades mantém-se pequeno. Uma comparação entre os métodos de Galerkin linear e não linear para diversos níveis de truncamento mostra novamente o mesmo comportamento descrito anteriormente (ver figuras (2.30)-(2.32)). 


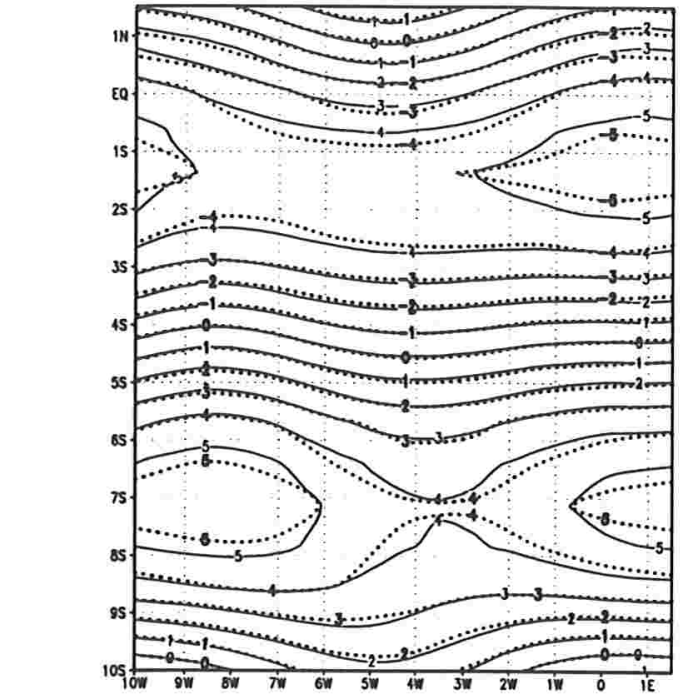

aros: counces

(o)

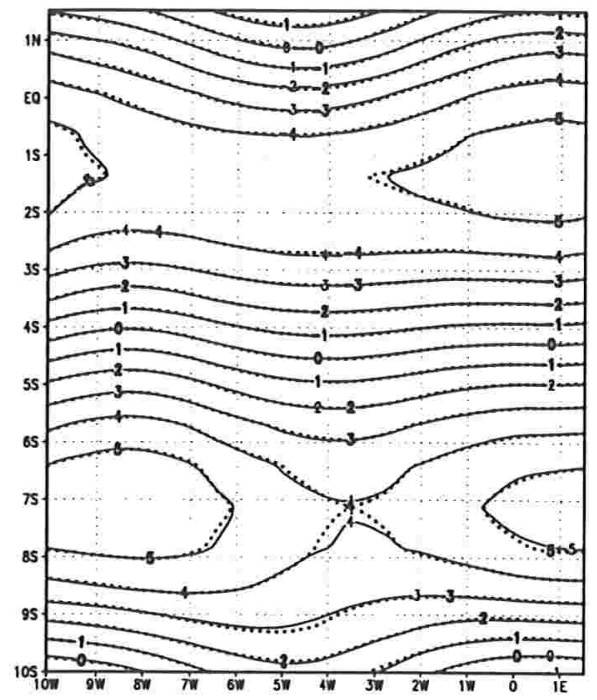

(c)

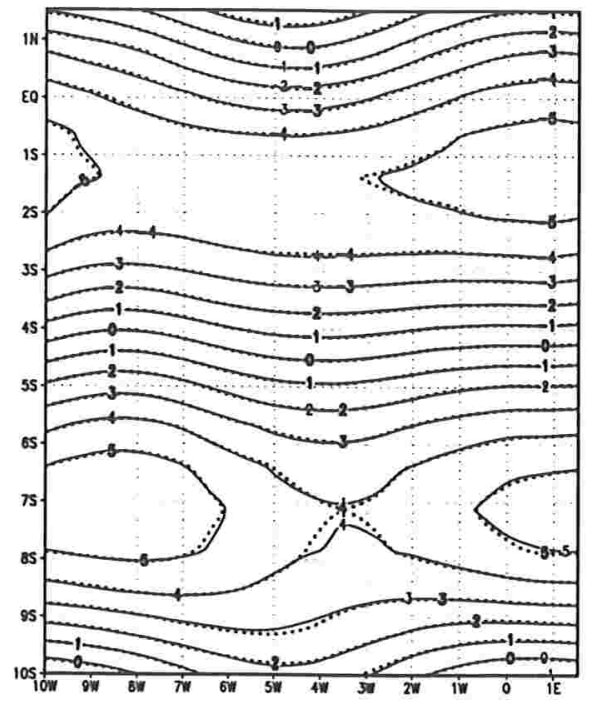

(b)

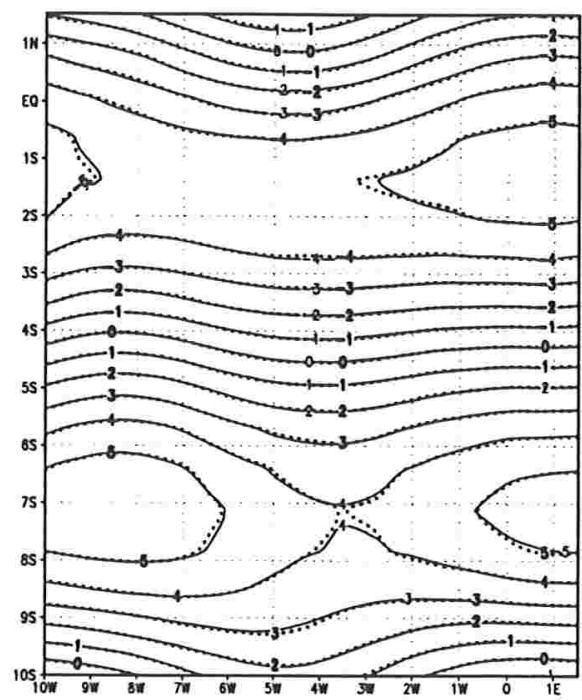

(d)

Figura 2.21: Eq. de água rasa (velocidade $u$ ): GL vs GNL com 16 modos
(a) -u (GL: $\left.P_{16}\right) ; \cdots$ u (Gl: $\left.P_{8}\right)$;
(b) -u (GL: $\left.P_{16}\right)$;
$\cdots \mathrm{u}\left(\mathrm{Gnl}: P_{8}+Q_{16}\right)$;
(c) $-\mathrm{u}\left(\mathrm{GL}: P_{16}\right)$;

$\cdots \mathrm{u}\left(\mathrm{Gnl}: P_{8}+Q_{16}\right)$ (modos altos avaliados c/ 90 iter.);

(d) -u (GL: $\left.P_{16}\right)$;

$\cdots$ u $\left(\mathrm{Gnl}: P_{8}+Q_{16}\right)$

(modos altos avaliados em forma prognóstica). $\Delta t=9 \mathrm{~min}$. 


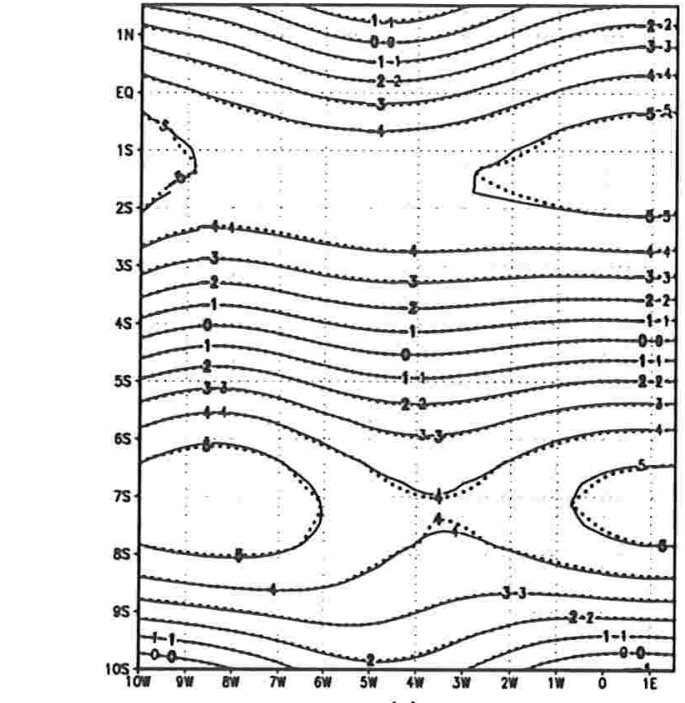

QHos: canroes

(a)

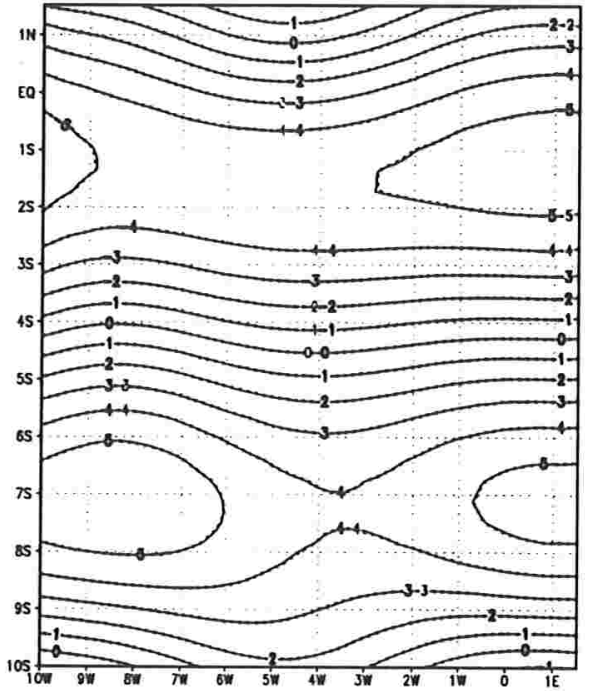

(c)

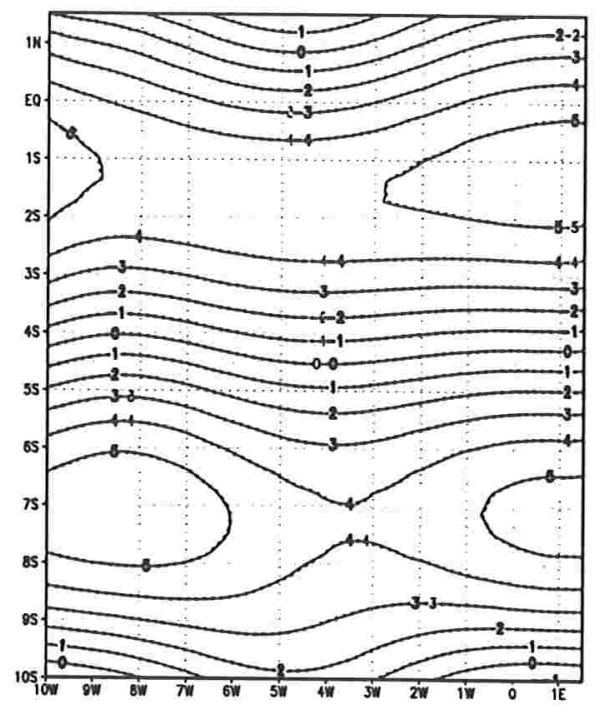

(b)

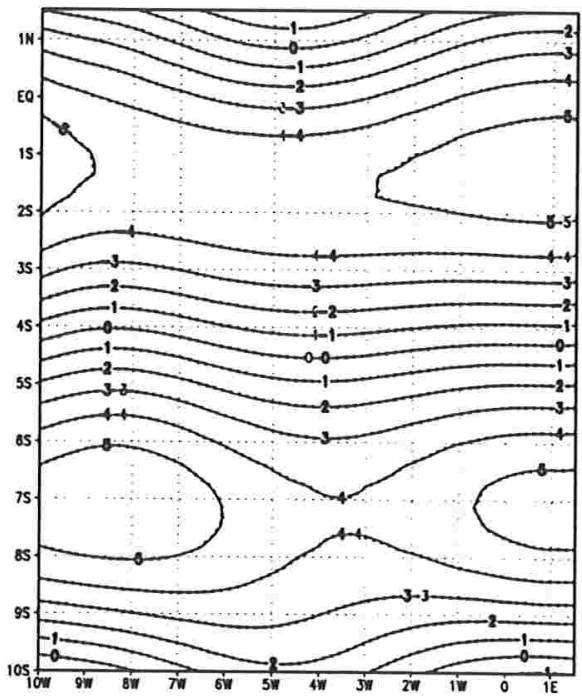

(d)

Figura 2.22: Eq. de água rasa (velocidade $u$ ): GL vs GNL com 32 modos

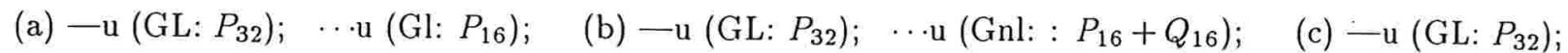
$\cdots \mathrm{u}\left(\mathrm{Gnl}: P_{16}+Q_{16}\right)$ (modos altos avaliados c/ 90 iter.); $\quad$ (d) $-\mathrm{u}\left(\mathrm{GL}: P_{32}\right) ; \cdots \mathrm{u}\left(\mathrm{Gnl}: P_{16}+Q_{16}\right)$ (modos altos avaliados em forma prognóstica). $\Delta t=9 \mathrm{~min}$. 

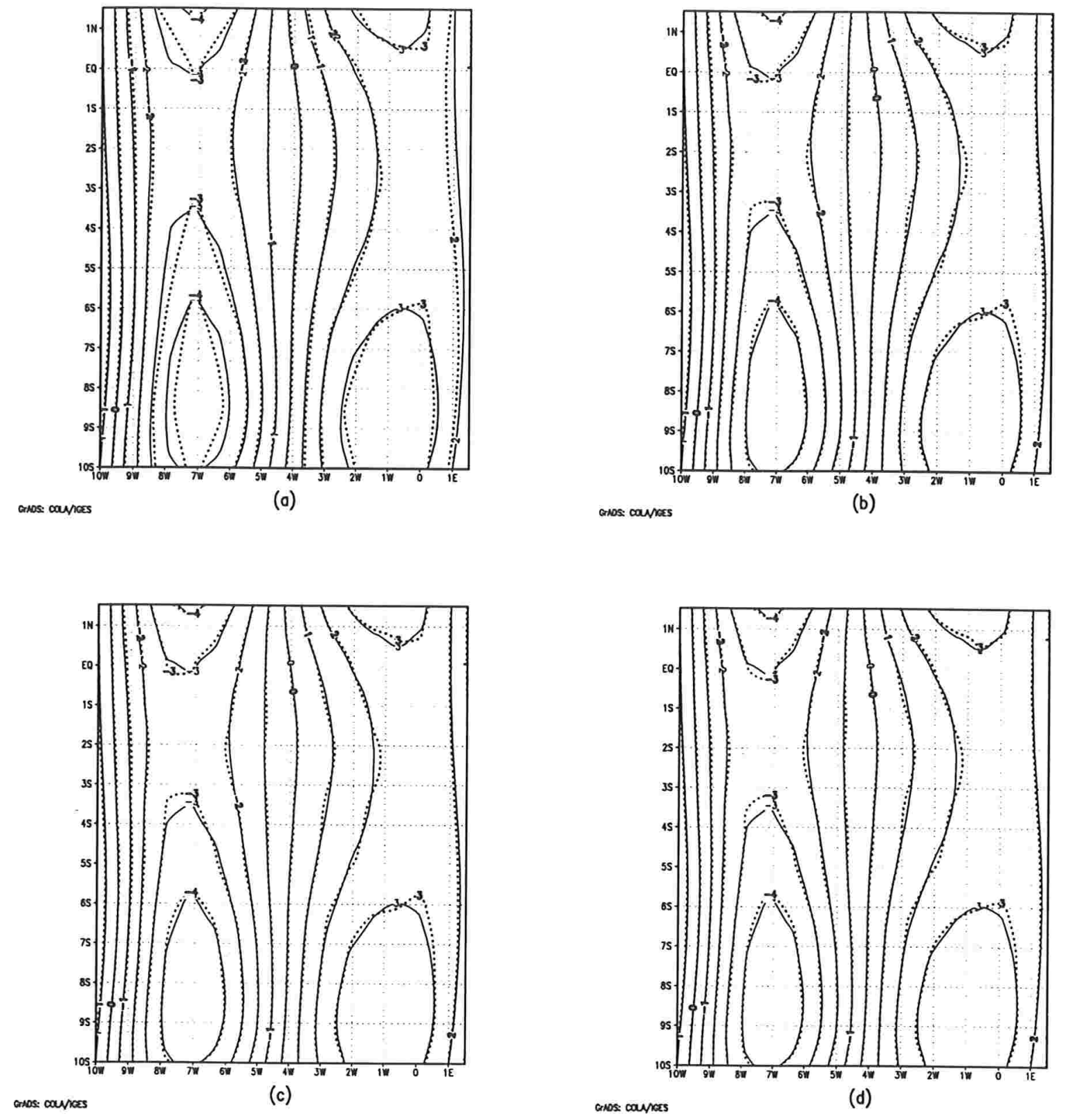

Figura 2.23: Eq. de água rasa (velocidade $v$ ): GL vs GNL com 16 modos
(a) -v (GL: 16 modos); $\cdots v$ (Gl: 8 modos);
(b) $-\mathrm{v}\left(\mathrm{GL}: P_{16}\right)$;
$\cdots \mathrm{v}\left(\mathrm{Gnl}: P_{8}+Q_{8}\right)$
(c) $-\mathrm{v}$

(GL: $\left.P_{16}\right) ; \cdots v\left(G n l: P_{8}+Q_{8}\right)$ (modos altos avaliados c/ 90 iter.); $\quad$ (d) $-\mathrm{v}\left(\mathrm{GL}: P_{16}\right) ; \cdots \mathrm{v}$ (Gnl: $\left.P_{8}+Q_{8}\right)$ (modos altos avaliados em forma prognóstica). $\Delta t=9 \mathrm{~min}$. 

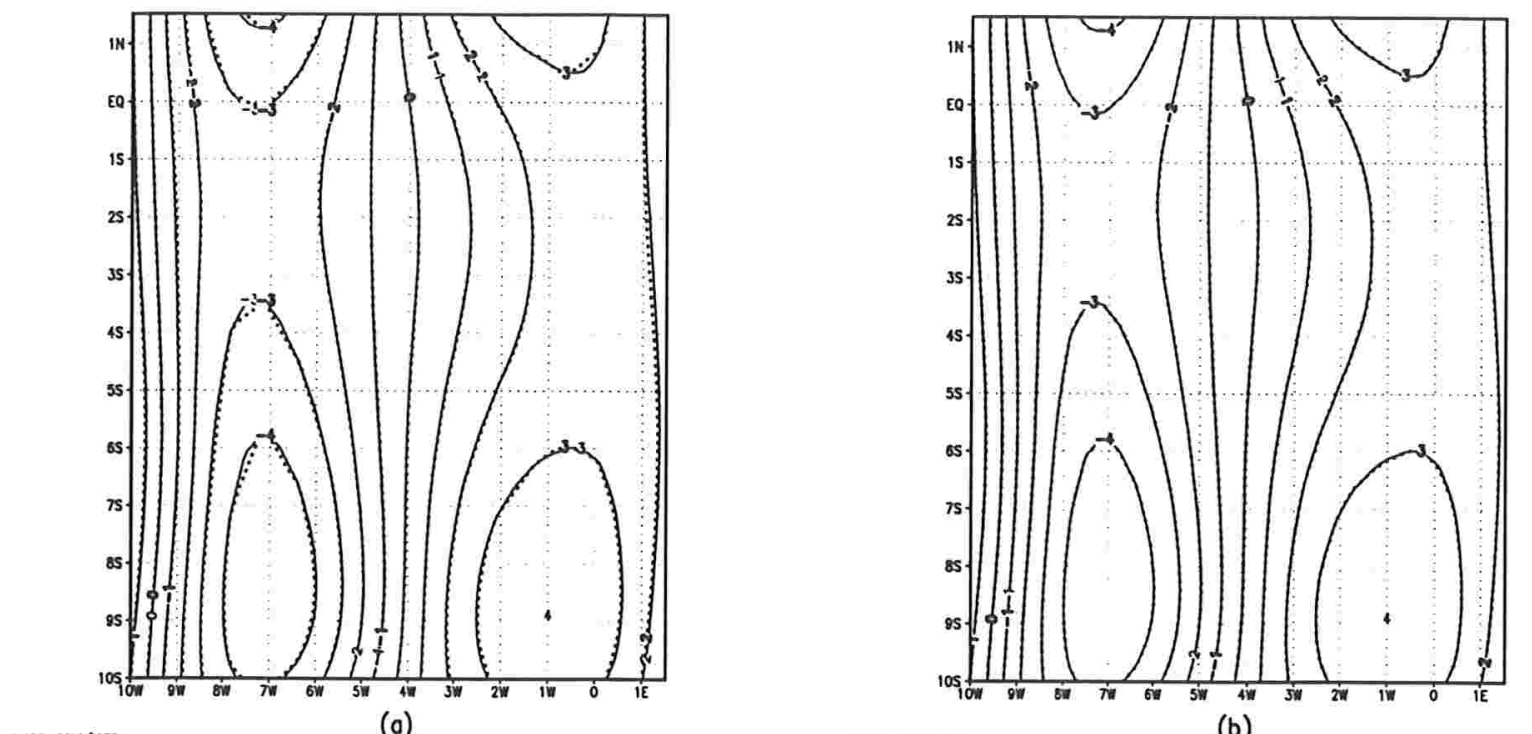

aros: cavioes

(o)

anos cannoss

(b)
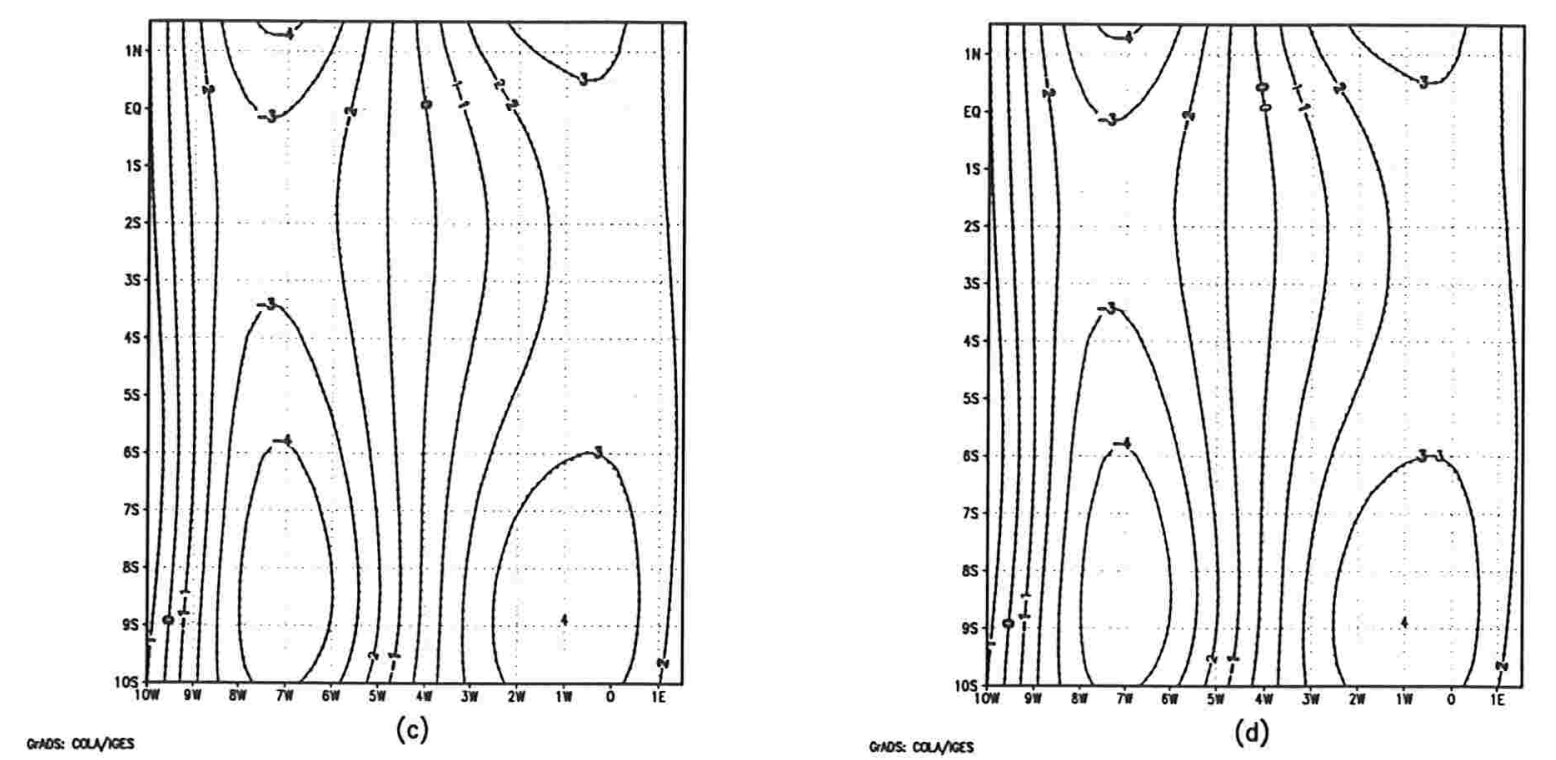

Figura 2.24: Eq. de água rasa (velocidade $v$ ): GL vs GNL com 32 modos (a) —v (GL: 32 modos); $\cdots \mathrm{v}$ (Gl: 16 modos); (b) $-\mathrm{v}\left(\mathrm{GL}: P_{32}\right) ; \cdots \mathrm{v}\left(\mathrm{Gnl}: P_{16}+Q_{16}\right) ; \quad$ (c) $-\mathrm{v}$ (GL: $\left.P_{32}\right) ; \cdots v\left(G n l: P_{16}+Q_{16}\right)$ (modos altos avaliados c/ 90 iter.); $\quad(\mathrm{d})-\mathrm{v}\left(\mathrm{GL}: P_{32}\right) ; \cdots \mathrm{v}(\mathrm{Gnl}$ : $\left.P_{16}+Q_{16}\right)$ (modos altos avaliados em forma prognóstica). $\Delta t=9 \mathrm{~min}$. 


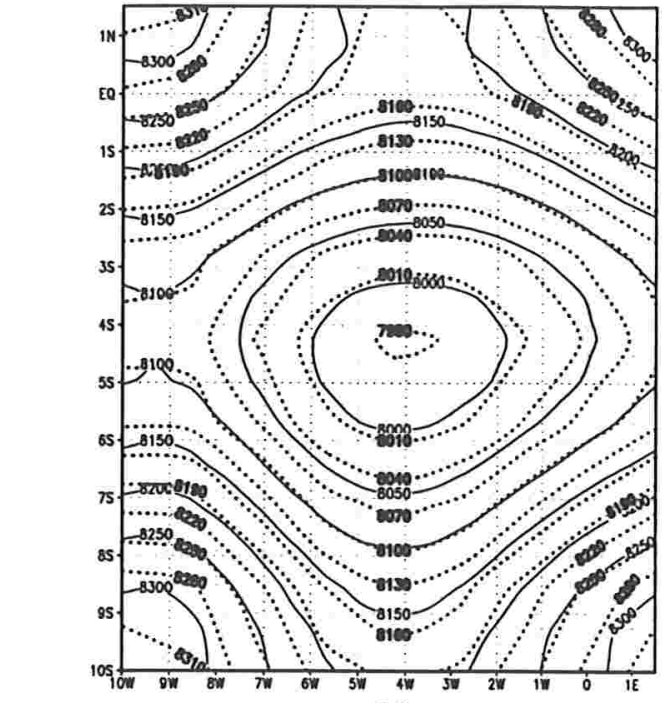

anos cavioss

(a)

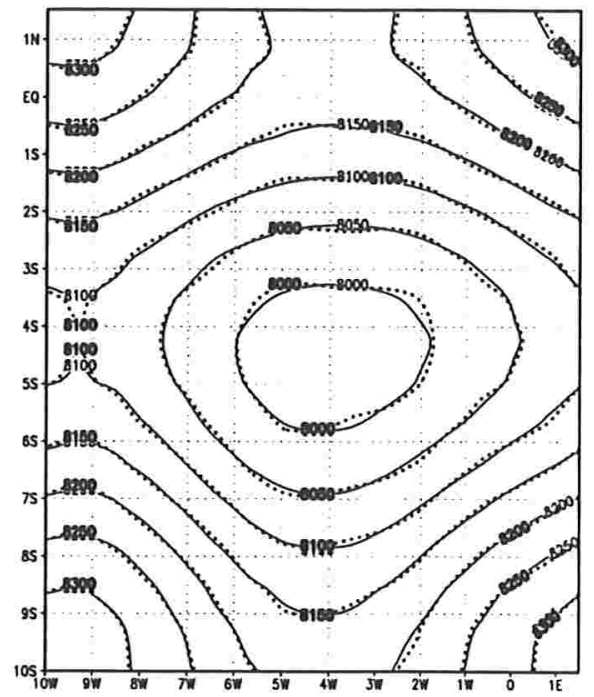

(c)

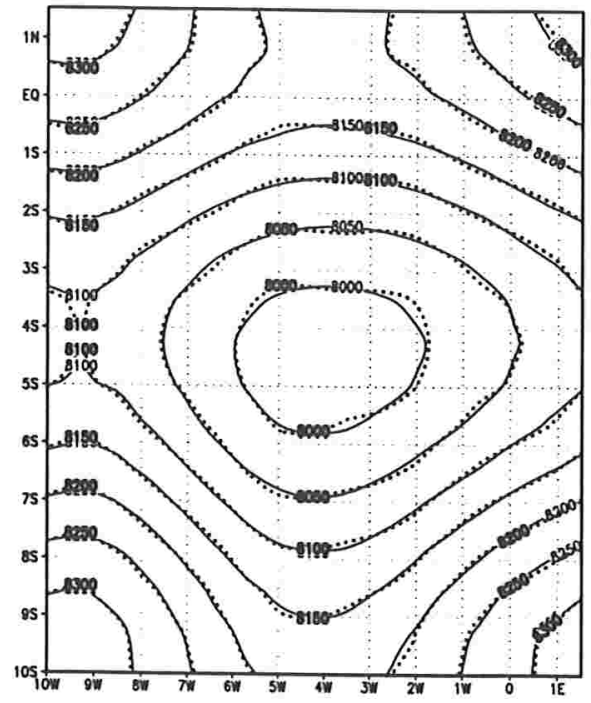

(b)

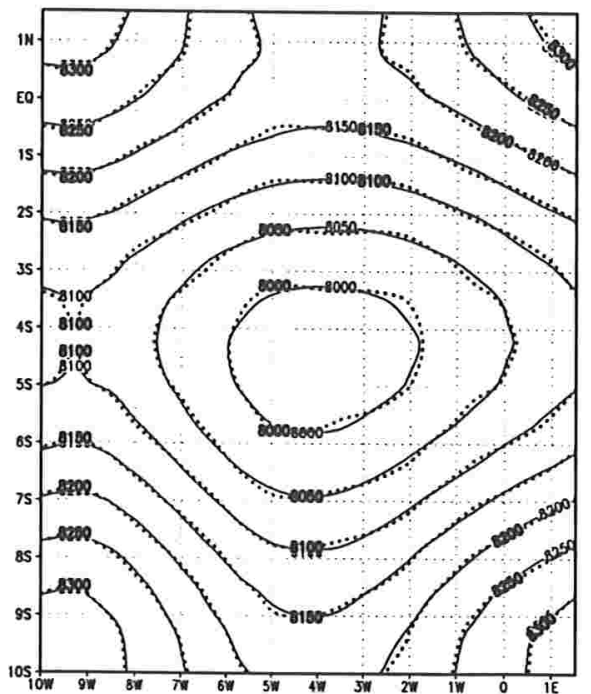

(d)

Figura 2.25: Eq. de água rasa (geopotencial $z+h$ ): GL vs GNL com 16 modos

(a) $-\mathrm{z}+\mathrm{h}\left(\mathrm{GL}: 16\right.$ modos); $\cdots \mathrm{z}+\mathrm{h}\left(\mathrm{Gl}: 8\right.$ modos); $\quad$ (b) $-\mathrm{z}+\mathrm{h}\left(\mathrm{GL}: P_{16}\right) ; \cdots \mathrm{z}+\mathrm{h}\left(\mathrm{Gnl}: P_{8}+Q_{8}\right)$;

(c) $-\mathrm{z}+\mathrm{h}\left(\mathrm{GL}: P_{16}\right) ; \quad \cdots \mathrm{z}+\mathrm{h}\left(\mathrm{Gnl}: P_{8}+Q_{8}\right)(\operatorname{modos}$ altos avaliados $\mathrm{c} / 90$ iter.); $\quad$ (d) - $\mathrm{z}+\mathrm{h}(\mathrm{GL}$ :

$\left.P_{16}\right) ; \cdots \mathrm{z}+\mathrm{h}\left(\mathrm{Gnl}: P_{8}+Q_{8}\right)$ (modos altos avaliados em forma prognóstica). $\Delta t=9 \mathrm{~min}$. 


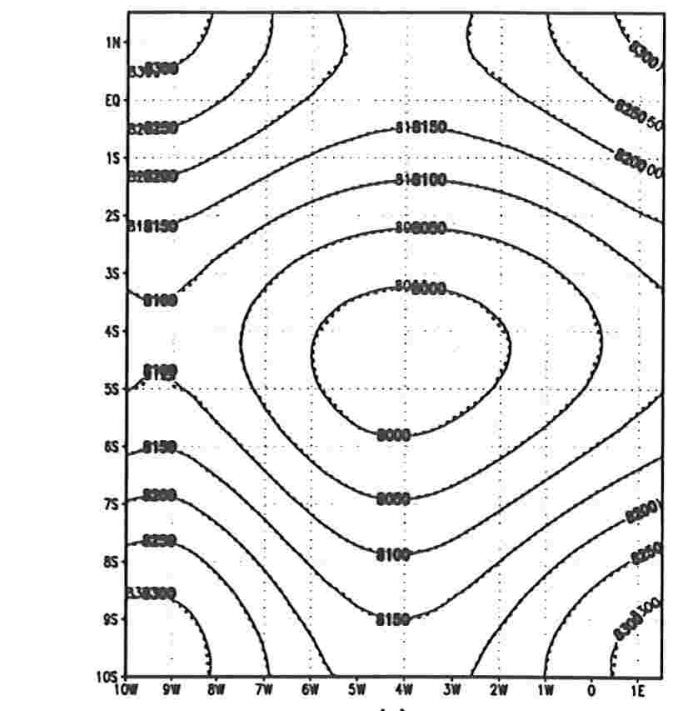

anos: caunoss

(a)

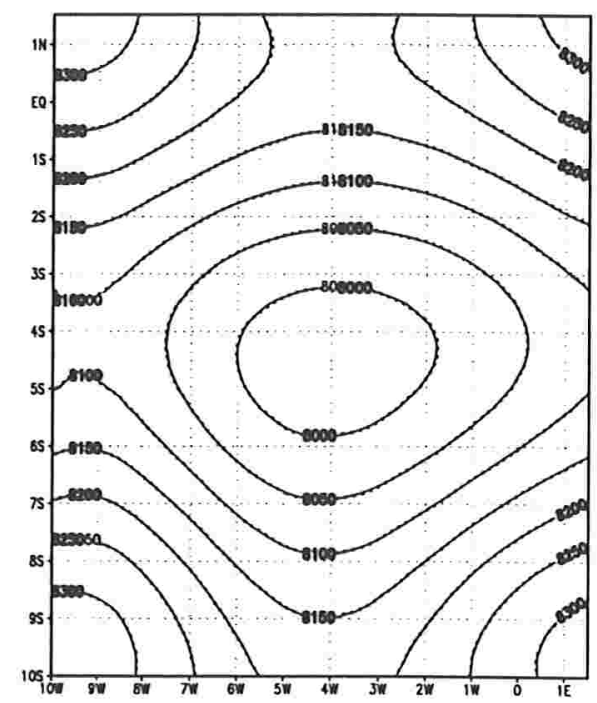

(c)

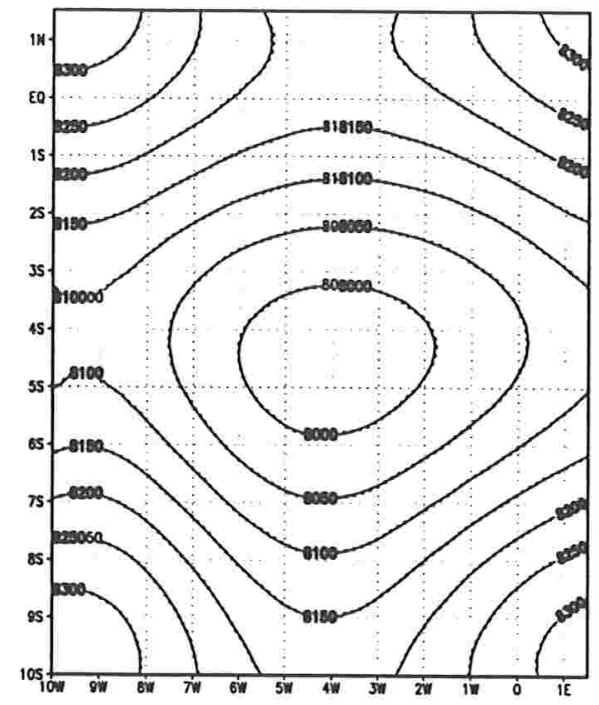

(b)

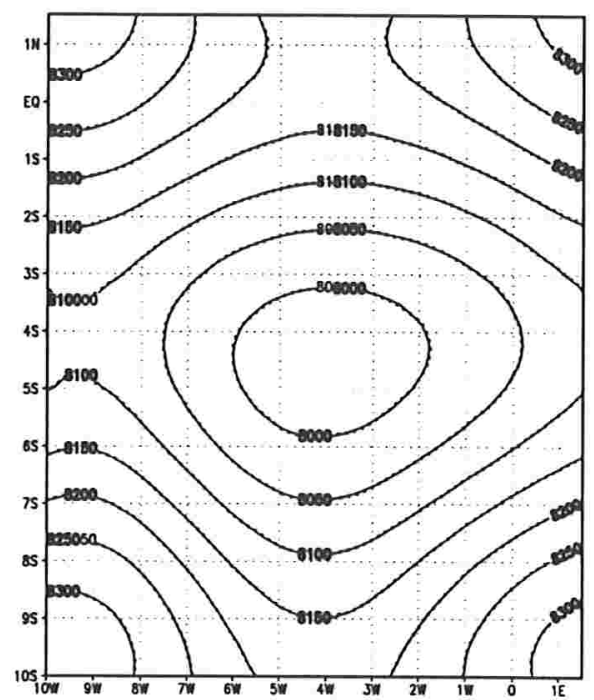

(d)

Figura 2.26: Eq. de água rasa (geopotencial $z+h$ ): GL vs GNL com 32 modos

(a) - $\mathrm{z}+\mathrm{h}$ (GL: 32 modos); $\cdots \mathrm{z}+\mathrm{h}\left(\mathrm{Gl}: 16\right.$ modos); $\quad$ (b) $-\mathrm{z}+\mathrm{h}\left(\mathrm{GL}: P_{32}\right) ; \cdots \mathrm{z}+\mathrm{h}\left(\mathrm{Gnil}: P_{16}+Q_{16}\right)$;

(c) $-\mathrm{z}+\mathrm{h}\left(\mathrm{GL}: P_{32}\right) ; \cdots \mathrm{z}+\mathrm{h}\left(\mathrm{Gnl}: P_{16}+Q_{16}\right)$ (modos altos avaliados $\mathrm{c} / 90$ iter.); $\quad$ (d) $-\mathrm{z}+\mathrm{h}(\mathrm{GL}$ : $\left.P_{32}\right) ; \cdots \mathrm{z}+\mathrm{h}\left(\mathrm{Gnl}: P_{16}+Q_{16}\right)$ (modos altos avaliados em forma prognóstica). $\Delta t=9 \mathrm{~min}$. 


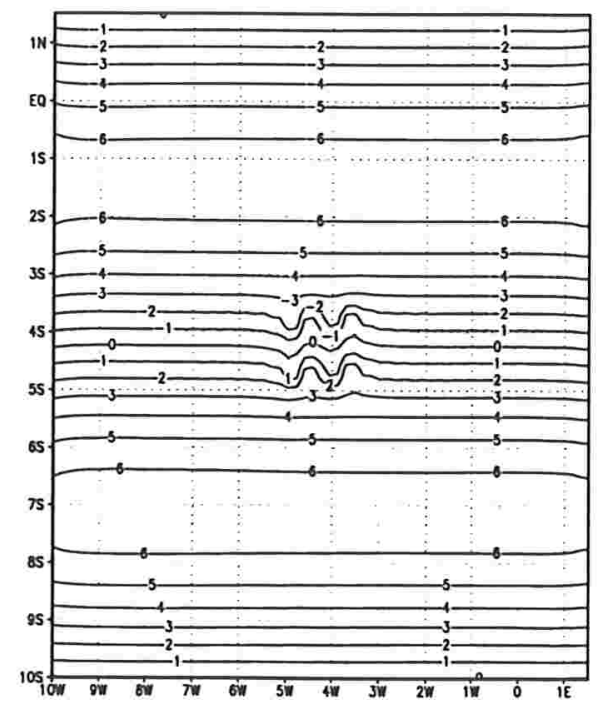

(o)

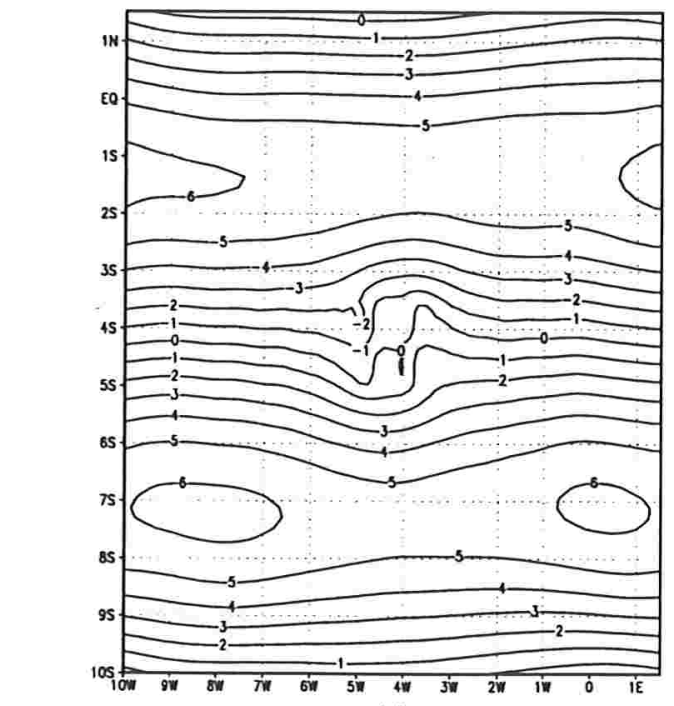

(c)

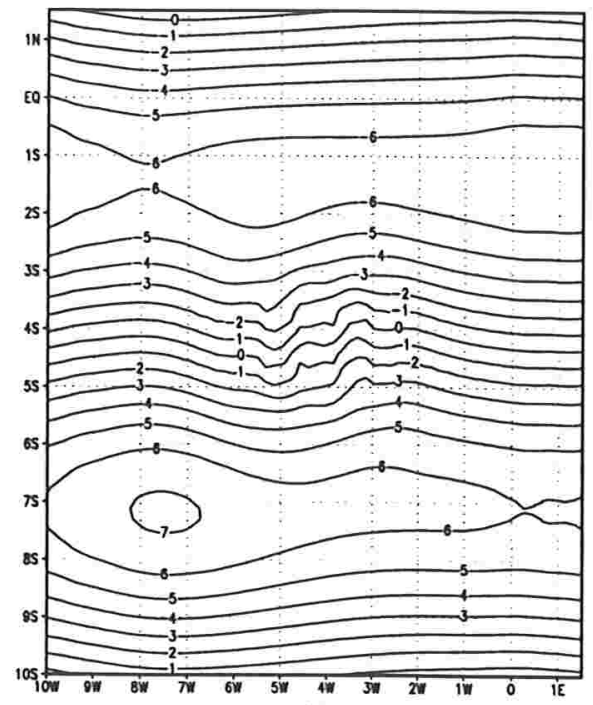

(b)

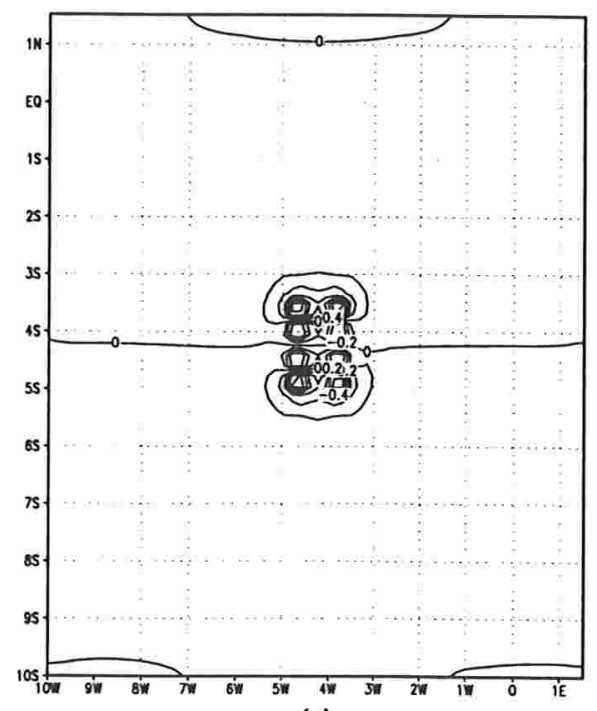

(d)

Figura 2.27: Eq. de água rasa com forçante $F_{1}(u$ e $v)$ : GL com 64 modos
(a) $\mathrm{u}(\mathrm{t}=18 \mathrm{~min})$,
(b) $u(t=1.5 \mathrm{~h})$,
(c) $\mathrm{u}(\mathrm{t}=6 \mathrm{~h})$,
(d) $\mathrm{v}(\mathrm{t}=18 \mathrm{~min})$ 

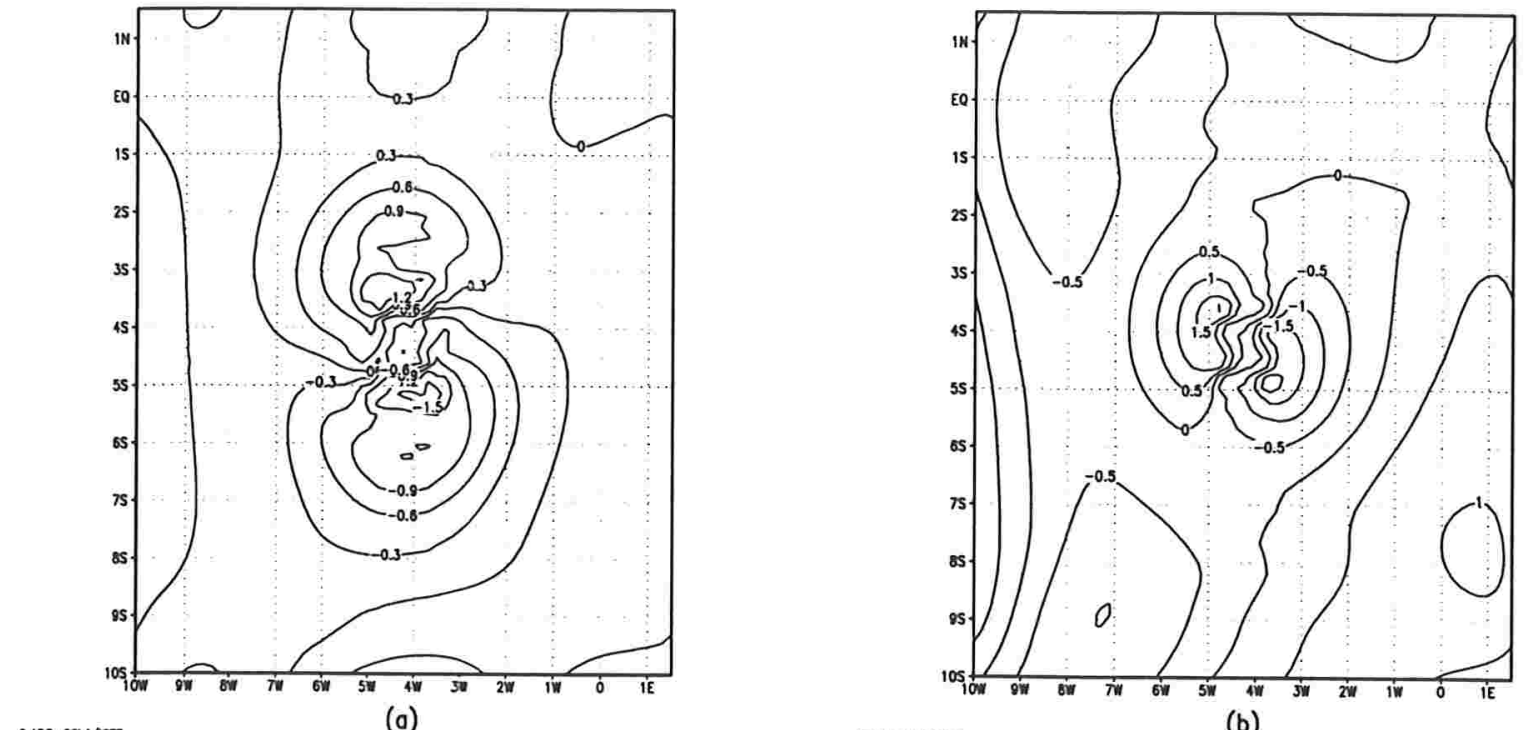

anos: cavioes

(a)

anos: caunos

(b)

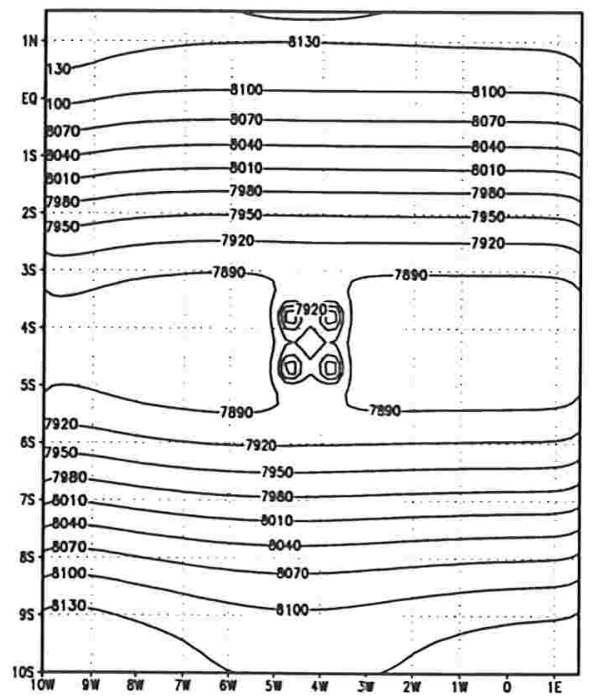

(c)

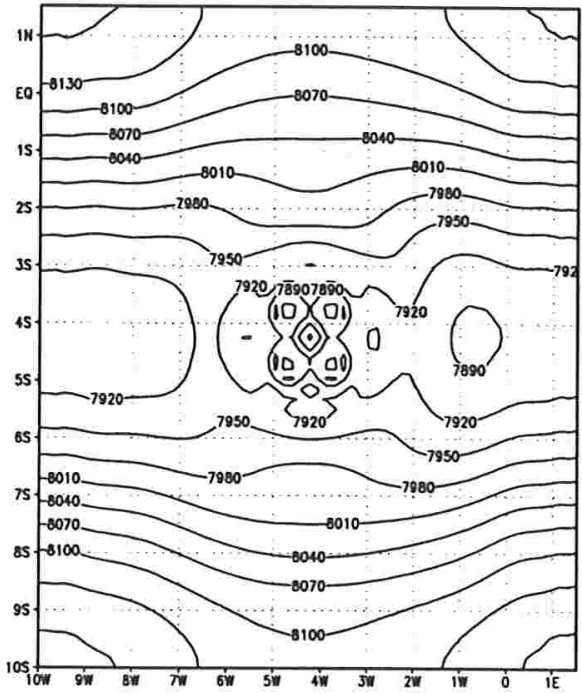

(d)

cros: caunces

Figura 2.28: Eq. de água rasa com forçante $F_{1}(v$ e $z+h)$ : GL com 64 modos
(a) $\mathrm{v}(\mathrm{t}=1.5 \mathrm{~h})$,
(b) $v(t=6 h)$,
(c) $\mathrm{z}+\mathrm{h}(\mathrm{t}=18 \mathrm{~min})$,
(d) $\mathrm{z}+\mathrm{h}(\mathrm{t}=1.5 \mathrm{~h})$ 


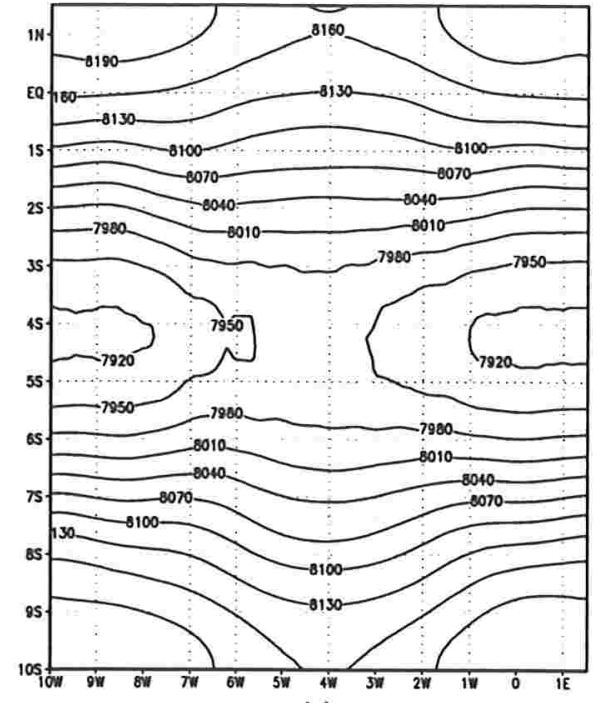

GNos: councess

(a)

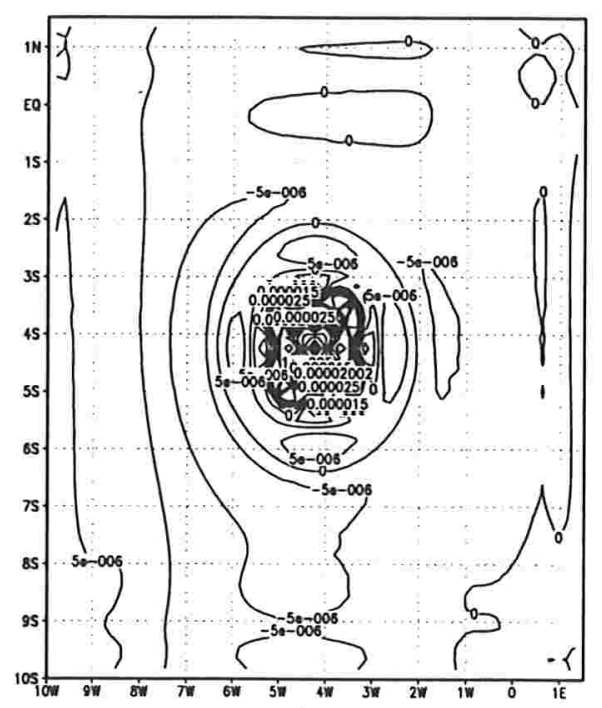

(c)

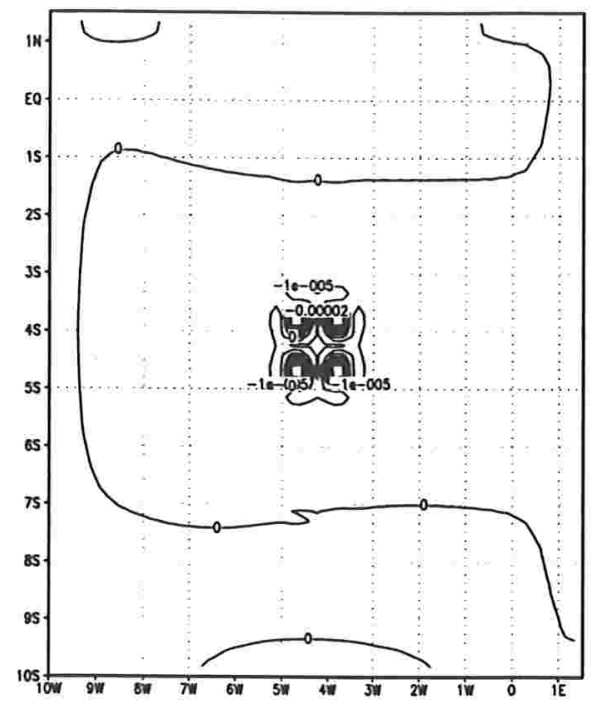

(b)

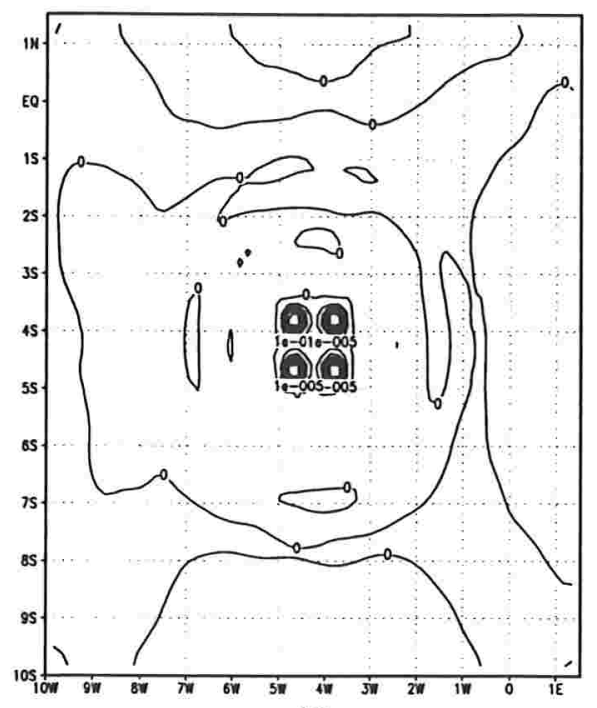

(d)

Figura 2.29: Eq. de água rasa com forçante $F_{1}$ (geopotencial $z+h$ e div): GL com 64 modos
(a) $\mathrm{z}+\mathrm{h}(\mathrm{t}=6 \mathrm{~h})$,
(b) $\operatorname{div}(\mathrm{t}=18 \mathrm{~min})$,
(c) $\operatorname{div}(\mathrm{t}=1.5 \mathrm{~h})$,
(d) $\operatorname{div}(t=6 \mathrm{~h})$ 

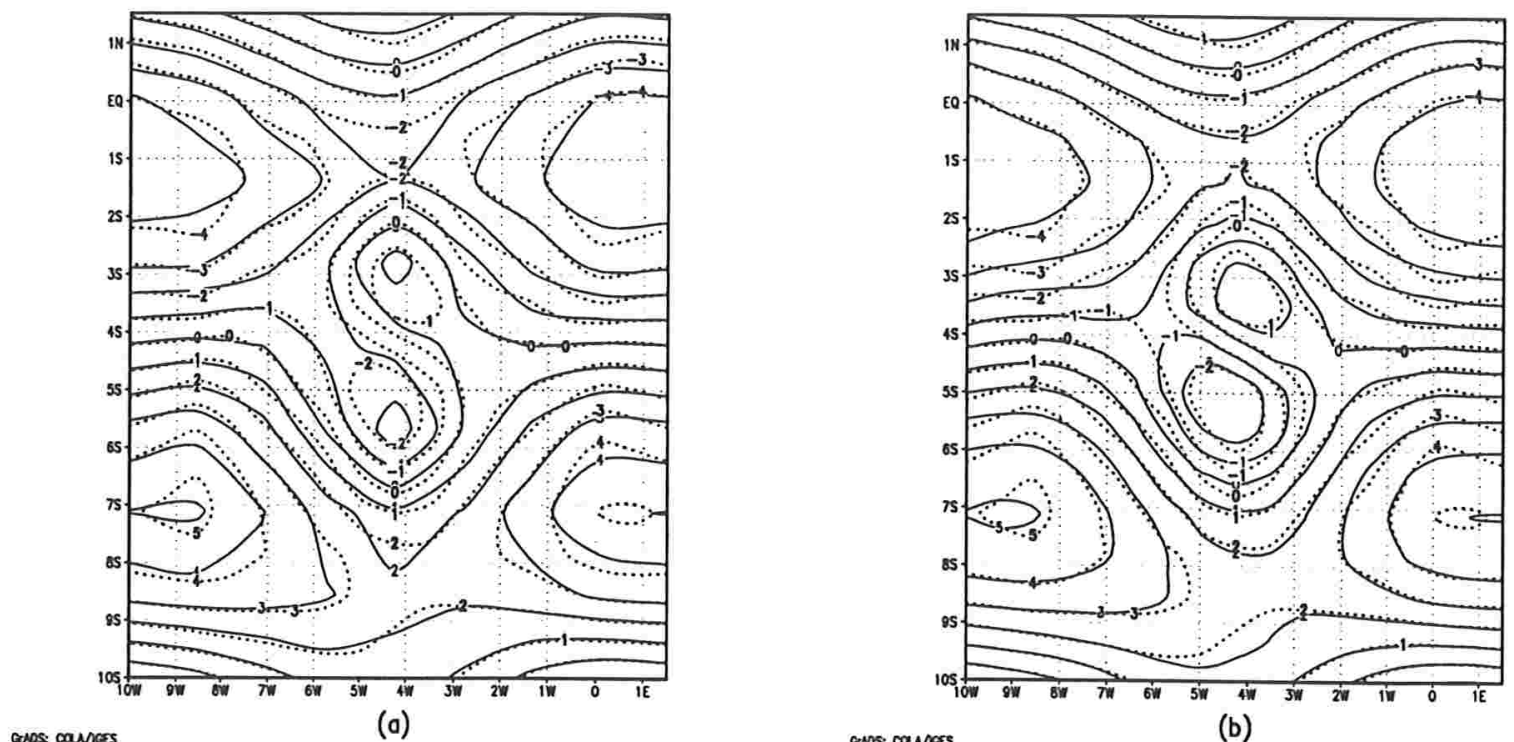

(a)

anos: cannoss

(b)

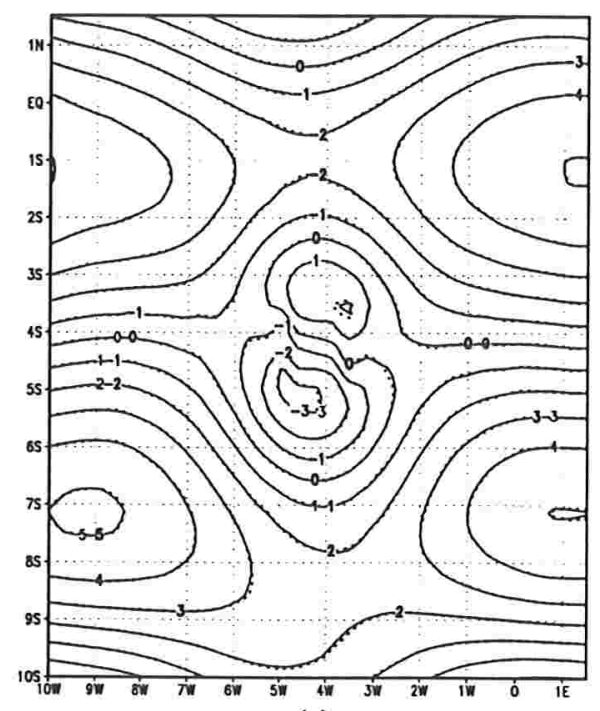

(c)

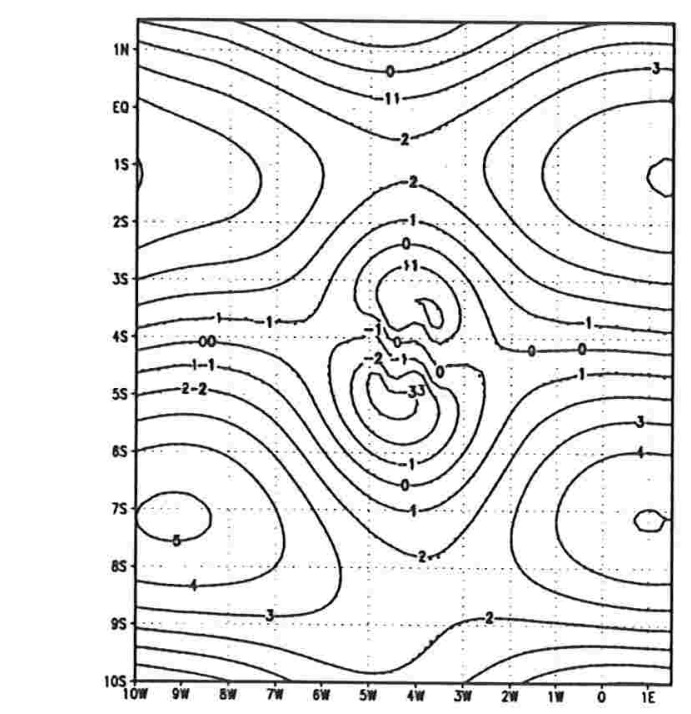

gros: couroes

(d)

Figura 2.30: Eq. de água rasa com forçante $F_{1}$ (velocidade $u$ ): GL vs GNL (c/ 90 iter.)
(a) -u (GL: $\left.P_{8}\right) ; \cdots u\left(\right.$ Gnl: $\left.P_{8}+Q_{16}\right)$;
(b) $-\mathrm{u}\left(\mathrm{GL}: P_{16}\right) ; \cdots \mathrm{u}\left(\mathrm{Gnl}: P_{8}+Q_{16}\right)$;
(c) $-\mathrm{u}$ (GL:

$\left.P_{32}\right) ; \cdots$ u $\left(\right.$ Gnl: $\left.P_{16}+Q_{32}\right) ; \quad\left(\right.$ d) - u $\left(\right.$ GL: $\left.P_{64}\right) ; \cdots$ u $\left(\right.$ Gnl: $\left.P_{32}+Q_{64}\right) \quad \Delta t=9$ min. 

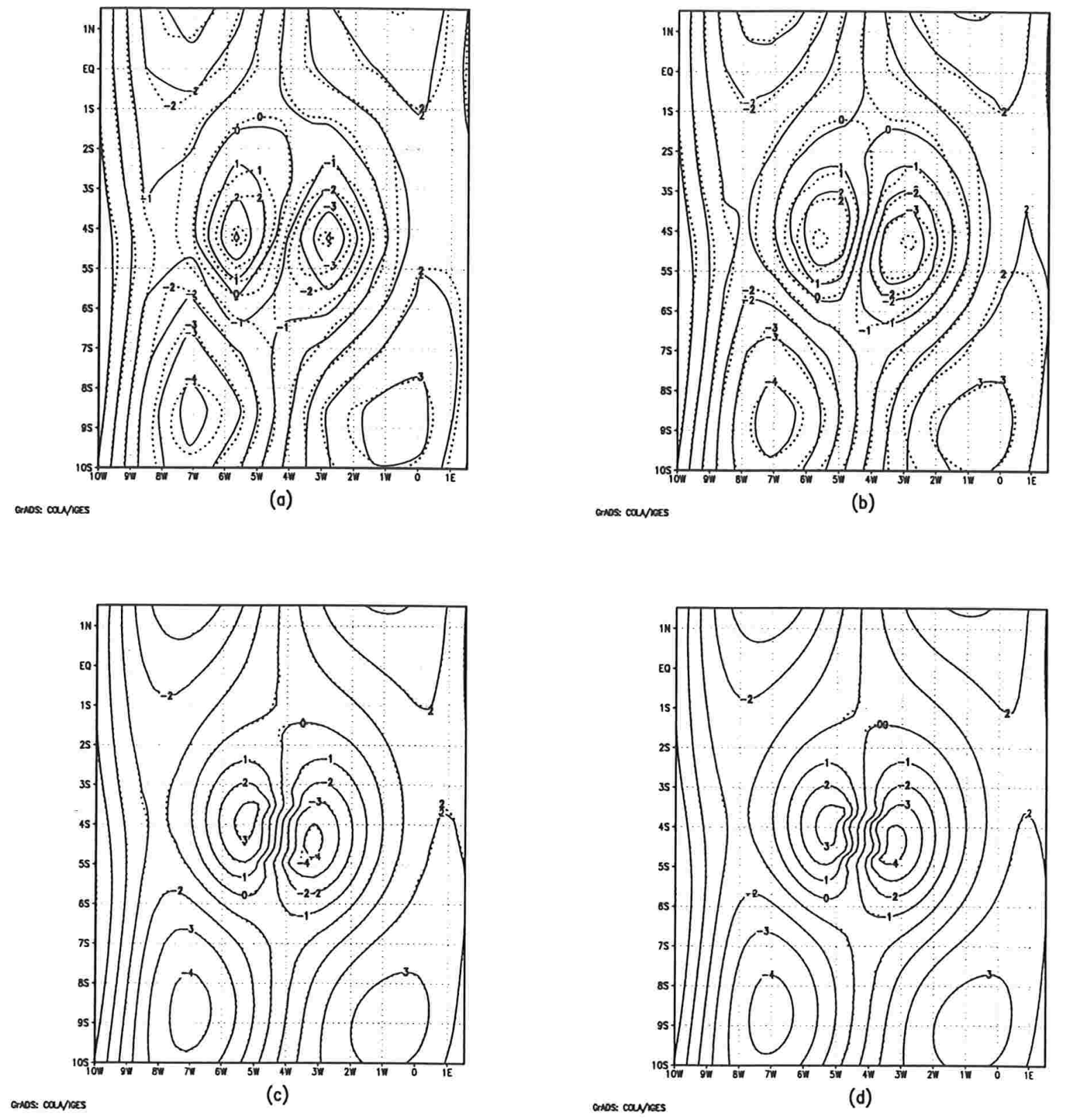

Figura 2.31: Eq. de água rasa com forçante $F_{1}$ (velocidade v): GL vs GNL (c/ 90 iter.) (a) $-\mathrm{v}\left(\mathrm{GL}: P_{8}\right) ; \cdots \mathrm{v}\left(\mathrm{Gnl}: P_{8}+Q_{16}\right) ; \quad$ (b) $-\mathrm{v}\left(\mathrm{GL}: P_{16}\right) ; \cdots \mathrm{v}\left(\mathrm{Gnl}: P_{8}+Q_{16}\right) ; \quad$ (c) $-\mathrm{v}(\mathrm{GL}:$ $\left.P_{32}\right) ; \quad \cdots \mathrm{v}\left(\mathrm{Gnl}: P_{16}+Q_{32}\right) ; \quad(\mathrm{d})-\mathrm{v}\left(\mathrm{GL}: P_{64}\right) ; \cdots \mathrm{v}\left(\mathrm{Gnl}: P_{32}+Q_{64}\right) \quad \Delta t=9 \mathrm{~min}$. 


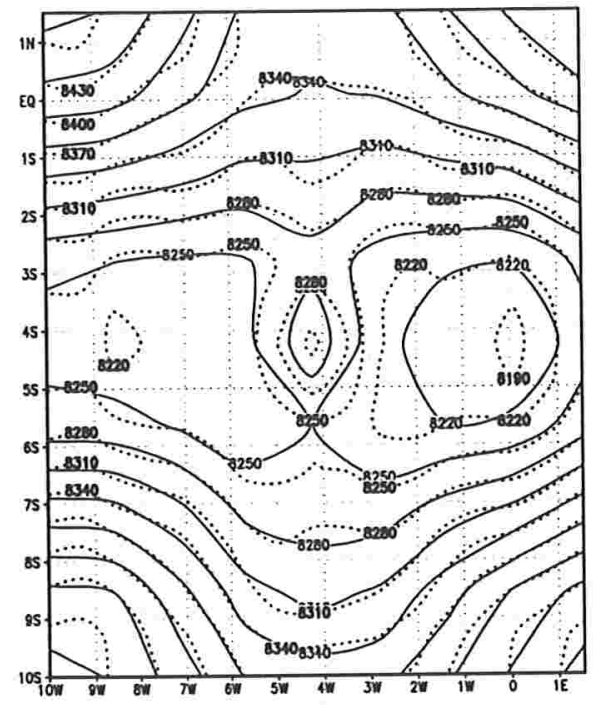

Gros: counoes

(a)

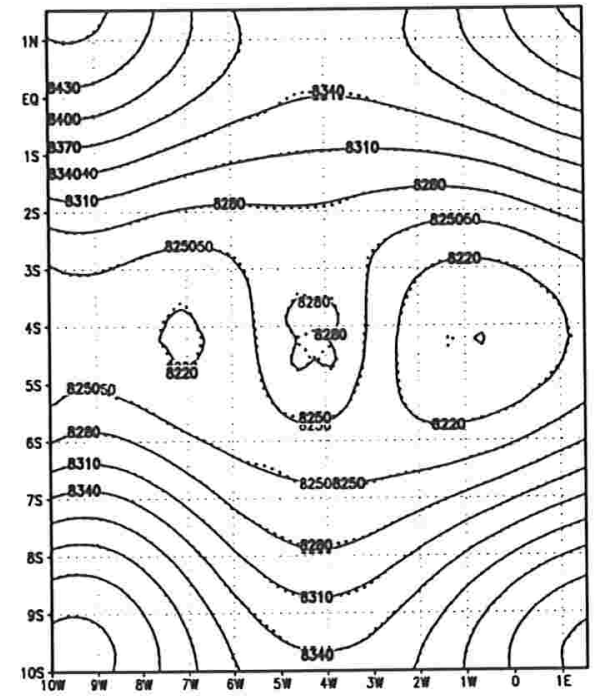

Gros: counces

(c)

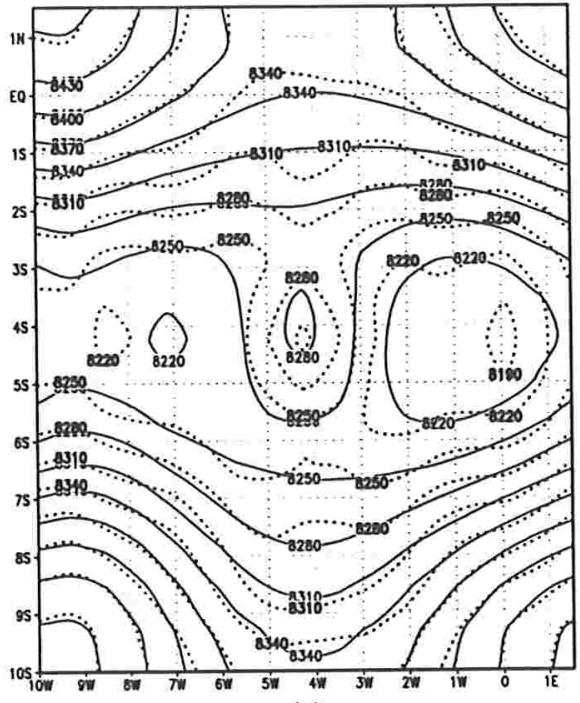

(b)

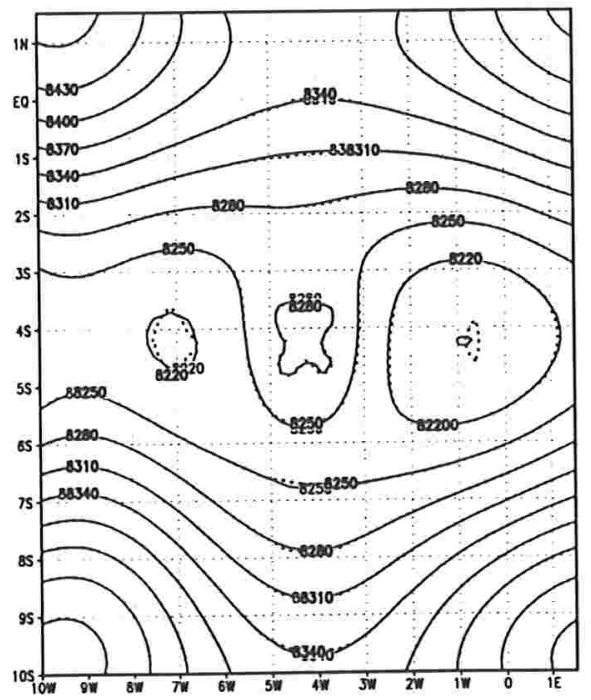

(d)

Figura 2.32: Eq. de água rasa com forçante $F_{1}$ (geopotencial $z+h$ ): GL vs GNL (c/ 90 iter.) (a) $-\mathrm{z}+\mathrm{h}\left(\mathrm{GL}: P_{8}\right) ; \cdots \mathrm{z}+\mathrm{h}\left(\mathrm{Gnl}: P_{8}+Q_{16}\right)$; (b) $-\mathrm{z}+\mathrm{h}\left(\right.$ GL: $\left.P_{16}\right) ; \cdots \mathrm{z}+\mathrm{h}\left(\mathrm{Gnl}: P_{8}+Q_{16}\right)$; $-\mathrm{z}+\mathrm{h}\left(\mathrm{GL}: P_{32}\right) ; \cdots \mathrm{z}+\mathrm{h}\left(\mathrm{Gnl}: P_{16}+Q_{32}\right) ; \quad$ (d) $-\mathrm{z}+\mathrm{h}\left(\mathrm{GL}: P_{64}\right) ; \cdots \mathrm{z}+\mathrm{h}\left(\mathrm{Gnl}: P_{32}+Q_{64}\right) . \quad \Delta t=9$ min. 


\subsection{Análise da Estabilidade}

A análise da estabilidade do método de Galerkin Não Linear para as equações tipo Navier-Stokes em duas dimensões, Burgers em uma dimensão e as equações de KuramotoShivashinsky em duas dimensões tem sido estudada por diversos autores (ver [4], [18], [19], [21], [27], [33], [36], [38], [49], [50], [51], [61], [66], [69]). R. Teman [69] considera a estabilidade das equações de Navier Stokes sem discretização no tempo, enquanto J. Shen [61] faz uma análise da discretização temporal para estas equações. Este ultimo obtém resultados uniformes no tempo, isto é, válidos para intervalos grandes de tempo, usando vários resultados discretos análogos aos Lemas de Gronwall. O principal resultado afirma que a restrição para a estabilidade uniforme em $L^{\infty}\left(\mathbb{R}^{+}, H\right)$ usando a projeção $P_{N / 2}+Q_{d N / 2}$ é do tipo: $\lambda_{N / 2} \Delta t \leq c t e$ (isto é, depende de $\lambda_{N / 2}$ e independe de $\lambda_{j}, j=\frac{N}{2}+1, \cdots, d \frac{N}{2}$ ); enquanto que no método de Galerkin Linear usando a projeção $P_{(d+1) N / 2}$ a restrição da estabilidade é do tipo: $\lambda_{(d+1) N / 2} \Delta t \leq c t e$. Isto é, o método de Galerkin não linear permite passos de tempo maiores que o método de Galerkin linear para um número total equivalente de modos.

Em Agoshkov [2] estuda-se a estabilidade da discretização de primeira ordem no tempo para as equações lagrangianas de água rasa linearizadas em torno de um campo de velocidades $\vec{U}$ constante, sob algumas características do fluxo: difusão dominante $(\vec{U}=0)$, convecção dominante $(\nu=0)$ e profundidade pequena $(H=0)$. Em Elvius [22] tem-se uma análise de estabilidade para discretizações com diferenças finitas no espaço e de segunda ordem no tempo, igualmente linearizadas em torno de um estado constante no espaço e no tempo. Nas discretizações eulerianas das equações de Navier-Stokes é feita uma análise da estabilidade do sistema não linear tanto pelo método de Galerkin Linear como pelo método de Galerkin não linear. O fato de ter-se nas equações de água rasa uma equação adicional, a equação do geopotencial, que substitui a propriedade da divergência nula para as equações de Navier-Stokes, produz complexidade adicional no tratamento dos termos não lineares; isto pode ser explicado porque neste caso o termo $b(\vec{U}, \vec{V}, \vec{V})=\int_{D}(\vec{U} \cdot \nabla \vec{V}) \cdot \vec{V} d x$ não se anula, dificultando a análise da estabilidade não 
linear. Neste trabalho estudamos alguns aspectos da estabilidade para as equações de água rasa com condições de contorno periódicas pelo método de Galerkin linear e não linear (em sua forma diagnóstica). Primeiro linearizamos as equações em torno de um campo de velocidades constantes no espaço e no tempo, obtendo resultados de estabilidade para o método de Galerkin linear com a discretização temporal de segunda ordem estudada nas seções anteriores. A extensão para o caso do método de Galerkin não linear é trivial, pois este se reduz a um método linear neste caso. Mais interessante é a análise da estabilidade do método de Galerkin não linear quando os sistemas dos modos baixos e altos são acoplados, no caso em que outro tipo de linearização é considerada: sob a hipótese de que o campo de velocidades tem uma divergência suficientemente pequena é possível estudar a estabilidade dos esquemas linearizados em torno deste tipo de soluções obtendo condições de estabilidade não muito restritivas. Esta hipótese é justificada pelo fato de que a divergência do campo de velocidades para este tipo de equações se mostra relativamente pequena ( ver por exemplo [14], [30], [53], [56],[57] para o caso das equações barotrópicas, onde o campo de velocidades se comporta como quase não-divergente).

Esta análise é feita para a discretização temporal de primeira ordem, obtendo-se estimativas de energia para o método de Galerkin linear. A extensão para o método de Galerkin não linear é feita, levando a restrições de estabilidade menos severas quando comparando com o método de Galerkin linear com o mesmo número equivalente de modos.

Para a análise da estabilidade usamos a notação para os produtos escalares e normas empregada no capítulo 1 , isto é, $(u, v)=\int_{D} u v d x$ é o produto escalar em $L_{2}$ e $((u, v))=$ $\int_{D} \nabla u \cdot \nabla v d x$ é o produto escalar em $H_{1}$, enquanto $|u|=(u, v)^{1 / 2}$ é a norma em $L_{2} \mathrm{e}$ $\|u\|=((u, v))^{1 / 2}$ indica a norma do gradiente em $H_{1}$. Tambem serão usadas as estimativas D1-D3 e E1-E5 (ver seção 1.1.3) para estimar os produtos escalares que contém os termos linearizados. Por simplicidade e sem perda de generalidade escolhemos $\kappa_{0}=\nu_{0}$. 
2.2.1 Estabilidade: Linearização em torno de estado básico constante (três níveis no tempo)

A discretização das equações de água rasa pelo método de Galerkin Linear com 3 níveis no tempo $\left(t_{n-1}, t_{n}\right.$ e $\left.t_{n+1}\right)$, com os termos lineares dicretizados implicitamente e os não lineares explicitamente no tempo $t_{n}$ leva ao sistema:

$$
\begin{aligned}
& \frac{\vec{V}_{N}^{n+1}-\vec{V}_{N}^{n-1}}{2 \Delta t}+P_{N}\left(\left(\vec{V}_{N}^{n} \cdot \nabla\right) \vec{V}_{N}^{n}\right)+\vec{k} \times \frac{\vec{V}_{N}^{n+1}+\vec{V}_{N}^{n-1}}{2}+\nabla\left(\frac{z_{N}^{n+1}+z_{N}^{n-1}}{2}\right)- \\
& -\nu_{0} \Delta\left(\frac{\vec{V}_{N}^{n+1}+\vec{V}_{N}^{n-1}}{2}\right)=0 \quad \\
& \frac{z_{N}^{n+1}-z_{N}^{n-1}}{2 \Delta t}+g_{0} \nabla \cdot\left(\frac{\vec{V}_{N}^{n+1}+\vec{V}_{N}^{n-1}}{2}\right)+P_{N}\left(\nabla \cdot\left(z_{N}^{n} \vec{V}_{\mathrm{V}}^{n}\right)\right)-\nu_{0} \Delta\left(\frac{z_{N}^{n+1}+z_{N}^{n-1}}{2}\right)=F_{N}
\end{aligned}
$$

A seguir estudamos a estabilidade da discretização (2.89), através da linearização em torno de um campo de velocidades $\vec{U}$ constante. O seguinte esquema resulta da linearização:

\section{ESQUEMA I:}

$$
\begin{array}{r}
\frac{\vec{V}_{N}^{n+1}-\vec{V}_{N}^{n-1}}{2 \Delta t}+\left((\vec{U} \cdot \nabla) \vec{V}_{N}^{n}\right)+\vec{k} \times \frac{\vec{V}_{N}^{n+1}+\vec{V}_{N}^{n-1}}{2}+\nabla\left(\frac{z_{N}^{n+1}+z_{N}^{n-1}}{2}\right)- \\
-\nu_{0} \Delta\left(\frac{\vec{V}_{N}^{n+1}+\vec{V}_{N}^{n-1}}{2}\right)=0 \\
\frac{z_{N}^{n+1}-z_{N}^{n-1}}{2 \Delta t}+g_{0} \nabla \cdot\left(\frac{\vec{V}_{N}^{n+1}+\vec{V}_{N}^{n-1}}{2}\right)+\vec{U} \cdot \nabla z_{N}^{n}-\nu_{0} \Delta\left(\frac{z_{N}^{n+1}+z_{N}^{n-1}}{2}\right)=F_{N}
\end{array}
$$

Para a análise da estabilidade tomamos o produto escalar da primeira equação de (2.90) por $g_{0}\left(\vec{V}_{N}^{n+1}+\vec{V}_{N}^{n-1}\right)$ e da segunda por $\left(z_{N}^{n+1}+z_{N}^{n-1}\right)$. Somando as duas e cancelando os termos provenientes do geopotencial e do divergente devido às condições de contorno periódicas, obtemos:

$$
\begin{array}{r}
g_{0}\left|\vec{V}_{N}^{n+1}\right|^{2}+\left|z_{N}^{n+1}\right|^{2}+g_{0} \nu_{0} \Delta t\left\|\vec{V}_{N}^{n+1}+\vec{V}_{N}^{n-1}\right\|^{2}+\nu_{0} \Delta t\left\|z_{N}^{n+1}+z_{N}^{n-1}\right\|^{2}=g_{0}\left|\vec{V}_{N}^{n-1}\right|^{2}+\left|z_{N}^{n-1}\right|^{2} \\
\quad-2 g_{0} \Delta t\left(\vec{U} \cdot \nabla \vec{V}_{N}^{n}, \vec{V}_{N}^{n+1}+\vec{V}_{N}^{n-1}\right)-2 \Delta t\left(\vec{U} \cdot \nabla z_{N}^{n}, z_{N}^{n+1}+z_{N}^{n-1}\right)+2 \Delta t\left(F_{N}, z_{N}^{n+1}+z_{N}^{n-1}\right)
\end{array}
$$


Devido ao uso de 3 níveis no tempo, somamos o termo $g_{0}\left|\vec{V}_{N}^{n}\right|^{2}+\left|z_{N}^{n}\right|^{2}$ a ambos os lados da última igualdade (assim no processo iterativo as estimativas dependerão das condições iniciais em $t_{0}$ e $\left.t_{1}\right)$ :

$$
\begin{aligned}
G^{n+1}+g_{0} \nu_{0} \Delta t\left\|\vec{V}_{N}^{n+1}+\vec{V}_{N}^{n-1}\right\|^{2}+\nu_{0} \Delta t \| z_{N}^{n+1} & +z_{N}^{n-1} \|^{2}=G^{n}+T N L+ \\
& +2 \Delta t\left(F_{N}, z_{N}^{n+1}+z_{N}^{n-1}\right)
\end{aligned}
$$

onde,

$$
\begin{aligned}
G^{n} & =g_{0}\left|\vec{V}_{N}^{n}\right|^{2}+g_{0}\left|\vec{V}_{N}^{n-1}\right|^{2}+\left|z_{N}^{n}\right|^{2}+\left|z_{N}^{n-1}\right|^{2} \\
T N L & =-2 g_{0} \Delta t\left(\vec{U} \cdot \nabla \vec{V}_{N}^{n}, \vec{V}_{N}^{n+1}+\vec{V}_{N}^{n-1}\right)-2 \Delta t\left(\vec{U} \cdot \nabla z_{N}^{n}, z_{N}^{n+1}+z_{N}^{n-1}\right)
\end{aligned}
$$

O termo que contem a forçante é estimado por:

$$
\begin{aligned}
2 \Delta t\left(F_{N}, z_{N}^{n+1}+z_{N}^{n-1}\right) & \leq 2 \Delta t\left|F_{N}\right|\left|z_{N}^{n+1}+z_{N}^{n-1}\right| \leq 2 \Delta t \lambda_{1}^{-1}\left|F_{N}\right|\left\|z_{N}^{n+1}+z_{N}^{n-1}\right\| \\
& \leq \frac{1}{2} \nu_{0} \Delta t\left\|z_{N}^{n+1}+z_{N}^{n-1}\right\|^{2}+\frac{2}{\nu_{0}} \lambda_{1}^{-2} \Delta t\left|F_{N}\right|^{2}
\end{aligned}
$$

enquanto os termos provenientes da linearização são estimados por:

$$
\begin{aligned}
T N L & =H^{n+1}-H^{n} \\
\text { onde, } \quad H^{n} & =-2 g_{0} \Delta t\left(\vec{U} \cdot \nabla \vec{V}_{N}^{n-1}, \vec{V}_{N}^{n}\right)-2 \Delta t\left(\vec{U} \cdot \nabla z_{N}^{n-1}, z_{N}^{n}\right) .
\end{aligned}
$$

Sustituindo (2.94) e (2.95) em (2.91) obtemos,

$$
\begin{array}{r}
G^{j+1} \leq G^{j+1}+g_{0} \nu_{0} \Delta t\left\|\vec{V}_{N}^{j+1}+\vec{V}_{N}^{j-1}\right\|^{2}+\frac{1}{2} \nu_{0} \Delta t\left\|z_{N}^{j+1}+z_{N}^{j-1}\right\|^{2} \leq G^{j}+H^{j+1}-H^{j}+ \\
+\frac{2}{\nu_{0}} \lambda_{1}^{-2} \Delta t\left|F_{N}\right|^{2}
\end{array}
$$

A soma para $j=1, \cdots, n$ resulta em:

$$
G^{n+1} \leq G^{1}+H^{n+1}-H^{1}+\frac{2}{\nu_{0}} \lambda_{1}^{-2} n \Delta t\left|F_{N}\right|^{2} .
$$

Os termos $H^{j}$ são estimados por:

$$
\left|H^{j}\right| \leq\left(\lambda_{N}^{1 / 2} \Delta t|\vec{U}|_{\infty}\right) G^{j}
$$


Sustituindo (2.99) em (2.98) e tomando o tempo $T>0$ fixo, temos,

$$
\left(1-\lambda_{N}^{1 / 2} \Delta t|\vec{U}|_{\infty}\right) G^{n+1} \leq\left(1+\lambda_{N}^{1 / 2} \Delta t|\vec{U}|_{\infty}\right) G^{1}+\frac{2}{\nu_{0}} \lambda_{1}^{-2} T\left|F_{N}\right|^{2}
$$

Com a condição: $1-\lambda_{N}^{1 / 2} \Delta t|\vec{U}|_{\infty}>0$, ou equivalentemente: $\lambda_{N}^{1 / 2} \Delta t|\vec{U}|_{\infty}<1$, obtemos:

$$
G^{n+1} \leq \frac{1+\lambda_{N}^{1 / 2} \Delta t|\vec{U}|_{\infty}}{1-\lambda_{N}^{1 / 2} \Delta t|\vec{U}|_{\infty}} G^{1}+\frac{2 \nu_{0}^{-1} \lambda_{1}^{-2} T}{1-\lambda_{N}^{1 / 2} \Delta t|\vec{U}|_{\infty}}\left|F_{N}\right|^{2} .
$$

Esta estimativa conduz à seguinte proposição:

Proposição 2.1 Supondo que o passo do tempo $\Delta t$ satisfaça a condição :

$$
\lambda_{N}^{1 / 2} \Delta t|\vec{U}|_{\infty}<1
$$

a estimativa de energia (2.101) é satisfeita, isto é, o Esquema I definido pelo sistema (2.90) é estável para $T>0$ fixo.

A seguir justificamos (2.95) e (2.99) para que a prova da Proposição 2.1 seja completa.

Prova de (2.95): Os termos do produto escalar com super índices $n$ e $n-1$ em (2.93) podem ser expressos como:

$$
\begin{aligned}
-2 g_{0} \Delta t\left(\vec{U} \cdot \nabla \vec{V}_{N}^{n}, \vec{V}_{N}^{n-1}\right) & =-2 g_{0} \Delta t \sum_{i, j=1}^{2} U_{i} \int_{D} \frac{\partial V_{N, j}^{n}}{\partial x_{i}} V_{N, j}^{n-1} d x \\
& =2 g_{0} \Delta t \sum_{i, j=1}^{2} U_{i} \int_{D} \frac{\partial V_{N, j}^{n-1}}{\partial x_{i}} V_{N, j}^{n} d x=2 g_{0} \Delta t\left(\vec{U} \cdot \nabla \vec{V}_{N}^{n-1}, \vec{V}_{N}^{n}\right)
\end{aligned}
$$

Analogamente $-2 \Delta t\left(\vec{U} \cdot \nabla z_{N}^{n}, z_{N}^{n-1}\right)=2 \Delta t\left(\vec{U} \cdot \nabla z_{N}^{n-1}, z_{N}^{n}\right)$. Sustiuindo em (2.93) temos:

$$
\begin{aligned}
T N L= & -2 g_{0} \Delta t\left(\vec{U} \cdot \nabla \vec{V}_{N}^{n}, \vec{V}_{N}^{n+1}\right)-2 \Delta t\left(\vec{U} \cdot \nabla z_{N}^{n}, z_{N}^{n+1}\right)+2 g_{0} \Delta t\left(\vec{U} \cdot \nabla \vec{V}_{N}^{n-1}, \vec{V}_{N}^{n}\right)+ \\
& +2 \Delta t\left(\vec{U} \cdot \nabla z_{N}^{n-1}, z_{N}^{n}\right)=H^{n+1}-H^{n} .
\end{aligned}
$$


Prova de (2.99): De (2.96) temos:

$$
\begin{aligned}
\left|H^{j}\right| & \leq 2 g_{0} \Delta t|\vec{U}|_{\infty}|| \vec{V}_{N}^{j-1}||\left|\vec{V}_{N}^{j}\right|+2 \Delta t|\vec{U}|_{\infty}|| z_{N}^{j-1} \|\left|z_{N}^{j}\right| \\
& \leq 2 g_{0} \lambda_{N}^{1 / 2} \Delta t|\vec{U}|_{\infty}\left|\vec{V}_{N}^{j-1}\right|\left|\vec{V}_{N}^{j}\right|+2 \lambda_{N}^{1 / 2} \Delta t|\vec{U}|_{\infty}\left|z_{N}^{j-1}\right|\left|z_{N}^{j}\right| \\
& \leq \lambda_{N}^{1 / 2} \Delta t|\vec{U}|_{\infty}\left(g_{0}\left|\vec{V}_{N}^{j-1}\right|^{2}+g_{0}\left|\vec{V}_{N}^{j}\right|^{2}+\left|z_{N}^{j-1}\right|^{2}+\left|z_{N}^{j}\right|^{2}\right) \\
& \leq\left(\lambda_{N}^{1 / 2} \Delta t|\vec{U}|_{\infty}\right) G^{j} .
\end{aligned}
$$

Se no Esquema I usamos o método de Galerkin não linear (com a projeção $P_{N / 2}+Q_{N}$ ), obtem-se a condição de estabilidade $\lambda_{N / 2}^{1 / 2} \Delta t|\vec{U}|_{\infty} \leq 1$. A prova é similar à Proposição 2.1, mas com esta linearização ao redor de um campo de velocidade constante no espaço e no tempo se perde totalmente a característica principal deste método que é a interação entre os modos altos e baixos.

\subsubsection{Estabilidade: Linearização em torno de estado básico com dependência espacial ( 2 níveis no tempo)}

As equações de água rasa discretizadas pelo método de Galerkin Linear com 2 níveis no tempo (com os termos lineares dicretizados no tempo $t_{n+1}$ e os termos não lineares no tempo $t_{n}$ ) são expressas pelo sistema:

$$
\begin{aligned}
\frac{\vec{V}_{N}^{n+1}-\vec{V}_{N}^{n}}{\Delta t}+P_{N}\left(\left(\vec{V}_{N}^{n} \cdot \nabla\right) \vec{V}_{N}^{n}\right)+\vec{k} \times \vec{V}_{N}^{n+1}+\nabla z_{N}^{n+1}-\nu_{0} \Delta \vec{V}_{N}^{n+1} & =0 \\
\frac{z_{N}^{n+1}-z_{N}^{n}}{\Delta t}+g_{0} \nabla \cdot \vec{V}_{N}^{n+1}+P_{N}\left(\nabla \cdot\left(z_{N}^{n} \vec{V}_{N}^{n}\right)\right)-\nu_{0} \Delta z_{N}^{n+1} & =F_{N}
\end{aligned}
$$

A seguir estuda-se a Estabilidade do sistema (2.103) linearizado em torno de um campo de velocidades $\vec{U}=\vec{U}(x, y)$ que depende das variáveis espaciais mas não do tempo. Desta linearização temos o seguinte esquema: 
ESQUEMA II:

$$
\begin{aligned}
\frac{\vec{V}_{N}^{n+1}-\vec{V}_{N}^{n}}{\Delta t}+P_{N}\left(\left(\vec{U}_{N} \cdot \nabla\right) \vec{V}_{N}^{n}\right)+\vec{k} \times \vec{V}_{N}^{n+1}+\nabla z_{N}^{n+1}-\nu_{0} \Delta \vec{V}_{N}^{n+1}=0 \\
\frac{z_{N}^{n+1}-z_{N}^{n}}{\Delta t}+g_{0} \nabla \cdot \vec{V}_{N}^{n+1}+P_{N}\left(\nabla \cdot\left(z_{N}^{n} \vec{U}_{N}\right)\right)-\nu_{0} \Delta z_{N}^{n+1}=F_{N}
\end{aligned}
$$

Esta é uma linearização mais interessante que permite interações entre modos altos e baixos para o método de Galerkin não linear. Ainda fazemos uma hipótese adicional no cálculo dos termos linearizados, baseada no fato de que a divergência é costumeiramente pequena em sistemas meterológicos barotrópicos (ver Haltiner [30] e Pedoskli [57]). Na figura 2.18 apresentamos a divergência para vários instantes de tempo que são observados de uma simulação, ilustrando os valores muito pequenos para este campo. Esta hipótese é enunciada como:

Hipótese 2.10 campo de velocidades $\vec{U}_{N}$ sobre o qual foi linearizado o sistema (2.103) pelo sistema (2.104) satisfaz à propriedade:

$$
\left|\nabla \cdot \vec{U}_{N}\right|_{\infty} \leq \epsilon
$$

onde $\epsilon=\frac{1}{4} \nu_{0} \lambda_{1}$ e $\lambda_{1}=2$ é o primeiro autovalor de $-\Delta$ em $[0,2 \pi] \times[0,2 \pi]$.

Para a análise da estabilidade tomamos o produto escalar da primeira equação de (2.104) por $g_{0} \vec{V}_{N}^{n+1}$ e da segunda por $z_{N}^{n+1}$. Tendo em conta a identidade

$$
\left(\vec{V}_{N}^{n+1}-\vec{V}_{N}^{n}, \vec{V}_{N}^{n+1}\right)=\frac{1}{2}\left(\left|\vec{V}_{N}^{n+1}\right|^{2}-\left|\vec{V}_{N}^{n}\right|^{2}+\left|\vec{V}_{N}^{n+1}-\vec{V}_{N}^{n}\right|^{2}\right)
$$

e cancelando os termos $2 g_{0} \Delta t\left(\nabla z_{N}^{n+1}, \vec{V}_{N}^{n+1}\right)+2 g_{0} \Delta t\left(\nabla \cdot \vec{V}_{N}^{n+1}, z_{N}^{n+1}\right)$ devido às condições de contorno periódicas, obtemos ao somar as duas equações anteriores:

$$
\begin{gathered}
g_{0}\left|\vec{V}_{N}^{n+1}\right|^{2}+\left|z_{N}^{n+1}\right|^{2}+g_{0}\left|\vec{V}_{N}^{n+1}-\vec{V}_{N}^{n}\right|^{2}+\left|z_{N}^{n+1}-z_{N}^{n}\right|^{2}+2 g_{0} \nu_{0} \Delta t\left\|\vec{V}_{N}^{n+1}\right\|^{2}+ \\
+2 \nu_{0} \Delta t\left\|z_{N}^{n+1}\right\|^{2}=g_{0}\left|\vec{V}_{N}^{n}\right|^{2}+\left|z_{N}^{n}\right|^{2}+T N L+2 \Delta t\left(F_{N}, z_{N}^{n+1}\right)
\end{gathered}
$$

onde,

$$
T N L=-2 g_{0} \Delta t\left(\vec{U}_{N} \cdot \nabla \vec{V}_{N}^{n}, \vec{V}_{N}^{n+1}\right)-2 \Delta t\left(\nabla \cdot\left(z_{N}^{n} \vec{U}_{N}\right), z_{N}^{n+1}\right)
$$


Usando a Hipótese (2.1) e supondo $c_{1}^{2} \lambda_{N}^{1 / 2} \Delta t\left|\vec{U}_{N}\right|\left\|\vec{U}_{N}\right\| \leq \frac{3}{8} \nu_{0}$, obtemos a estimativa:

$$
\begin{aligned}
|T N L|+2 \Delta t\left|\left(F_{N}, z_{N}^{n+1}\right)\right| & \leq g_{0}\left|\vec{V}_{N}^{n+1}-\vec{V}_{N}^{n}\right|^{2}+\left|z_{N}^{n+1}-z_{N}^{n}\right|^{2}+\nu_{0} \Delta t\left\|z_{N}^{n+1}\right\|^{2}+ \\
& +g_{0} \nu_{0} \Delta t\left\|\vec{V}_{N}^{n}\right\|^{2}+\nu_{0} \Delta t\left\|z_{N}^{n}\right\|^{2}+2 \nu_{0}^{-1} \lambda_{1}^{2} \Delta t\left|F_{N}\right|^{2}
\end{aligned}
$$

Substituindo (2.108) em (2.106) (trocando para $j$ o índice superior) temos a inequação:

$$
\begin{array}{r}
g_{0}\left|\vec{V}_{N}^{j+1}\right|^{2}+\left|z_{N}^{j+1}\right|^{2}+2 g_{0} \nu_{0} \Delta t\left\|\vec{V}_{N}^{j+1}\right\|^{2}+\nu_{0} \Delta t\left\|z_{N}^{j+1}\right\|^{2} \leq g_{0}\left|\vec{V}_{N}^{j}\right|^{2}+\left|z_{N}^{j}\right|^{2}+ \\
+g_{0} \nu_{0} \Delta t\left\|\vec{V}_{N}^{j}\right\|^{2}+\nu_{0} \Delta t\left\|z_{N}^{j}\right\|^{2}+2 \nu_{0}^{-1} \lambda_{1}^{2} \Delta t\left|F_{N}\right|^{2}
\end{array}
$$

A soma dos termos $j=0, \cdots, n$ nos dá a estimativa de energia:

$$
\begin{gathered}
g_{0}\left|\vec{V}_{N}^{n+1}\right|^{2}+\left|z_{N}^{n+1}\right|^{2}+2 g_{0} \nu_{0} \Delta t\left\|\vec{V}_{N}^{n+1}\right\|^{2}+g_{0} \nu_{0} \Delta t \sum_{j=1}^{n}\left\|\vec{V}_{N}^{j}\right\|^{2}+\nu_{0} \Delta t\left\|z_{N}^{n+1}\right\|^{2} \\
\leq g_{0}\left|\vec{V}_{N}^{0}\right|^{2}++\left|z_{N}^{0}\right|^{2}+g_{0} \nu_{0} \Delta t\left\|\vec{V}_{N}^{0}\right\|^{2}+\nu_{0} \Delta t\left\|z_{N}^{0}\right\|^{2}+2 \nu_{0}^{-1} \lambda_{1}^{2} T\left|F_{N}\right|^{2}
\end{gathered}
$$

onde $n \Delta t \leq T$, para $T>0$ fixo.

Esta estimativa leva à seguinte proposição:

Proposição 2.2 Sejam os dados iniciais $\vec{V}_{N}^{0}$, $z_{N}^{0}$, e $\vec{U}_{N}$ no espaço $\mathbb{H}_{\text {per }}^{1}$. Se a Hipótese (2.1) é verificada, e o passo do tempo $\Delta t$ satisfaz a condição:

$$
c_{1}^{2} \lambda_{N}^{1 / 2} \Delta t\left|\vec{U}_{N}\right|\left\|\vec{U}_{N}\right\| \leq \frac{3}{8} \nu_{0}
$$

então a estimativa de energia (2.109) é satisfeita, isto é, o Esquema II definido pelo sistema (2.104) é estável para $T>0$ fixo.

A seguir a estimativa (2.108) é detalhada, completando a prova da Proposição 2.2.

Prova de (2.108): Usando as estimativas D1 e E1, avaliamos os termos linearizados em (2.107) :

a1) $-2 g_{0} \Delta t\left(\vec{U}_{N} \cdot \nabla \vec{V}_{N}^{n}, \vec{V}_{N}^{n+1}\right)=-2 g_{0} \Delta t\left(\vec{U}_{N} \cdot \nabla \vec{V}_{N}^{n}, \vec{V}_{N}^{n+1}-\vec{V}_{N}^{n}\right)-2 g_{0} \Delta t\left(\vec{U}_{N} \cdot \nabla \vec{V}_{N}^{n}, \vec{V}_{N}^{n}\right)$ e: 
$-2 g_{0} \Delta t\left(\vec{U}_{N} \cdot \nabla \vec{V}_{N}^{n}, \vec{V}_{N}^{n+1}-\vec{V}_{N}^{n}\right) \leq 2 c_{1} g_{0} \Delta t\left|\vec{U}_{N}\right|^{\frac{1}{2}}\left\|\vec{U}_{N}\right\|^{\frac{1}{2}}\left\|\vec{V}_{N}^{n}\right\|\left|\vec{V}_{N}^{n+1}-\vec{V}_{N}^{n}\right|^{\frac{1}{2}}\left\|\vec{V}_{N}^{n+1}-\vec{V}_{N}^{n}\right\|^{\frac{1}{2}}$

$$
\begin{aligned}
& \leq 2 c_{1} g_{0} \lambda_{N}^{1 / 4} \Delta t\left|\vec{U}_{N}\right|^{1 / 2}\left\|\vec{U}_{N}\right\|^{1 / 2}\left\|\vec{V}_{N}^{n}\right\|\left|\vec{V}_{N}^{n+1}-\vec{V}_{N}^{n}\right| \\
& \leq g_{0}\left|\vec{V}_{N}^{n+1}-\vec{V}_{N}^{n}\right|^{2}+c_{1}^{2} \Delta^{2} t g_{0} \lambda_{N}^{1 / 2}\left|\vec{U}_{N}\right|\left\|\vec{U}_{N}\right\|\left\|\vec{V}_{N}^{n}\right\|^{2},
\end{aligned}
$$

$$
\begin{aligned}
-2 g_{0} \Delta t\left(\vec{U}_{N} \cdot \nabla \vec{V}_{N}^{n}, \vec{V}_{N}^{n}\right) & =g_{0} \Delta t \int_{D}\left(\nabla \cdot \vec{U}_{N}\right)\left(\vec{V}_{N}^{n} \cdot \vec{V}_{N}^{n}\right) \leq g_{0} \Delta t\left|\nabla \cdot \vec{U}_{N}\right|_{\infty} \int_{D}\left(\vec{V}_{N}^{n} \cdot \vec{V}_{N}^{n}\right) \\
& \leq g_{0} \Delta t\left|\nabla \cdot \vec{U}_{N}\right|_{\infty}\left|\vec{V}_{N}^{n}\right|^{2} \leq g_{0} \lambda_{1}^{-1} \Delta t\left|\nabla \cdot \vec{U}_{N}\right|_{\infty}\left\|\vec{V}_{N}^{n}\right\|^{2}
\end{aligned}
$$

a2) $-2 \Delta t\left(\nabla \cdot\left(z_{N}^{n} \vec{U}_{N}\right), z_{N}^{n+1}\right)=-2 \Delta t\left(z_{N}^{n} \nabla \cdot \vec{U}_{N}, z_{N}^{n+1}\right)-2 \Delta t\left(\vec{U}_{N} \cdot \nabla z_{N}^{n}, z_{N}^{n+1}\right)$.

Ainda:

$-2 \Delta t\left(z_{N}^{n} \nabla \cdot \vec{U}_{N}, z_{N}^{n+1}\right) \leq 2 \Delta t\left|\nabla \cdot \vec{U}_{N}\right|_{\infty}\left|z_{N}^{n}\right|\left|z_{N}^{n+1}\right| \leq 2 \Delta t \lambda_{1}^{-1}\left|\nabla \cdot \vec{U}_{N}\right|_{\infty}\left\|z_{N}^{n}\right\|\left\|z_{N}^{n+1}\right\|$

$$
\leq \frac{1}{2} \nu_{0} \Delta t\left\|z_{N}^{n+1}\right\|^{2}+2 \nu_{0}^{-1} \lambda_{1}^{-2} \Delta t\left|\nabla \cdot \vec{U}_{N}\right|_{\infty}^{2}\left\|z_{N}^{n}\right\|^{2}
$$

$$
\begin{aligned}
& -2 \Delta t\left(\vec{U}_{N} \cdot \nabla z_{N}^{n}, z_{N}^{n+1}\right)=-2 \Delta t\left(\vec{U}_{N} \cdot \nabla z_{N}^{n}, z_{N}^{n+1}-z_{N}^{n}\right)-2 \Delta t\left(\vec{U}_{N} \cdot \nabla z_{N}^{n}, z_{N}^{n}\right) \\
& \quad \leq 2 \Delta t c_{1} \lambda_{N}^{1 / 4}\left|\vec{U}_{N}\right|^{1 / 2}\left\|\vec{U}_{N}\right\|^{1 / 2}\left\|z_{N}^{n}\right\|\left|z_{N}^{n+1}-z_{N}^{n}\right|+\Delta t \int_{D}\left(\nabla \cdot \vec{U}_{N}\right)\left(z_{N}^{n}\right)^{2} \\
& \leq\left|z_{N}^{n+1}-z_{N}^{n}\right|^{2}+c_{1}^{2} \lambda_{N}^{1 / 2} \Delta^{2} t\left|\vec{U}_{N}\right|\left\|\vec{U}_{N}\right\|\left\|z_{N}^{n}\right\|^{2}+\lambda_{1}^{-1} \Delta t\left|\nabla \cdot \vec{U}_{N}\right|_{\infty}\left\|z_{N}^{n}\right\|^{2}
\end{aligned}
$$

a3) $2 \Delta t\left(F_{N}, z_{N}^{n+1}\right) \leq 2 \Delta t \lambda_{1}^{-1}\left|F_{N}\right|\left\|z_{N}^{n+1}\right\| \leq \frac{1}{2} \nu_{0} \Delta t\left\|z_{N}^{n+1}\right\|^{2}+2 \nu_{0}^{-1} \lambda_{1}^{-2} \Delta t\left|F_{N}\right|^{2}$

Sob a Hipótese (2.1) e a condição (2.110) os termos contendo produtos são simplificados por:

a4) $c_{1}^{2} \Delta^{2} t g_{0} \lambda_{N}^{1 / 2}\left|\vec{U}_{N}\right|\left\|\vec{U}_{N}\right\|\left\|\vec{V}_{N}^{n}\right\|^{2} \leq\left(c_{1}^{2} \Delta t \lambda_{N}^{1 / 2}\left|\vec{U}_{N}\right|\left\|\vec{U}_{N}\right\|\right) g_{0} \Delta t\left\|\vec{V}_{N}^{n}\right\|^{2}$

$$
\leq \frac{3}{8} g_{0} \nu_{0} \Delta t\left\|\vec{V}_{N}^{n}\right\|^{2}
$$

$g_{0} \lambda_{1}^{-1} \Delta t\left|\nabla \cdot \vec{U}_{N}\right|_{\infty}\left\|\vec{V}_{N}^{n}\right\|^{2} \leq g_{0} \lambda_{1}^{-1} \Delta t\left(\frac{1}{4} \nu_{0} \lambda_{1}\right)\left\|\vec{V}_{N}^{n}\right\|^{2} \leq \frac{1}{4} g_{0} \nu_{0} \Delta t\left\|\vec{V}_{N}^{n}\right\|^{2}$

a5) $2 \nu_{0}^{-1} \lambda_{1}^{-2} \Delta t\left|\nabla \cdot \vec{U}_{N}\right|_{\infty}^{2}\left\|z_{N}^{n}\right\|^{2} \leq 2 \nu_{0}^{-1} \lambda_{1}^{-2} \Delta t\left(\frac{1}{16} \nu_{0}^{2} \lambda_{1}^{2}\right)\left\|z_{N}^{n}\right\|^{2} \leq \frac{1}{8} \nu_{0} \Delta t\left\|z_{N}^{n}\right\|^{2}$

$c_{1}^{2} \lambda_{N}^{1 / 2} \Delta^{2} t\left|\vec{U}_{N}\right|\left\|\vec{U}_{N}\right\|\left\|z_{N}^{n}\right\|^{2} \leq\left(c_{1}^{2} \lambda_{N}^{1 / 2} \Delta t\left|\vec{U}_{N}\right|\left\|\vec{U}_{N}\right\|\right) \Delta t\left\|z_{N}^{n}\right\|^{2} \leq \frac{3}{8} \cdot \nu_{0} \Delta t\left\|z_{N}^{n}\right\|^{2}$ $\lambda_{1}^{-1} \Delta t\left|\nabla \cdot \vec{U}_{N}\right|_{\infty}\left\|z_{N}^{n}\right\|^{2} \leq \lambda_{1}^{-1} \Delta t\left(\frac{1}{4} \nu_{0} \lambda_{1}\right)\left\|z_{N}^{n}\right\|^{2} \leq \frac{1}{4} \nu_{0} \Delta t\left\|z_{N}^{n}\right\|^{2}$

Combinando a1-a5 a estimativa (2.108) é verificada.

A restrição (2.110) depende da forma de estimar o termo TNL em (2.107); assumimos 
aqui os dados iniciais e a velocidade caracteristica $\vec{V}_{N}$ em $\mathbb{H}_{p e r}^{1}$. Se admitirmos que estejam em $\mathbb{L}_{p e r}^{2}$, a restrição da estabilidade é mais forte: $c_{1}^{2} \lambda_{N} \Delta t\left|\vec{U}_{N}\right|^{2} \leq$ cte $\nu_{0}$. Ainda, se em lugar de usar a estimativa $\mathbf{E} 1$, os termos em $T N L$ forem avaliados usando a norma $|\cdot|_{\infty}$ para a velocidade caracteristica $\vec{U}_{N}$ a restrição de estabilidade é do tipo: $\Delta t|\vec{U}|_{\infty} \leq$ cte $\nu_{0}$, onde não se tem relação entre $\Delta t$ e $\lambda_{N}$.

As equações do método de Galerkin não linear com os modos altos avaliados em forma diagnóstica (associada ao sistema (2.103)) é descrita pelo sistema:

$$
\begin{aligned}
\frac{\vec{V}_{p}^{n+1}-\vec{V}_{p}^{n}}{\Delta t}+\vec{k} \times \vec{V}_{p}^{n+1}+\nabla z_{p}^{n+1}-\nu_{0} \Delta \vec{V}_{p}^{n+1}= & -P_{N / 2}\left(\left(\vec{V}_{p}^{n} \cdot \nabla\right) \vec{V}_{p}^{n}+\right. \\
& \left.+\left(\vec{V}_{q}^{n+1} \cdot \nabla\right) \vec{V}_{p}^{n}+\left(\vec{V}_{p}^{n} \cdot \nabla\right) \vec{V}_{q}^{n+1}\right) \\
\frac{z_{p}^{n+1}-z_{p}^{n}}{\Delta t}+g_{0} \nabla \cdot \vec{V}_{p}^{n+1}-\nu_{0} \Delta z_{p}^{n+1}= & P_{N / 2} F-P_{N / 2}\left(\nabla \cdot\left(z_{p}^{n} \vec{V}_{p}^{n}\right)+\right. \\
& \left.+\nabla \cdot\left(z_{q}^{n+1} \vec{V}_{p}^{n}\right)+\nabla \cdot\left(z_{p}^{n} \vec{V}_{q}^{n+1}\right)\right) \\
\vec{k} \times \vec{V}_{q}^{n+1}+\nabla z_{q}^{n+1}-\nu_{0} \Delta \vec{V}_{q}^{n+1}= & -Q_{N}\left(\left(\vec{V}_{p}^{n} \cdot \nabla\right) \vec{V}_{p}^{n}\right) \\
g_{0} \nabla \cdot \vec{V}_{q}^{n+1}-\nu_{0} \Delta z_{q}^{n+1} & =Q_{N} F-Q_{N}\left(\nabla \cdot\left(z_{p}^{n} \vec{V}_{p}^{n}\right)\right)
\end{aligned}
$$

onde:

$$
\vec{V}_{p}^{n}=P_{N / 2}\left(\vec{V}^{n}\right), \vec{V}_{q}^{n}=Q_{N}\left(\vec{V}^{n}\right), z_{p}^{n}=P_{N / 2}\left(z^{n}\right), z_{q}^{n}=Q_{N}\left(z^{n}\right), \text { e } Q_{N}=P_{N}-P_{N / 2} .
$$

A seguir considera-se o análogo ao Esquema II: o sistema (2.111) é linearizado em torno de um campo de velocidades $\vec{U}_{N}=\vec{U}_{N}(x, y)$ dependendo das componentes espaciais, mas independente do tempo: 
ESQUEMA IIa:

$$
\begin{aligned}
\frac{\vec{V}_{p}^{n+1}-\vec{V}_{p}^{n}}{\Delta t}+\vec{k} \times \vec{V}_{p}^{n+1}+\nabla z_{p}^{n+1}-\nu_{0} \Delta \vec{V}_{p}^{n+1} & =-P_{N / 2}\left(\left(\vec{U}_{p} \cdot \nabla\right) \vec{V}_{p}^{n}+\right. \\
& \left.+\left(\vec{U}_{q} \cdot \nabla\right) \vec{V}_{p}^{n}+\left(\vec{U}_{p} \cdot \nabla\right) \vec{V}_{q}^{n+1}\right) \\
\frac{z_{p}^{n+1}-z_{p}^{n}}{\Delta t}+g_{0} \nabla \cdot \vec{V}_{p}^{n+1}-\nu_{0} \Delta z_{p}^{n+1} & =P_{N / 2} F-P_{N / 2}\left(\nabla \cdot\left(z_{p}^{n} \vec{U}_{p}\right)+\right. \\
& \left.+\nabla \cdot\left(z_{q}^{n+1} \vec{U}_{p}\right)+\nabla \cdot\left(z_{p}^{n} \vec{U}_{q}\right)\right) \\
\vec{k} \times \vec{V}_{q}^{n+1}+\nabla z_{q}^{n+1}-\nu_{0} \Delta \vec{V}_{q}^{n+1} & =-Q_{N}\left(\left(\vec{U}_{p} \cdot \nabla\right) \vec{V}_{p}^{n}\right) \\
g_{0} \nabla \cdot \vec{V}_{q}-\nu_{0} \Delta z_{q}^{n+1} & =Q_{N} F-Q_{N}\left(\nabla \cdot\left(z_{p}^{n} \vec{U}_{p}\right)\right)
\end{aligned}
$$

onde, $\vec{U}_{p}=P_{N / 2}(\vec{U})$ e $\vec{U}_{q}=Q_{N}(\vec{U})$.

Tomando o produto escalar da primeira equação de (2.112) com $g_{0} \vec{V}_{p}^{n+1}$, da segunda com $z_{p}^{n+1}$, da terceira com $2 g_{0} \Delta t \vec{V}_{q}^{n+1}$, e da quarta com $2 g_{0} \Delta t z_{q}^{n+1}$; e somando, obtemos:

$$
\begin{aligned}
g_{0}\left|\vec{V}_{p}^{n+1}\right|^{2} & +\left|z_{p}^{n+1}\right|^{2}+g_{0}\left|\vec{V}_{p}^{n+1}-\vec{V}_{p}^{n}\right|^{2}+\left|z_{p}^{n+1}-z_{p}^{n}\right|^{2}+2 \nu_{0} g_{0} \Delta t\left\|\vec{V}_{p}^{n+1}\right\|^{2}+ \\
& +2 \nu_{0} \Delta t\left\|z_{p}^{n+1}\right\|^{2}+2 \nu_{0} g_{0} \Delta t\left\|\vec{V}_{q}^{n+1}\right\|^{2}+2 \nu_{0} \Delta t\left\|z_{q}^{n+1}\right\|^{2} \\
= & g_{0}\left|\vec{V}_{p}^{n}\right|^{2}+\left|z_{p}^{n}\right|^{2}+2 \Delta t\left(P_{N / 2} F, z_{p}^{n+1}\right)+2 \Delta t\left(Q_{N} F, z_{p}^{n+1}\right)+G(p, q)
\end{aligned}
$$

onde,

$$
\begin{aligned}
G= & -2 g_{0} \Delta t\left(\vec{U}_{p} \cdot \nabla \vec{V}_{p}^{n}, \vec{V}_{p}^{n+1}\right)-2 g_{0} \Delta t\left(\vec{U}_{q} \cdot \nabla \vec{V}_{p}^{n}, \vec{V}_{p}^{n+1}\right)-2 g_{0} \Delta t\left(\vec{U}_{p} \cdot \nabla \vec{V}_{q}^{n+1}, \vec{V}_{p}^{n+1}\right)- \\
& -2 \Delta t\left(\nabla \cdot\left(z_{p}^{n} \vec{U}_{p}\right), z_{p}^{n+1}\right)-2 \Delta t\left(\nabla \cdot\left(z_{q}^{n+1} \vec{U}_{p}\right), z_{p}^{n+1}\right)-2 \Delta t\left(\nabla \cdot\left(z_{p}^{n} \vec{U}_{q}\right), z_{p}^{n+1}\right)- \\
& -2 g_{0} \Delta t\left(\vec{U}_{p} \cdot \nabla \vec{V}_{p}^{n}, \vec{V}_{q}^{n+1}\right)-2 \Delta t\left(\nabla \cdot\left(z_{p}^{n} \vec{U}_{p}\right), z_{q}^{n+1}\right)
\end{aligned}
$$

Agora, sob a Hipotese $(2.1)$ e a condição: $\quad c_{1}^{2} \lambda_{N / 2}^{1 / 2} \Delta t\left(\left|\vec{U}_{p}\right|\left\|\vec{U}_{p}\right\|+\left|\vec{U}_{q}\right|\left\|\vec{U}_{q}\right\|\right) \leq \frac{1}{8} \nu_{0}$, a seguinte estimativa é obtida:

$$
\begin{aligned}
G+2 \Delta t\left(P_{N / 2} F, z_{p}^{n+1}\right)+2 \Delta t( & \left.Q_{N} F, z_{p}^{n+1}\right) \leq g_{0}\left|\vec{V}_{p}^{n+1}-\vec{V}_{p}^{n}\right|^{2}+\left|z_{p}^{n+1}-z_{p}^{n}\right|^{2}+ \\
& +\nu_{0} \Delta t\left\|z_{p}^{n+1}\right\|^{2}+2 \nu_{0} g_{0} \Delta t\left\|\vec{V}_{q}^{n+1}\right\|^{2}+2 \nu_{0} \Delta t\left\|z_{q}^{n+1}\right\|^{2}+
\end{aligned}
$$




$$
\begin{aligned}
& +\nu_{0} g_{0} \Delta t\left\|\vec{V}_{p}^{n}\right\|^{2}+\nu_{0} \Delta t\left\|z_{p}^{n}\right\|^{2}+4 \nu_{0}^{-1} \lambda_{1}^{-1} \Delta t\left|P_{N / 2} F\right|^{2}+ \\
& +\nu_{0}^{-1} \lambda_{N / 2}^{-1} \Delta t\left|Q_{N} F\right|^{2}
\end{aligned}
$$

Sustituindo (2.115) em (2.113) temos (após trocar o indice superior para $j$ ):

$$
\begin{array}{r}
g_{0}\left|\vec{V}_{p}^{j+1}\right|^{2}+\left|z_{p}^{j+1}\right|^{2}+2 \nu_{0} g_{0} \Delta t\left\|\vec{V}_{p}^{j+1}\right\|^{2}+\nu_{0} \Delta t\left\|z_{p}^{j+1}\right\|^{2} \leq g_{0}\left|\vec{V}_{p}^{j}\right|^{2}+\left|z_{p}^{j}\right|^{2}+g_{0} \nu_{0} \Delta t\left\|\vec{V}_{p}^{j}\right\|^{2}+ \\
+\nu_{0} \Delta t\left\|z_{p}^{j}\right\|^{2}+\frac{4 \Delta t}{\nu_{0} \lambda_{1}}\left|P_{N / 2} F\right|^{2}+\frac{\Delta t}{\nu_{0} \lambda_{N / 2}}\left|Q_{N} F\right|^{2}
\end{array}
$$

Somando $j=0, \cdots, n$ na desigualdade anterior e cancelando termos obtemos a estimativa de energia:

$$
\begin{aligned}
& g_{0}\left|\vec{V}_{p}^{n+1}\right|^{2}+\left|z_{p}^{n+1}\right|^{2}+2 \nu_{0} g_{0} \Delta t\left\|\vec{V}_{p}^{n+1}\right\|^{2}+\nu_{0} g_{0} \Delta t \sum_{j=1}^{n}\left\|\vec{V}_{p}^{j+1}\right\|^{2}+\nu_{0} \Delta t\left\|z_{p}^{n+1}\right\|^{2} \\
& \leq g_{0}\left|\vec{V}_{p}^{0}\right|^{2}+\left|z_{p}^{0}\right|^{2}+g_{0} \nu_{0} \Delta t\left\|\vec{V}_{p}^{0}\right\|^{2}+\nu_{0} \Delta t\left\|z_{p}^{0}\right\|^{2}+\frac{4 T}{\nu_{0} \lambda_{1}}\left|P_{N / 2} F\right|^{2}+\frac{T}{\nu_{0} \lambda_{N / 2}} \mid Q_{N} F(2.116)
\end{aligned}
$$

onde $n \Delta t \leq T$, para $T>0$ fixo.

Esta estimativa leva à seguinte proposição:

Proposição 2.3 Sejam os dados iniciais $\vec{V}_{p}^{0}, \vec{V}_{q}^{0}, z_{p}^{0}$, $z_{q}^{0}$, e as velocidades características $\vec{U}_{p}, \vec{U}_{q}$ em $\mathbb{H}_{p e r}^{1}$. Se a Hipótese (2.1) é verificada, e o passo do tempo $\Delta t$ satisfaz à condição:

$$
c_{1}^{2} \lambda_{N / 2}^{1 / 2} \Delta t\left(\left|\vec{U}_{p}\right|\left\|\vec{U}_{p}\right\|+\left|\vec{U}_{q}\right|\left\|\vec{U}_{q}\right\|\right) \leq \frac{1}{8} \nu_{0}
$$

então a estimativa de energia (2.116) é satisfeita, isto é, o Esquema IIa definido pelo sistema (2.112) é estável para $T>0$ fixo.

A seguir justificamos a estimativa (2.115) para que a prova da Proposição 2.3 seja completa.

Prova de (2.115): O termo $G$ em (2.114) pode-se decompor da seguinte forma:

$$
G=-2 g_{0} \Delta t\left(\vec{U}_{p} \cdot \nabla \vec{V}_{p}^{n}, \vec{V}_{p}^{n+1}-\vec{V}_{p}^{n}\right)-2 g_{0} \Delta t\left(\vec{U}_{q} \cdot \nabla \vec{V}_{p}^{n}, \vec{V}_{p}^{n+1}-\vec{V}_{p}^{n}\right)-
$$




$$
\begin{aligned}
& -2 g_{0} \Delta t\left(\vec{U}_{p} \cdot \nabla \vec{V}_{q}^{n+1}, \vec{V}_{p}^{n+1}-\vec{V}_{p}^{n}\right)-2 g_{0} \Delta t\left(\vec{U}_{p} \cdot \nabla \vec{V}_{q}^{n+1}, \vec{V}_{p}^{n}\right)- \\
& -2 g_{0} \Delta t\left(\vec{U}_{p} \cdot \nabla \vec{V}_{p}^{n}, \vec{V}_{q}^{n+1}\right)-2 \Delta t\left(z_{p}^{n} \nabla \cdot \vec{U}_{p}, z_{p}^{n+1}\right)-2 \Delta t\left(z_{p}^{n} \nabla \cdot \vec{U}_{q}, z_{p}^{n+1}\right)- \\
& -2 \Delta t\left(\vec{U}_{p} \cdot \nabla z_{q}^{n+1}, z_{p}^{n+1}-z_{p}^{n}\right)-\Delta t\left(z_{p}^{n} \nabla \cdot \vec{U}_{p}, z_{p}^{n}\right)-\Delta t\left(z_{p}^{n} \nabla \cdot \vec{U}_{q}, z_{p}^{n}\right)- \\
& -2 \Delta t\left(\vec{U}_{p} \cdot \nabla z_{p}^{n}, z_{p}^{n+1}-z_{p}^{n}\right)-2 \Delta t\left(\vec{U}_{q} \cdot \nabla z_{p}^{n}, z_{p}^{n+1}-z_{p}^{n}\right)- \\
& -2 \Delta t\left(z_{q}^{n+1} \nabla \cdot \vec{U}_{p}, z_{p}^{n+1}\right)-2 g_{0} \Delta t\left(\vec{U}_{p} \cdot \nabla \vec{V}_{p}^{n}, \vec{V}_{p}^{n}\right)-2 g_{0} \Delta t\left(\vec{U}_{q} \cdot \nabla \vec{V}_{p}^{n}, \vec{V}_{p}^{n}\right)
\end{aligned}
$$

estes termos são estimados como segue:

b1) $-2 g_{0} \Delta t\left(\vec{U}_{p} \cdot \nabla \vec{V}_{p}^{n}, \vec{V}_{p}^{n+1}-\vec{V}_{p}^{n}\right) \leq 2 g_{0} \Delta t c_{1} \lambda_{N / 2}^{1 / 4}\left|\vec{U}_{p}\right|^{1 / 2}\left\|\vec{U}_{p}\right\|^{1 / 2}\left\|\vec{V}_{p}^{n}\right\|\left|\vec{V}_{p}^{n+1}-\vec{V}_{p}^{n}\right|$

$$
\leq \frac{1}{2} \epsilon_{1} g_{0}\left|\vec{V}_{p}^{n+1}-\vec{V}_{p}^{n}\right|^{2}+2 \epsilon_{1}^{-1} g_{0} \Delta^{2} t c_{1}^{2} \lambda_{N / 2}^{1 / 2}\left|\vec{U}_{p}\right|\left\|\vec{U}_{p}\right\|\left\|\vec{V}_{p}^{n}\right\|^{2}
$$

b2) $-2 g_{0} \Delta t\left(\vec{U}_{q} \cdot \nabla \vec{V}_{p}^{n}, \vec{V}_{p}^{n+1}-\vec{V}_{p}^{n}\right) \leq 2 g_{0} \Delta t c_{1} \lambda_{N / 2}^{1 / 4}\left|\vec{U}_{q}\right|^{1 / 2}\left\|\vec{U}_{q}\right\|^{1 / 2}\left\|\vec{V}_{p}^{n}\right\|\left|\vec{V}_{p}^{n+1}-\vec{V}_{p}^{n}\right|$

$$
\leq \frac{1}{2} \epsilon_{1} g_{0}\left|\vec{V}_{p}^{n+1}-\vec{V}_{p}^{n}\right|^{2}+2 \epsilon_{1}^{-1} g_{0} \Delta^{2} t c_{1}^{2} \lambda_{N / 2}^{1 / 2}\left|\vec{U}_{q}\right|\left\|\vec{U}_{q}\right\|\left\|\vec{V}_{p}^{n}\right\|^{2}
$$

b3) $-2 g_{0} \Delta t\left(\vec{U}_{p} \cdot \nabla \vec{V}_{q}^{n+1}, \vec{V}_{p}^{n+1}-\vec{V}_{p}^{n}\right) \leq 2 g_{0} \Delta t c_{1} \lambda_{N / 2}^{1 / 4}\left|\vec{U}_{p}\right|^{1 / 2}\left\|\vec{U}_{p}\right\|^{1 / 2}\left\|\vec{V}_{q}^{n+1}\right\|\left|\vec{V}_{p}^{n+1}-\vec{V}_{p}^{n}\right|$

$$
\leq \frac{1}{2} \epsilon_{1} g_{0}\left|\vec{V}_{p}^{n+1}-\vec{V}_{p}^{n}\right|^{2}+2 \epsilon_{1}^{-1} g_{0} \Delta^{2} t c_{1}^{2} \lambda_{N / 2}^{1 / 2}\left|\vec{U}_{p}\right|\left\|\vec{U}_{p}\right\|\left\|\vec{V}_{q}^{n+1}\right\|^{2}
$$

b4) $-2 g_{0} \Delta t\left(\vec{U}_{p} \cdot \nabla \vec{V}_{q}^{n+1}, \vec{V}_{p}^{n}\right)-2 g_{0} \Delta t\left(\vec{U}_{p} \cdot \nabla \vec{V}_{p}^{n}, \vec{V}_{q}^{n+1}\right)=2 g_{0} \Delta t \int_{D}\left(\nabla \cdot \vec{U}_{p}\right)\left(\vec{V}_{q}^{n+1} \cdot \vec{V}_{p}^{n}\right)$ $\leq 2 g_{0} \Delta t\left|\nabla \cdot \vec{U}_{p}\right|_{\infty}\left|\vec{V}_{q}^{n+1}\right|\left|\vec{V}_{p}^{n}\right| \leq 2 g_{0} \Delta t \lambda_{1}^{-1 / 2} \lambda_{N / 2}^{-1 / 2}\left|\nabla \cdot \vec{U}_{p}\right|_{\infty}\left\|\vec{V}_{q}^{n+1}\right\|\left\|\vec{V}_{p}^{n}\right\|$ $\leq \frac{1}{2} \epsilon_{3} g_{0} \nu_{0} \Delta t\left\|\vec{V}_{q}^{n+1}\right\|^{2}+2 \epsilon_{3}^{-1} g_{0} \nu_{0}^{-1} \lambda_{1}^{-1} \lambda_{N / 2}^{-1} \Delta t\left|\nabla \cdot \vec{U}_{p}\right|_{\infty}^{2}\left\|\vec{V}_{p}^{n}\right\|^{2}$

b5) $-2 \Delta t\left(z_{p}^{n} \nabla \cdot \vec{U}_{p}, z_{p}^{n+1}\right) \leq 2 \Delta t\left|\nabla \cdot \vec{U}_{p}\right|_{\infty}\left|z_{p}^{n}\right|\left|z_{p}^{n+1}\right| \leq 2 \Delta t \lambda_{1}^{-1}\left|\nabla \cdot \vec{U}_{p}\right|_{\infty}\left\|z_{p}^{n}\right\|\left\|z_{p}^{n+1}\right\|$ $\leq \frac{1}{2} \epsilon_{5} \nu_{0} \Delta t\left\|z_{p}^{n+1}\right\|^{2}+2 \epsilon_{5}^{-1} \nu_{0}^{-1} \lambda_{1}^{-2} \Delta t\left|\nabla \cdot \vec{U}_{p}\right|_{\infty}^{2}\left\|z_{p}^{n}\right\|^{2}$ b6) $-2 \Delta t\left(z_{p}^{n} \nabla \cdot \vec{U}_{q}, z_{p}^{n+1}\right) \leq 2 \Delta t\left|\nabla \cdot \vec{U}_{q}\right|_{\infty}\left|z_{p}^{n}\right|\left|z_{p}^{n+1}\right| \leq 2 \Delta t \lambda_{1}^{-1}\left|\nabla \cdot \vec{U}_{q}\right|_{\infty}\left\|z_{p}^{n}\right\|\left\|z_{p}^{n+1}\right\|$ $\leq \frac{1}{2} \epsilon_{5} \nu_{0} \Delta t\left\|z_{p}^{n+1}\right\|^{2}+2 \epsilon_{5}^{-1} \nu_{0}^{-1} \lambda_{1}^{-2} \Delta t\left|\nabla \cdot \vec{U}_{q}\right|_{\infty}^{2}\left\|z_{p}^{n}\right\|^{2}$ b7) $\begin{aligned}-2 \Delta t\left(\vec{U}_{p} \cdot \nabla z_{q}^{n+1}, z_{p}^{n+1}\right. & \left.-z_{p}^{n}\right) \leq 2 \Delta t c_{1} \lambda_{N / 2}^{1 / 4}\left|\vec{U}_{p}\right|^{1 / 2}\left\|\vec{U}_{p}\right\|^{1 / 2}\left\|z_{q}^{n+1}\right\|\left|z_{p}^{n+1}-z_{p}^{n}\right| \\ & \leq \frac{1}{2} \epsilon_{4}\left|z_{p}^{n+1}-z_{p}^{n}\right|^{2}+2 \epsilon_{4}^{-1} \Delta^{2} t c_{1}^{2} \lambda_{N / 2}^{1 / 2}\left|\vec{U}_{p}\right|\left\|\vec{U}_{p}\right\|\left\|z_{q}^{n+1}\right\|^{2}\end{aligned}$ b8) $-\Delta t\left(z_{p}^{n} \nabla \cdot \vec{U}_{p}, z_{p}^{n}\right) \leq \Delta t\left|\nabla \cdot \vec{U}_{p}\right|_{\infty}\left|z_{p}^{n}\right|^{2} \leq \Delta t \lambda_{1}^{-1}\left|\nabla \cdot \vec{U}_{p}\right|_{\infty}\left\|z_{p}^{n}\right\|^{2}$ b9) $-\Delta t\left(z_{p}^{n} \nabla \cdot \vec{U}_{q}, z_{p}^{n}\right) \leq \Delta t\left|\nabla \cdot \vec{U}_{q}\right|_{\infty}\left|z_{p}^{n}\right|^{2} \leq \Delta t \lambda_{1}^{-1}\left|\nabla \cdot \vec{U}_{q}\right|_{\infty}\left\|z_{p}^{n}\right\|^{2}$ b10) $-2 \Delta t\left(\vec{U}_{p} \cdot \nabla z_{p}^{n}, z_{p}^{n+1}-z_{p}^{n}\right) \leq 2 \Delta t c_{1} \lambda_{N / 2}^{1 / 4}\left|\vec{U}_{p}\right|^{1 / 2}\left\|\vec{U}_{p}\right\|^{1 / 2}\left\|z_{p}^{n}\right\|\left|z_{p}^{n+1}-z_{p}^{n}\right|$ $\leq \frac{1}{2} \epsilon_{4}\left|z_{p}^{n+1}-z_{p}^{n}\right|^{2}+2 \epsilon_{4}^{-1} \Delta^{2} t c_{1}^{2} \lambda_{N / 2}^{1 / 2}\left|\vec{U}_{p}\right|\left\|\vec{U}_{p}\right\|\left\|z_{p}^{n}\right\|^{2}$ 


$$
\begin{aligned}
& \text { b11) }-2 \Delta t\left(\vec{U}_{q} \cdot \nabla z_{p}^{n}, z_{p}^{n+1}-z_{p}^{n}\right) \leq 2 \Delta t c_{1} \lambda_{N / 2}^{1 / 4}\left|\vec{U}_{q}\right|^{1 / 2}\left\|\vec{U}_{q}\right\|^{1 / 2}\left\|z_{p}^{n}\right\|\left|z_{p}^{n+1}-z_{p}^{n}\right| \\
& \leq \frac{1}{2} \epsilon_{4}\left|z_{p}^{n+1}-z_{p}^{n}\right|^{2}+2 \epsilon_{4}^{-1} \Delta^{2} t c_{1}^{2} \lambda_{N / 2}^{1 / 2}\left|\vec{U}_{q}\right|\left\|\vec{U}_{q}\right\|\left\|z_{p}^{n}\right\|^{2} \\
& \text { b12) }-2 \Delta t\left(z_{q}^{n+1} \nabla \cdot \vec{U}_{p}, z_{p}^{n+1}\right) \leq-2 \Delta t\left|\nabla \cdot \vec{U}_{p}\right|_{\infty}\left|z_{q}^{n+1}\right|\left|z_{p+1}^{n}\right| \\
& \leq-2 \Delta t \lambda_{1}^{-1 / 2} \lambda_{N}^{-1 / 2}\left|\nabla \cdot \vec{U}_{p}\right|_{\infty}\left\|z_{q}^{n+1}\right\|\left\|z_{p+1}^{n}\right\| \\
& \leq \frac{1}{2} \epsilon_{5} \nu_{0} \Delta t\left\|z_{p}^{n+1}\right\|^{2}+2 \epsilon_{5}^{-1} \nu_{0}^{-1} \lambda_{1}^{-1} \lambda_{N}^{-1} \Delta t\left|\nabla \cdot \vec{U}_{p}\right|_{\infty}^{2}\left\|z_{q}^{n+1}\right\|^{2} \\
& \text { b13) }-2 g_{0} \Delta t\left(\vec{U}_{p} \cdot \nabla \vec{V}_{p}^{n}, \vec{V}_{p}^{n}\right) \leq g_{0} \Delta t \int_{D}\left(\nabla \cdot \vec{U}_{p}\right)\left(\vec{V}_{p}^{n} \cdot \vec{V}_{p}^{n}\right) \leq g_{0} \Delta t\left|\nabla \cdot \vec{U}_{p}\right|_{\infty} \lambda_{1}^{-1}\left\|\vec{V}_{p}^{n}\right\|^{2} \\
& \text { b14) }-2 g_{0} \Delta t\left(\vec{U}_{q} \cdot \nabla \vec{V}_{p}^{n}, \vec{V}_{p}^{n}\right) \leq g_{0} \Delta t \int_{D}\left(\nabla \cdot \vec{U}_{q}\right)\left(\vec{V}_{p}^{n} \cdot \vec{V}_{p}^{n}\right) \leq g_{0} \Delta t\left|\nabla \cdot \vec{U}_{q}\right|_{\infty} \lambda_{1}^{-1}\left\|\vec{V}_{p}^{n}\right\|^{2}
\end{aligned}
$$

Os termos contendo a forçante são estimados por:

$$
\text { b15) } \begin{aligned}
2 \Delta t\left(P_{N / 2} F, z_{p}^{n+1}\right) & \leq 2 \Delta t \lambda_{1}^{-1 / 2}\left|P_{N / 2} F\right|\left\|z_{p}^{n+1}\right\| \\
\leq & \frac{1}{2} \epsilon_{5} \nu_{0} \Delta t\left\|z_{p}^{n+1}\right\|^{2}+2 \epsilon_{5}^{-1} \nu_{0}^{-1} \lambda_{1}^{-1} \Delta t\left|P_{N / 2} F\right|^{2} \\
\text { b16) } 2 \Delta t\left(Q_{N} F, z_{q}^{n+1}\right) & \leq 2 \Delta t \lambda_{N / 2}^{-1 / 2}\left|Q_{N} F\right|\left\|z_{q}^{n+1}\right\| \\
\leq & \frac{1}{2} \epsilon_{6} \nu_{0} \Delta t\left\|z_{q}^{n+1}\right\|^{2}+2 \epsilon_{6}^{-1} \nu_{0}^{-1} \lambda_{N / 2}^{-1} \Delta t\left|Q_{N} F\right|^{2}
\end{aligned}
$$

Agora, sob a Hipotese (2.1), a condição (2.117) e escolhendo: $\epsilon_{1}=\frac{2}{3}, \epsilon_{3}=2, \epsilon_{4}=$ $\frac{2}{3}, \epsilon_{5}=\frac{1}{2}, \epsilon_{6}=2$, obtemos ao simplificar b1-b16 a estimativa (2.115).

As Proposições 2.2 e 2.3 indicam que para um número equivalente de modos o esquema de Galerkin não linear é mais estável que o de Galerkin Linear, com a diferença ficando no fator $\lambda_{N / 2}^{1 / 2}$ para Galerkin não linear e $\lambda_{N}^{1 / 2}$ para Galerkin linear. O mesmo tipo de relação nas restrições de estabilidade aparecem nas equações de Navier-Stokes (ver Teman [69] e J. Shen [61]). 


\section{Conclusões}

Nesta tese estudamos um modelo derivado das equações de água rasa no chamado plano-f, considerando a inclusão de termos viscosos, uma forçante na vertical e condições de contorno periódicas, sob um ponto de vista teórico pelas Variedades Inerciais Aproximadas, e numericamente, pelo método de Galerkin Não Linear.

Demonstramos a convergência das soluções do sistema associado às variedades inerciais aproximadas para as soluções do sistema original. As estimativas de erro obtidas foram da ordem de $\lambda_{m}^{-3 / 2}$ (onde $m$ corresponde ao número de modos usados pela projeção $P$, e $\lambda_{m}$ é o m-ésimo autovalor de $-\Delta$ ). Também foi demonstrada a convergência das soluções associadas ao método de Galerkin clássico para as soluções do sistema original, e o erro obtido foi da ordem de $\lambda_{m}^{-1}$. Observamos que para obter estas estimativas foi necessário assumir no caso geral a limitação das soluções das equações de água rasa (sob uma hipótese restritiva formulada por Aghoshov, estabelecemos a limitação das mesmas).

Propusemos um esquema numérico de Galerkin não linear para solução das equações de água rasa, o qual faz uso de um método pseudoespectral com discretização temporal de segunda ordem, com três níveis no tempo. Foi feita uma análise da eficiência computacional, obtendo ganhos de $12 \%$ com respeito ao método de Galerkin linear, e superior a $50 \%$ quando o congelamento dos modos altos é empregado. Observa-se que este ganho provem do tratamento dos termos não lineares, para um mesmo truncamento $N$ o método de Galerkin linear requer malhas mais finas que o método de Galerkin não linear para que os produtos fiquem livres de 'aliasing'. Quanto à precisão, os experimentos conduzidos aqui 
indicam que a precisão do método de Galerkin não linear com um determinado número de modos é quase tão boa quanto a do correspondente método de Galerkin linear, porém a um custo computacional menor. Esta precisão não sofre grande impacto se o congelamento dos modos altos é empregado (o que esta de acordo com a teoria das variedades inerciais aproximadas para $N$ suficientemente grande). Não foram observadas diferenças significativas quando a forma diagnóstica ou prognóstica dos modos altos são utilizadas. Para se aferir esta precisão dos métodos de Galerkin não linear e linear, foram conduzidos experimentos com a equação de Burgers uni-dimensional, caso em que soluções analíticas são conhecidas. Estes experimentos com a equação de Burgers indicam o mesmo comportamento observado nas equações de água rasa.

Foi estabelecida a estabilidade destes esquemas, linearizados em torno de um estado básico constante, e em torno de um estado básico com variação espacial (com o divergente do campo das velocidades suficientemente pequeno). As condições de estabilidade do método não linear são menos restritivas que as do método linear com o mesmo número de modos, mas experimentos numéricos indicam que o uso de passo maiores de tempo (permitidos pelas condições de estabilidade) para o método de Galerkin não linear pode levar a perda significativa na precisão.

No futuro, desejamos estender esta analise teórica das Variedades Inerciais Aproximadas e os métodos de Galerkin não linear para as equações de água rasa na esfera. Isto é possível pois pode-se usar transformadas de Fourier na longitude, e polinômios de Legendre na latitude. Para isto temos que obter uma implementação eficiente para os polinômios de Legendre (Shen [62], [63] tem estudos prévios para métodos de Galerkin não linear sobre estes polinômios) os quais devem ser combinados com as transformadas de Fourier. 


\section{Referências Bibliográficas}

[1] V.I. Agoshkov, D. Ambrosi, V. Pennati, A. Quarteroni and F. Saleri, Mathematical and numerical modelling of shallow water flow, Computational Mechanics (1993) 11, 280-299.

[2] V.I. Agoshkov, E. Ovchinnikov, A. Quarteroni and F. Saleri, Recent developments in the numerical simulation of shallow water equations. II. Temporal discretization, Mathematical Models and Methods in Appllied Sciences, Vol 4, No. 4 (1994), 533-556.

[3] S.R.M. Barros, Métodos Espectrais para modelos meteorológicos. Uma Intro$d u c ̧ a \tilde{o}, \quad$ Notas do minicurso apresentado no Workshop Computational Methods for Oceanic and Atmospheric Flows, LNCC, RJ, 1995.

[4] J. Boyd, Time-Marching on the Slow Manifold: The relationship between the Nonlinear Galerkin Method and Implicit Timestepping Algoritms, Appl. Math. Lett., Vol. 7, No. 2, pp. 95-99, 1994.

[5] C. Canuto, Y. Husaini, A. Quarteroni and T. Zang, Spectral Methods in Fluid Dynamics, Springer Verlag, New York, 1988.

[6] J.W. Cárdenas, Técnicas Analiticas e Computacionais para um Modelo Atmosférico de Lorenz, Dissertação de Mestrado. Universidade Federal do Rio grande do Sul, Brasil, 1994.

[7] J.W. Cárdenas, M. Thompson, Inertial Approximate Manifolds for Lorenz Models, $42^{\circ}$ Seminario Brasileiro de Análise, 1995. 
[8] J.W. Cárdenas, Nonlinear Galerkin Methods for Burgers Equation 1D, Em preparação.

[9] P. Christofides, Finite-Dimensional Control of Parabolic PDE System using AIM, Journal of Math. Anal. and Applications, Vol. 216, No. 2, December 15, 1997, pp. 389-420.

[10] J. Cole, On a quasilinear parabolic equation ocurring in aerodynamics, Q. Appl. Math. 9, pp. 225-236, 1951.

[11] P. Constantin, C. Foias, Navier Stokes Equations, Chicago Lectures in Mathematics Series, 1988.

[12] J. Cooley and J. Tukey, An alghorithm for the Machine Calculation of Complex Fourir Series, Math. comp. 19, 1965.

[13] J.H. Curry, D. Winsand, Low Order Intermediate Models: Bifurcation, Recurrence and Stability, Journal Atmospheric Sciences,43, 2360-2373, 1986.

[14] S. Ghildress, M. Ghil, Topics in Geophysical Fluid Dynamics; Atmospheric Dynamics, Dynamo Theory and Climate Dynamics, Springer-Verlag, Berlin, 1987.

[15] R. Daley, The develpoment of efficient time integration schemes using model normal modes, Mon. Weather Rev., 108, pp. 100-110, 1980.

[16] A. Debussche and M. Marion, On the construction of families of approximate inertial manifolds, J. Differential Equations 100, pp. 173-201, 1992.

[17] A. Debussche, T. Dubois, R. Temam, The nonlinear Galerkin method: A multi-scale method applied to the simulation of homogeneos turbulent flows, Icase Report No. 93-93. December 1993.

[18] A. Debussche and T. Dubois, Convergent families of approximate inertial manifolds, T. 73, N0. 5, 1994, pp. 489-521.

[19] L. Dettori, D. Gottlieb and R. Temam, A Nonlinear Galerkin Method: The Two-Level Fourier-Collocation Case, Journal of Scientific Computing, Vol. 10, No. 4, 1995. 
[20] C. Devulder, M. Marion, E.S. Titi, On the Rate of Convergence of the Nonlinear Galerkin Methods, Math. Comput; 60, 1993, 495-514.

[21] T. Dubois, F. Jabertau et R. Temam, Solution of the incompressible Navier-Stokes equations by the Nonlinear Galerkin Methods, J. Sci. Comput. Vol 8(2), 1993, pp. 167-194.

[22] T. Elvius, A. Sundström, Computationally efficient schemes and boundary conditions for a fine-mesh barotropic model based on the shallow-water, Tellus XXV, 2, 1973, pp. $132-156$.

[23] C. Foias, O.P. Manley, R. Temam, Modelization of the iteration of small and large eddies in two dimensional turbulent flows, Math. Model. Numer. Anal. $M^{2} N A, 22$, 1988, 93-114.

[24] C. Foias, O.P. Manley, R. Temam, Approximate Inertial Manifold and Viscosity in Turbulent Flows, $\quad$ Phys.Fluids A3, 5, 1991 , 898-911.

[25] C. Foias, G. Shell, R. Teman, Inertial manifolds for nonlinear evolutionary equations, J. Differential Equations, 73, 1988, 309-353.

[26] C. Foias, G. Shell, E.S. Titi, Exponential tracking and approximation of inertial manifolds for dissipative nonlinear equations, J. Dynam. Diff.Eq., 1 (2), 1989, 199243.

[27] D. Gottileb and R. Temam, Implementation of the Nonlinear Galerkin Method with pseudospectral (collocation) discretizations, Appl. Num. Math. 12, 1993, pp. 119-134.

[28] O. Goubet, Construction of Approximate Inertial Manifolds using Wavelets, SIAM J. Math. Anal., Vol. 23, No. 6, pp. 1455-1481, November 1992.

[29] M. Graham, P. Steen and E. Titi, Dynamics of Bénard convection in porous media: computations with approximate inertial manifolds, J. Nonlinear Sci. 3, pp. 153167, 1993. 
[30] G. Haltiner and R. Williams, Numerical Prediction and Dynamic Metereology, Ed. John Wiley \& Sons, Inc., 1980.

[31] J.G. Heywood, R. Rannacher, Finite Element Approximation of the Nonstationary Navier-Stokes Problem, I, SIAM J.Numer.Anal; 19, 1982, 275-311.

[32] J.G. Heywood, R.Rannacher, On the Question of Turbulence Modeling by Approximate Inertial Manifolds and the Nonlinear Galerkin Method, SIAM J.Numer.Anal; 30. 1993, 1603-1621.

[33] F. Jabertau, C. Rosier and R. Temam, The Nonlinear Galerkin Method in Computational Fluid Dynamics, Applied Numerical Mathematics; 6, 1989/90, p.361-370.

[34] F. Jabertau, C. Rosier and R. Temam, A Nonlinear Galerkin Method for the NavierStokes Equation, Computer Meth. in Appl. Mechanics and Engineering, Vol. 80. p.245, 1990.

[35] M. Jarraud and A. Baede, The use of Spectral Techniques in Numerial Weather Prediction, Lectures in Applied Mathematics, Vol. 22, 1985.

[36] M. Jolly, I. Kevrekidis, E. Titi, Approximate inertial manifolds for the KuramotoSivashinski equation: analysis and computations, Phys. D, 44, 1990, 38-60.

[37] M. Jolly, Bifucartion computations on an approximate inertial manifolds for the Navier-Stokes equations, Phys. D 63, 8-20, 1993.

[38] M.S. Jolly and C. Xiong, On Computing the Long-Time Solution of the TwoDimensional Navier-Stokes Equations, Theoret. Comput. Fluid Dynamics, 7, pp. 261-27S, 1995.

[39] D.A. Jones, E.S. Titi, A remark on quasi-stationary approximate inertial manifolds for the 2D Navier-Stokes equations, SIAM J. Math. Anal., 25, 1994, 894-914.

[40] D.A. Jones, L.G. Margolin, E.S. Titi, On the Effectiveness of the Approximate Inertial Manifold - a Computational Study, Theoret. Comput. Fluid Dynamics; 7, 1995. $243-260$. 
[41] N. Kopell, Invariant Manifolds and the Initialization Problem for Some Atmospheric Equations, Physica D14 1985, 203-215.

[42] H. Kreiss and O. Oliger, Stability of te Fourier Methods, SIAM J. Numer. Anal. 16, pp. 421-433, 1979.

[43] J. Laminie, F. Pascal, R. Temam, Implemantation of finite element nonlinear methods using hierarchical bases, Computational Mechanics, 1993, 11, 384-407.

[44] B. Legras, R. Vautard, Invariant Manifolds, Quasi-Geostrophy and initialization, Journal Atmospheric Sciences, 43, 1986, 565-584.

[45] X. Liu, On Approximate Inertial Manifolds for Two and Three Dimensional Turbulent Flows, J.Math, Anal.Appl, 163, 1992, 559-580.

[46] E. Lorenz, Attractor Sets and Quasi-Geostrophic Equilibrium, J.Atmospheric Sciences, 37,1980, 1685-1689.

[47] E. Lorenz, On the Existence of a Slow Manifold, J.Atmospheric Sciences, 43, 1986, $1547-1557$.

[48] M. Marion and R. Temam, Nonlinear Galerkin Methods, SIAM J. Num. Anal., Vol 26, pp. 1139-1157, 1989.

[49] M. Marion, Approximate inertial manifolds for the pattern formation Cahn-Hilliard equations, Math. Model. Numer. Anal. 23, pp. 463-488, 1989.

[50] M. Marion, Approximate inertial manifolds for reaction-diffusion equations in high space dimension, J. Dinamics Differential Equations, 1, pp . 245-267, 1989.

[51] M. Marion and R. Temam, Nonlinear Galerkin Methods: the finite elements case, Numer. Math., 57, pp. 205-226, 1990.

[52] T. Matsuno, Quasi-Geostrophic Motions in the Equatorial Area, Journal of Meterological Society of Japan, Vol. 44, No. 1, February 1966.

[53] R. Meyer, Introduction to Mathematical Fluid Dynamics, Pure and Applied Mathematics, Vol. XXIV, 1971. 
[54] S. Orszag, Numerical Simulation of Incompressible Flows Within Simple boundaries. I. Galerkin (Spectral) Representations, Studies in Applied Mathematics, Vol. L, No. 4, December 1971.

[55] S. Orszag, in Proc. $5^{\text {th }}$ Intl. Conf. on Numerical Methods in Fluid dynamics, Lectures Notes in Physics, Vol. 59, p. 32, 1977.

[56] S.Panchev, Dynamic Meteorology, D.Reidel Publishing Co; Dordretch, 1985.

[57] Pedoskly, J, Geophysical Fluid Dynamics, Springer, N.Y. 1979.

[58] K. Promislow, Induced trajectories and approximate inertial manifolds for the Ginzburg-Landau PDE, Phys. D 41, pp. 232-252, 1990.

[59] R. Russell, D. Sloan and M. Trummer, Some numerical aspects of computing inertial manifolds, SIAM J. Sci. Comput., 14, pp. 19-43, 1993.

[60] M.A. Raupp, Dinâmica não linear e Métodos de Galerkin, $39^{0}$ Seminario de Análise. 1994.

[61] J. Shen, Long time stability and convergence for fully discrete nonlinear Galerkin methods., Applied Anal., 38 (1990), pp. 201-229.

[62] J. Shen, R. Temam, Nonlinear Galerkin Method using Legendre Polynomials., Proceedings of the International Conference on Nonlinear Evolution Partial Differential Equation., June 1993, Beijin, China.

[63] J. Shen, R. Temam, Nonlinear Galerkin Method using Chebyshev and Legendre Polynomials I. The one dimensional case, Siam J. Numer Anal., Vol 23, No 1, pp. 215-234, February 1995.

[64] R. Temam, Navier Stokes Equations North-Holland, Amsterdam, 1977.

[65] R. Temam, Navier-Stokes Equations and Nonlinear Functional Analysis, SIAM, 1983.

[66] R. Temam, Infinite Dimensional Dynamical Systems in Mechanics and Physics, Springer-Verlag, Berlin, 1988. 
[67] R. Temam, Induced trajectories and approximate inertial manifolds, ( $\left.\mathrm{M}^{2} \mathrm{AN}\right), \mathrm{Vol}$. 32 , p. $163,1988$.

[68] R. Temam, Inertial Manifolds and Multigrids methods, SIAM, J. Math. Anal., vol. 21, 1990, pp. 154-178.

[69] R. Temam, Stability analysis of the nonlinear Galerkin method, Math. Comp., 57 (1991), pp. 477-505.

[70] E. Titi, On Approximate Inertial Manifolds to the Navier-Stokes Equations, J.Math. Anal.Appl, 149, 1990, 540-557.

[71] B. Wang, Attractors and Approximate Inertial Manifolds for the Generalized Benjamin-Bona-Mahony Equation, Mathematical Methods in the Applied Sciences, Vol. 20, pp. 189-203, 1997. 